\title{
Sparse Recovery, Classification, and Data Compression via \\ Improved Maximum Feasible Subsystem Algorithms
}

\author{
by \\ Fereshteh Fakhar Firouzeh
}

A thesis submitted to the Faculty of Graduate Studies and Research in partial fulfillment of the requirements for the degree of

Doctor of Philosophy

in

Electrical and Computer Engineering

Carleton University

Ottawa, Ontario

(C) 2021, Fereshteh Fakhar Firouzeh 


\section{Abstract}

Two new strategies are developed in this thesis to increase the speed of the state-ofthe-art Maximum Feasible Subsystem (MAX FS) algorithms. The newly developed strategies can be combined with any MAX FS algorithm to increase its speed while preserving or improving solution quality. The improved algorithms apply in the case of dense constraint matrices such as those found when various data compression/dimensionality reduction, classification, and sparse recovery problems are converted to MAX FS problems. This approach is used for sparse recovery in Compressive Sensing (CS) and data compression via Nonnegative Matrix Factorization NNMF for the first time in this thesis. In CS, the new algorithm can successfully recover real-world signals such as speech and Electrocardiogram (ECG) signals that have been more greatly compressed than existing methods can handle, and with greater recovered signal quality. They also deliver sparser solutions when compared with those obtained using traditional sparse recovery algorithms. The new algorithm significantly reduces the solution time of the existing MAX FS methods. It reduces the solution time of Method B by $80.64 \%$ and $77.82 \%$ for recovery of compressively sensed ECG and speech signals for instance, respectively. Three novel MAX FS-based methods for NNMF are developed and investigated using synthetic data and real-world image datasets. The new NNMF algorithms, unlike the majority of existing NNMF methods, do not require an initialization method or prior knowledge of the matrix rank. 
These new NNMF solutions methods are also more robust to outliers and provide higher quality solution compared to the state-of-the-art NNMF algorithms. To illustrate, the new NNMF algorithm improves the relative approximation quality of the existing column subset selection NNMF algorithm by $5.2 \%$ for the ORL facial image dataset. A faster MAX FS-based algorithm for binary classification is also proposed, which improves the processing time of the existing MAX FS algorithm (Algorithm 2) by $94.77 \%$ on average. In comparison, the developed algorithm outperforms widely used binary classification algorithms by providing better results for recall-oriented machine learning tasks such as disease diagnosis. For instance, the new algorithm improves the average recall score of Support Vector Machine (SVM) by $18.16 \%$ for binary classification task. 


\section{Acknowledgements}

My PhD adventure transformed my life and broadened my perspective! One thing I learnt and felt the most is the great impact of incredible people around me in being who I am today. I am so grateful for having this opportunity to express my gratitude to some of those wonderful people, who provided their unconditional support during my $\mathrm{PhD}$.

First and foremost, I would like to express my sincere appreciation to Professor John W. Chinneck and Professor Sreeraman Rajan for giving me the opportunity to do my research under their supervision. I am grateful for their invaluable guidance and patience throughout my PhD degree. Words fail me to describe how grateful I am for having the privilege to work with them and learn from their immense knowledge. Thank you for all you have done for me.

I would like to express my appreciation to my advisory committee consisting of Professor Richard Dansereau, Professor Hilmi Dajani, Professor John Oommen, Professor Sridhar Krishnan, and Professor Martin Bouchard. I will forever be thankful to

Professor Richard Dansereau for his support and insightful comments, which assisted me to achieve my goals.

I am especially grateful to my colleagues, Mohamed Abdelazez and Dipayan Mitra, for always willing to share their experience and for their valuable support. 
I would also like to acknowledge Carleton University and Natural Sciences and Engineering Research Council of Canada (NSERC) for the financial support, which provided me the opportunity to focus on my research.

I will forever be thankful to my master's superviso, Dr. Alireza Ghorshi, who literally changed and still is changing my life. He is a great mentor and always inspires me to be the best version of myself. I feel extremely lucky and privileged to know someone as thoughtful and as kind as you.

There are no words that can express my gratitude towards my father and mother who always motivated me and supported me towards my goals. I am so thankful for you in my life. I appreciate and value all that you have sacrificed. Thanks to my wonderful sisters, brothers, and my beautiful nieces and nephews; you are the concretely irreplaceable part of my life and I love you all. My younger brother, Mohammad Hassan, has always been one true inspiration. Thank you for being the one-of-a-kind special someone in my life. I would also thank my besties, Paria Samimi and Molood Jahanyar, for always being there for me. I am grateful to you for being true friends through my life journey.

Last but not least, I would like to thank my husband, Dr. Sina Salsabili, for all the support during these years. Since the first day I met you, you have been the miracle that makes my life complete. I cannot imagine where I would be without your love, guidance, and care. Thank you, my love, for walking right into the storm with me and being a comfort when I needed it the most. Having you in my life is such a precious gift! 


\section{Contents}

$\begin{array}{ll}\text { Abstract ii } & \text { ii }\end{array}$

Acknowledgements iv

List of Tables $\quad$ ix

List of Figures $\quad$ xiii

\begin{tabular}{|ll}
\hline Acronyms & xvi
\end{tabular}

Nomenclature xxiii

$\begin{array}{lll}1 & \text { Thesis in a Nutshell } & 1\end{array}$

1.1 Overview of Contributions . . . . . . . . . . . . . . . . . . . 1

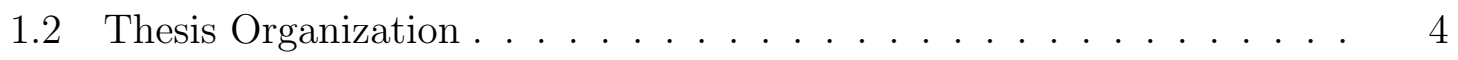

1.3 Publications . . . . . . . . . . . . . . . . . 5

$\begin{array}{|lll|}2 & \text { The Maximum Feasible Subsystem Problem (MAX FS) } & 7\end{array}$

$2.1 \quad$ Existing MAX FS Methods for Sets of Linear Constraints . . . . . . . 8

2.1 .1 Provably Optimal Formulations . . . . . . . . . . . . . . 8

$2.1 .2 \quad$ Irreducible Infeasible Sets (IIS) Covering . . . . . . . . . . . . 10

2.1 .3 Phase one Heuristics . . . . . . . . . . . . . . . . . 11 
2.1 .4 Chinneck's MAX FS Algorithms . . . . . . . . . . . . . . 12

2.1 .5 Two Phase Heuristics. . . . . . . . . . . . . . . . . . . 20

$2.1 .6 \quad$ Randomized Thermal relaxation Algorithms . . . . . . . . 22

2.1 .7 Linear Programming Interior Point-based heuristics . . . . . 23

2.2 Discussion and Comparison $\ldots \ldots \ldots \ldots \ldots \ldots$

\begin{tabular}{lll}
\hline Problem Statement & 25
\end{tabular}

3.1 Deficiencies of the State-of-the-Art $\ldots \ldots \ldots \ldots \ldots$

\begin{tabular}{|lll}
4 & New MAX FS Algorithms for Dense Constraint Matrices & 28
\end{tabular}

4.1 Extensions for Dense Constraint Matrices. . . . . . . . . . . . . . . 29

$4.1 .1 \quad$ Extension 1 (E1): Multiple Simultaneous Removals . . . . . . 31

4.1 .2 Extension 2 (E2): Early Exit $\ldots \ldots \ldots \ldots \ldots$

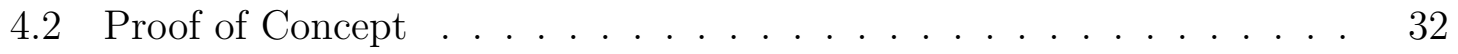

$4.2 .1 \quad$ Experimental Setup . . . . . . . . . . . . . . . . . . 33

4.2 .2 Results and Discussion . . . . . . . . . . . . . . . 35

5 Extended MAX FS for Binary Classification 39

$5.1 \quad$ Existing Binary Classification Algorithms. . . . . . . . . . . . . 41

5.2 Extended MAX FS Binary Classification Algorithm . . . . . . . . . 46

5.3 Experimental Setup . . . . . . . . . . . . . . . . . . . . . . 48

5.4 Results and Discussion . . . . . . . . . . . . . . . . . . 52

$\begin{array}{lll}6 & \text { Extended MAX FS for Sparse Recovery in Compressive Sensing } & 59\end{array}$

$6.1 \quad$ Compressive Sensing (CS) $\ldots \ldots \ldots \ldots \ldots$

$6.2 \quad$ Existing Algorithms for CS Sparse Recovery . . . . . . . . . . . . 68

6.3 New Extended MAX FS Algorithm for CS Recovery. . . . . . . . . . 86

$6.4 \quad$ MAX FS for Sparse Recovery of Compressively Sensed ECG Signals . 87 
$6.4 .1 \quad$ Experimental Setup . . . . . . . . . . . . . . . . . 90

6.4 .2 Results and Discussion . . . . . . . . . . . . . . . . . . . . . . 92

6.5 MAX FS for Sparse Recovery of Compressively Sensed Speech Signals 102

6.5 .1 Experimental Setup . . . . . . . . . . . . . . . 106

6.5 .2 Results and Analysis . . . . . . . . . . . . . . . . . . . . 108

7 New MAX FS Algorithm for Non-Negative Matrix Factorization (NNMF) 121

7.1 Non-Negative Matrix Factorization (NNMF) $\ldots \ldots \ldots \ldots \ldots$

7.2 Existing Algorithms for the NNMF Column Selection Problem . . . . 125

7.3 New NNMF Algorithms via MAX FS . . . . . . . . . . . . . . . 131

$7.3 .1 \quad$ Feasible Subset Matrix Factorization (FSMF) $\ldots \ldots \ldots$. . . 132

7.3.2 $\quad$ Fast Feasible Subset Matrix Factorization Algorithm (FFSMF) 133

7.3.3 $\quad$ Feasible Subset Matrix Factorization - Partitioned (FSMF-P) 135

7.4 Experimental Setup . . . . . . . . . . . . . . . . . . 137

7.5 Results and Discussion . . . . . . . . . . . . . . . . . 142

8 Conclusions and Future Work 149

8.1 Conclusions . . . . . . . . . . . . . . . . . . . . . . . . . 149

$8.2 \quad$ Summary of Contributions $\ldots \ldots \ldots \ldots \ldots \ldots$

8.3 Future Research . . . . . . . . . . . . . . . . . . . . . . . . 152

\begin{tabular}{ll}
\hline References & 154
\end{tabular} 


\section{List of Tables}

2.1 Constraint elasticizing by using non-negative elastic variables. . . . . 12

4.1 Comparing the performance of Method B, Algorithm BE1, Algorithm $\mathrm{B}(2) \mathrm{E} 2(\ell)$, and Algorithm BE1E2( $\ell)$ using the average sparsity error (\%) in sparse recovery $(m=128, n=256$, and results are averaged over 100 random matrices). . . . . . . . . . . . . . . . . . . . 36

4.2 Number of LPs and processing times for Algorithm BE1, Algorithm $\mathrm{B}(2) \mathrm{E} 2(\ell)$, and Algorithm BE1E2( $\ell)$ vs. Method B(2) in sparse recovery $(m=128, n=256$, and results are averaged over 100 random matrices). . . . . . . . . . . . . . . . . . . . 37

4.3 Differences from the smallest LPs and processing time (the results averaged over 100 random matrices). . . . . . . . . . . . . . . . . . . . 38

5.1 Configuration space for the hyperparameters and methods of the comparators. . . . . . . . . . . . . . . . . . . . 49

5.2 Classification Datasets for Scenario I. . . . . . . . . . . . . . . . . . 51

5.3 Classification Datasets Scenario II. . . . . . . . . . . . . . . . . . . . 51

5.4 Algorithm Accuracies for Classification Datasets. . . . . . . . . . . 53

5.5 Differences from Best Accuracy. . . . . . . . . . . . . . . . . . . . 53

5.6 Algorithm speeds (number of LPs, seconds) for binary classification. . 54 
5.7 Percentage Reductions in LPs Solved and Solution Times by Using Algorithm 2E1 instead of original Algorithms 2( $\infty)$ and 2(1) for Classification. . . . . . . . . . . . . . . . . . 55

5.8 Performance of binary classification models in terms of Precision, Recall, F1 score, accuracy, and processing time (10-fold cross validation). 56

$5.9 \quad$ Differences from the best results. (10-fold cross validation). . . . . . . 57

6.1 Comparing three different categories of the sparse estimation algorithms. 82

6.2 Complexity and minimum number of measurements of some state of the art sparse estimation algorithms. . . . . . . . . . . . . . . . . 82

6.3 Comparative summary of CS recovery algorithms. . . . . . . . . . . . 83

6.4 Comparision of the performances of Method B, Method M, and Algorithm ME1E2( $\ell)$ using the average sparsity error (\%) in estimating the sparsity level of 100 ECG segments of length $n=360$ at $C R=50 \%$. . 96

6.5 Comparison of performances of the conventional algorithms using the average sparsity error (\%) in estimating the sparsity level of 100 ECG segments of length $n=360$ at $C R=50 \%$. . . . . . . . . . . . . 97

6.6 Comparing average number of LPs and processing time in seconds of Method B, Method M, and the proposed Algorithm ME1E2( $\ell$ ) for 100 ECG segments of length $n=360$ at $C R=50 \%$. . . . . . . . . . . . 97

6.7 Average percentage reductions in LPs solved and processing time by \begin{tabular}{|c|}
\hline using Algorithm ME1E2 $(\ell)$ instead of Method B and Method M for \\
\hline
\end{tabular} 100 ECG segments of length $n=360$ at $C R=50 \%$. . . . . . . . . . 98

6.8 Comparing average processing time in seconds of the conventional algo-

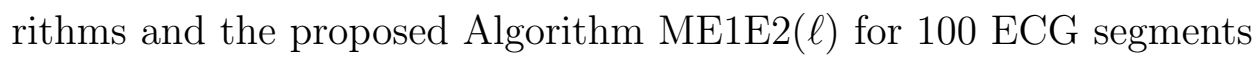
of length $n=360$ at $C R=50 \%$. . . . . . . . . . . . . . . . . . . . . 98 
6.9 Average algorithm speeds (number of LPs, Time in seconds) averaged over 47 ECG signals of length 3600 at different $C R$ s. . . . . . . . . . 100

6.10 Average percent reduction in number of LPs and processing times for Algorithm ME1E2( $\ell$ ) vs. Methods B, and M for 47 ECG signals of

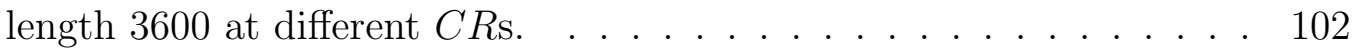

6.11 Average processing time (secs) required by Algorithm ME1E2 $\ell$ and conventional sparse recovery methods for 47 ECG signals of length

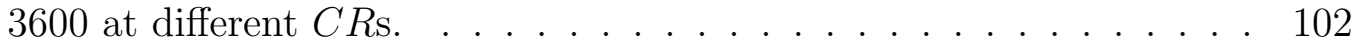

6.12 Comparing performance of different sparse recovery algorithms using the average sparsity error (\%) in estimating the sparsity level of 100 speech LFR segments of length $n=256$ at $C R=50 \%$. . . . . . . . 111

6.13 Comparing performance of different sparse recovery algorithms using

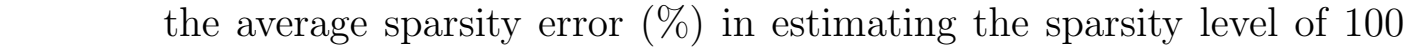
speech HFR segments of length $n=256$ at $C R=50 \%$. . . . . . . . . 112

6.14 Comparing average number of LPs and processing time in seconds of Method B, Method M, and Algorithm ME1E2( $\ell$ ) for 100 speech LFR segments of length $n=256$ at $C R=50 \%$. . . . . . . . . . . . . 112

6.15 Comparing average number of LPs and processing time in seconds of Method B, Method M, and the proposed Algorithm ME1E2( $\ell$ ) for 100 speech HFR segments of length $n=256$ at $C R=50 \%$. . . . . . . . . 113

6.16 Average percentage reductions in LPs solved and processing time by using Algorithm ME1E2 $(\ell)$ instead of Method B and Method M for 100 speech LFR segments of length $n=256$ at $C R=50 \%$. . . . . . 113

6.17 Average percentage reductions in LPs solved and processing time by using Algorithm ME1E2 $(\ell)$ instead of Method B and Method M for 100 speech HFR segments of length $n=256$ at $C R=50 \%$. . . . . . . 114 
6.18 Comparing average processing time in seconds of the conventional algorithms and the proposed Algorithm ME1E2( $\ell$ ) for 100 LFR speech segments of length $n=256$ at $C R=50 \%$. . . . . . . . . . . . . . . . 114

6.19 Comparing average processing time in seconds of the conventional algorithms and the proposed Algorithm ME1E2( $\ell)$ for $100 \mathrm{HFR}$ speech segments of length $n=256$ at $C R=50 \%$. . . . . . . . . . . . . . . 115

6.20 Comparing formants and sparsity level of the female original signal with the recovered signal using RNM. . . . . . . . . . . . . . . . . . . 120

6.21 Comparing formants and sparsity level of the male original signal with the recovered signal using RNM. . . . . . . . . . . . . . . . . . . . . . 120

7.1 The relative approximation quality in percent for the facial image data sets. . . . . . . . . . . . . . . . . . . . . 148 


\section{List of Figures}

$2.1 \quad$ Different categories of the existing MAX FS methods. . . . . . . . . . 9

2.2 Algorithm 1 . . . . . . . . . . . . . . . . . . . . . . . 14

2.3 Method B . . . . . . . . . . . . . . . . . . . . . . . . . . . 19

2.4 Method C . . . . . . . . . . . . . . . . . . 21

4.1 Algorithm BE1 . . . . . . . . . . . . . . . . . . . . . . . . . . . . . . 32

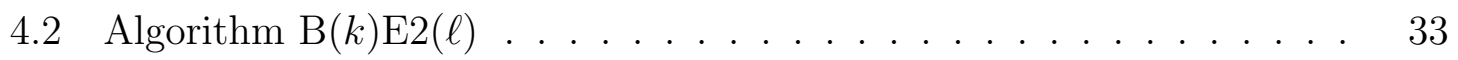

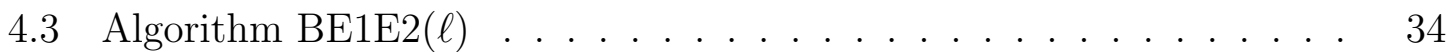

5.1 Algorithm 2E1 . . . . . . . . . . . . . . . . . . . 47

5.2 First sorted candidate list for "Ozone Level Detection" dataset. . . . . . . . 47

5.3 Scenario II ROC_AUC Scores. . . . . . . . . . . . . . . . . . . 58

6.1 Different classes of measurement matrix $\mathbf{\Phi}$. . . . . . . . . . . . . . . . 65

6.2 Classification of the existing sparse recovery algorithms. . . . . . . . . 68

6.3 Procedure of greedy algorithms. . . . . . . . . . . . . . . . . 76

6.4 Orthogonal Matching Pursuit (OMP) . . . . . . . . . . . . . . . 79

6.5 Polytope Faces Pursuit $(\mathrm{PFP})$. . . . . . . . . . . . . . . . . 80

6.6 Multipath Matching Pursuit (MMP) . . . . . . . . . . . . . . . . 84 
6.7 Pipeline for compressing and recovering a compressively sensed ECG signal. . . . . . . . . . . . . . . . . . . . . . . . . . . . . . 89

6.8 The waveform of ECG signal 104m and one segment of length 360. . . . . 94

6.9 Comparing quality of the signal recovered by Method B and Algorithm ME1E2 $(\ell)$ using PRD, averaged over 47 ECG signals of length 3600 segmented into 10 frames of length $n=360$ at different $C R$ s. . . . . . . . . . 100

6.10 Comparing the input ECG signal and the signals recovered via the sparse recovery methods. The signals recovered by Methods B, M, ME1E2( $\ell)$, BP, MP, OMP, PFP, and SL0 have correlation coefficients of $0.983,0.983,0.997,0.986,0.954,0.986$, and 0.994 with the original

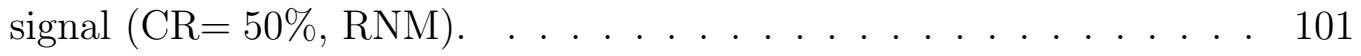

6.11 Pipeline for compressing and recovering a compressively sensed speech signal. . . . . . . . . . . . . . . . . . . . . 105

6.12 Example LFR and HFR segments of speech signal. . . . . . . . . . . . . 109

6.13 Average RSEs of 96 recovered signals. . . . . . . . . . . . . . . . . . . 116

6.14 Comparing average PESQ for 48 female and 48 male recovered speech signals using RNM. . . . . . . . . . . . . . . . . . . . . . . . . . . . 117

6.15 Comparing spectrogram of input female speech signal $F G J D 0_{S} X 9$ and reconstructed signals $(\mathrm{CR}=50 \%, \mathrm{RNM})$.

6.16 Comparing spectrogram of input male speech signal $M A E O 0_{S} A 2$ and reconstructed signals $(\mathrm{CR}=50 \%, \mathrm{RNM}) . \ldots$. . . . . . . . . . . . 119

$7.1 \quad$ Successive Projection Algorithm (SPA) . . . . . . . . . . . . . . . . . 126

7.2 Minimum-Volume NMF (MIN-VOL NMF) algorithm . . . . . . . . . 128

7.3 Accelerate Hierarchical Alternating Least Squares (A-HALS) . . . . . 129

7.4 Generalized Separable NMF with a Fast Gradient Method (GS-FGM) 129 
7.5 FSMF algorithm $\ldots \ldots \ldots \ldots$

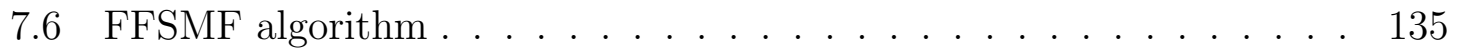

7.7 FSMF-P algorithm $\ldots \ldots \ldots \ldots \ldots$. . . . . . . . . . . . 136

7.8 Comparing the average root mean square residual of the proposed algorithms with MIN-VOL NMF, A-HALS, HALS, and MU over 100 trials $(m=50, n=200$ and $P=10)$. . . . . . . . . . . . . . . . . . 143

7.9 Comparing the average root mean square residual of the proposed algorithms with RSPA and SPA over 100 trials $(m=50, n=200$ and $P=10)$. 143

7.10 Percentage of recovered columns of $\mathbf{W}$ for the synthetic datasets using SPA, RSPA and NNMF MAX FS-based method. . . . . . . . . . . . . . . . . 144

7.11 (a) 6 images from Yale dataset, (b) approximation by GS-FGM, (c) approximation by FSMF-P algorithm. . . . . . . . . . . . . . . . . . . 145

7.12 (a) 6 images from CBCL dataset, (b) approximation by GS-FGM, (c) approximation by FSMF-P algorithm. . . . . . . . . . . . . . . . . . 146

7.13 (a) 6 images from ORL dataset, (b) approximation by GS-FGM, (c) approximation by FSMF-P algorithm. . . . . . . . . . . . . . . . . . . 147 


\section{Acronyms}

A-HALS Accelerated Hierarchical Alternating Least Squares. 126

AHA American Heart Association. 87

AMP Approximate Message Passing. 70

AUC Area Under the Curve. 50

AZTEC Amplitude Zone-Time-Epoch-Coding. 87

BAOMP Back-tracking based Adaptive Orthogonal Matching Pursuit. 69

BCS Bayesian compressive Learning. 69

BCW Breast Cancer Wisconsin. 51

BLD BUPA Liver Disorders. 51

BP Basis Pursuit. 20

BPDN Basis Pursuit De-noising. 70

BSS Blind Source Separation. 85

CBCL Centre for Biological and Computational Learning. 140

CORTES Coordinated Reduction-Time-Encoding System. 87 
CoSaMP Compressive Sampling Matching Pursuit. 76

CP Chaining Pursuit. 69

CR Compression Ratio. 65

CS Compressive Sensing. [i]

CVDs Cardiovascular diseases. 87

D-OMP Differential Orthogonal Matching Pursuit. 69

DCT Discrete Cosine Transform. 63

DRD Diabetic Retinopathy Debrecen. 51

DS Dantzig Selector. 70

DWT Discrete Wavelet Transform. 63

ECG Electrocardiogram. 25

FBP Forward-Backward Pursuit. 69

FFSMF Fast Feasible Subset Matrix Factorization Algorithm. 133

FISTA Fast Iterative Shrinkage Thresholding Algorithm. 69

FN False Negatives. 50

FOCUSS FOCal Underdetermined System Solution. 73

FP False Positive. 50

FSMF Feasible Subset Matrix Factorization. 132 
FSMF-P Feasible Subset Matrix Factorization - Partitioned. 135

GBP Greedy Basis Pursuit. 70

GNC Graduated Non-Convexity. 73

GOAMP Generalized Orthogonal Adaptive Matching Pursuit. 77

GOMP Generalized Orthogonal Matching Pursuit. 24

GP Gradient Pursuit. 76

GraDes Gradient Descent with Sparsification. 70

GS-FGM Generalized Separable NMF with a Fast Gradient Method. 126

HALS Hierarchical Alternating Least Squares. 128

HD Heart Disease. 51

HFR High Frequency Region. 108

HHS Heavy Hitters on Steroids. 69

ICA Independent Component Analysis. 122

IDC International Data Corporation. 39

IHT Iterative Hard Thresholding. 76

IIS Irreducible Infeasible Set. 10

IRWLS Iterative Re-weighted Least Squares. 74

IST Iterative Soft Thresholding. 70 
ITU International Telecommunication Union. 107

KNN K-Nearest Neighbours. 41

LAOMP Look Ahead Orthogonal Matching Pursuit. 91

LARS Least Angle Regression. 70

LASSO Least Absolute Shrinkage and Selection Operator. 70

LDA Linear Discriminant Analysis. 122

LFR Low Frequency Region. 108

LMS Least Mean Square. 103

LP linear program. 12

LPEC Linear Program with Equilibrium Constraints. 10

LR Logistic Regression. 41,

LSVM Lagrangian Support Vector Machine. 43

MAX FS Maximum Feasible Subsystem Problem. ii

MILP Mixed Integer Linear Program. 8

MIN ULR Minimum Unsatisfied Linear Relation problem. 7

MIN-VOL NMF minimum-volume Nonnegative Matrix Factorization. 126

ML Machine Learning. 1

MMP Multipath Matching Pursuit. 77 
MP Matching Pursuit. 70

MU Multiplicative Update. 124

NB Naive Bayes. 41

NCI National Cancer Institute. 39

NNMF Nonnegative Matrix Factorization. [ii

OMP Orthogonal Matching Pursuit. 24

ORL Olivetti Research Laboratory. 140

PCA Principal Component Analysis. 122

PESQ Perceptual Evaluation of Speech Quality. 107

PFGM Projected Fast Gradient Method. 127

PFP Polytope Faces Pursuit algorithms. 75

PID Pima Indians Diabetes. 51

PRD Percentage Root Mean Square Difference. 91

PSNR Peak Signal to Noise Ratio. 81

PVA Personal Voice Assistants. 102

RIP Discrete Cosine Transform. 64

RLS Recursive Least Mean Square. 103

RNM Random Normalized Matrices. 90 
ROC Receiver Operating Characteristics. 50

ROMP Regularized Orthogonal Matching Pursuit. 77

RSE Relative Squared Error. 107

RSPA Robust Successive Projection Algorithm. 126

RTR Randomized Thermal Relaxation. 22

SCA Sparse Component Analysis. 83

SGP Stochastic Gradient Pursuit. 69

SL0 Smoothed L0. 71

SLSMP Sequential Least Squares Matching Pursuit. 69

SNPA Successive Nonnegative Projection Algorithm. 126

SP Subspace Pursuit. 77

SPA Successive Projection Algorithm. 125

SpAdOMP Sparse Adaptive Orthogonal Matching Pursuit. 69

SpaRSA Sparse Reconstruction by Separable Approximation. 69

StOMP Stage-wise Orthogonal Matching Pursuit. 77

SVD Singular Value Decomposition. 122

SVM Support Vector Machines. 41

TP True Positive. 50 
TSMP Tree Search Matching Pursuit. 69

VQ Vector Quantization. 122

WLDECG wavelet-based, and low-delay ECG. 87 


\section{Nomenclature}

$\delta \quad$ Error tolerance

$<., .>$ Inner product

$\delta_{S} \quad$ Isometric constant

$(.)^{T} \quad$ Matrix transpose operation

$\Phi \quad$ Measurement matrix

$\mu \quad$ Mutual coherence

$(.) \dagger \quad$ Pseudo-inverse

$\Re \quad$ Reconstruction algorithm

$\epsilon \quad$ Small positive constant (Exception: In Eqn. 6.19$), \epsilon$ stands for a sequence converging to zero)

$\Psi \quad$ Sparsifying basis

a Original sparse input

b Right hand side of constraints

$D \quad$ Divergence 
e Non-negative elastic variable

f Input signal (Time domain)

$\hat{\mathbf{f}} \quad$ Recovered Signal (Time domain)

$F_{\sigma}($.$) Smooth function$

H Non-negative coefficient matrix

I Identity matrix

$K \quad$ Length of candidate list

$m \quad$ Number of measurements

$N \quad$ Length of the input signal

$n \quad$ Length of the segment

$P \quad$ Represents matrix rank in NNMF (number of columns of the basis matrix $\mathbf{W}$ )

$r \quad$ Residual vector

$S \quad$ Estimated sparsity based on heuristics

$S_{e} \quad$ Average sparsity error

support Support set

$T \quad$ sparsity of the original signal

V Non-negative matrix

W Non-negative basis matrix (Exception: In Section 6.2, W represents weights in IRWLS) 
winner Index of the best aligned column selected by a sparse recovery algorithm

x Boldfaced lower-case letters denote vectors

X Boldfaced upper-case letters denote matrices

y Measurement vector

Z Objective function 


\section{Chapter 1}

\section{Thesis in a Nutshell}

\subsection{Overview of Contributions}

We live in a world where digital data plays a significant role in shaping our every day lives. These massive volumes of data seem to overwhelm systems in several fields such as healthcare, military, space, and social media [1 1 [ $]$. This data explosion has led to a growing interest in proposing different methods and strategies to address the demands that exist in the digital age such as extracting valuable insights from databases and dimensionality reduction/compression techniques to manage data transmission and data storage.

There is a growing interest in using Machine Learning (ML) algorithms to extract valuable insights from databases in today's world of big data. One central model in ML is classification since it is an important tool for intelligent decision making. Classification is a data analysis technique that is used to find patterns to categorize the data into classes.

In data compression/dimensionality reduction, instead of processing an $n$-long time series which is actually a long vector of discrete time data, it is desired to work 
with a shorter $m$-long vector which contains the same information when $m<<n$. Speaking in signal processing terms, high dimensional $n$-sample signals actually have far fewer than $n$ degrees of freedom; in other words, they are compressible. These high-dimensional data can be well described by considering only $S$ samples, where $S<<n$, that preserve the structure of the signal. Similarly, a high-dimensional data matrix $V_{m \times n}$ can be projected into low dimensional subspaces, $W_{m \times P}$ and $H_{P \times n}$ where $P<<\min (m, n)$ and $(m \times P)+(P \times n)<(m \times n)$ in such a way that certain characteristics of the dataset $\mathbf{V}$ are conserved. Compressing a vector or matrix of data are the two most common cases in data compression [6 10].

Many of the ML/data compression algorithms that are popular today have been around for a long time. For instance, optimization techniques like linear programming date back to the 1940s [12]. However, the modern age of big data has motivated research to find improved solutions in many industries. Maximum Feasible Subsystem (MAX FS) algorithms can help here. They have been shown to have a superior performance in numerous applications such as classification and estimation of sparse solutions for underdetermined linear systems [11,12]. Despite their potentials, recent surveys in data compression and dimensionality reduction, classification, and sparse recovery make no mention of MAX FS approaches. This may be because MAX FS algorithms can be slow. Thus, a main motivation of this thesis is to increase the speed of MAX FS algorithms so that they can be more widely used.

In this work, different strategies are introduced to improve the processing time of the existing MAX FS-based methods while improving/preserving the solution quality. The new strategies developed in the thesis are used to develop more specialized MAX FS algorithms for two important applications:

- binary classification, 
- sparse recovery in compressive sensing (applied to speech and ECG signal recovery),

The MAX FS algorithm concepts are also extended to provide improved solutions for the column subset selection problem in Nonnegative Matrix Factorization (NNMF).

The thesis contributions are:

1. Faster MAX FS algorithms for dense constraint matrices with negligible loss of solution quality.

1.1 Application in binary classification. Faster binary classification algorithm, which provides better results for recall-oriented machine learning tasks such as disease diagnosis compared to widely used classification methods.

1.2 Application in sparse recovery. Faster extensions of the state-ofthe-art MAX FS method for sparse recovery, which require less processing time while maintaining the sparse solution quality.

2. MAX FS algorithms and pipelines for CS sparse recovery to reconstruct more highly compressed signals with higher quality.

2.1 Application in recovery of compressed ECG signals. High quality recovery of highly compressed ECG signals compared to the stateof-the-art CS sparse recovery algorithms.

2.2 Application in recovery of compressed speech signals. High quality recovery of highly compressed speech signals compared to the stateof-the-art CS sparse recovery algorithms.

3. Novel MAX FS-based NNMF algorithms. Three novel MAX FS-based algorithms for NNMF that do not require initialization and that lead to: (1) 
better compression at higher quality, (2) more robustness to outliers, (3) better solution quality for real-world column subset selection problems compared to state-of-the-art.

\subsection{Thesis Organization}

The thesis is organized as follows:

Chapter 2: The Maximum Feasible Subset Problem and MAX FS applications are summarised in Chapter 2, This chapter also covers the existing methods to address the MAX FS problem.

Chapter 3: This chapter identifies gaps in the state-of-the-art MAX FS methods, compressive sensing sparse recovery algorithms, and Non-negative matrix factorization algorithms for dimensionality reduction. Moreover, it details objectives of this work.

Chapter 4: Chapter 4 details two new extensions to Chinneck's original MAX FS solution algorithms for use with dense constraint matrices. Various combinations of the extensions with Method B are evaluated for finding sparse solutions to underdetermined systems of linear equations. The results of the extended methods are compared with that of original Method B in terms of speed.

Chapter 5: This chapter focuses on solving binary classification problems. It provides a summary of the existing binary classification algorithms and details a newly developed MAX FS binary classification algorithm. The performance of the new algorithm is compared with the state-of-the-art MAX FS and non MAX FS methods. 
Chapter 6: This chapter provides an introduction to CS and the existing CS sparse recovery algorithms. A faster version of the state-of-the-art MAX FS method is developed by using the extensions introduced in Chapter 4. Then, the application of the new and existing MAX FS algorithms in CS sparse recovery is investigated in two categories: (i) recovery of compressively sensed ECG signals and (ii) recovery of compressively sensed speech signals. For each category, a new pipeline is developed as an example to indicate how the proposed method can be applied for recovery of compressively sensed signals. In each set of experiments, the performances of the MAX FS algorithms are compared with those of well-known CS sparse recovery methods.

Chapter 7: This chapter focuses on developing and investigating new MAX FS algorithms for solving the NNMF column subset selection problem. It summarizes the widely used methods for solving this problem and details three newly developed MAX FS-based algorithms. The performances of the MAX FS-based algorithms are evaluated in three different sets of experiments.

Chapter 8: The thesis is concluded in Chapter 8 along with suggestions for future work.

\subsection{Publications}

\section{- Refereed journal publications}

* F. F. Firouzeh, J. W. Chinneck, S. Rajan, "Maximum Feasible Subsystem Algorithms for Recovery of Compressively Sensed Speech," IEEE Access., vol.8, no. 1, pp. 82539-82550, 2020.

\section{In Review:}


* F. F. Firouzeh, S. Rajan, J. W. Chinneck, "Fast Maximum Feasible Subsystem Algorithm for Recovery of Compressively Sensed ECG," undergoing first major revision after initial submission to IEEE Transactions on Instrumentation and Measurement.

* F. F. Firouzeh, J. W. Chinneck, S. Rajan, "Faster Maximum Feasible Subsystem Solutions for Dense Constraint Matrices," undergoing major revision after initial submission to Computers and Operations Research Journal.

\section{- Refereed conferences publications}

* F. F. Firouzeh, J. W. Chinneck, S. Rajan, "A Faster MAXimum Feasible Subsystem Algorithm for Binary Classification," Accepted in 2021 IEEE International Symposium on Medical Measurements and Applications (MeMeA).

* F. F. Firouzeh, S. Rajan, J. W. Chinneck, "Recovery of Noisy Compressively Sensed Speech via Regularized Maximum Feasible Subsystem Algorithm," 2021 IEEE International Instrumentation and Measurements Technology Conference (I2MTC).

* F. F. Firouzeh, S. Rajan, J. W. Chinneck, "MAXimum Feasible Subsystem Recovery of Compressed ECG Signals," 2020 IEEE International Symposium on Medical Measurements and Applications (MeMeA), Bari, Italy, June 1-3, pp. 1-5, 2020.

* F. F. Firouzeh, M. Abdelazez, S. Salsabili, S. Rajan, "Improved Recovery of Compressive Sensed Speech," 2020 IEEE International Instrumentation and Measurements Technology Conference (I2MTC), Dubrovnik, Croatia, May 25-28, pp. 1-5, 2020. 


\section{Chapter 2}

\section{The Maximum Feasible Subsystem Problem (MAX FS)}

Finding the maximum cardinality feasible subsystem of an infeasible set of linear constraints is known as the Maximum Feasible Subsystem problem (MAX FS) [13]. Based on [14], MAX FS is the same as the Minimum Unsatisfied Linear Relation problem (MIN ULR). MIN ULR finds the minimum number of constraints to remove such that the remaining constraints constitute a feasible solution for the original infeasible system.

MAX FS is known as an NP-hard problem [15 17]. Finding a maximum feasible subsystem plays a significant role in a wide variety of applications in fields including machine learning [18], misclassification minimization [19], training of neural networks [14], telecommunication [20], and computational biology [21]. Therefore, there is a high demand imposed upon heuristic algorithms to obtain high quality solutions in an acceptable amount of time.

In contrast to the problems with a set of nonlinear constraints, problems with a linear set of constraints are more tractable. Consequently, there are some good 
heuristics that provide high quality MAX FS results when all of the constraints in the infeasible set are linear. In the next section, an overview of the existing MAX FS heuristics that can be applied for the mentioned applications is presented.

\subsection{Existing MAX FS Methods for Sets of Linear Constraints}

In 2019, Chinneck 22 provided a survey on the existing algorithms for the MAX FS problem. In this section, a brief summary of the different categories of the existing MAX FS methods, which are shown in Fig. 2.2 is provided. Note that MAX FS and MIN ULR are the same problem in this work and the terms will be used interchangeably.

\subsubsection{Provably Optimal Formulations}

MAX FS can be formulated for exact solution. Despite this fact, MAX FS is known to be NP-hard; hence the exact solution is only applicable for relatively small instances. Therefore, exact formulations are not practical for a wide variety of problems since they require exponential time to run.

An exact solution can be obtained via Mixed Integer Linear Program (MILP) which is formulated as follows:

$$
\begin{gathered}
\min Z=\sum_{i} y_{i} \text { s.t. } \\
\boldsymbol{a}_{i} \mathbf{x} \leq b_{i}+M y_{i} \quad \text { for all constraints } i \text { of type } \leq \\
\boldsymbol{a}_{i} \mathbf{x} \geq b_{i}-M y_{i} \quad \text { for all constraints } i \text { of type } \geq \\
\boldsymbol{a}_{i} \mathbf{x}=b_{i}+M y_{i}^{\prime}-M y_{i}^{\prime \prime} \text { for all constraints } i \text { of type }=
\end{gathered}
$$




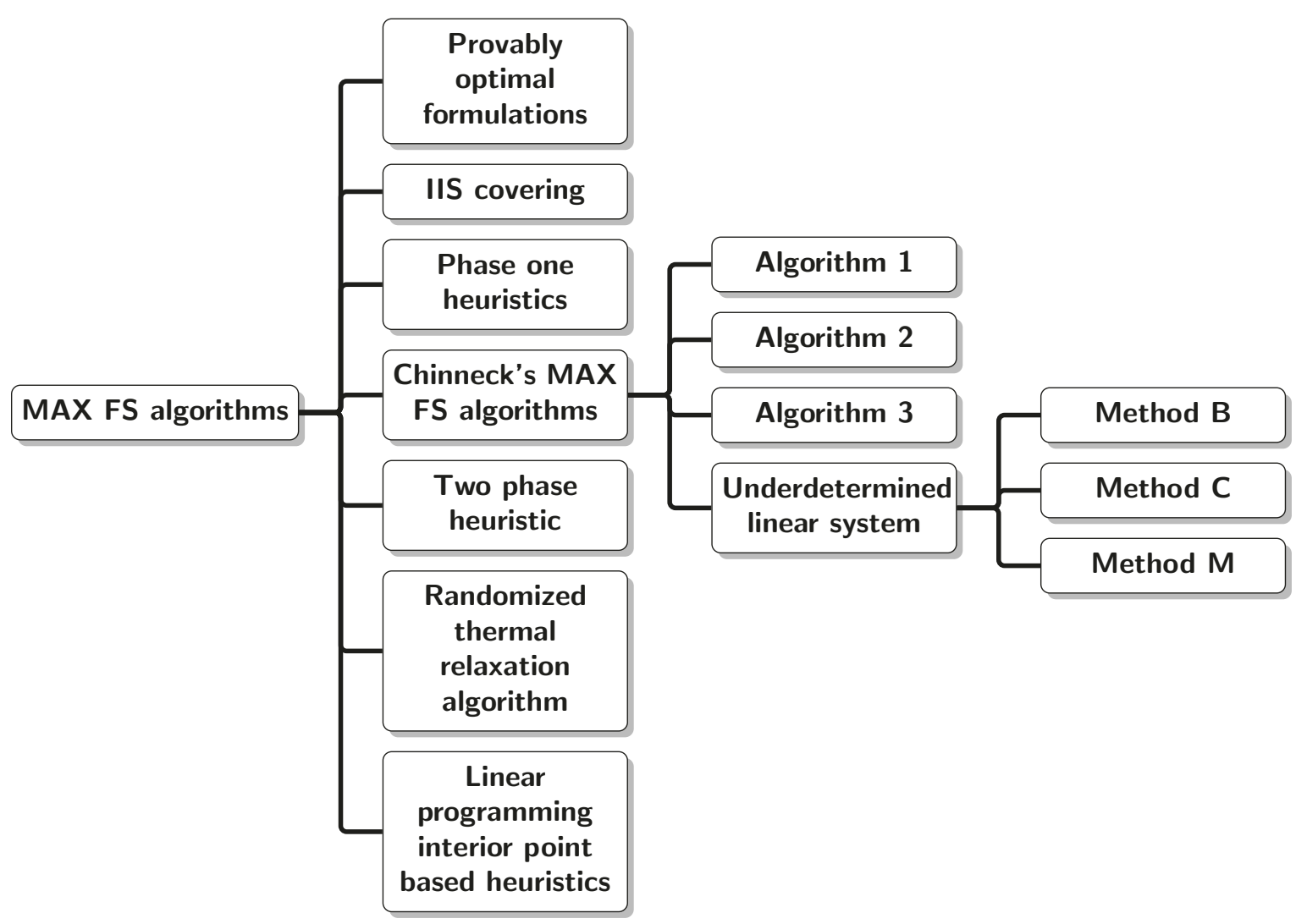

Figure 2.1: Different categories of the existing MAX FS methods.

where the $y, y^{\prime}$, and $y^{\prime \prime}$ are binary variables, and $M$ is the usual "big- $M$ " large positive value. Also, $\mathbf{x}$ and $\mathbf{A}$ are an unknown column vector and the $m \times n$ constraint matrix, respectively. Let $\boldsymbol{a}_{i}$ represent the $i^{\text {th }}$ row of A. As shown above, all variable bounds are included in the set of row constraints. This formulation has so many deficiencies: (1) it has too many row constraints (i.e. $m$ original constraints, one constraint for each singly-bounded variable, and two for each doubly-bounded variable), (2) it has too many variables (i.e. $n$ original variables, one binary variable for each inequality constraint and single-bounded variable, and two binary variable for each equality constraint and double-bounded variable), and (3) the large value of $M$ leads to numerical instability, although a small value of $M$ leads to incorrect solutions.

In 2003, Amaldi 23] proposed an exact formulation for the MAX FS problem as a 
Linear Program with Equilibrium Constraints (LPEC). This formulation is provided in Eqn. (2.2).

$$
\max \sum_{i=1}^{m} y_{i} \quad \text { s.t. } \quad y_{i} \boldsymbol{a}_{i} \mathbf{x}\{\leq, \geq,=\} \quad y_{i} b_{i}, \quad y_{i} \in\{0,1\}
$$

where $\boldsymbol{a}_{i}$ is the $i^{t h}$ row of the matrix A. The objective function will drive any binary variables, $y_{i}$, to one if the equation is included in the feasible subset. The aforementioned formulation constitutes a nonlinear global optimization problem. This problem can be tackled via global solvers, or by standard NLP local solvers, hence only approximate solutions can be obtained.

Different variations of the LPEC formulation have been proposed such as 24 27. The best of the LPEC variations is MISMIN algorithm [27]. However, Chinneck 11] experimentally showed that the MISMIN is less accurate than the MAX FS solution heuristics that will be discussed in Section 2.1.4. Therefore, the exact formulations, MILP and LPEC, are theoretically able to find exact solutions, but nonetheless, they are too slow and unreliable in practice.

\subsubsection{Irreducible Infeasible Sets (IIS) Covering}

In [28], a method is proposed for enumerating Irreducible Infeasible Sets (IIS) in an infeasible system of linear inequalities. An IIS is a subset or set of constraints which is infeasible; however, it is feasible if any one member is removed. In the IIS Covering method, a standard set-covering problem is solved to find the minimum IIS cover when all of the IISs are known. The set-covering problem, which is solved by binary integer programming, is the process of finding the smallest set of elements such that each subset contains at least one of the chosen elements. In the MAX FS problem, the elements and the sets are the constraints and the IISs in the model, respectively. 
Some challenges are associated with this method: (1) the number of IISs can be exponential in the size of the model as discussed in [29], (2) the set-covering problem is a difficult integer programming problem, and (3) the method can be applied only to inequalities. Therefore, this method is impractical.

Researchers tried to address the mentioned problems in different works. For instance, Parker and Ryan [30] proposed a method for solving the problem of generating IISs. Moreover, Pfetsch [31, 32 increased the speed of the set cover solutions by applying various heuristic cuts. Also, Pfetsch described a heuristic for generating new IISs and that was the best method available at the time.

\subsubsection{Phase one Heuristics}

The Phase one procedure searches for an initial feasible basis in the simplex-based linear programming algorithms. The feasibility status of the model is unknown beforehand. Therefore, a relaxed or elasticized version of the model is used to obtain a feasible solution for the elasticized model, even if there is no feasible solution for the original model. To construct the elasticized model, non-negative elastic variables, $e_{i}$, are added to each constraint to allow them to be violated. For instance, all $=$ and $\geq$ constraints are elasticized in the standard linear programming Phase one procedure [33]. In the elastic version, all elasticized constraints are allowed to be violated to find the "feasible" solution for the original model. Therefore, each nonzero elastic variable indicates a violated constraint in the original problem. Moreover, two versions of elastic programs are: (1) Standard elastic program and (2) Full elastic program. In a standard elastic program, only row constraints are elasticized. In a full elastic program, all constraints including rows and column bounds are elasticized. As indicated in Table 2.1, every inequality constraint is elasticized by adding a nonnegative elastic variable, and every equality constraint is elasticized by adding two 
Table 2.1: Constraint elasticizing by using non-negative elastic variables.

\begin{tabular}{||cc||}
\hline Nonelastic Constraint & Elastic Version \\
\hline \hline$a_{i} \mathbf{x} \geq b_{i}$ & $a_{i} \mathbf{x}+e_{i} \geq b_{i}$ \\
\hline$a_{i} \mathbf{x} \leq b_{i}$ & $a_{i} \mathbf{x}-e_{i} \leq b_{i}$ \\
\hline$a_{i} \mathbf{x}=b_{i}$ & $a_{i} \mathbf{x}+e_{i}^{+}-e_{i}^{-}=b_{i}$ \\
\hline
\end{tabular}

elastic variables, $e_{i}^{+}$and $e_{i}^{-}$. Therefore, each constraint can stretch in either direction as much as required.

The measure of improvement is the decrease in the total infeasibility, i.e. the sum of the constraint violations, $Z=\min \sum_{i} e_{i}$. So, the objective function, $Z$, minimizes the total infeasibility. If the model is infeasible, one or more elastic variables are greater than zero, which means the minimum value of $Z$ is greater than zero, and hence the corresponding constraint is not satisfied at the solution. Then, those violated constraints with nonzero elastic variables constitute the heuristic estimate of the MIN ULR set.

Linear Programming ( $\mathrm{LP}$ solvers are utilized to obtain the model solution. As different LP solvers consider different crash start heuristics, different bounds adjustments, etc., then different lists of nonzero elastic variables are estimated. Therefore, the solution is not unique; however, any obtained list is a valid heuristic solution of the MIN ULR problem.

\subsubsection{Chinneck's MAX FS Algorithms}

In this section, different versions of Chinneck's MAX FS algorithms are illustrated. In general, at each iteration of the heuristics, there is a list of constraints that are candidates for removal. Each candidate is temporarily removed from the model, a measure of improvement is calculated, and the candidate is returned to the model. After all of the candidates in the list have been tested, the constraint that provided 
the best measure of improvement is permanently removed. The process stops when the remaining constraints admit a feasible solution. More details of each of Chinneck's methods are presented below.

\subsubsection{Algorithm 1}

In 1996, an effective polynomial time heuristic was proposed by Chinneck [34] to solve the MAX FS problem. The algorithm uses an elastic setup and it works by removing constraints one at a time to solve an LP, $\mathbf{A}_{m \times n} \mathbf{x}_{n \times 1}\{\geq,=, \leq\} \mathbf{b}_{m \times 1}$. The removed constraints are the Cover Set, which is the estimated MIN ULR solution.

A simplified version of Algorithm 1 is shown in Fig. 2.2. To start, CoverSet is empty. After elasticizing and solving the first elasticized LP, the first candidate list is obtained. Each candidate in the CandidateSet is tested and its corresponding $Z$ is obtained. The constraint whose removal provides the smallest $Z$ is removed and added to the Cover Set. The process continues until $Z$ reaches zero or a stated tolerance.

Algorithm 1 can be slow due to the necessity of solving an LP for each candidate. Chinneck 11] proposed a way of reducing the length of the candidate list: it is sorted in descending order of constraint absolute sensitivity, and then the list is truncated after the first $k$ candidates. This is denoted as Algorithm 1(k).

Chinneck [11] also proposed different ways of creating the list of candidates, deemed "Algorithm 2" and "Algorithm 3". These are explained in the following sections. The idea of the truncated list can also be applied to these methods.

\subsubsection{Algorithm 2}

Chinneck's Algorithm $2[11]$ creates the candidate list in a different way. Instead of considering the constraints to which the elastic objective function is sensitive, 


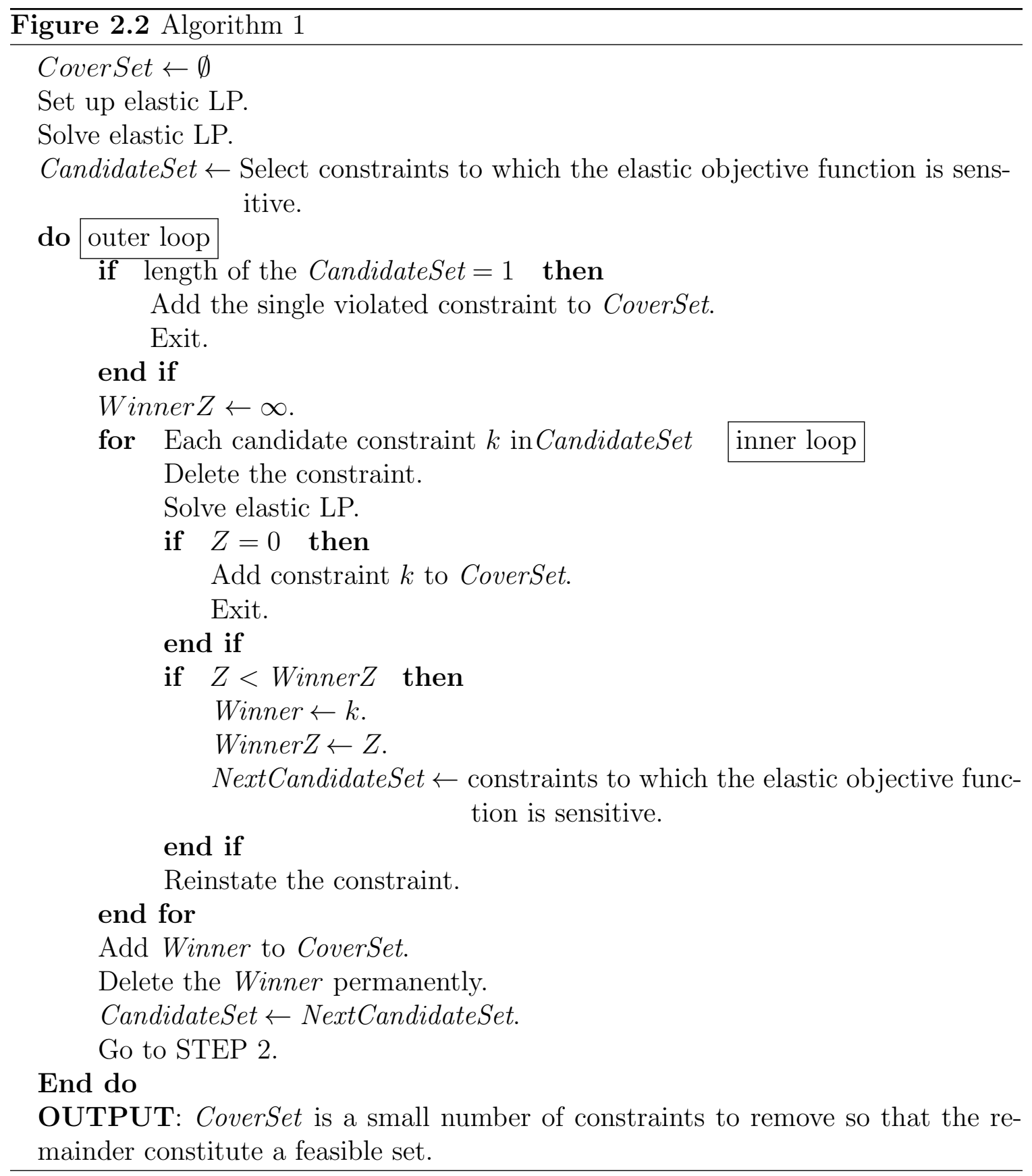


the CandidateSet consists only of the violated constraints, with their figure of merit calculated as the product:

$$
\text { Product : } \mid \text { constraint violation }|\times| \text { constraint sensitivity } \mid
$$

Algorithm 2 requires the full elasticization of the original model. In fact, all the constraints and variable bounds are elasticized in this case. The value of the elastic variable and the reduced cost of the variable associated with a violated constraint provide the "constraint violation" and "constraint sensitivity", respectively. For equality and range constraints, for which there are two elastic variables, the maximum value of the two elastic variables gives the constraint violation. As all of the violations are relaxations of the constraint, the absolute value of the constraint sensitivity is considered regardless of the constraint sense $(\geq,=, \leq)$. The CandidateSet in Algorithm 2 is obtained as follows:

- Find the product based on Eqn. (2.3) and sort them in order from the largest to the smallest value.

- Select the top $k$ components of the ordered list to create the CandidateSet.

Algorithm 2 also called "Algorithm 2(k)" since a candidate list of length $k$ is considered in this method. Algorithm 2( $\infty)$ refers Algorithm 2 with an unlimited list length. When $k=1$ only a single candidate is taken from the list; this is referred to as the max product version of Algorithm 2(1).

\subsubsection{Algorithm 3}

By not considering constraints that are tight but not violated, Algorithm 2 sometimes misses some useful candidates. Algorithm 3 corrects this shortcoming by considering 
both the violated constraints (using the product as in Algorithm 2) and the tight but satisfied constraints (as in Algorithm 1). The CandidateSet in Algorithm 3 is constructed as follows:

- Find the product for each violated constraint based on Eqn. (2.3) and sort them in order from the largest to the smallest value.

- Select the top $k$ components of the ordered list to generate the CandidateSet.

- Find |constraint sensitivity| for the satisfied constraints and arrange them in order from largest to smallest.

- Select the first $k$ elements of the ordered list, then add them to the bottom of the CandidateSet.

Algorithm 3 also called "Algorithm 3(k)". In Algorithm 3(k), $k$ refers to the length of each of the two mentioned lists. The list has a total length of $2 k$.

\subsubsection{Underdetermined Linear Systems}

As mentioned earlier, finding a sparse solution (i.e. sparse solution is a solution with small number of nonzeros) for an underdetermined system can be considered as MAX FS problem: find a MAX FS solution for the system $\mathbf{A x}=\mathbf{b}, \mathbf{x}=\mathbf{0}$ where only constraints in the set $\mathbf{x}=\mathbf{0}$ can be removed in order to achieve feasibility. The following three variations of Chinneck's method, namely Method B, Method C, and Method M have been developed recently [35] specifically for finding sparse solutions to underdetermined linear systems.

Two different methods are used to force variables to zero. The first uses the fact that LP solvers normally assign lower and upper bounds to all variables: $l_{j} \leq x_{j} \leq u_{j}$. Unrestricted variables have $-\infty \leq x_{j} \leq+\infty$. Variables can be forced to zero by 
setting $l_{j}^{l}=u_{j}=0$. A second way is by including an explicit elastic variable zeroing constraint $x_{j}+e_{j}^{+}-e_{j}^{-}=0$ where $e_{j}^{+} \geq, e_{j}^{-} \geq 0$; the elastic objective function then encourages $x_{j}$ towards zero.

LP solvers also normally have upper and lower bounds on the constraint function values: $b_{i}^{l} \leq g_{i}(x) \leq b_{i}^{u}$ where $g_{i}(x)$ is the $i^{\text {th }}$ constraint. This makes it straightforward to formulate a system for finding approximate solutions to the equations, which helps to control the error in the sets of the linear constraints. By defining an acceptable positive equation error tolerance $\delta$, each linear equation as follows: $b_{i}^{l}-\delta \leq g_{i}(x) \leq$ $b_{i}^{u}+\delta$.

The algorithms may return supports with superfluous members. Some of these can be removed by a postprocessing step [12]. First, force all non-support variables to zero (or remove from the model). Next, run through the list of support members, temporarily forcing each to zero in turn; if there is a feasible solution, then that support member can be removed from the support set.

Finally, while the algorithms return the support, they do not provide their values. These are found in a final LP solution. The system $\mathbf{A}^{\prime}$ is constructed, which contains only the columns of $\mathbf{A}$ corresponding to the support. Also, each $\mathbf{x}_{j}$ is substituted by the difference of two nonnegative variables: $u_{j}-v_{j}$. Then, the support is obtained by solving the LP:

$$
\min Z=\sum_{j}\left(\mathbf{u}_{\mathbf{j}}+\mathbf{v}_{\mathbf{j}}\right) \quad \text { s.t. } \quad \mathbf{A}^{\prime}(\mathbf{u}-\mathbf{v})=\mathbf{b}, \quad \mathbf{u} \geq \mathbf{0}, \mathbf{v} \geq \mathbf{0}
$$

The support values are recovered by reversing the change of variables: $x_{j}=u_{j}-v_{j}$. The three MAX FS algorithms for underdetermined systems are illustrated in the following sections.

Method B: The Method B variant is summarized in Fig. 2.3. It uses the change 
of variables LP formulation:

$$
\min Z=\sum_{j}\left(\mathbf{u}_{\mathbf{j}}+\mathbf{v}_{\mathbf{j}}\right) \quad \text { s.t. } \quad \mathbf{A}(\mathbf{u}-\mathbf{v})=\mathbf{b}, \quad \mathbf{u} \geq \mathbf{0}, \mathbf{v} \geq \mathbf{0}
$$

Here $\mathbf{A}$ is of size $m \times 2 n$, and this LP has $m$ constraints in $2 n$ variables. The algorithm follows the general MAX FS algorithm logic with these features:

- The initial objective function coefficients for all $u_{j}$ and $v_{j}$ are 1.0.

- Candidate variables are those having an objective function coefficient of 1.0 and a magnitude greater than a stated tolerance. Candidates are sorted in decreasing order by the magnitude of $|\mathbf{u}-\mathbf{v}|$. The length of the list of candidates is controlled by a parameter namely ListLength, typically set to 1,7 , or unlimited.

- Candidates are tested by reducing the objective function coefficients of the $u_{j}$, $v_{j}$ pair to zero.

- The objective function coefficients of the winning $u_{j}, v_{j}$ pair are reset to 0.1 . This encourages support members to take on zero values if they are able, hence reducing the need for postprocessing.

\section{Method C:}

Method C uses explicit elastic variable zeroing constraints $x_{j}+e_{j}^{+}-e_{j}^{-}=0$, where $e_{j}^{+}$and $e_{j}^{-}$are nonnegative, resulting in the following LP:

$$
\begin{gathered}
\min Z=\sum_{j}\left(e_{j}^{+}+e_{j}^{-}\right) \text {s.t. } \\
{\left[\begin{array}{ccc}
\mathbf{A} & \mathbf{0}_{m \times n} & \mathbf{0}_{m \times n} \\
\mathbf{I} & \mathbf{I} & -\mathbf{I}
\end{array}\right]\left[\begin{array}{c}
\mathbf{x} \\
\mathbf{e}_{\mathbf{j}}^{+} \\
\mathbf{e}_{\mathbf{j}}^{-}
\end{array}\right]=\left[\begin{array}{c}
\mathbf{b}_{m \times 1} \\
\mathbf{0}_{n \times 1}
\end{array}\right], \quad \mathbf{e}_{j}^{+} \geq 0, \quad \mathbf{e}_{j}^{-} \geq 0,}
\end{gathered}
$$




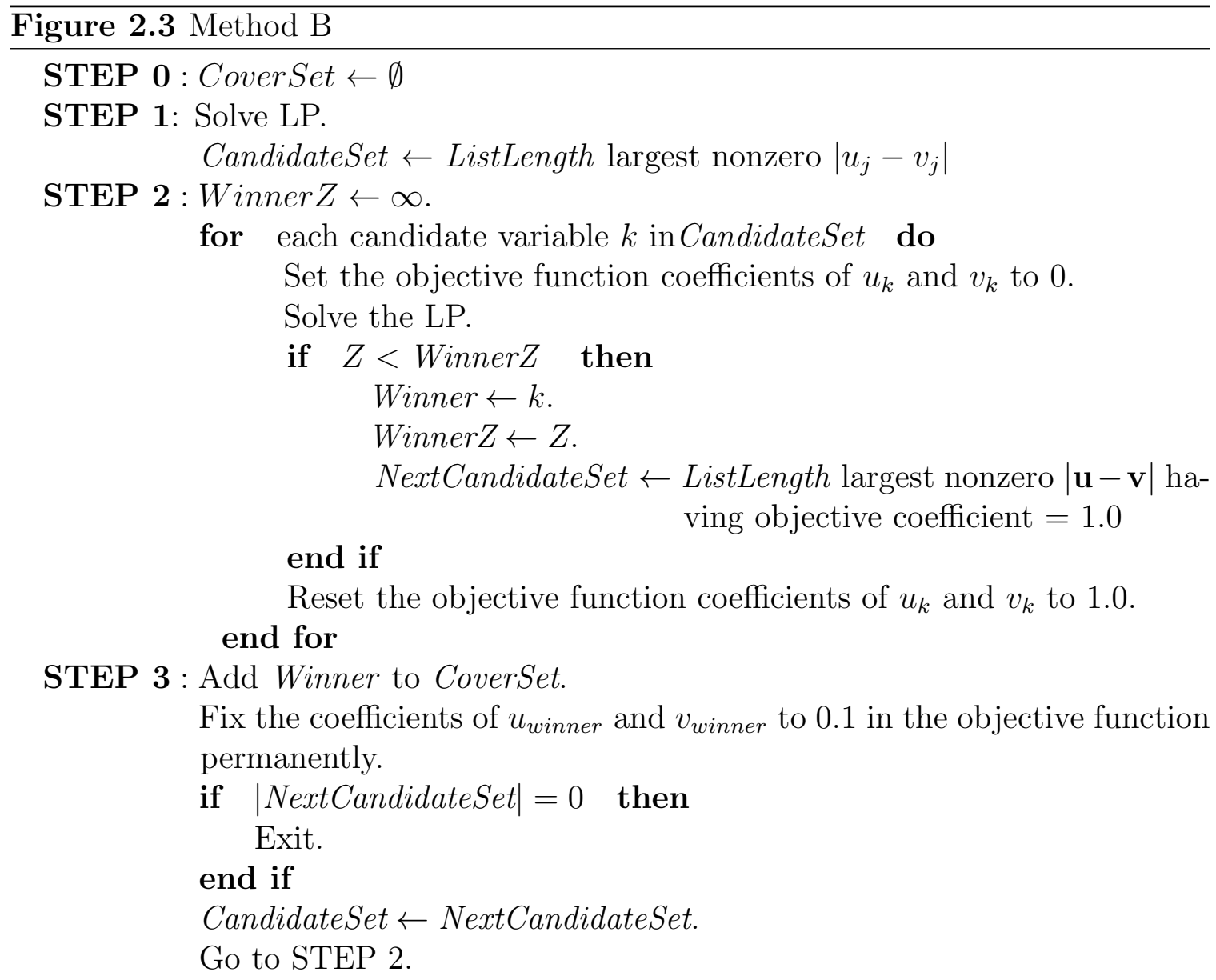

OUTPUT: CoverSet is small number of nonzeros forming a support for the system of equations. 
where $\mathbf{A}$ and $\mathbf{I}$ are of size $m \times n$ and $n \times n$, respectively. The LP has $m+n$ constraints in $3 \times n$ variables. The main features of Method $\mathrm{C}$ are:

- There are two lists of candidates, one based on the magnitude of the nonzeros, CandidatesNZ, and the other based on the sensitivity of the elastic objective function to the variable zeroing constraint, CandidatesSens. Both lists are sorted in decreasing order of magnitude and the top ListLength candidates from each list are taken.

- A variable $k$ is added to the support set by removing the elastic variables in its zeroing constraint from the objective function.

Method $\mathrm{C}$ is summarized in Fig. 2.4.

Method M: Method M combines Method B with Basis Pursuit (BP) (refer to Section 6.2 to find out details about BP). BP is very efficient if there is a very sparse solution then solving Eq. (2.5) once (which is BP) is likely to get the right solution. BP typically returns either a sparse solution correctly, or it returns $\mathbf{x}$ with a larger sparsity (i.e. Sparsity refers to number of nonzero elements) equal to or close to $m$. It is thus easy to recognize when BP has succeeded. M applies the more time-consuming Method B only if BP fails. M assumes BP failure if sparsity of the BP solution is greater than a defined threshold such as $m-3$, in which case it runs Method B.

\subsubsection{Two Phase Heuristics}

Amaldi et al. [36] developed a two-phase method based on the assumption that the solution of the exact big-M Mixed-Integer Linear Programming is effective for a not too large problem. In phase one of this method, the goal is to find a consistent set that cannot be violated during phase two. In phase two, the goal is to use an exact 


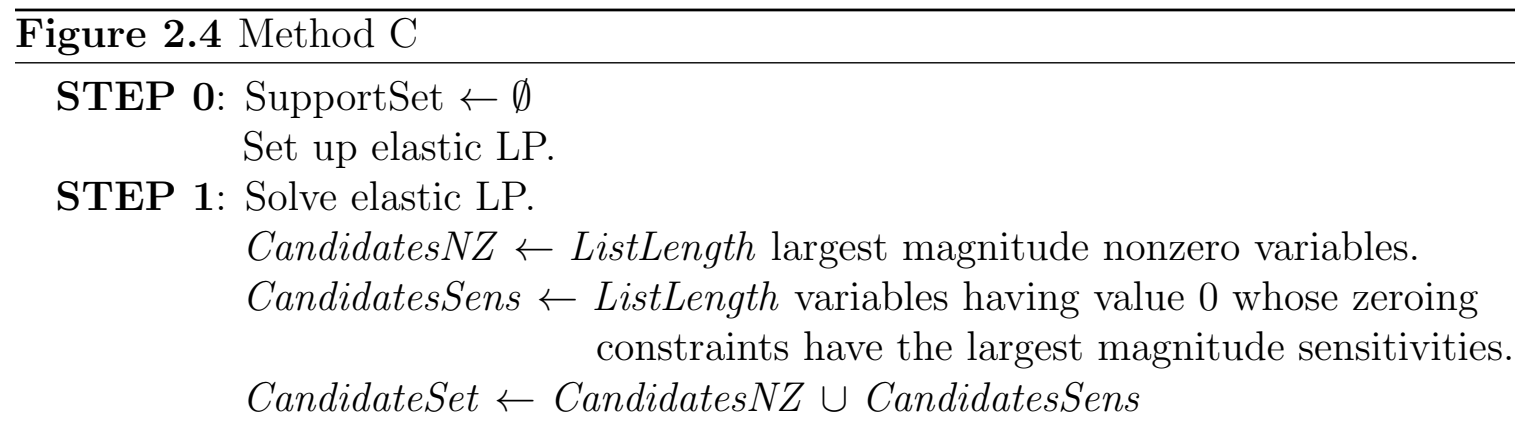

STEP 2: Winner $Z \leftarrow \infty$.

for each candidate $k$ in CandidateSet do

Set the objective function coefficients of $e_{k}^{+}$and $e_{k}^{-}$to 0 .

Solve elastic LP.

if $Z=0$ then

Add variable $k$ to SupportSet. Exit.

end if

if $\quad Z<$ Winner $Z$ then

Winner $\leftarrow k$.

Winner $Z \leftarrow Z$.

NextCandidates $N Z \leftarrow$ ListLength largest magnitude nonzero variables, excluding support variables and $k$.

NextCandidatesSens $\leftarrow$ ListLength non-support variables having value 0 whose zeroing constraints have the largest magnitude sensitivities.

NextCandidateSet $\leftarrow$ NextCandidates $N Z \cup$ NextCandidatedatesSens

\section{end if}

\section{end for}

Set the objective function coefficients of $e_{k}^{+}$and $e_{k}^{-}$to 1 .

STEP 3: Add Winner to SupportSet.

Set the objective function coefficients of $e_{\text {winner }}^{+}$and $e_{\text {winner }}^{-}$to 0 permanently.

CandidateSet $\leftarrow$ NextCandidateSet.

Go to STEP 2.

OUTPUT: SupportSet is a small number of nonzeros forming a support for the system of equations. 
big-M mixed-integer linear programming to enlarge the defined set of constraints in phase one by adding as many constraints as possible without causing infeasibility.

The two-phase algorithm is quite effective in large instances (i.e. up to about 20,000 constraints). Also, as the phase two of this method is much smaller than that of the original problem, big-M mixed-integer linear programming often succeeds in a reasonable amount of time.

\subsubsection{Randomized Thermal relaxation Algorithms}

Amaldi et al. [37] proposed a heuristic method called Randomized Thermal Relaxation (RTR. This method, which is a combination of modified ideas from Tabu search 38 and projection algorithms [39 provides an effective solution for large scale linear models (i.e. consisting up to about 400,000 linear inequalities). In the RTR method, a single constraint is chosen at random. Based on the idea in Tabu search, if the selected constraint is satisfied, then no update takes place, but if it is violated, then the closest point that satisfies the constraint is calculated. The main operation in projection methods is finding the feasibility vector between the current point and the closest point that satisfies the constraint. The size of the update is controlled by the 'temperature' parameter. As the algorithm runs, the temperature parameter decreases and the updates become shorter.

By adjusting the three main elements, different variations of this method can be developed. The key elements are: (1) the rule for selection a constraint for the update, (2) the rule for estimating the update size, and (3) the rule by which the probability of updating a violated constraint is set. 


\subsubsection{Linear Programming Interior Point-based heuristics}

In [40], Meller et al. utilized the properties of interior-point LP solvers to estimate the solution for MAX FS for extensive sets of linear inequalities. In the absence of an objective function, the interior point method obtains a point that is close to the analytic center due to the barrier function that tends to push the solution point away from satisfied constraints and towards the center of a polytope created by a set of linear inequalities. The proposed method by Meller et al. can be summarized in three steps: (1) consider an initial point which satisfies some subset of the linear inequalities, (2) update the point by approximation an interior point solution for the set of satisfied constraints, and (3) iterate the process until no additional constraints are satisfied by the new interior point solution.

\subsection{Discussion and Comparison}

Pfetsch [31,32] compared his algorithms with Chinneck's for the classification problem. In most cases, the comparison showed that both mentioned MAX FS heuristics obtained an identical solution, but Pfetsch's method required more time to find the solution [22]. The high accuracy of Chinneck's algorithm on classification is discussed in [41]. Chinneck's algorithm is tunable; hence, it is capable of finding the separating hyperplanes for different purposes and characteristics by adjusting the strategy of removing candidates at each iteration. For instance, it can find (1) hyperplanes that emphasize the accuracy of one type over the other, or (2) protect minority classes. Moreover, Chinneck's algorithm can select features and the separating hyperplanes simultaneously.

In the application of finding a sparse solution of an underdetermined system, 
Jokar and Pfetsch [12] compared a variation of Chinneck's algorithm, Method B, to a number of others such as Basis Pursuit (BP), Orthogonal Matching Pursuit OMP, Greedy Orthogonal Matching Pursuit (GOMP), a branch-and-cut MILP, and several nonlinear MAX FS methods and concluded that Chinneck's algorithm provides the best results overall. According to [12], the critical sparsity of the existing sparse recovery algorithms is smaller than that of Method B. Consequently, the existing algorithms which are not based on MAX FS are only able to return a sparse solution of an underdetermined systems under certain limited conditions efficiently. Generally, there is a very sparse solution. If the mentioned condition is not met, the number of nonzeros of the recovered solution is much larger than the input number of nonzeros.

According to 22], Chinneck's algorithm can be considered as the most effective method to solve the MAX FS problems. However, there may be opportunities for improving the speed further. This method has been used in a wide variety of applications and it is very effective for practical problems, especially in finding the sparse solution of underdetermined systems [12]. BP Although the existing Chinneck's algorithms are effective, their speed can be further improved since they need to solve one LP to test each candidate in the list. As shown in Fig. 2.2, Chinneck's algorithms remove constraints from the original set one by one until what remains is the heuristic MAX FS solution; the removed constraints constitute the heuristic MIN ULR solution. An inner loop assesses candidate constraints, and an outer loop removes the single constraint chosen by the inner loop. Limiting the length of the candidate list is helpful, but there are still two bottlenecks: evaluating multiple candidates in the inner loop, and removing just a single constraint in the outer loop. This thesis extends Chinneck's algorithms to provide major improvements in solution speed. Chapter 4 explains the details of the extended algorithms and their potential applications. 


\section{Chapter 3}

\section{Problem Statement}

The primary objective of this work is to develop algorithms based on solution methods for the MAX FS problem in order to: (1) increase the speed of the state-of-the-art MAX FS methods while improving/maintaining accuracy, and (2) use these algorithms to improve performance in data compression, classification, and sparse recovery. This thesis focuses on three applications, namely binary classification, sparse recovery in Compressive Sensing (CS) and Non-Negative Matrix Factorization (NNMF) using different synthetic and real-world datasets such as speech, Electrocardiogram (ECG), biological datasets, and images.

Many problems in the areas of signal processing such as speech processing, biomedical engineering and image processing can be converted to instances of the MAX FS problem and hence solved by existing MAX FS algorithms. While effective in solving numerous difficult NP-hard problems, a drawback of the existing MAX FS heuristics is that they can be slow. Therefore, the first problem addressed in this thesis is improving the speed of the existing MAX FS algorithms for certain situations, without reducing the quality of the solutions.

The second major problem is determining how to adapt the MAX FS algorithms to 
provide better results in a variety of sparse recovery and classification tasks. Specific problems include using the improved MAX FS algorithms (i) in the CS recovery phase to recover more highly compressed speech and ECG signals with greater quality, and (ii) in binary classification.

The third major problem is the extension of the main MAX FS idea to nonnegative matrix factorization, where direct application of MAX FS algorithms will not work. This requires the development of novel algorithms that generalize from the core ideas of existing MAX FS heuristics.

\subsection{Deficiencies of the State-of-the-Art}

This thesis investigates the idea that MAX FS-based algorithms can improve some of the deficiencies of the start-of-the-art in several applications. Although algorithms based on solution methods for the MAX FS problem are already known to provide good accuracy for binary classification, they are slow. Therefore, this thesis will focus on improving their speed while maintaining/improving accuracy.

In CS, fewer measurements leads to more compression, and therefore, to transmission and storage of less data. For greater compression in CS, the critical sparsity 1 of a signal recovery method must be higher in order to achieve a stable recovery. However, the critical sparsity of the well-known sparse recovery algorithms is not large enough to allow sufficient signal compression with high quality recovered signal. Existing algorithms can recover the input signal precisely with high probability only when the input signal is very sparse, and it is not compressed much; otherwise, the recovered signal is of low quality. In practical applications, a sparse solution is needed even if these conditions are not met. In practice, the input signal sparsity

\footnotetext{
${ }^{1}$ Critical sparsity is the maximum sparsity at which a sparse recovery algorithm returns sparse solutions reliably (refer to Section 4.2 .2 to find more details).
} 
is not known during the compression and recovery phases; it is either estimated or assumed. The quality of the output signals recovered from highly compressed signals is still an area that needs more improvement. More details about inefficiencies of the existing algorithms are available in Section 6.2

Non-negative matrix factorization algorithms suffer from some deficiencies as explained in Section 7.2. Almost all algorithms require the initialization step, which adds one more step to the NNMF process. Most of the NNMF algorithms assume that rank is specified a priori. Finding the optimal rank in NNMF is still an unsolved problem. Moreover, there is a need for an algorithm which is robust to outliers in column selection problems and provides better solution quality. 


\section{Chapter 4}

\section{New MAX FS Algorithms for Dense Constraint Matrices}

As explained in Chapter 2, Chinneck proposed the state-of-the-art algorithms to solve MAX FS problem. Although Chinneck's methods are effective, they can be slow for large problems. Despite this issue, many applications, such as classification, finding the data depth, radiation treatment planning, protein folding, digital video broadcasting, automated test assembly, customized web page ranking, sparse solution of underdetermined systems, the minimum number of feasible partitions problem, can be cast as MAX FS problem 222. In this chapter, the existing heuristics are extended for the case of dense constraint matrices to greatly increase their speed while preserving or improving solution quality. The following sections detail the extended and new MAX FS algorithms. 


\subsection{Extensions for Dense Constraint Matrices}

One of the contributions of this research is extending Chinneck's algorithms to provide major improvements in solution speed for the case of dense constraint matrices. This improvement is based on the observation that dense constraint matrices provide multiple similar candidates for removal during the MAX FS solution process. The superior performance of the extended algorithms on two applications that have dense constraint matrices, namely binary classification, and sparse recovery in compressive sensing are detailed in Chapter 5 and Chapter 6, respectively. In the classification problem, all attribute values are specified for all data points, and the constraint matrix $\mathbf{D}$ is dense. For several types of compressive sensing (CS) measurement matrices A, such as random matrices of various types, $\mathbf{A}$ is dense, and thus suitable for the extended algorithms introduced in this research. In general, the new extensions can be used for (i) overdetermined systems of linear inequalities in unbounded variables for classification, and (ii) underdetermined systems of linear equations in unbounded variables for CS:

- Overdetermined systems of linear inequalities in unbounded variables for classification: According to Chinneck [11, [14, Parker 28, and Silva 42, MAX FS can be used for the classification problem. Separating data points of two different types in an n-dimensional space is a basic operation in machine learning which can be transformed into the MAX FS problem as follows:

Given: a training set of $I$ data points $(i=1, \ldots, I)$ in $J$ dimensions $(j=$ $1, \ldots, J)$. The class of each point is known (either type zero or type one).

Define: a constraint for each data point of type zero and type one:

- For points of type zero: $\sum_{j} d_{i j} w_{j} \leq w_{0}-\epsilon$ 
- For points of type one: $\sum_{j} d_{i j} w_{j} \geq w_{0}+\epsilon$

where $d_{i j}$ indicates the value of attribute $j$ for point $i$. Also, $d_{i j}$ are known constants, $\epsilon$ is a small positive constant and variables, $w_{j}$, are unrestricted, where $(j=0, \ldots, J)$. For the mentioned classification problem, the MAX FS solution is a separating hyperplane, $\sum_{j} d_{i j} w_{j}=w_{0}$, which correctly classifies the largest number of the given data points.

\section{- Underdetermined systems of linear equations in unbounded variables}

for CS: Finding a sparse solution for an underdetermined feasible system can be cast as an instance of MAX FS, as discussed in [12]. The sparse solution problem is:

Given: a system of linear equations, $\mathbf{A x}=b$.

Find: $\min \|\mathbf{x}\|_{0} \quad$ s.t. $\quad \mathbf{A x}=\mathbf{b}$.

where $\|x\|_{0}$ is the number of nonzeros in $\mathbf{x}$. The number of rows $m$ in $\mathbf{A}$ is smaller than the number of columns $n$, so the system is underdetermined and has multiple solutions. Finding the sparsest solution is the same as searching for the largest cardinality feasible subset of an infeasible linear system. The details of converting this problem into the MAX FS problem are discussed later.

As discussed in Chapter 5, finding a sparse solution of an underdetermined system serves a crucial role in the area of compressive sensing. However, the compressive sensing literature such as 4345$]$ does not mention MAX FS methods. But, Jokar and Pfetsch [12] and Chinneck [22] pointed out the potential of using MAX FS in the sparse recovery phase of CS, which will be shown and discussed in this work.

In the following sections, two extensions are introduced that greatly improve so- 
lution speed when a dense constraint matrix provides multiple similar candidates. Extension 1 amalgamates the two loops in Chinneck's methods into a single one that both assesses the candidates and removes multiple constraints at each iteration. Extension 2 provides an early exit under certain conditions. The newly developed extensions can be combined with any of the state-of-the-art algorithms to increase their speed while improving/preserving the solution quality. For instance, the combination of Algorithm 2( $\infty)$ (explained in Section 2.1.4.2) and Extension 1 creates Algorithm $2 \mathrm{E} 1$.

\subsubsection{Extension 1 (E1): Multiple Simultaneous Removals}

There are three ways to assemble the candidate list in Algorithms 1-3. Regardless of the type of the list, previous research shows that the order in the sorted list of candidates is important: those earlier in the list are much more likely to be part of the MIN ULR than those later in the list. Thus, if multiple constraints at each iteration of a single loop are to be removed, it makes sense to select them from the start of the sorted list. This argument is especially potent when the constraint matrix is dense. In a dense constraint matrix, all constraints involve all of the variables, so all of the constraints are similar in structure. A consequence is that many of the constraints have similar impacts on objective function $Z$. For example, in the classification application, where the constraints are derived from the data points, data points that are close together generate similar constraints. These similar constraints have similar ranking scores in the candidate list, and hence have similar effects on $Z$ when removed from the LP, leading to this Observation: When candidates have similar ranking measures as the top-ranked element in the candidate list, they are likely to appear in the final MIN ULR set.

This observation allows both (1) the elimination of the inner loop to test individual 
candidate constraints and (2) the simultaneous removal of multiple candidates in the single remaining loop. The question is how to determine which ranking scores are "similar" to the top score. Candidates are sorted by descending ranking score and then standard methods for detecting abrupt changes in the mean of subsets of the set [46] are applied. All candidates up to the first identified abrupt change are selected for removal.

\subsubsection{Extension 2 (E2): Early Exit}

Extension 2 provides an early exit. If the number of candidates is less than or equal to $\ell$, then permanently remove all candidates and exit the algorithm. This is designated $\mathrm{E} 2(\ell)$

\subsection{Proof of Concept}

The newly developed extensions can be combined with any of the state-of-the-art MAX FS algorithms to increase their speed while improving/preserving the solution quality. For illustrative purposes, the introduced extensions are combined with Method B (Section 2.1.4.4) for the recovery of sparse solutions to underdetermined systems of linear equations. The steps of the extended algorithms BE1, B(2)E2( $\ell$, and BE1E2 $(\ell)$ are explained in Fig. 4.1, Fig. 4.2, and Fig. 4.3, respectively.

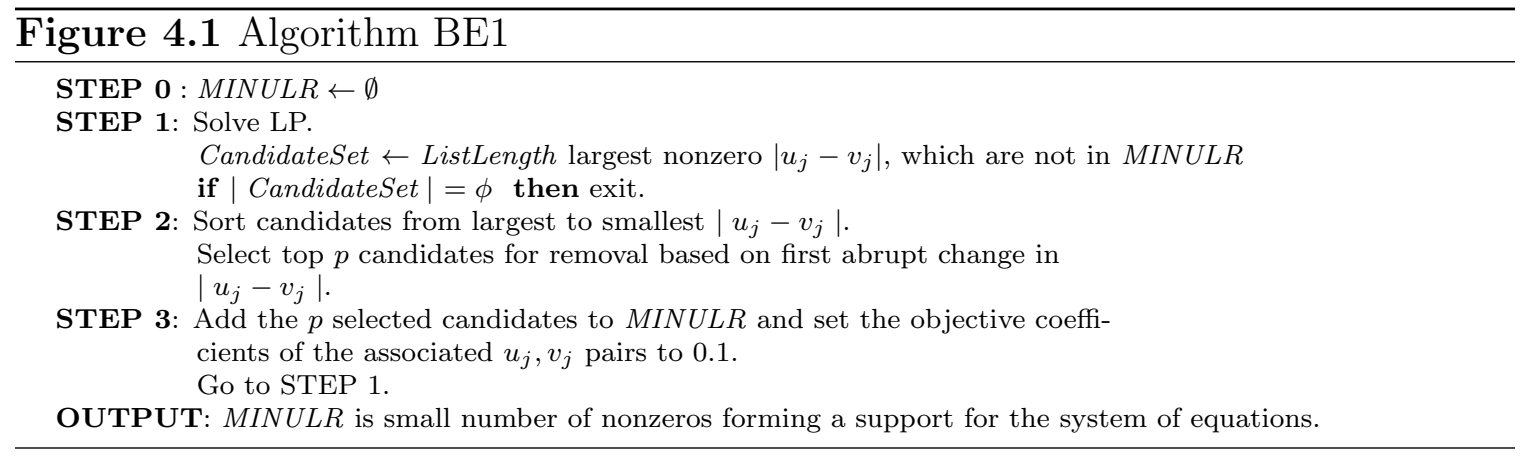




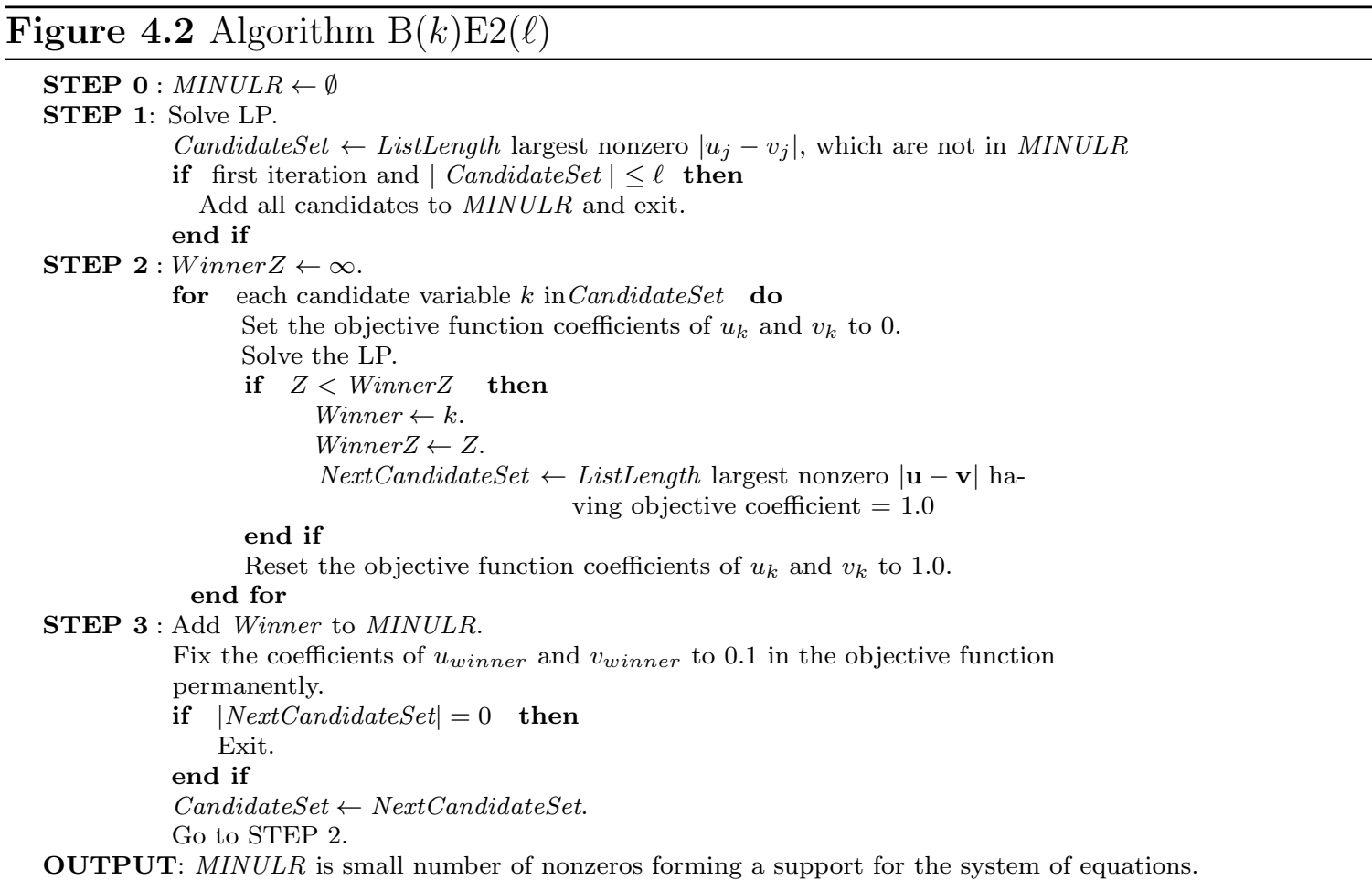

In the following sections, the extended algorithms are compared with the original Method B.

\subsubsection{Experimental Setup}

- Dataset: The test models are constructed based on [35] so that the solution is known in advance. The system is $\mathbf{A}_{128 \times 256} \mathbf{x}_{256 \times 1}=\mathbf{b}_{128 \times 1}$, providing a compression ratio $(C R)=\left(1-\frac{m}{n}\right) \times 100=50 \%$. A is a random matrix with values ranging uniformly from -10 to 10, and hence dense. The constructed input vector $\mathbf{x}$ is generated by randomly selecting $S$ columns of matrix $\mathbf{A}$ and assigning each selected column a weight chosen randomly from a normal distribution with mean 0 and standard deviation 1 . For each input sparsity $S, 100$ instances are constructed.

- Comparators: Algorithms BE1, B $(k) \mathrm{E} 2(\ell)$, and BE1E2 $(\ell)$ are compared with 
Figure 4.3 Algorithm BE1E2( $\ell)$

MINULR $\leftarrow \emptyset$

Set up the sparse recovery LP.

do:

Solve LP.

Construct CandidateSet: variables $x_{j}$ not in MINULR that have nonzero $\left|u_{j}-v_{j}\right|$.

if $\mid$ CandidateSet $\mid=\phi$ then exit.

if first iteration and $\mid$ CandidateSet $\mid \leq \ell$ then

Add all candidates to MINULR and exit.

end if

Sort candidates from largest to smallest $\left|u_{j}-v_{j}\right|$.

Select top $p$ candidates for removal based on first abrupt change in $\left|u_{j}-v_{j}\right|$.

Add the $p$ selected candidates to MINULR and set the objective coefficients of the

associated $u_{j}, v_{j}$ pairs to 0.1 .

end do

OUTPUT: MINULR is small number of nonzeros forming a support for the system of equations.

the original Methods $\mathrm{B}(k)$. The list length of $k=2(k$ is typically $1-7[11])$ is selected to provide fast solutions with good quality. Postprocessing is not used in any of these three methods.

\section{- Evaluation Metric:}

The signal processing literature shows that it is much more difficult to recover solutions having a higher fraction of nonzeros accurately, and hence the largest number of nonzeros in the input that an algorithm is able to recover accurately is an important measure of performance. This is known as the critical sparsity of a sparse recovery algorithm.

The Average Sparsity Error $\left(S_{e}\right)$ is also used in evaluating the performance of the MAX FS methods in sparse recovery. Sparse recovery is considered successful if $T$, the number of nonzeros in the solution, is identical to $S$, the number of nonzeros in the input (when $T=S$ both the sparsity level and the nonzero positions are identical in the input and estimated solutions). The average $T$-sparsity of the estimated solutions over 100 trials is, $T_{\text {average. }}$ The Average Sparsity Error (\%) of the average T-sparsity (Eqn. 4.1) shows the 
performance of each method in sparsity estimation. $S_{e}=0$ is desired.

$$
S_{e}=\frac{T_{\text {average }}-S}{S} \times 100
$$

- Software and Hardware: All algorithms are implemented in Matlab version 2020. The linear programming solver is MOSEK via the MOSEK Optimization Toolbox for Matlab version 8.1.0.56 [47]. Abrupt changes in the ranking scores in the candidate list are identified using the Matlab ischange() function. TIC and TOC command in Matlab is used to measure processing time of algorithms. The computations are carried out on a $3.40 \mathrm{GHz}$ Intel core $i 7$ machine with 16.0 GB RAM, running Windows 10.

\subsubsection{Results and Discussion}

Table 4.1 compares algorithms BE1, B $(k) \mathrm{E} 2(\ell)$, and BE1E2 $(\ell)$ with existing Method $\mathrm{B}(2)$. Results in the table are averages over 100 instances at each sparsity level $S$. To better generalize the results, the input sparsity $S$ is also shown in percentage $(s p \%)$ in parentheses with respect to the input length of $n=256$. For instance, the sparsity level of the first and the last row of the table are $\frac{12}{256} \times 100=4.69 \%$ and $\frac{96}{256} \times 100=37.50 \%$, respectively. The remaining columns present the average sparsity error in percent $S_{e}$ over 100 instances. The number of correct results is shown in parentheses. Results that are accurate in all 100 cases are bolded.

The last two rows in Table 4.1 have the following meanings: "Critical Sparsity" shows the maximum sparsity level that an algorithm is able to successfully match the input sparsity in all 100 cases, and "\# Success" expresses the total number of successes over each column. Extended algorithms B(2)E2( $\ell$ ) and BE1E2 $(\ell)$ have the same critical sparsity as the original Method $\mathrm{B}(2)$ and they provide the smallest 
critical sparsity. Extended Algorithm B $(2)$ E2 $(\ell)$ provides a larger number of successes than Method B(2) and the two other extended algorithms.

Table 4.1: Comparing the performance of Method B, Algorithm BE1, Algorithm $\mathrm{B}(2) \mathrm{E} 2(\ell)$, and Algorithm BE1E2 $(\ell)$ using the average sparsity error (\%) in sparse recovery ( $m=128, n=256$, and results are averaged over 100 random matrices).

\begin{tabular}{|c|c|c|c|c|}
\hline \multirow{2}{*}{$\mathbf{S}(s p \%)$} & \multicolumn{4}{|c|}{$S_{e}$} \\
\hline & Method B (2) & BE1 & $\mathbf{B}(\mathbf{2}) \mathbf{E 2}(\ell)$ & BE1E2 $(\ell)$ \\
\hline $12(4.69 \%)$ & $\mathbf{0}^{(100)}$ & $\mathbf{0}^{(100)}$ & $\mathbf{0}^{(100)}$ & $\mathbf{0}^{(100)}$ \\
\hline $16(6.25 \%)$ & $\mathbf{0}^{(100)}$ & $\mathbf{0}^{(100)}$ & $\mathbf{0}^{(100)}$ & $\mathbf{0}^{(100)}$ \\
\hline $20(7.81 \%)$ & $\mathbf{0}^{(100)}$ & $\mathbf{0}^{(100)}$ & $\mathbf{0}^{(100)}$ & $\mathbf{0}^{(100)}$ \\
\hline $24(9.37 \%)$ & $\mathbf{0}^{(100)}$ & $\mathbf{0}^{(100)}$ & $\mathbf{0}^{(100)}$ & $\mathbf{0}^{(100)}$ \\
\hline $28(10.94 \%)$ & $\mathbf{0}^{(100)}$ & $\mathbf{0}^{(100)}$ & $\mathbf{0}^{(100)}$ & $\mathbf{0}^{(100)}$ \\
\hline $32(12.50 \%)$ & $\mathbf{0}^{(100)}$ & $\mathbf{0}^{(100)}$ & $\mathbf{0}^{(100)}$ & $\mathbf{0}^{(100)}$ \\
\hline $36(14.06 \%)$ & $\mathbf{0}^{(100)}$ & $\mathbf{0}^{(100)}$ & $\mathbf{0}^{(100)}$ & $\mathbf{0}^{(100)}$ \\
\hline $40(15.62 \%)$ & $\mathbf{0}^{(100)}$ & $\mathbf{0}^{(100)}$ & $\mathbf{0}^{(100)}$ & $\mathbf{0}^{(100)}$ \\
\hline $44(17.18 \%)$ & $\mathbf{0}^{(100)}$ & $\mathbf{0}^{(100)}$ & $\mathbf{0}^{(100)}$ & $\mathbf{0}^{(100)}$ \\
\hline $48(18.75 \%)$ & $\mathbf{0}^{(100)}$ & $0.19^{(92)}$ & $\mathbf{0}^{(100)}$ & $\mathbf{0}^{(100)}$ \\
\hline $52(20.31 \%)$ & $\mathbf{0}^{(100)}$ & $0.49^{(86)}$ & $\mathbf{0}^{(100)}$ & $\mathbf{0}^{(100)}$ \\
\hline $56(21.87 \%)$ & $0.03^{(98)}$ & $2.49^{(60)}$ & $0.06^{(99)}$ & $0.17^{(98)}$ \\
\hline $60(23.44 \%)$ & $4.24^{(86)}$ & $10.61^{(57)}$ & $4.24^{(90)}$ & $10.72^{(86)}$ \\
\hline $64(25.00 \%)$ & $12.30^{(67)}$ & $19.40^{(28)}$ & $12.30^{(73)}$ & $20.31^{(73)}$ \\
\hline $68(26.56 \%)$ & $26.53^{(45)}$ & $37.55^{(8)}$ & $26.53^{(47)}$ & $36.69^{(39)}$ \\
\hline $72(28.12 \%)$ & $34.87^{(25)}$ & $45.98^{(1)}$ & $34.87^{(30)}$ & $48.29^{(19)}$ \\
\hline $76(29.69 \%)$ & $45.85^{(5)}$ & $49.07^{(1)}$ & $45.85^{(7)}$ & $59.08^{(6)}$ \\
\hline $80(31.25 \%)$ & $46.52^{(4)}$ & $46.71^{(0)}$ & $46.52^{(3)}$ & $56.59^{(3)}$ \\
\hline $84(32.81 \%)$ & $43.55^{(0)}$ & $44.02^{(0)}$ & $43.55^{(1)}$ & $51.36^{(0)}$ \\
\hline $88(34.37 \%)$ & $39.98^{(0)}$ & $40.02^{(0)}$ & $39.98^{(0)}$ & $45.45^{(0)}$ \\
\hline $92(35.94 \%)$ & $36.00^{(0)}$ & $36.02^{(0)}$ & $36.00^{(0)}$ & $39.13^{(0)}$ \\
\hline $96(37.50 \%)$ & $31.96^{(0)}$ & $32.01^{(0)}$ & $31.96^{(0)}$ & $33.33^{(0)}$ \\
\hline Critical Sparsity & $52(20.31 \%)$ & $44(17.18 \%)$ & $52(20.31 \%)$ & $52(20.31 \%)$ \\
\hline \# Success & 1430 & 1233 & 1450 & 1424 \\
\hline
\end{tabular}

Table 4.2 shows the number of LPs solved ("LPs"), and the processing time in seconds ("Sec") for algorithms BE1, B(2)E2( $\ell), \mathrm{BE} 1 \mathrm{E} 2(\ell)$, and Method B(2). The smallest number of LPs and time in seconds are bolded. Algorithm BE1E2 $(\ell)$ requires the fewest LP solutions at all values of $S$ when compared against Method B(2) and the two other extended algorithms. Table 4.3 reports the difference of the required LPs and processing time of each method from the best value of LPs and Sec for each sparsity level. The last three rows give additional metrics: the largest difference from the best LPs and Sec, the average difference from the best LPs and Sec, and number of best LPs and Sec for each algorithm. Based on the results, the extended algorithms 
are significantly faster than the original Method $\mathrm{B}(2)$. Algorithm BE1E2 $(\ell)$ is the fastest method, providing: (1) the smallest average difference from best LPs, (2) the smallest average difference from best Sec, and (3) the smallest (best) number of LPs and Sec at all sparsity levels versus 0, 9 and 7 for Method B(2), Algorithm $\mathrm{BE} 1$, and Algorithm $\mathrm{B}(2) \mathrm{E} 2(\ell)$, respectively. Method $\mathrm{B}(2)$ requires the most LPs and processing time.

Table 4.2: Number of LPs and processing times for Algorithm BE1, Algorithm $\mathrm{B}(2) \mathrm{E} 2(\ell)$, and Algorithm BE1E2( $\ell)$ vs. Method $\mathrm{B}(2)$ in sparse recovery $(m=128$, $n=256$, and results are averaged over 100 random matrices).

\begin{tabular}{|c|c|c|c|c|c|c|c|c|}
\hline \multirow{2}{*}{$\mathbf{S}(s p \%)$} & \multicolumn{2}{|c|}{ Method B(2) } & \multicolumn{2}{|c|}{ BE1 } & \multicolumn{2}{|c|}{$\mathbf{B}(2) \mathbf{E 2}(\ell)$} & \multicolumn{2}{|c|}{$\operatorname{BE1E2}(\ell)$} \\
\hline & LPs & Sec & LPs & Sec & LPs & Sec & LPs & Sec \\
\hline $12(4.69 \%)$ & 23 & 0.84 & 2.07 & 0.31 & 1 & 0.06 & 1 & 0.06 \\
\hline $16(6.25 \%)$ & 31 & 1.16 & 2.27 & .24 & 1 & 0.05 & 1 & 0.05 \\
\hline $20(7.81 \%)$ & 39 & 1.72 & 2.13 & 0.23 & 1 & 0.06 & 1 & 0.06 \\
\hline $24(9.37 \%)$ & 47 & .30 & 2.23 & 0.24 & 1 & 0.06 & $\mathbf{1}$ & 0.06 \\
\hline $28(10.94 \%)$ & 55 & .98 & 2.28 & 0.26 & 1 & .06 & 1 & 0.06 \\
\hline $32(1$ & 63 & 3.02 & 2.3 & 0.27 & 1 & 0.07 & 1 & 0.07 \\
\hline $36(14.06 \%)$ & 71 & 3.66 & 2.29 & 0.28 & 1 & 0.07 & 1 & 0.07 \\
\hline $40(15.62 \%)$ & 79 & 4.27 & 2.19 & 0.27 & 2.19 & 0.27 & 1.03 & 0.08 \\
\hline $44(17.18 \%)$ & 87 & 4.27 & 2.17 & 0.27 & 12.18 & 0.69 & 1.20 & 0.12 \\
\hline $48(18.75 \%)$ & 95 & 4.60 & 2.37 & 0.33 & 31.08 & 1.74 & 1.49 & 0.23 \\
\hline $52(20.31 \%)$ & 103 & 5.33 & 2.69 & 0.41 & 77.50 & 4.35 & 2.35 & 0.40 \\
\hline $56(21.87 \%)$ & 111.12 & 5.99 & 3.16 & 0.75 & 100.12 & 5.77 & 3.01 & 0.53 \\
\hline $60(23.44 \%)$ & 127.68 & 7.02 & 3.90 & 0.88 & 124.14 & 7.28 & 3.82 & 0.73 \\
\hline $64(25.00 \%)$ & 152.26 & 8.56 & 4.63 & 0.90 & 152.26 & 9.09 & 4.63 & 0.90 \\
\hline $68(26.56 \%)$ & 189.39 & 10.72 & 5.46 & 1.12 & 189.39 & 11.34 & 5.46 & 1.12 \\
\hline $72(28.12 \%)$ & 214.48 & 12.15 & 5.95 & 1.31 & 214.48 & 12.86 & 5.95 & 1.31 \\
\hline $76(29.69 \%)$ & 245.06 & 13.91 & 6.36 & 1.42 & 245.06 & 14.53 & 6.36 & 1.42 \\
\hline $80(31.25 \%)$ & 245.06 & 14.37 & 6.18 & 1.41 & 254.84 & 14.53 & 6.18 & 1.41 \\
\hline $84(32.81 \%)$ & 254.84 & 14.50 & 6.85 & 1.58 & 257.09 & 14.50 & 6.85 & 1.58 \\
\hline $88(34.37 \%)$ & 257.09 & 14.55 & 6.75 & 1.57 & 258.20 & 14.69 & 6.75 & 1.57 \\
\hline 92( & 258.18 & 14.83 & 6.87 & 1.67 & 258.18 & 14.38 & 6.87 & 1.67 \\
\hline $96(37.50 \%)$ & 257.94 & 14.64 & 7.30 & 1.79 & 257.97 & 14.36 & 7.30 & 1.79 \\
\hline
\end{tabular}

The new MAX FS algorithms for dense constraint matrices are presented in Chapter 5 and Chapter 6 . 
Table 4.3: Differences from the smallest LPs and processing time (the results averaged over 100 random matrices).

\begin{tabular}{|c|c|c|c|c|c|c|c|c|}
\hline \multirow{2}{*}{$\mathbf{S}(s p \%)$} & \multicolumn{2}{|c|}{$\mathrm{B}(2)$} & \multicolumn{2}{|c|}{ BE1 } & \multicolumn{2}{|c|}{$\mathbf{B}(2) \mathbf{E} 2(\ell)$} & \multicolumn{2}{|c|}{ BE1E2 $(\ell)$} \\
\hline & LPs & Sec & LPs & Sec & LPs & Sec & LPs & Sec \\
\hline $12(4.69 \%)$ & 22 & 0.78 & 1.07 & 0.252 & $\mathbf{0}$ & $\mathbf{0}$ & $\mathbf{0}$ & 0 \\
\hline $16(6.25 \%)$ & 30 & 1.11 & 1.27 & 0.19 & $\mathbf{0}$ & $\mathbf{0}$ & $\mathbf{0}$ & $\mathbf{0}$ \\
\hline $20(7.81 \%)$ & 38 & 1.66 & 1.13 & 0.17 & 0 & 0 & $\mathbf{0}$ & 0 \\
\hline $24(9.37 \%)$ & 46 & 2.24 & 1.23 & 0.18 & $\mathbf{0}$ & $\mathbf{0}$ & 0 & 0 \\
\hline $28(10.94 \%)$ & 54 & 2.92 & 1.28 & 0.20 & $\mathbf{0}$ & $\mathbf{0}$ & $\mathbf{0}$ & $\mathbf{0}$ \\
\hline $32(12.50 \%)$ & 62 & 2.95 & 1.30 & 0.20 & $\mathbf{0}$ & $\mathbf{0}$ & $\mathbf{0}$ & 0 \\
\hline $36(14.06 \%)$ & 70 & 3.59 & 1.29 & 0.21 & $\mathbf{0}$ & $\mathbf{0}$ & $\mathbf{0}$ & $\mathbf{0}$ \\
\hline $40(15.62 \%)$ & 77.97 & 4.19 & 1.16 & 0.19 & 1.16 & 0.19 & $\mathbf{0}$ & 0 \\
\hline $44(17.18 \%)$ & 85.80 & 4.15 & 0.97 & 0.15 & 10.98 & 0.57 & $\mathbf{0}$ & $\mathbf{0}$ \\
\hline $48(18.75 \%)$ & 93.51 & 4.37 & 0.88 & 0.10 & 29.59 & 1.51 & 0 & $\mathbf{0}$ \\
\hline $52(20.31 \%)$ & 100.65 & 4.93 & 0.34 & 0.01 & 75.15 & 3.95 & $\mathbf{0}$ & 0 \\
\hline $56(21.87 \%)$ & 108.11 & 5.46 & 0.15 & 0.22 & 97.11 & 5.24 & $\mathbf{0}$ & $\mathbf{0}$ \\
\hline $60(23.44 \%)$ & 123.86 & 6.29 & 0.08 & 0.15 & 120.32 & 6.55 & $\mathbf{0}$ & $\mathbf{0}$ \\
\hline $64(25.00 \%)$ & 147.63 & 7.66 & $\mathbf{0}$ & $\mathbf{0}$ & 147.63 & 8.19 & $\mathbf{0}$ & 0 \\
\hline $68(26.56 \%)$ & 183.93 & 9.60 & $\mathbf{0}$ & 0 & 183.93 & 10.22 & $\mathbf{0}$ & $\mathbf{0}$ \\
\hline $72(28.12 \%)$ & 208.53 & 10.84 & $\mathbf{0}$ & 0 & 208.53 & 11.55 & $\mathbf{0}$ & $\mathbf{0}$ \\
\hline $76(29.69 \%)$ & 238.7 & 12.49 & 0 & 0 & 238.7 & 13.11 & 0 & $\mathbf{0}$ \\
\hline $80(31.25 \%)$ & 238.88 & 12.96 & $\mathbf{0}$ & 0 & 248.66 & 13.12 & 0 & 0 \\
\hline $84(32.81 \%)$ & 247.99 & 12.92 & 0 & 0 & 250.24 & 12.92 & 0 & $\mathbf{0}$ \\
\hline $88(34.37 \%)$ & 250.34 & 12.98 & $\mathbf{0}$ & $\mathbf{0}$ & 251.45 & 13.12 & $\mathbf{0}$ & 0 \\
\hline $92(35.94 \%)$ & 251.31 & 13.16 & $\mathbf{0}$ & $\mathbf{0}$ & 251.31 & 12.71 & $\mathbf{0}$ & 0 \\
\hline $96(37.50 \%)$ & 250.64 & 12.85 & 0 & 0 & 250.67 & 12.57 & $\mathbf{0}$ & 0 \\
\hline Largest Difference & 251.31 & 13.16 & 1.30 & 0.25 & 251.45 & 13.12 & $\mathbf{0}$ & $\mathbf{0}$ \\
\hline Average Difference & 133.175 & 6.82 & 0.55 & 0.10 & 107.52 & 5.705455 & $\mathbf{0}$ & $\mathbf{0}$ \\
\hline \# of bests & 0 & 0 & 9 & 9 & 7 & 7 & 22 & 22 \\
\hline
\end{tabular}




\section{Chapter 5}

\section{Extended MAX FS for Binary Classification}

In the current information age due to digitalization, the amount of data that is being generated is growing in a faster exponential rate than Moore's law. According to the International Data Corporation (IDC), worldwide data generation will grow to 175 zettabytes by 2025 [48. This has lead to a growing interest in the development of Machine Learning (ML) algorithms for data processing and extract valuable insights from databases. In [49], the National Cancer Institute (NCI) reported the significance role of ML in cancer care to improve the diagnosis speed, accuracy, better health outcome, etc. Moreover, NCI is investing more in supporting ML technology. According to [50], the global machine learning market is expected to reach $\$ 20.83 \mathrm{~B}$ in 2024 , more than thirteen times the $\$ 1.58 \mathrm{~B}$ that was valued in 2017 . In fact, ML techniques are the foundation of a broad spectrum of next-generation technologies. Thus, it has lead to a massive interest in learning and developing ML techniques. According to an institution survey of a radiology department [51], 97\% of their residents and fellows were willing to apply or train ML models to do some of the tasks of a radiologist. 
As a result, engineers has been motivated to work closely with medical professionals to design and develop ML algorithms that can be implemented into clinical practice. Therefore, researchers need to help ML to continue its momentum to develop and mature to satisfy the need of health care.

Depending on the type of data and tasks at hand, different ML algorithms, also called models, can be employed. One central model in machine learning is classification since it is an important tool for intelligent decision making such as in areas like medicine [52], government [53], and economics [54]. Classification is a data analysis technique that is used to discover patterns to categorize the data into classes. Classification may be divided into the following four groups: binary classification, multi-class classification, multi-label classification, and imbalanced classification.

Although the proposed method can also be used for muti-class classification, this chapter focuses on binary classification, which refers to classifying the components of a given dataset into two classes. In Section 5.1 , the literature widely used binary classification models are briefly explained. Then, Section 5.2 details a newly developed MAX FS method based on Extension 1 (refer to 4.1.1). In Section 5.3, the performance of the new extended MAX FS algorithm is evaluated in two sets of experiments to show: (1) higher performance of the new method compared to the state-of-the-art MAX FS methods in terms of speed and quality, and (2) superior performance of the extended MAX FS method compared to the widely used binary classification methods. The following section provides a background in binary classification problem and its well-known algorithms. 


\subsection{Existing Binary Classification Algorithms}

Binary classification is a supervised learning technique, which refers to a process of mapping each given instance to a label of one of two classes using a machine learning algorithm (binary classification model). A wide variety of binary classification models have been proposed and compared in the literature [55, 56]. The performance of models depends on the dataset and the classification problem. Therefore, different classification models are employed and their performance is evaluated on the given task. Then, a model with a higher performance is selected for the problem at hand. In general, some widely used binary classification models are K-Nearest Neighbours (KNN) [57, Support Vector Machines (SVM) [58], Naive Bayes (NB) [59], and Logistic Regression (LR) [60]. MAX FS algorithms can be applied for binary classification. In [11, Chinneck showed the superior performance of MAX FS algorithms (Algorithm 1 and Algorithm 2) for binary classification (see Chapter 2 for details). The wellknown non-MAX FS classification models and their characteristics are summarized below.

K-Nearest Neighbours (KNN): KNN classifiers are an example of what is called instance-based or memory-based supervised learning, which classify by memorizing the labeled examples that are available in the training set and then using those memorized examples to classify new objects later. The $K$ in KNN refers to the number of nearest neighbors the classifier retrieves and uses in order to make its prediction. Smaller $K$ may increase the risk of overfitting as only local changes are captured. Good generalization cannot be expected for unseen data when $K$ is small. Therefore, the choice of $K$ is crucial in KNN classifier.

In training, both features and labels of the training set are saved in memory. For the test data, the following steps are considered in KNN: 
1. Determine the parameter $K$.

2. For each point in the test set, calculate distances from the points in train set.

3. Find $K$ closest points for each test points.

4. Conduct majority vote to predict the class label.

In the KNN algorithm, four things need to be specified: (1) a distance metric (e.g. typically Euclidean distance), (2) number of nearest neighbors (e.g. $K=5),(3)$ optional weighting function on the neighbor points, and (4) a method for aggregating the classes of neighbor points (e.g. simple majority vote or class with the most representatives among nearest neighbors). Some advantages and disadvantages of the KNN classfier are 61]:

- Advantages:

- The classifier has a very simple implementation.

- Training is fast.

- The classifier is robust with regard to the search space (classes do not have to be linearly separable).

- Disadvantages:

- Memory requirements for the algorithm is high.

- Classification results are dependent on the choice of $K$ (a small value of $K$ could lead to overfitting as well as a big value of $K$ can lead to underfitting).

- It needs parameter tuning.

- Accuracy depends on the quality of the data and irrelevant attributes may lead to poor performance. 
Support Vector Machines (SVM): One way to define a good classifier is to reward classifiers for the amount of separation that they can provide between the two classes since this provides better generalization. To do this, the concept of classifier margin is defined. The margin is the perpendicular distance from the decision boundary to the nearest data point.

In other words, the margin is the width that the decision boundary can be increased before hitting a data point. Among all possible classifiers that separate two classes, the best classifier is the one that has the maximum amount of margin. The maximum margin classifier is called the Support Vector Machine (SVM) (also known as an Lagrangian Support Vector Machine (LSVM) for a support vector machine with linear kernel).

A regularization parameter, $C$, is used in SVM to adjust the interplay between margin and the misclassification rate. Larger values of $C$ represent less regularization and cause the model to fit the training set with smaller errors On the other hand, very small values of $\mathrm{C}$ use more regularization and encourage the classifier to find a large margin on the decision boundary, even if that decision boundary leads to higher misclassification rate. Some advantages and disadvantages of the SVM model are $[55,62]$ :

- Advantages:

- SVM does not overfit.

- Several kernels are available for use with SVM for dataset that contain nonlinearities.

- SVM has a good generalization ability.

- Disadvantages: 
- Training of SVM is slow.

- Choice of parameters plays a significant role in performance of SVM.

- SVM requires normalization of input data and parameter tuning.

- In SVM, it is hard to interpret why a prediction was made (not explainable).

Naive Bayes (NB): A Naive Bayes classifier is a statistical classification technique based on Bayes' theorem 63. Naive Bayes shows the relationship between the independent samples and the target variable. Consider a feature set $X=\left\{x_{1}, x_{2}, \ldots, x_{n}\right\}$ and a target variable $y$, then the objective function of Naive Bayes is:

$$
\hat{y}=\operatorname{argmax}_{y} P(y) \prod_{i=1}^{n} P\left(x_{i} \mid y\right),
$$

where $P(y)$ and $P\left(x_{i} \mid y\right)$ are the probability of a value $y$ and the posterior probabilities of $x_{i}$ given the values of $y$. One of the main types of NB is Gaussian NB (moving forward, the term NB refers to Gaussian NB). To calculate the mean value $\mu_{y}$ and the variance $\sigma_{y}^{2}$, the likelihood of features in NB is obtained as follows 64]:

$$
P\left(x_{i} \mid y\right)=\frac{1}{\sqrt{2 \pi \sigma_{y}^{2}}} \exp \left(-\frac{\left(x_{i}-\mu_{y}\right)^{2}}{2 \sigma_{y}^{2}}\right) .
$$

Some advantages and disadvantages of Naive Bayes model are [62]:

- Advantages:

- Training of Naive Bayes classifier is fast.

- A Naive Bayes classifier is not sensitive to irrelevant features.

- Disadvantages: 
- Naive Bayes assumes that all features are independent, which is a rare case in real-world datasets.

- The classifier assigns zero probability to a categorical variable of a category, which has not been presented in the training dataset.

Logistic Regression (LR): Logistic Regression (LR) is a widely used linear classifier model. The logistic regression model computes a weighted sum of the input features $x_{i}$ and the intercept term $\hat{b}$, but it runs this result through the non-linear logistic function, shown in Eq. 5.3. The weight vector is $\hat{w}=\left(\hat{w}_{1}, \ldots, \hat{w}_{n}\right)$. The effect of applying the logistic function is to compress the output of the linear function so that it is limited to a range between 0 and 1 . This output probability is snapped to a binary class value, with 0.5 as the decision boundary. To illustrate, for points with a probability greater or equal to 0.5 on the logistic function, $\hat{y}=1$, and $\hat{y}=0$ is returned for the points having probability less than 0.5 on the logistic function.

$$
\hat{y}=\operatorname{logistic}\left(\hat{b}+\hat{w}_{1} x_{1}+\ldots+\hat{w}_{n} x_{n}\right)=\frac{1}{1+\exp \left[-\left(\hat{b}+\hat{w}_{1} x_{1}+\ldots+\hat{w}_{n} x_{n}\right)\right]} .
$$

A regularization penalty on the model coefficients can also be applied with logistic regression, and is controlled with the parameter $C$. With large values of $C$, logistic regression tries to fit the training data where as for small values of $C$, the method tries harder to find coefficients that are closer to 0 , even if that model fits the training data a little bit worse. Some advantages and disadvantages of LR model are [55, 56]:

- Advantages:

- Its predictions are easy to interpret.

- It has good performance over big data.

- Disadvantages: 
- LR requires large training dataset to provide consistent classification.

- Logistic regression may overfit in high dimensional datasets.

MAX FS can be used for the classification problem [11, 14, 28, 42]. However, MAX FS algorithms are slow. In this chapter, the MAX FS algorithms are modified to make them faster. In the following sections, an extended MAX FS binary classification algorithm to improve the speed of the original state-of-the-art MAX FS binary classification algorithm is detailed. Its performance is evaluated and compared with the original MAX FS algorithms and the mentioned well-known binary classification models.

\subsection{Extended MAX FS Binary Classification Al- gorithm}

Prior work shows that Algorithm 2 provides a major speed-up over Algorithm 1 for binary classification [11], with a tiny loss in average accuracy. In this section, an extension of Algorithm 2 introduced in Section 2.1.4.2 is used to increase the speed of the original MAX FS methods with no loss of accuracy. New Algorithm 2E1 is summarized in Fig. 5.1. In Algorithm 2E1, the inner loop of Algorithm 2 is eliminated, and multiple candidates are added to the output in the remaining single loop. This is significantly faster than the existing Algorithm 2.

A standard elastic program is used because the variables are unrestricted. As explained in [11], violated constraints correspond to misclassified points, so the product ranking score (Eqn. 2.3) used in Algorithm 2 estimates the drop in $Z$ relatively accurately. Since all attribute values are specified for all data points, the constraint matrix $\mathbf{D}$ is dense, so Extension 1 is applied. To illustrate the workings of Exten- 
sion 1, Fig. 5.2 shows the first sorted candidate list for the "Ozone Level Detection" dataset available in UCI repository [65]. The first sharp drop-off occurs after the fifth candidate, so the first five constraints/points are removed in this case.
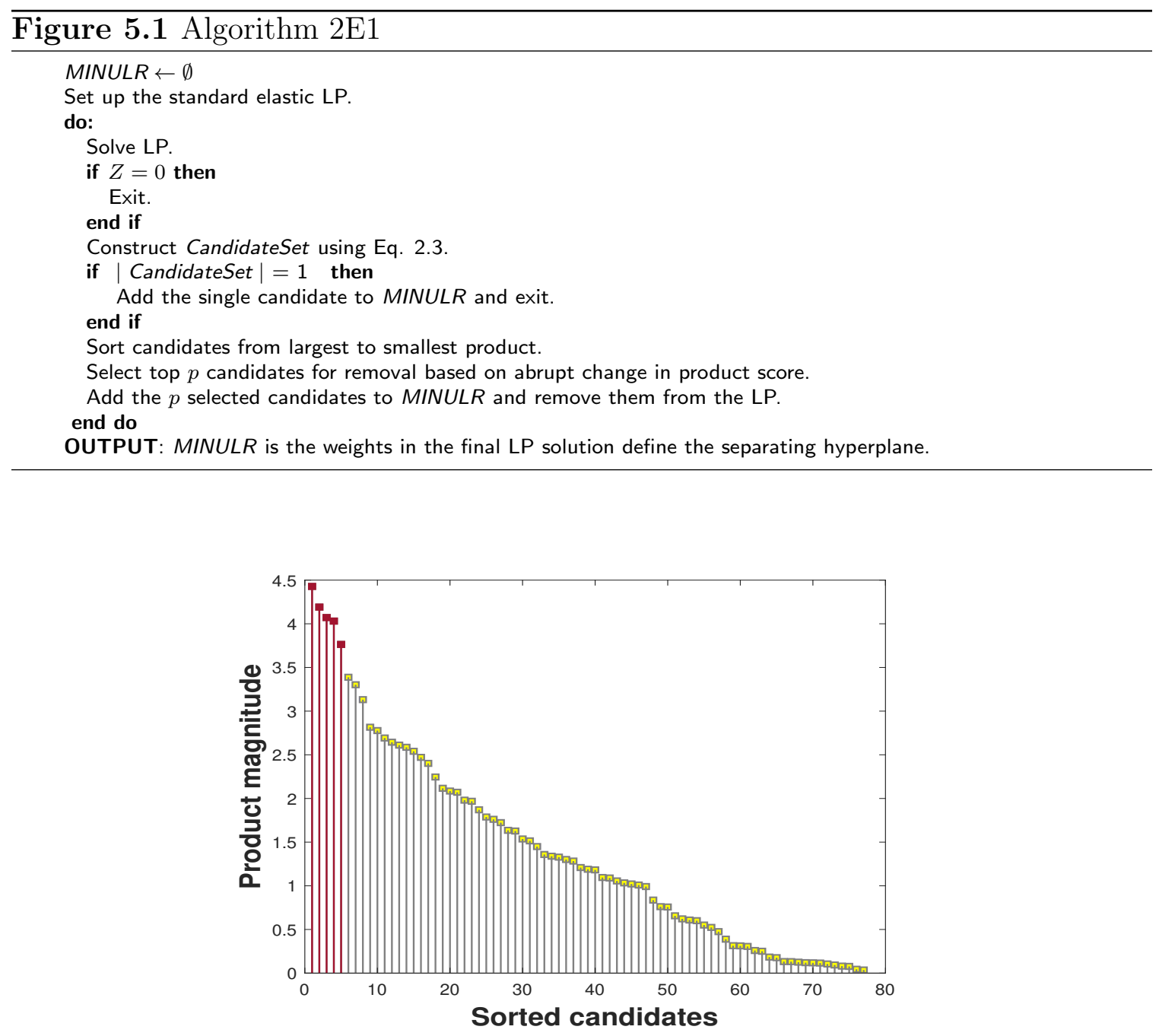

Figure 5.2: First sorted candidate list for "Ozone Level Detection" dataset.

The following Section 5.3 and Section 5.4 show the superior performance of Algorithm 2E1 when applied on different datasets as compared with MAX FS and non MAX FS binary models. 


\subsection{Experimental Setup}

Two sets of experiments compare the performance of Algorithm 2E1 with: (i) the state-of-the-art MAX FS algorithms (Algorithm 2( $\infty$ ) and Algorithm 2(1)) to show the higher speed of Algorithm 2E1 with no loss in accuracy (Scenario I), and (ii) the four mentioned non MAX FS methods to show the superior performance of Algorithm 2E1 compared to widely used binary classification models under 10-fold cross-validation (Scenario II). Both scenarios focus on placing a single separating hyperplane. The procedure for solving classification problems in Scenario II can be summarised as follows:

- Identify and handle the missing values.

- Employ random over-sampling technique to handle imbalanced classes.

- Select a binary classification model.

- Use 10-fold cross-validation to measure the performance of the classification algorithms (the same folds are used throughout the implementation for all models to ensure the uniformity).

- Evaluate the performance of the classification methods based on different metrics.

Details follow below.

- Comparators: In Scenario I, Algorithm 2E1 is compared with two existing algorithms: (i) original Algorithm 2( $\infty$ ) and (ii) original Algorithm 2(1). Both have been shown to provide high accuracies in binary classification [11]. A recent study [56] reported KNN, LR, and Bayes model as widely used and fast 
binary classification models for single separation. SVM also performs well in binary classification [66.

In Scenario II, Algorithm 2E1 will be compared against the four well-known methods. Hyperparameter tuning is required to obtain the best performance for some classifiers. For the methods that require hyperparameter tuning, two sets of results are reported: (1) default settings without hyperparameter tuning "Def", and (2) with hyperparameter tuning "Tune" ("Def" applies if unspecified). A recent study, [67], provides a comprehensive overview of hyperparameter optimization of common ML models. Hyperparameters are tuned using the hyperparameter optimization methods and search ranges suggested in [67]. There is no need to tune hyperparameters in NB and LR since the performance of these models mainly depends on how well the datasets follow the Gaussian distribution and have small correlation of independent features, respectively [67]. Table 5.1 summarizes the hyperparameters "HP", selected hyperparameter optimization methods "HPO", and search range for the KNN and SVM classifiers.

Table 5.1: Configuration space for the hyperparameters and methods of the comparators.

\begin{tabular}{|c|c|c|c|}
\hline ML Alg. & HP & HPO Method & Search range \\
\hline KNN & K & Hyperband 68 & {$[1,20]$} \\
\hline \multirow{2}{*}{ SVM } & C & \multirow{2}{*}{ BO-TPE 69] } & {$[0.1,50]$} \\
\cline { 2 - 2 } & kernel & & {$[$ 'linear', 'poly',' 'rbf', 'sigmoid'] } \\
\hline
\end{tabular}

- Evaluation Metrics: The following metrics are used to evaluate the performance of the newly developed methods and comparators.

- Accuracy: Accuracy is used to evaluate the quality of the solutions:

$$
\text { Accuracy }=\frac{\text { Total \# correctly classified instances }}{\text { Total \# instances }} .
$$


- Precision: It is useful when the cost of false positives is high. TP and FP refer to True Positive $(\mathrm{TP})$ and False Positive $(\mathrm{FP})$, respectively.

$$
\text { Precision }=\frac{T P}{T P+F P} .
$$

- Recall: Also known as true positive rate, Recall is important when the cost of false negatives $(\overrightarrow{F N})$ is high such as cancer detection, tumor detection, etc. Recall is obtained using Eqn (5.6).

$$
\text { Recall }=\frac{T P}{T P+F N} .
$$

- F1 score: This metric combines precision and recall into a single number.

$$
F 1=\frac{2 T P}{2 T P+F N+F P} .
$$

- ROC_AUC score: $\mathrm{ROC}$ (Receiver Operating Characteristics) is a probability curve and is plotted with true positive rate (y-axis) vs. the false positive rate (x-axis). AUC (Area Under the Curve) represents the degree of separability and shows how much a model is capable of distinguishing between classes. AUC near to 0 means a model has poor separability. A model has no class separation capacity when AUC is 0.5).

- Data Sets: Binary classification problems are derived from datasets in the UCI Repository of Machine Learning Databases 65. Table 5.2 and Table 5.3 summarize the characteristics of the datasets used in Scenario I and Scenario II, respectively. In the first scenario, the training set is identical to the entire dataset to compare with previous results [11]. In Scenario II, real-world medical 
and biological datasets for disease diagnosis are used. The task is to predict whether the patient has the disease (Class 1) or not (Class 0). The same folds are used throughout the implementation for all the models to ensure uniformity.

Table 5.2: Classification Datasets for Scenario I.

\begin{tabular}{|l||c|c|}
\hline \multicolumn{1}{|c|}{ Dataset } & Instances & Features \\
\hline \hline Breast Cancer Wisconsin Original & 683 & 9 \\
\hline Cardiotocography (type 1 vs. others) & 2126 & 34 \\
\hline Cardiotocography (type 2 vs. others) & 2126 & 34 \\
\hline Car Evaluation (type 1 vs. others) & 1728 & 6 \\
\hline Car Evaluation (type 2 vs. others) & 1728 & 6 \\
\hline Car Evaluation (type 3 vs. others) & 1728 & 6 \\
\hline Car Evaluation (type 4 vs. others) & 1728 & 6 \\
\hline Climate Model Simulation Crashes & 540 & 18 \\
\hline Credit Approval & 653 & 15 \\
\hline Diabetic Retinopathy Debrecen & 1151 & 19 \\
\hline Glass Identification & 214 & 9 \\
\hline Heart Disease & 297 & 13 \\
\hline Hepatitis Domain & 112 & 18 \\
\hline Ionosphere & 351 & 33 \\
\hline BUPA Liver Disorders & 341 & 6 \\
\hline Ozone Level Detection & 1848 & 102 \\
\hline Parkinson's Data Set & 195 & 22 \\
\hline Pima Indians Diabetes & 768 & 8 \\
\hline Thyroid Gland Data & 215 & 5 \\
\hline Vowel Recognition Data & 990 & 11 \\
\hline
\end{tabular}

Table 5.3: Classification Datasets Scenario II.

\begin{tabular}{|l||c|c|}
\hline \multicolumn{1}{|c||}{ Dataset } & Instances & Features \\
\hline \hline Breast Cancer Wisconsin & 569 & 31 \\
\hline Diabetic Retinopathy Debrecen & 1151 & 19 \\
\hline Heart Disease (HD) & 297 & 13 \\
\hline BUPA Liver Disorders & 341 & 6 \\
\hline Parkinson's Data Set & 195 & 22 \\
\hline Pima Indians Diabetes & 768 & 8 \\
\hline Hepatitis Domain & 112 & 18 \\
\hline
\end{tabular}

- Software and Hardware: Algorithm 2E1 and the original MAX FS algorithms are implemented in Matlab version 2020. The linear programming solver is MOSEK via the MOSEK Optimization Toolbox for Matlab version 8.1.0.56 47]. Abrupt changes in the ranking scores in the candidate list are identified using the Matlab ischange() function. The computations are carried 
out on a $3.40 \mathrm{GHz}$ Intel core $i 7$ machine with 16.0 GB RAM, running Windows 10. The non MAX FS comparators are implemented in Python 3 using scikit-learn library 70 .

\subsection{Results and Discussion}

\section{Scenario I}

The results in this section show the higher accuracy and greater speed of Algorithm 2E1 vs. original Algorithms 2( $\infty)$ and 2(1) for binary classification. Table 5.4 reports algorithm accuracies with the best results bolded. Table 5.5 shows the difference from the best accuracy on each data set. The last three rows give additional metrics: the largest difference from the best accuracy, the average difference from the best accuracy, and number of best accuracies obtained by each algorithm.

Algorithm 2E1 provides better total accuracy. It has the smallest average difference from best accuracy, and provides the best accuracy for 12 of the data sets while it is 8 for the two existing algorithms. The largest difference from best accuracy for Algorithm 2E1 is -1.38 , smaller than for the other two $(-4.28$ for both).

Table 5.6 compares the number of linear programs solved (LPs) and the processing time in seconds $(\mathrm{Sec})$ for each algorithm, with best results bolded. Algorithm 2E1 requires the fewest LP solutions and the least processing time in all cases. Table 5.7 reports the percentage reduction in number of LPs and processing time for Algorithm $2 \mathrm{E} 1$ vs. the comparators for each dataset. For example, the processing times for Algorithms $2(\infty)$ and $2 \mathrm{E} 1$ are 3.1 and 0.3 seconds for the first dataset in Table 5.6. respectively, so Algorithm 2E1 provides a 90.32\% reduction in processing time

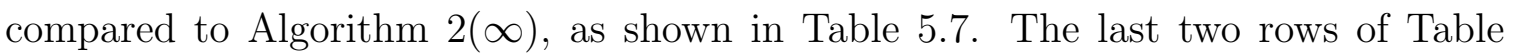
5.7 show the maximum and average speed improvements obtained by Algorithm 2E1 
Table 5.4: Algorithm Accuracies for Classification Datasets.

\begin{tabular}{|l|c|c|c|}
\hline \multirow{2}{*}{ Dataset } & \multicolumn{3}{|c|}{ Accuracy } \\
\cline { 2 - 4 } & Algorithm 2( $\infty)$ & Algorithm 2(1) & Algorithm 2E1 \\
\hline Breast Cancer Wisconsin Original & 98.10 & 98.10 & $\mathbf{9 8 . 3 9}$ \\
\hline Cardiotocography (type 1 vs.others) & 99.72 & 99.72 & $\mathbf{9 9 . 8 6}$ \\
\hline Cardiotocography (type 2 vs.others) & 99.67 & 99.58 & $\mathbf{9 9 . 8 1}$ \\
\hline Car Evaluation (type 1 vs.others) & 78.47 & 78.47 & $\mathbf{8 2 . 7 5}$ \\
\hline Car Evaluation (type 2 vs.others) & $\mathbf{9 6 . 0 1}$ & $\mathbf{9 6 . 0 1}$ & $\mathbf{9 6 . 0 1}$ \\
\hline Car Evaluation (type 3 vs.others) & 87.79 & 88.42 & $\mathbf{8 9 . 0 1}$ \\
\hline Car Evaluation (type 4 vs.others) & $\mathbf{9 8 . 1 5}$ & 98.09 & 98.09 \\
\hline Climate Model Simulation Crashes & $\mathbf{9 8 . 8 9}$ & 98.70 & 98.52 \\
\hline Credit Approval & $\mathbf{8 8 . 0 5}$ & 86.68 & 86.68 \\
\hline Diabetic Retinopathy Debrecen & 79.06 & $\mathbf{7 9 . 4 1}$ & 78.62 \\
\hline Glass Identification & 84.11 & $\mathbf{8 4 . 5 6}$ & 83.18 \\
\hline Heart Disease Cleveland & $\mathbf{8 9 . 5 6}$ & $\mathbf{8 9 . 5 6}$ & $\mathbf{8 9 . 5 6}$ \\
\hline Hepatitis Domain & 92.86 & $\mathbf{9 6 . 4 3}$ & $\mathbf{9 6 . 4 3}$ \\
\hline Ionosphere & $\mathbf{9 8 . 0 1}$ & $\mathbf{9 8 . 0 1}$ & 97.73 \\
\hline BUPA Liver Disorders & 75.37 & $\mathbf{7 5 . 6 6}$ & 74.78 \\
\hline Ozon Level Detection & 98.86 & 99.13 & $\mathbf{9 9 . 1 9}$ \\
\hline Parkinsons Data Set & 94.36 & 94.87 & $\mathbf{9 6 . 4 1}$ \\
\hline Pima Indians Diabetes & $\mathbf{8 0 . 4 7}$ & 80.08 & 79.69 \\
\hline Thyroid Gland Data & $\mathbf{9 4 . 4 2}$ & $\mathbf{9 4 . 4 2}$ & $\mathbf{9 4 . 4 2}$ \\
\hline Vowel Recognition Data & 98.38 & 98.38 & $\mathbf{9 8 . 4 8}$ \\
\hline
\end{tabular}

Table 5.5: Differences from Best Accuracy.

\begin{tabular}{|l|c|c|c|}
\hline \multicolumn{1}{|c|}{ Dataset } & Algorithm 2 $(\infty)$ & Algorithm 2(1) & Algorithm 2E1 \\
\hline Breast Cancer Wisconsin Original & -0.29 & -0.29 & $\mathbf{0}$ \\
\hline Cardiotocography (type 1 vs.others) & -0.14 & -0.14 & $\mathbf{0}$ \\
\hline Cardiotocography (type 2 vs.others) & -0.14 & -0.23 & $\mathbf{0}$ \\
\hline Car Evaluation (type 1 vs.others) & -4.28 & -4.28 & $\mathbf{0}$ \\
\hline Car Evaluation (type 2 vs.others) & $\mathbf{0}$ & $\mathbf{0}$ & $\mathbf{0}$ \\
\hline Car Evaluation (type 3 vs.others) & -1.22 & -0.59 & -0.06 \\
\hline Car Evaluation (type 4 vs.others) & $\mathbf{0}$ & -0.06 & -0.18 \\
\hline Climate Model Simulation Crashes & $\mathbf{0}$ & -0.19 & -1.37 \\
\hline Credit Approval & $\mathbf{0}$ & -1.37 & -0.79 \\
\hline Diabetic Retinopathy Debrecen & -0.35 & $\mathbf{0}$ & -1.38 \\
\hline Glass Identification & -0.45 & $\mathbf{0}$ & $\mathbf{0}$ \\
\hline Heart Disease Cleveland & $\mathbf{0}$ & $\mathbf{0}$ & $\mathbf{0}$ \\
\hline Hepatitis Domain & -3.57 & $\mathbf{0}$ & -0.28 \\
\hline Ionosphere & $\mathbf{0}$ & $\mathbf{0}$ & -0.88 \\
\hline BUPA Liver Disorders & -0.29 & $\mathbf{0}$ & $\mathbf{0}$ \\
\hline Ozon Level Detection & -0.33 & -0.06 & $\mathbf{0}$ \\
\hline Parkinsons Data Set & -2.05 & -1.54 & $\mathbf{0}$ \\
\hline Pima Indians Diabetes & $\mathbf{0}$ & -0.39 & $\mathbf{0}$ \\
\hline Thyrod gland data & $\mathbf{0}$ & $\mathbf{0}$ & $\mathbf{0}$ \\
\hline Vowel Recognition Data & -0.1 & -0.1 & $\mathbf{- 1 . 3 8}$ \\
\hline \hline Largest Difference & -4.28 & -4.28 & $\mathbf{0 . 2 6 6 5}$ \\
\hline Average Difference & -0.6605 & -0.462 & $\mathbf{1 2}$ \\
\hline \# bests & 8 & 8 & \\
\hline
\end{tabular}

vs. the others. It reduces the average number of LPs by about $96.56 \%$ and $67.90 \%$ when compared to Algorithms 2( $\infty)$ and 2(1), respectively, and reduces the solution time by $94.77 \%$ when compared to Algorithm $2(\infty)$ and $71.31 \%$ when compared to 
Algorithm 2(1).

Algorithm 2E1 reduces solution time by an average 70-90\% compared to existing algorithms, while also slightly improving total accuracy on average.

Table 5.6: Algorithm speeds (number of LPs, seconds) for binary classification.

\begin{tabular}{|l|c|c|c|c|c|c|}
\hline \multirow{2}{*}{ Dataset } & \multicolumn{2}{|c|}{ Algorithm 2( $\infty)$} & \multicolumn{2}{c|}{ Algorithm 2(1) } & \multicolumn{2}{c|}{ Algorithm 2E1 } \\
\cline { 2 - 7 } & LPs & Sec & LPs & Sec & LPs & Sec \\
\hline Breast Cancer Wisconsin Original & 100 & 3.1 & 12 & 0.7 & $\mathbf{6}$ & $\mathbf{0 . 3}$ \\
\hline Cardiotocography (type 1 vs.others) & 35 & 4.3 & 4 & 0.6 & $\mathbf{2}$ & $\mathbf{0 . 3}$ \\
\hline Cardiotocography (type 2 vs.others) & 67 & 5.1 & 4 & 0.6 & $\mathbf{2}$ & $\mathbf{0 . 3}$ \\
\hline Car Evaluation (type 1 vs.others) & 2583 & 350.6 & 372 & 28.1 & $\mathbf{8}$ & $\mathbf{0 . 7}$ \\
\hline Car Evaluation (type 2 vs.others) & 462 & 28.7 & 69 & 4.5 & $\mathbf{1}$ & $\mathbf{0 . 1}$ \\
\hline Car Evaluation (type 3 vs.others) & 1458 & 183.2 & 203 & 13.8 & $\mathbf{1 0}$ & $\mathbf{0 . 9}$ \\
\hline Car Evaluation (type 4 vs.others) & 205 & 16.7 & 33 & 2.3 & $\mathbf{5}$ & $\mathbf{0 . 4}$ \\
\hline Climate Model Simulation Crashes & 77 & 3.2 & 7 & 0.3 & $\mathbf{5}$ & $\mathbf{0 . 2}$ \\
\hline Credit Approval & 1237 & 37.1 & 87 & 7.3 & $\mathbf{1}$ & $\mathbf{0 . 1}$ \\
\hline Diabetic Retinopathy Debrecen & 4723 & 625.2 & 238 & 25.6 & $\mathbf{1 6}$ & $\mathbf{1 . 4}$ \\
\hline Glass Identification & 292 & 3.1 & 33 & 1.5 & $\mathbf{7}$ & $\mathbf{0 . 3}$ \\
\hline Heart Disease Cleveland & 404 & 5.3 & 31 & 1.0 & $\mathbf{6}$ & $\mathbf{0 . 2}$ \\
\hline Hepatitis Domain & 16 & 0.4 & 4 & 0.2 & $\mathbf{2}$ & $\mathbf{0 . 1}$ \\
\hline Ionosphere & 104 & 4.2 & 7 & $\mathbf{0 . 3}$ & $\mathbf{5}$ & $\mathbf{0 . 3}$ \\
\hline BUPA Liver Disorders & 581 & 14.8 & 83 & 2.7 & $\mathbf{1 0}$ & $\mathbf{0 . 3}$ \\
\hline Ozon Level Detection & 542 & 159.7 & 14 & 4.1 & $\mathbf{7}$ & $\mathbf{2 . 1}$ \\
\hline Parkinsons Data Set & 103 & 2.7 & 7 & $\mathbf{0 . 4}$ & $\mathbf{4}$ & $\mathbf{0 . 4}$ \\
\hline Pima Indians Diabetes & 1325 & 41.7 & 153 & 6.7 & $\mathbf{1 2}$ & $\mathbf{0 . 5}$ \\
\hline Thyrod gland data & 61 & 1.3 & 12 & 0.4 & $\mathbf{6}$ & $\mathbf{0 . 2}$ \\
\hline Vowel Recognition Data & 146 & 4.0 & 14 & 0.5 & $\mathbf{7}$ & $\mathbf{0 . 3}$ \\
\hline
\end{tabular}

\section{Scenario II}

The results in this section show the superior performance of Algorithm 2E1 against four widely used binary classification models. The classification algorithms are applied to seven real-world medical and biological datasets for disease diagnosis. Table 5.8 reports algorithms' performance using different evaluation metrics with the best results bolded. The evaluation metrics column "Met." indicates the metrics such as Precision ("Pre."), Recall ("Re."), F1 score ("F1"), accuracy ("Acc."), and processing time in seconds ("Sec."). Note that for KNN-Tune, and SVM-Tune, "Sec." row reports the total of required time for hyperparameter tuning and the required processing time by the algorithms. To ensure uniformity, the same folds are used for all algorithms through the experiments.

For the selected biological datasets, which are recall-oriented tasks, Table 5.8 
Table 5.7: Percentage Reductions in LPs Solved and Solution Times by Using Algorithm 2E1 instead of original Algorithms 2( $\infty)$ and 2(1) for Classification.

\begin{tabular}{|l|c|c|c|c|}
\hline \multirow{2}{*}{ Dataset } & \multicolumn{2}{|c|}{$\begin{array}{c}\text { Algorithm 2E1 vs. } \\
\text { Algorithm 2( } \boldsymbol{\infty})\end{array}$} & \multicolumn{2}{c|}{$\begin{array}{c}\text { Algorithm 2E1 vs. } \\
\text { Algorithm 2(1) }\end{array}$} \\
\cline { 2 - 5 } & LPs & Sec & LPs & Sec \\
\hline Breast Cancer Wisconsin Original & 94.00 & 90.32 & 50.00 & 57.14 \\
\hline Cardiotocography (type 1 vs.others) & 94.29 & 93.02 & 50.00 & 50.00 \\
\hline Cardiotocography (type 2 vs.others) & 97.01 & 94.12 & 50.00 & 50.00 \\
\hline Car Evaluation (type 1 vs.others) & 99.69 & 99.80 & 97.85 & 97.51 \\
\hline Car Evaluation (type 2 vs.others) & 99.78 & 99.65 & 98.55 & 97.78 \\
\hline Car Evaluation (type 3 vs.others) & 99.31 & 99.51 & 95.07 & 93.48 \\
\hline Car Evaluation (type 4 vs.others) & 97.56 & 97.60 & 84.85 & 82.61 \\
\hline Climate Model Simulation Crashes & 93.51 & 93.75 & 28.57 & 33.33 \\
\hline Credit Approval & 99.92 & 99.73 & 98.85 & 98.63 \\
\hline Diabetic Retinopathy Debrecen & 99.66 & 99.78 & 93.28 & 94.53 \\
\hline Glass Identification & 97.60 & 90.32 & 78.79 & 80.00 \\
\hline Heart Disease Cleveland & 98.51 & 96.23 & 80.64 & 80.00 \\
\hline Hepatitis Domain & 87.50 & 75.00 & 50.00 & 50.00 \\
\hline Ionosphere & 95.19 & 97.62 & 28.57 & 66.67 \\
\hline BUPA Liver Disorders & 98.28 & 97.97 & 87.95 & 88.89 \\
\hline Ozon Level Detection & 98.71 & 98.68 & 50.00 & 48.78 \\
\hline Parkinsons Data Set & 96.12 & 96.30 & 42.86 & 74.36 \\
\hline Pima Indians Diabetes & 99.09 & 98.80 & 92.16 & 92.54 \\
\hline Thyrod gland data & 90.16 & 84.61 & 50.00 & 50.00 \\
\hline Vowel Recognition Data & 95.20 & 92.50 & 50.00 & 40.00 \\
\hline \hline Maximum Percent Reduction & 99.92 & 99.80 & 98.85 & 98.63 \\
\hline Average Percent Reduction & 96.56 & 94.77 & 67.90 & 71.31 \\
\hline
\end{tabular}

shows that Algorithm 2E1 provides the highest value for the Recall metric for all cases except the Hepatitis dataset. For the Hepatitis dataset, the proposed algorithm gives the second highest Recall value after SVM-Tune while requiring much less time than SVM-Tune. On average, the proposed method improves the Recall value by 9.87\%, 15.57\%, 20.12\%, 32.49\%, 18.16\%, and 5.66\% over LR, KNN-Def, KNN-Tune, NB, SVM-Def, and SVM-Tune. Moreover, the proposed method outperforms the other ML models by providing the highest accuracy for five datasets.

Table 5.9 indicates the difference from the best obtained results on each dataset. The last five rows report the number of best results obtained by each algorithm. As shown, outperforms the comparators by providing more successes. After SVM-Def, NB is the worst model, but is the fastest, requiring less time in five cases out of seven.

In Fig. 5.3, the ROC_AUC scores for the different methods are compared. For the BLD dataset, SVM-Tune and the proposed method provide the first and the second 
Table 5.8: Performance of binary classification models in terms of Precision, Recall, F1 score, accuracy, and processing time (10-fold cross validation).

\begin{tabular}{|c|c|c|c|c|c|c|c|c|}
\hline \multirow{2}{*}{ Dataset } & \multirow{2}{*}{ Met. } & \multirow{2}{*}{$\mathbf{L R}$} & \multicolumn{2}{|c|}{ KNN } & \multirow{2}{*}{ NB } & \multicolumn{2}{|c|}{ SVM } & \multirow{2}{*}{ 2E1 } \\
\hline & & & Def & Tune & & Def & Tune & \\
\hline \multirow{5}{*}{ BCW } & Pre. & 0.94 & 0.94 & 0.94 & 0.96 & 0.92 & 0.95 & 0.96 \\
\hline & Re. & 0.95 & 0.95 & 0.95 & 0.97 & 0.85 & 0.97 & 0.98 \\
\hline & F1 & 0.95 & 0.95 & 0.95 & 0.91 & 0.89 & 0.96 & 0.97 \\
\hline & Acc. & 0.95 & 0.95 & 0.95 & 0.92 & 0.9 & 0.96 & 0.97 \\
\hline & Sec. & 0.45 & 1.07 & 2.14 & 0.05 & 0.38 & 73.58 & 0.03 \\
\hline \multirow{5}{*}{ DRD } & Pre. & 0.81 & 0.73 & 0.84 & 0.82 & 0.81 & 0.82 & 0.83 \\
\hline & Re. & 0.66 & 0.59 & 0.5 & 0.28 & 0.54 & 0.63 & 0.67 \\
\hline & F1 & 0.73 & 0.65 & 0.63 & 0.41 & 0.65 & 0.71 & 0.73 \\
\hline & Acc. & 0.75 & 0.69 & 0.7 & 0.61 & 0.71 & 0.75 & 0.75 \\
\hline & Sec. & 0.47 & 0.14 & 1.75 & 0.05 & 2.1 & 909.21 & 1.26 \\
\hline \multirow{5}{*}{ HD } & Pre. & 0.73 & 0.68 & 0.71 & 0.75 & 0.73 & 0.73 & 0.92 \\
\hline & Re. & 0.75 & 0.78 & 0.68 & 0.79 & 0.63 & 0.67 & 0.87 \\
\hline & F1 & 0.74 & 0.73 & 0.7 & 0.77 & 0.68 & 0.7 & 0.89 \\
\hline & Acc. & 0.74 & 0.71 & 0.7 & 0.77 & 0.7 & 0.71 & 0.88 \\
\hline & Sec. & 0.41 & 0.08 & 0.78 & 0.05 & 0.26 & 1287.86 & 0.16 \\
\hline \multirow{5}{*}{ BLD } & Pre. & 0.67 & 0.73 & 0.7 & 0.59 & 0.72 & 0.73 & 0.69 \\
\hline & Re. & 0.6 & 0.65 & 0.62 & 0.26 & 0.75 & 0.75 & 0.79 \\
\hline & F1 & 0.64 & 0.69 & 0.66 & 0.36 & 0.73 & 0.74 & 0.74 \\
\hline & Acc. & 0.65 & 0.71 & 0.67 & 0.54 & 0.73 & 0.74 & 0.72 \\
\hline & Sec. & 0.33 & 0.08 & 0.16 & 0.05 & 0.13 & 722.07 & 0.22 \\
\hline \multirow{5}{*}{ Parkinson } & Pre. & 0.8 & 0.87 & 0.98 & 0.89 & 0.67 & 0.72 & 0.95 \\
\hline & Re. & 0.77 & 0.75 & 0.7 & 0.64 & 0.8 & 0.85 & 0.86 \\
\hline & F1 & 0.78 & 0.8 & 0.82 & 0.75 & 0.73 & 0.78 & 0.9 \\
\hline & Acc. & 0.79 & 0.82 & 0.84 & 0.78 & 0.7 & 0.76 & 0.91 \\
\hline & Sec. & 0.53 & 0.16 & 1 & 0.06 & 0.19 & 439.27 & 0.11 \\
\hline \multirow{5}{*}{ PID } & Pre. & 0.76 & 0.72 & 0.83 & 0.76 & 0.74 & 0.77 & 0.76 \\
\hline & Re. & 0.75 & 0.82 & 0.78 & 0.68 & 0.69 & 0.78 & 0.82 \\
\hline & F1 & 0.76 & 0.77 & 0.8 & 0.72 & 0.71 & 0.78 & 0.78 \\
\hline & Acc. & 0.76 & 0.75 & 0.81 & 0.73 & 0.72 & 0.78 & 0.78 \\
\hline & Sec. & 0.53 & 0.12 & 1.56 & 0.05 & 1.09 & 6559.19 & 0.84 \\
\hline \multirow{5}{*}{ Hepatitis } & Pre. & 0.82 & 0.93 & 1 & 0.91 & 0.57 & 0.73 & 0.97 \\
\hline & Re. & 0.79 & 0.47 & 0.59 & 0.75 & 0.64 & 0.83 & 0.8 \\
\hline & F1 & 0.81 & 0.62 & 0.74 & 0.82 & 0.59 & 0.77 & 0.87 \\
\hline & Acc. & 0.81 & 0.72 & 0.79 & 0.84 & 0.55 & 0.76 & 0.9 \\
\hline & Sec. & 0.36 & 0.07 & 0.67 & 0.06 & 0.14 & 1337.77 & 0.08 \\
\hline
\end{tabular}

highest ROC_AUC scores, respectively. As reported in Table 5.8, SVM-Tune is very slow. The processing times of the proposed method and SVM-Tune are 0.22 and 722.07 seconds to solve the classification problem for the BLD dataset. Algorithm 2E1 reduces the required time by $99.97 \%$ in this case. For the rest of the datasets, provides higher ROC_AUC scores than the comparators.

According to the experiments in Scenario I, new Algorithm 2E1 reduces the mean solution time by about $94.77 \%$ and $71.31 \%$ vs. Algorithms $2(\infty)$ and $2(1)$, respectively, while slightly improving accuracy on average. The better classification accu- 
Table 5.9: Differences from the best results. (10-fold cross validation).

\begin{tabular}{|c|c|c|c|c|c|c|c|c|}
\hline \multirow{2}{*}{ Dataset } & \multirow{2}{*}{ Met. } & \multirow{2}{*}{ LR } & \multicolumn{2}{|c|}{ KNN } & \multirow{2}{*}{ NB } & \multicolumn{2}{|c|}{ SVM } & \multirow{2}{*}{ 2E1 } \\
\hline & & & Def & Tune & & Def & Tune & \\
\hline \multirow{5}{*}{ BCW } & Pre. & -0.02 & -0.02 & -0.02 & 0 & -0.04 & -0.01 & 0 \\
\hline & Re. & -0.03 & -0.03 & -0.03 & -0.01 & -0.13 & -0.01 & 0 \\
\hline & F1 & -0.02 & -0.02 & -0.02 & -0.06 & -0.08 & -0.01 & $\mathbf{0}$ \\
\hline & Acc. & -0.02 & -0.02 & -0.02 & -0.05 & -0.07 & -0.01 & 0 \\
\hline & Sec. & 0.42 & 1.04 & 2.11 & 0.02 & 0.35 & 73.55 & 0 \\
\hline \multirow{5}{*}{ DRD } & Pre. & -0.03 & -0.11 & 0 & -0.02 & -0.03 & -0.02 & -0.01 \\
\hline & Re. & -0.01 & -0.08 & -0.17 & -0.39 & -0.13 & -0.04 & 0 \\
\hline & F1 & 0 & -0.08 & -0.1 & -0.32 & -0.08 & -0.02 & 0 \\
\hline & Acc. & 0 & -0.06 & -0.05 & -0.14 & -0.04 & 0 & 0 \\
\hline & Sec. & 0.42 & 0.09 & 1.7 & 0 & 2.05 & 909.16 & 1.21 \\
\hline \multirow{5}{*}{ HD } & Pre. & -0.19 & $\begin{array}{l}-0.24 \\
\end{array}$ & -0.21 & -0.17 & -0.19 & -0.19 & 0 \\
\hline & Re. & -0.12 & -0.09 & -0.19 & -0.08 & -0.24 & -0.2 & 0 \\
\hline & F1 & -0.15 & -0.16 & -0.19 & -0.12 & -0.21 & -0.19 & 0 \\
\hline & Acc. & -0.14 & -0.17 & -0.18 & -0.11 & -0.18 & -0.17 & 0 \\
\hline & Sec. & 0.36 & 0.03 & 0.73 & 0 & 0.21 & 1287.81 & 0.11 \\
\hline \multirow{5}{*}{ BLD } & Pre. & -0.06 & 0 & -0.03 & $\begin{array}{l}-0.14 \\
\end{array}$ & -0.01 & 0 & -0.04 \\
\hline & Re. & -0.19 & -0.14 & -0.17 & -0.53 & -0.04 & -0.04 & 0 \\
\hline & F1 & -0.1 & -0.05 & -0.08 & -0.38 & -0.01 & 0 & 0 \\
\hline & Acc. & -0.09 & -0.03 & -0.07 & -0.2 & -0.01 & 0 & -0.02 \\
\hline & Sec. & 0.28 & 0.03 & 0.11 & 0 & 0.08 & 722.02 & 0.17 \\
\hline \multirow{5}{*}{ Parkinson } & Pre. & -0.18 & -0.11 & 0 & -0.09 & -0.31 & -0.26 & -0.03 \\
\hline & Re. & -0.09 & -0.11 & -0.16 & -0.22 & -0.06 & -0.01 & 0 \\
\hline & F1 & -0.12 & -0.1 & -0.08 & -0.15 & -0.17 & -0.12 & 0 \\
\hline & Acc. & -0.12 & -0.09 & -0.07 & $\begin{array}{c}-0.13 \\
\end{array}$ & -0.21 & -0.15 & 0 \\
\hline & Sec. & 0.47 & 0.1 & 0.94 & 0 & 0.13 & 439.21 & 0.05 \\
\hline \multirow{5}{*}{ Pima } & Pre. & -0.07 & -0.11 & 0 & -0.07 & -0.09 & -0.06 & -0.07 \\
\hline & Re. & -0.07 & 0 & -0.04 & -0.14 & -0.13 & -0.04 & 0 \\
\hline & F1 & -0.04 & -0.03 & 0 & -0.08 & -0.09 & -0.02 & -0.02 \\
\hline & Acc. & -0.05 & -0.06 & 0 & -0.08 & -0.09 & -0.03 & -0.03 \\
\hline & Sec. & 0.48 & 0.07 & 1.51 & 0 & 1.04 & 6559.14 & 0.79 \\
\hline \multirow{5}{*}{ Hepatitis } & Pre. & -0.18 & -0.07 & 0 & -0.09 & -0.43 & -0.27 & -0.03 \\
\hline & Re. & -0.04 & -0.36 & -0.24 & -0.08 & -0.19 & 0 & -0.03 \\
\hline & F1 & -0.06 & -0.25 & -0.13 & -0.05 & -0.28 & -0.1 & 0 \\
\hline & Acc. & -0.09 & -0.18 & -0.11 & -0.06 & -0.35 & -0.14 & 0 \\
\hline & Sec. & 0.3 & 0.01 & 0.61 & 0 & 0.08 & 1337.71 & 0.02 \\
\hline \multirow{5}{*}{ \# bests } & Pre. & - & 1 & 4 & 1 & - & 1 & 2 \\
\hline & Re. & - & 1 & & - & - & 1 & 6 \\
\hline & F1 & 2 & - & 1 & - & - & 1 & 6 \\
\hline & Acc. & 2 & - & 1 & - & - & 2 & 5 \\
\hline & Sec. & - & - & - & 5 & - & - & 1 \\
\hline
\end{tabular}

racies reflect larger feasible subsets found by the algorithm when compared with its predecessors.

Scenario II shows that the new Algorithm 2E1 can be a great competitor for binary classification problems. There is always a trade-off between accuracy and speed of methods. Therefore, a model is selected based on the dataset and the task at hand. Algorithm 2E1 shows promising performance in Recall-oriented machine 
Figure 5.3: Scenario II ROC_AUC Scores.

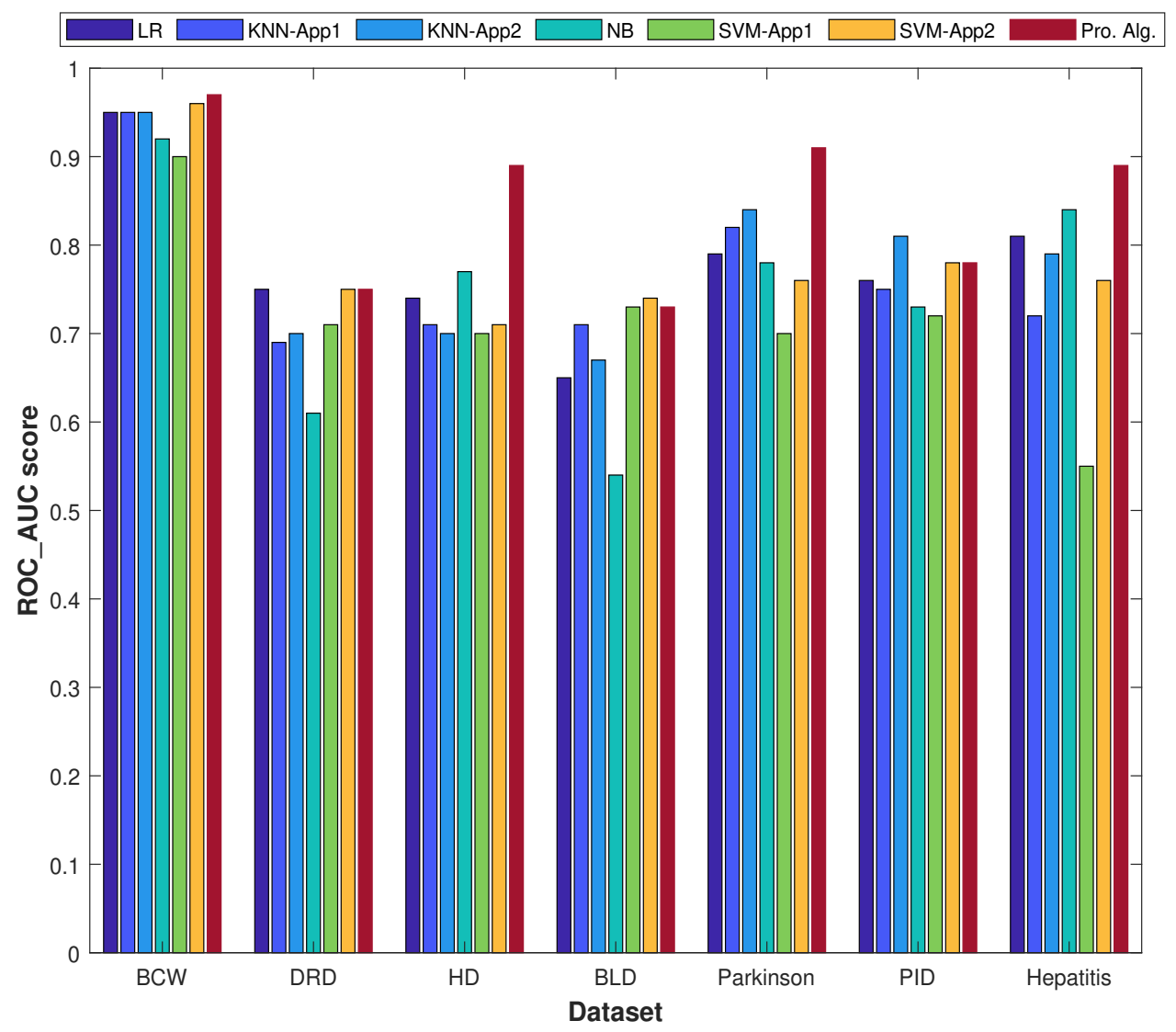

learning tasks such as cancer detection. 


\section{Chapter 6}

\section{Extended MAX FS for Sparse \\ Recovery in Compressive Sensing}

A sparse solution is one in which most of the variables have the value zero. The few variables that take nonzero values are called the support. Finding a sparse solution to an underdetermined system of linear equations is the central problem in Compressive Sensing (CS) signal recovery, and is cast as an instance of MAX FS [39] as follows: given the system $\mathbf{A x}=\mathbf{b}, \mathbf{x}=0$, find the maximum cardinality subset of $\mathbf{x}=0$ that permits a feasible solution to the original system. Several other formulations are also possible. Recognizing that MAX FS solution techniques can be used for sparse recovery, Jokar and Pfetsch [12] compared a number of MAX FS solution algorithms with state-of-the-art algorithms such as Basis Pursuit (BP) and Orthogonal Matching Pursuit (OMP) for sparse recovery of synthetic signals and concluded that Chinneck's algorithm based on solution for the MAX FS problem [11] provided the best results overall. Surprisingly, MAX FS solution methods have not been adopted for sparse recovery in CS. This motivates this research to evaluate MAX FS solution methods for use in the recovery phase of compressive sensing. 
In this chapter, Section 6.1 and 6.2 provide an introduction to CS and the existing CS sparse recovery algorithms, respectively. In Section 6.3, a faster version of Method M (refer to Section 2.1.4.4 for more details about Method M), which is called "Algorithm ME1E2( $\ell)$ " is developed by using the extensions introduced in Chapter 4. An example pipeline is developed for recovery of compressively sensed signals and is applied on real world signals to evaluate the performance of the existing MAX FS algorithms (Methods B and M) against the new Algorithm ME1E2( $\ell$ ). Because the focus is on compressed signal recovery, the signal acquisition part of the example pipeline is not optimized, and could be replaced with an improved version without any loss of generality. In all experiments, the new algorithms are compared with state-of-the-art CS sparse recovery methods.

The experiments consider two different applications: (i) recovery of compressively sensed ECG signals (Section 6.4), and (ii) recovery of compressively sensed speech signals (Section 6.5). The experiments are explained in Section 6.4.1 and Section 6.5.1. Ultimately, the superior performance of the existing and new MAX FS methods in addressing the main issues in sparse recovery such as: (1) small critical sparsity level and less compression for good recovery using the existing algorithms in CS, and (2) the quality of the recovered signal, are shown in Section 6.4.2 and Section 6.5.2.

\subsection{Compressive Sensing (CS)}

In order to ideally reconstruct a continuous time signal from its digital samples, the Shannon/Nyquist sampling theorem recommends a sampling rate higher than at least twice the bandwidth of the signal. Naturally occurring signals are often sparse with few components of the signal containing almost all the useful information. By choosing to sample just those components that contain the information, one can achieve 
dimensionality reduction. In standard dimensionality reduction techniques, all $n$ samples of signal $f$, obtained following the Shannon-Nyquist theorem are required; after applying a transform to the signal, the $S$ largest coefficients are identified, and the $(n-S)$ smallest coefficients are discarded; and the $S$ values and locations of the most significant coefficients are encoded. Unfortunately, this framework introduces some inherent inefficiencies. Firstly, in the case of high-bandwidth and high-resolution signals, the Nyquist rate is so high that the number of samples $n$ is large, even if $S$ is small. Secondly, all $n$ transform coefficients must be computed even though all except the $S$ largest coefficients will be discarded. Thirdly, the locations of the large coefficients must be encoded as well [71].

Compressive sensing (CS) addresses these inefficiencies by incorporating dimensionality reduction into the sensing process itself without going through the intermediate stage of acquiring all $n$ samples [72,73. This thesis does not address the acquisition of the samples from the analog domain using compressive sensing, instead it uses CS for reducing the number of samples used for archiving or for future signal processing. As a consequence, the input signals considered in this work are acquired respecting the Nyquist requirements.

In the signal processing context of this dissertation, the CS problem can be mathematically expressed as follows: Let $\mathbf{f} \in \mathbb{R}^{n}$ denote the original $n$ sample input signal which is $S$-sparse (i.e. has $S$ nonzeros and $S<<n$ ) or compressible with respect to a proper sparsifying basis, $\boldsymbol{\Psi}$. Therefore, $\mathbf{f}$ can have a sparse representation as follows:

$$
\mathbf{f}_{n \times 1}=\Psi_{n \times n} \mathbf{a}_{n \times 1}
$$

where $\Psi \in \mathbb{R}^{n \times n}$ and $\mathbf{a}$ is the sparse representation of the input $\mathbf{f}$. CS does not attempt to record all $n$ entries of the input signal. Instead, the signal $\mathbf{f}$ in a sparse 
domain is measured by sampling it according to a measurement matrix $\boldsymbol{\Phi} \in \mathbb{R}^{M \times N}$ which typically is designed with some element of randomness. Since these measurements are linear, the measurement vector $\mathbf{y}$ can be represented as

$$
\mathbf{y}_{m \times 1}=\boldsymbol{\Phi}_{m \times n}\left(\boldsymbol{\Psi}_{n \times n} \mathbf{a}_{n \times 1}\right) .
$$

In fact, Eqn. (6.2) explains the dimensionality reduction phase of CS when a long $S$-sparse vector, $\mathbf{a}_{n \times 1}$, is compressed to obtain $\mathbf{y}_{m \times 1}, S<m<<n$. Here, the goal is to obtain a number of measurements, $m$, as close to the sparsity level, $S$, as possible for greater compression.

In the signal recovery phase of CS, the measurement vector, $\mathbf{y}$, and the measurement matrix, $\boldsymbol{\Phi}$, are used to recover the input signal $\mathbf{f}$. As the number of measurements is much smaller than the length of the input signal, $m<<n$, this problem is underdetermined [74]. Despite the fact that $\boldsymbol{\Phi}$ is not full rank, when the input signal is $S$-sparse, $\mathbf{y}$ is just a combination of the $S$ nonzero components of a and their corresponding columns of the measurement matrix $\boldsymbol{\Phi}$. Therefore, by selecting the correct $S$ columns of $\Phi$ corresponding to the location of non-zeros in the sparse vector to form an appropriate full rank matrix, the sparse vector can be completely recovered.

However, there is no prior information about the location of nonzero components of the $S$-sparse signal, a, both during the compression phase or the recovery phase of CS. Therefore, a proper optimization technique to encode the location of the nonzero entries of the sparse signal serves a crucial role in estimating the sparse solution with high probability.

In the past few decades, a vast variety of sparse estimation methods have been proposed by researchers. Each of these methods has its own pros and cons. For 
instance, almost all of the existing optimization algorithms can recover the sparse solution when the input signal is very sparse or $m$ is very large relative to $S$. However, real-world signals are not often sparse enough or compressible with respect to a basis, $\boldsymbol{\Psi}$. Therefore, new optimization algorithms with higher critical sparsity are required. critical sparsity is the maximum number of nonzeros in the input signal beyond which the algorithm would fail in sparse recovery. The goal of this work is proposing sparse estimation techniques with higher critical sparsity, which allows recovery of more highly compressed signals, that is, larger $m$, while maintaining/improving the recovered signal quality when compared with the existing algorithms.

The two main phases of CS are explained below in more detail:

- Sparsification: Candes, Romberg, and Tao $[72]$ proved that sparsity of the input signal is one of the fundamental premises underlying CS that enables us to recover the desired signal with high probability.

Since real world signals in the time domain are not necessarily sparse, they must be sparsified using a suitable basis. e.g. Discrete Cosine Transform (DCT) or Discrete Wavelet Transform (DWT). The transformed signal coefficients, if sparse, should satisfy a power law decay. More precisely, if $\mathbf{f}_{n \times 1}=\boldsymbol{\Psi}_{n \times n} \mathbf{a}_{n \times 1}$ and the coefficients are sorted in descending order such that $\left|a_{1}\right| \geq\left|a_{2}\right| \geq \ldots \geq\left|a_{n}\right|$, then the signal is compressible if the magnitude of the sorted coefficients obeys the undermentioned power law

$$
\left|a_{i}\right| \leq \alpha i^{-q}
$$

where $\alpha>0$ is a constant and $q=1,2, \cdots$. To obtain an $S$-sparse signal, all but the $S$ largest coefficients are set to zero. 
- Signal Compression: After sparsifying the input signal $\mathbf{f}$ and obtaining the $S$-sparse signal a, linear projection of a with the length of $n$ can be obtained according to Eqn. (6.2). In practice, there is no priori information about the location of the $S$ nonzeros of a, so it is an NP-hard problem to choose $S$ columns of $\boldsymbol{\Phi}$ [71]. According to [75], if Egn. 6.4 is satisfied, then $\boldsymbol{\Phi}$ obeys the Restricted Isometry Property $(\mathrm{RIP}][76]$ and it is possible to recover the sparse input signal using an appropriate sparse signal recovery algorithm.

$$
\left(1-\delta_{S}\right) \leq \frac{\|\mathbf{\Phi} \mathbf{a}\|_{2}^{2}}{\|\mathbf{a}\|_{2}^{2}} \leq\left(1+\delta_{S}\right),
$$

where $\|\cdot\|_{2}$ denotes the $\ell_{2}$ norm and $\delta_{S} \in(0,1)$ is the restricted isometry constant. Although RIP is a sufficient condition for sparse recovery, checking RIP requires solving an NP-complete problem. Therefore, another simpler condition, which easily guarantees a stable solution, is mutual incoherence [74] and is given as follows 77 :

$$
\mu(\boldsymbol{\Phi}, \Psi)=\max _{1 \leq i \neq j \leq n}\left|<\widetilde{\Phi}_{i}, \Psi_{j}>\right|
$$

where $<., .>$ denotes the numerical operation of inner product between the column vectors $\phi_{i}, \psi_{j} \in \mathbb{R}^{n}$ of $\widetilde{\Phi}$ (or rows of $\boldsymbol{\Phi}$ ) and $\boldsymbol{\Psi}$. In this definition, both the matrices are considered to be orthonormal.

Based on [78], no information is lost during recovery if the length of the measurement vector $m$ is at least

$$
m \geq C \mu^{2}(\boldsymbol{\Phi}, \boldsymbol{\Psi}) S \log n
$$

where $C$ is a positive constant. Therefore, low coherence between the measure- 
ment matrix, $\boldsymbol{\Phi}$, and the sparsifying matrix, $\boldsymbol{\Psi}$, means that the input signal can be reconstructed from a shorter measurement vector. Mathematically speaking, $\mu^{2}(\boldsymbol{\Phi}, \boldsymbol{\Psi})$ equals one in a highly coherent situation and therefore as the two matrices become uncorrelated, the number of measurements needed to recover the signal will decrease.

The Compression Ratio $\overline{\mathrm{CR}}$ in percentage is defined as:

$$
C R=\left(1-\frac{m}{n}\right) \times 100
$$

Measurement matrices used in compressive sensing may be broadly divided into two groups: random measurement matrices and deterministic measurement matrices. Figure 6.1 shows the two main categories of measurement matrices: random measurement matrix and deterministic measurement matrix 79].

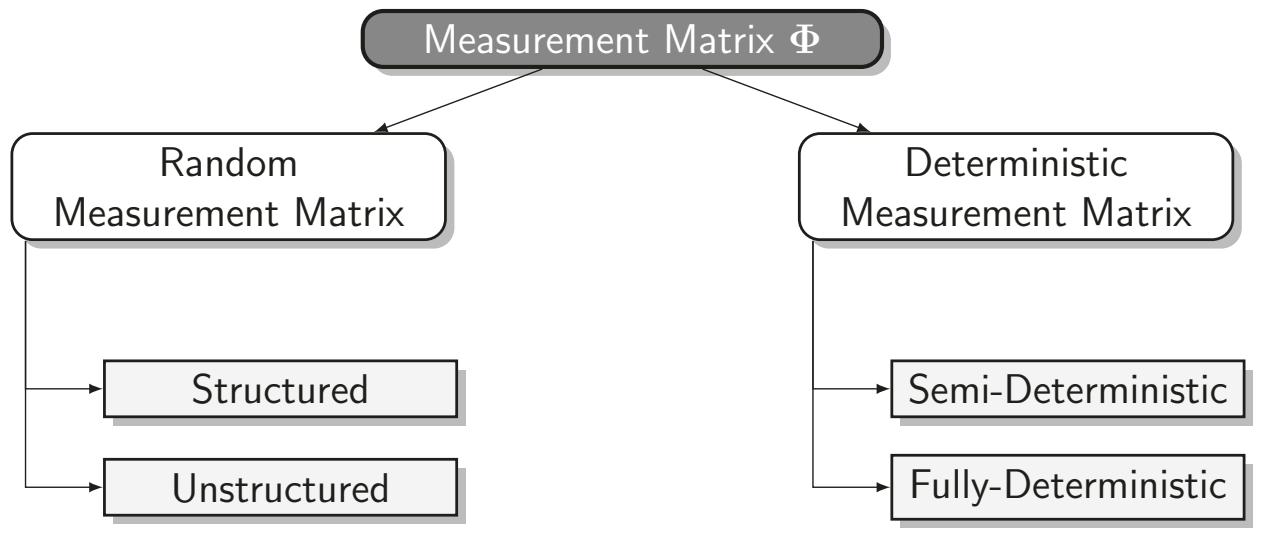

Figure 6.1: Different classes of measurement matrix $\Phi$.

Baraniuk et al. 80 showed that sparse recovery with high probability is guaranteed by using random matrices as they hold the RIP property. Random matrices with independent and identically distributed (i.i.d.) components drawn from Gaussian $\mathcal{N}\left(0, \frac{1}{m}\right)$ and sub-Gaussian Bernoulli distribution $\left( \pm \frac{1}{\sqrt{m}}\right)$ are two 
unstructured random sensing matrices which satisfy the RIP condition [81]. Unstructured random sensing matrices require more multiplies and adds to obtain the compressed measurement and also during recovery process. Further, using them also requires more storage which may affect the speed of recovery algorithms [82]. Measurement matrices with structure are desired for large scale problems because compressed measurements can be obtained with less computations. Consequently, a variety of designs of structured random matrices have been considered in 83 89].

Deterministic measurement matrices can be further divided into semideterministic and fully-deterministic categories. Different techniques have been proposed to design the deterministic matrices which maintain the RIP condition. Toeplitz-structured matrix $[90]$ and circular deterministic measurement matrices [91, 92 are examples of semi-deterministic matrices. Moreover, fullydeterministic matrices, along with construction techniques, can be found in the literature 93 98.

In some practical applications, deterministic measurement matrices are preferred to random measurement matrices as they allow fast implementation with reduced storage requirements. Random measurement matrices have the advantage that both compression and encoding can be achieved simultaneously which the deterministic matrices do not have. In fact, when privacy is an issue, a random measurement matrix is preferred.

- Sparse Signal Recovery: The goal of the signal recovery process is to recover the input signal a from the compressed signal $\mathbf{y}$ and $\boldsymbol{\Phi}$ with high quality. In a practical situation, information about the number of nonzero coordinates of $\mathbf{a}$, their locations, or their values are all completely unknown. A sparse recovery 
algorithm applied to $\mathbf{y}$ and $\boldsymbol{\Phi}$ can provide both the position and the location of the nonzero entries in the input signal if the input signal happens to be sufficiently sparse $[78]$. Knowing that the input signal a is sparse, it is hoped that recovering a sparse solution $\mathbf{x}$ from the measurements obtained from the underdetermined system of linear equations $\mathbf{y}=\mathbf{\Phi} \mathbf{x}$, leads to $\mathbf{x}$ that is identical to the input signal a or at least a close approximation to the input signal. For this reason, the output signal recovery process is also known as "sparse approximation" or "sparse recovery". Recovering a sparse solution from an underdetermined system of linear equations is NP-hard [99]. Sparse approximation seeks to minimize the cardinality of the support (number of nonzeros) in $\mathbf{x}$, commonly expressed as the zero "norm" which can be formulated as follows:

$$
\mathbf{x}=\underset{x}{\arg \min }\|\mathbf{x}\|_{0} \leqslant S \quad \text { s.t. } \quad \mathbf{y}=\mathbf{\Phi} \mathbf{x}
$$

where $\|.\|_{0}$ represents $\ell_{0}$ norm.

The sparse recovery problem has been the subject of extensive research over the last few decades in a great diversity of research communities, such as applied mathematics, statistics, and theoretical computer science, and for a variety of applications. Some applications of sparse recovery algorithms and CS theory include image compression 100 103, video coding 104,105], compressive radar 106, 107], compressive transmission data [108 111, machine learning [112 114, communication systems 115 118], and speech processing [119,120], just to name a few.

The focus of this work is on sparse recovery phase of CS. In the following sections, an overview of different categories of the existing sparse recovery methods, and the main steps of the state of the art algorithms are presented. 


\subsection{Existing Algorithms for CS Sparse Recovery}

Broadly speaking, sparse recovery algorithms can be categorized into three classes: convex optimization, non-convex techniques, and greedy algorithms 121. The state of the art algorithms of the mentioned classes is presented in Fig. 6.2 [122].

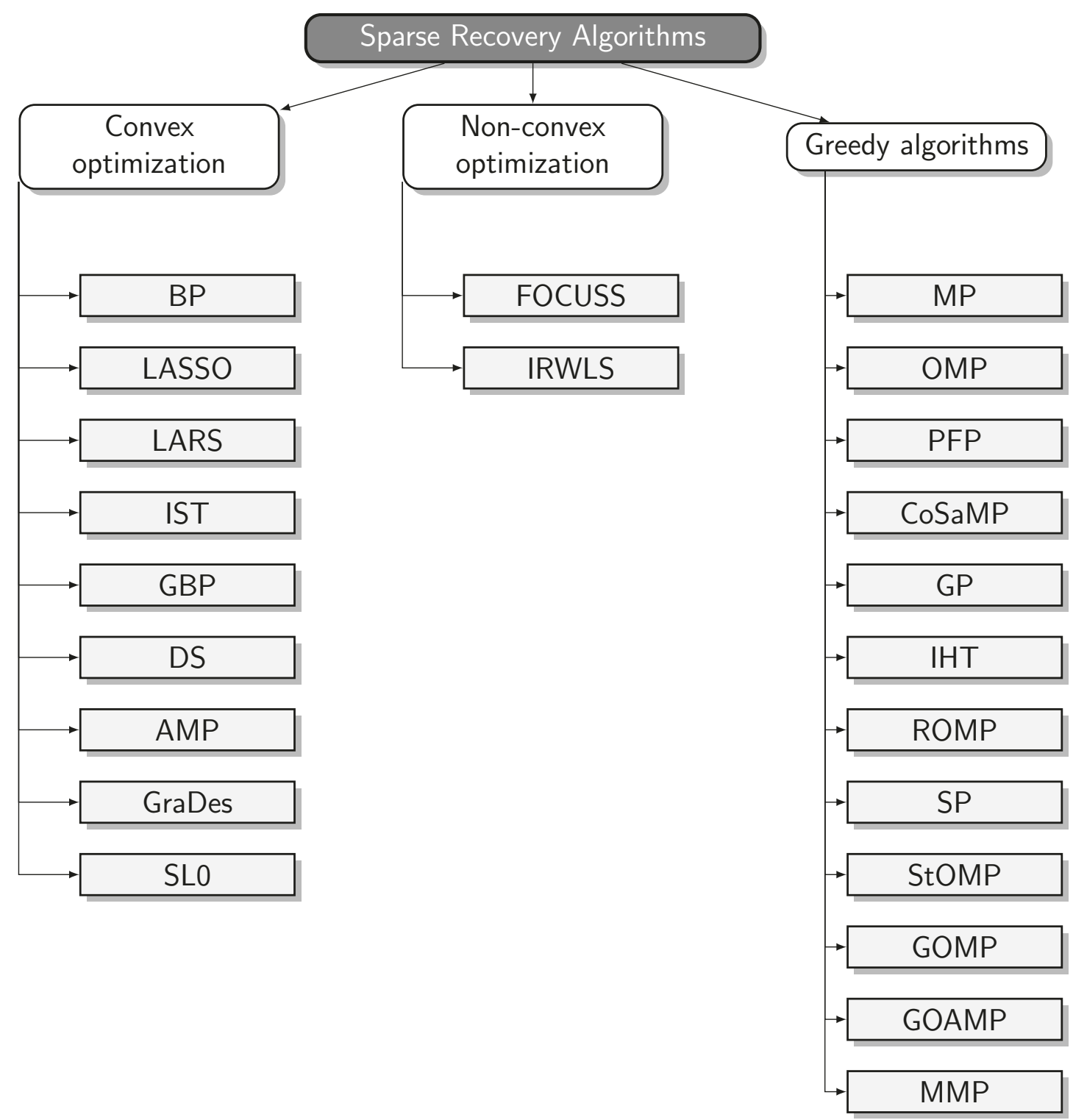

Figure 6.2: Classification of the existing sparse recovery algorithms.

In addition to the long list of mentioned algorithms, a wide variety of other sparse 
recovery algorithms exist in the literature. Some of them are: Bayesian Compressive Learning (BCS) [123], Normalized Iterative Hard Thresholding [124], Conjugate Gradient Iterative Hard Thresholding [125], Differential Orthogonal Matching Pursuit D-OMP [126, Back-tracking based Adaptive Orthogonal Matching Pursuit (BAOMP [127], Chaining Pursuit (CP [128, Fast Iterative Shrinkage Thresholding Algorithm (FISTA) [129], Forward-Backward Pursuit (FBP) [130], Hard Thresholding Pursuit [131], Heavy Hitters on Steroids (HHS) [132], $\ell_{p}$-Regularized Least-Squares Two Pass [133], Sequential Least Squares Matching Pursuit (SLSMP) 134], Sparse Adaptive Orthogonal Matching Pursuit (SpAdOMP [135), Sparse Reconstruction by Separable Approximation (SpaRSA [136], Stochastic Gradient Pursuit (SGP) [137], Stochastic Search Algorithms [138], Tree Search Matching Pursuit (TSMP) [139], and Vector Approximate Message Passing (VAMP) [140].

The rest of this subsection presents an overview of the different classes mentioned in Fig. 6.2.

\section{Convex Optimization}

The sparse recovery problem of CS can be cast as a convex optimization problem that may be solved using techniques such as linear programming (LP), projected gradient methods, interior-point methods, or iterative thresholding [141]. Convex optimization techniques are robust to noise, but are complex and challenging to apply as they have the worst case complexity around $O\left(m^{2} n^{3}\right)$ [43].

As minimizing the $\ell_{0}$ norm is NP-hard, most algorithms instead work to minimize some other norm $\|\mathbf{x}\|_{p}:=\left(\sum_{i=1}^{n}\left|x_{i}\right|^{p}\right)^{\frac{1}{p}}, \quad p \geq 0$. In the 1990's, using convex optimization based on the $\ell_{1}$ norm was proposed to recover $S$-sparse signals. Chen, Donoho, and Saunders 142 143 studied a convex optimization approach called Basis Pursuit (BP) that minimized the $\ell_{1}$ norm of $\mathbf{x}$. Basis Pursuit is extremely effective in returning $\mathbf{x}$ that matches the input $\mathbf{a}$, when $\mathbf{a}$ is very sparse. After this work, Tibshi- 
rani proposed Least Absolute Shrinkage and Selection Operator (LASSO 144] that estimated the $S$-sparse signal by minimizing the $\ell_{2}$ data error objective. LASSO is a quadratic method that can be applied in a variety of models and also in the presence of noise. In fact, LASSO and Basis Pursuit De-noising (BPDN) 145] are similar solutions developed by different research communities, namely the statistics community and signal processing community, respectively. Two convex relaxation approaches, namely the Least Angle Regression (LARS) [146] and Iterative Soft Thresholding (IST) 147] were introduced in 2004. In 2007, Greedy Basis Pursuit (GBP was proposed by Huggins et al. [148]. GBP like BP minimizes the $\ell_{1}$-norm and similar to greedy algorithms such as Matching Pursuit (MP) proceeds to find the sparse solution. A criterion based on computational geometry is used by GBP to select the next nonzero component in the representation. Also, GBP may remove the nonzero component that it has already been selected. Another convex sparse recovery algorithm called Dantzig Selector (DS) was proposed by Candes et al. [149] in 2007. Dantzig Selector is a $\ell_{1}$ regularization approach that estimates sparse signals by minimizing the maximum component of the gradient of the squared error function. For a large $S$, the prediction accuracy of DS seems to be similar to that of the LASSO method [150]. As the DS solves an LP and the Lasso solves a quadratic program, DS is recommended over the LASSO when $S$ is large. Based on the fact that convex optimization methods are expensive with respect to computations in large-scale problems, fast iterative thresholding algorithms were studied as alternatives, but they were not able to provide high quality sparse solutions like convex methods. Therefore, Donoho et al. [151] introduced a new iterative thresholding algorithm called Approximate Message Passing (AMP by applying some simple modifications to iterative thresholding methods. Gradient Descent with Sparsification GraDes is a convex optimization method that was proposed by Grag et al. [152] in 2009. This algorithm can find the sparse solution 
when LASSO or LARS fail in sparse recovery due to numerical problems. Moreover, Smoothed LO SLO [153] is another convex algorithm which is known as a fast sparse estimation algorithm that has at least the same accuracy as $\ell_{1}$ minimization methods.

Each of the above-mentioned algorithms has advantages and disadvantages. For instance, Arjoune et al. [44 compared different sparse recovery algorithms in the estimation of noisy, sparse signals with length of 1024 at different sparsity levels, $S$. They showed that the recovery error of the optimization algorithms such as BP and GraDes increases as sparsity increases, and in all cases, BP outperforms GraDes method in terms of recovery error. However, GraDes is faster than BP. Therefore, based on the application and the characteristics of the input signal, an appropriate technique for recovery should be selected. In general, BP and SL0 methods have been more frequently applied in the sparse recovery of CS signals than other mentioned convex optimization methods. Also, BP and SL0 provide better quality recovered signals 154 156. Therefore, these two algorithms are discussed in more detail here.

\section{Basis Pursuit (BP)}

Chen, Donoho, and Saunders 145 proposed a technique to recover a sparse vector by minimizing the $\ell_{1}-$ norm:

$$
\min \|\mathbf{x}\|_{1}=\sum_{i=1}^{n}|\mathbf{x}(i)| \quad \text { s.t. } \quad \mathbf{\Phi} \mathbf{a}=\mathbf{y} .
$$

This can be converted to a linear program (LP) by a change in variables $x_{i}=u_{i}-v_{i}$, where $u_{i}$ and $v_{i}$ are non-negative:

$$
\min \mathbb{1}^{T}(\mathbf{u}+\mathbf{v}) \quad \text { s.t. } \quad \boldsymbol{\Phi}(\mathbf{u}-\mathbf{v})=\mathbf{y}, \quad u_{i}, v_{i} \geq 0 .
$$

The resulting LP has $2 n$ variables and $m$ equations. Upon solving, each $x_{i}$ is 
obtained as $x_{i}=u_{i}-v_{i}$. BP has complexity $O\left(n^{3}\right)$ and it needs at least $O(S \log n)$ measurements to recover the signal with high quality 145, 157, 158].

\section{Smoothed $\ell_{0}$ Norm (SL0)}

The main idea of the Smoothed-L0 Norm (SL0) method 159] is to find the $\ell_{0}$ norm

of the given input $\mathbf{x}=\left[\begin{array}{lll}x_{1} & \ldots & x_{n}\end{array}\right]^{S}$ using a smooth function $F_{\sigma}(\mathbf{x})$. In order to understand the procedure, consider the following definition for $v(x)$ :

$$
v(x)=\left\{\begin{array}{ll}
1 & x \neq 0 \\
0 & x=0
\end{array} .\right.
$$

Using this definition, the $\ell_{0}$ norm can be written as $\|\mathbf{x}\|_{0}=\sum_{i=0}^{n} v\left(x_{i}\right)$. To avoid the discontinuity of the $\ell_{0}$ norm which is caused by $v$, define:

$$
f_{\sigma}(x)=\exp \left(\frac{-x^{2}}{2 \sigma^{2}}\right)
$$

Therefore,

$$
\lim _{\sigma \rightarrow 0} f_{\sigma}(x)=\left\{\begin{array}{ll}
1 & x \neq 0 \\
0 & x=0
\end{array} \rightarrow \lim _{\sigma \rightarrow 0} f_{\sigma}(x)=1-v(x) .\right.
$$

Now, $F_{\sigma}(\mathbf{x})$ can be defined as

$$
F_{\sigma}(\mathbf{x})=\sum_{i=1}^{n} f_{\sigma}\left(x_{i}\right)
$$

The $\sigma$ parameter controls the trade-off between approximation accuracy and smoothness. A larger $\sigma$ leads to a smoother $F_{\sigma}$ but a weak approximation. On the other hand, smaller $\sigma$ leads to a better approximation but less smooth $F_{\sigma}$. From the above 
equations, the following equation can be obtained:

$$
\lim _{\sigma \rightarrow 0} F_{\sigma}(\mathbf{x})=\sum_{i=1}^{n}(1-v(x))=n-\|\mathbf{x}\|_{0} .
$$

So, $\ell_{0}$ norm of the input $\mathbf{x}$ can be estimated as $\|x\|_{0} \approx n-F_{\sigma}(\mathbf{x})$. Based on Eqn. (6.14), minimizing the number of nonzeros in the solution is equivalent to maximizing the smooth function $F_{\sigma}$ for a proper small $\sigma$. Unfortunately for small values of $\sigma$, $F_{\sigma}$ is highly non-smooth with many local maxima. To escape from local maxima, SL0 uses Graduated Non-Convexity GNC producer, which starts when there are no local maxima, and the $\sigma$ is initially very large. Then, gradually $\sigma$ decreases to zero. The steepest ascent algorithm is applied for each value of $\sigma$ to maximize $F_{\sigma}$. The solution of the underdetermined system can be summarized as:

$$
\max F_{\sigma}(\mathbf{x}) \text { subject to } \Phi \mathbf{x}=y \text {. }
$$

\section{Non-convex Optimization}

It is empirically shown in 160 that by using $\ell_{p}$ norm minimization when $0<p<1$ fewer measurements are needed in comparison with the case that $p=1$. Also, nonconvex techniques have large critical sparsity. However, applying non-convex optimization can be challenging for high-dimensional problems due to their computational complexity [161]. Based on [43], the maximum complexity of non-convex optimization methods is same as that of convex optimization algorithms and is approximately $O\left(m^{2} n^{3}\right)$.

In 1997, the FOCal Underdetermined System Solution (FOCUSS) [162] was introduced to find the solution of the underdetermined system, $\mathbf{y}=\mathbf{\Phi} \mathbf{x}$. Then, Rao and 
Kreutz-Delgado 163 used FOCUSS algorithm for minimizing $\ell_{p<1}$. The approach was replacing the $\ell_{p}$ objective function by a weighted $\ell_{2}$ norm. In 2008, Chartrand and Yin proposed Iterative Re-weighted Least Squares (IRWLS) algorithm [164]. They showed that IRWLS needed only a smaller number of measurements and much less sparse input signals to have high quality recovery when compared to unregularized version of nonconvex algorithms such as FOCUSS. A summary of the IRWLS algorithm is provided as an example of an existing non-convex technique.

\section{Iterative Re-weighted Least Squares (IRWLS)}

A nonconvex variant of BP [160] has been shown to provide exact recovery with fewer measurements. The $\ell_{1}$ norm is replaced by the $\ell_{p}$ norm,

$$
\min _{\mathbf{x}}\|\mathbf{x}\|_{p}^{p} \quad \text { s.t. } \quad \mathbf{\Phi} \mathbf{x}=\mathbf{y}
$$

where $0<p<1$. Before Rao and Kreutz-Delgado [163] considered $p<1, p \geq 1$ was studied, replacing the $\ell_{p}$ cost function in Eqn 6.17 by a weighted $\ell_{2}$ norm:

$$
\min _{\mathbf{x}} \sum_{i=1}^{n} w_{i} x_{i}^{2} \quad \text { s.t. } \quad \mathbf{\Phi} \mathbf{x}=\mathbf{y}
$$

where the objective function is a first order estimate of the $\ell_{p}$ such that $w_{i}=$ $\left|u_{i}^{(n-1)}\right|^{p-2}$. Chartrand and Yin 164 proposed a particular regularization strategy that greatly improved the ability of the reweighted least-squares algorithm to recover sparse signals.

In [164], $\boldsymbol{\Phi}$ is assumed to have the unique representation property (any $m$ columns are linearly independent) $[162$. This property leads to a unique solution of $\mathbf{\Phi x}=\mathbf{y}$ having sparsity $\|\mathbf{x}\|_{0}=S$. The approach finds weights based on Eqn. 6.19 for each 
iteration $t$.

$$
w_{i}=\left(x_{i}^{2}+\epsilon_{t}\right)^{\frac{p}{2}-1}
$$

where $\epsilon_{t}$ is a sequence converging to zero, $\epsilon_{t} \in(0,1), 0 \leq p<2$ and $\mathbf{y}=\mathbf{\Phi x}$. Then, a unique solution of a convex optimization problem Eqn. 6.18 is obtained in which $\mathbf{x}_{t} \rightarrow \mathbf{a}$.

\section{Greedy Algorithms}

Greedy algorithms select a local optimum at each step, hoping to reach the global optimum solution in the end. Faster execution and lower computational complexity are some advantages of using this approach in sparse estimation. In general, the maximum complexity of the serial greedy algorithms and parallel versions of greedy techniques are $O(S m n)$ complexity and $O\left(S m n n_{i t r}\right)\left(n_{i t r}\right.$ stands for number of iterations), respectively [43]. Nonetheless, most of the greedy algorithms require prior knowledge of the sparsity level of the input signal, $S$, which is not available in many practical situations [165]. Also, greedy algorithms need more measurements than convex optimization algorithms. Fig. 6.3, which is a revised version of Figure 6 mentioned in 122, simply shows the main procedure of most greedy algorithms.

In 1993, a greedy algorithm called Matching Pursuit (MP) was proposed by Mallat and Zhang [166]. Then, a modification of MP, Orthogonal Matching Pursuit (OMP), was introduced by Pati et al. 167 to improve the convergence of MP. Both algorithms select only one column, or one atom, in each iteration. Therefore, these algorithms are classified under serial greedy algorithms. In 2006, Plumbley 168 proposed a greedy technique Polytope Faces Pursuit (PFP), that uses BP to approximate the sparse solution and is based on the geometry of the polar polytope. This method is in the style of MP, and its complexity is of a similar order to OMP. PFP addresses the complexity and compression issues of BP and the greedy algorithms such as MP 


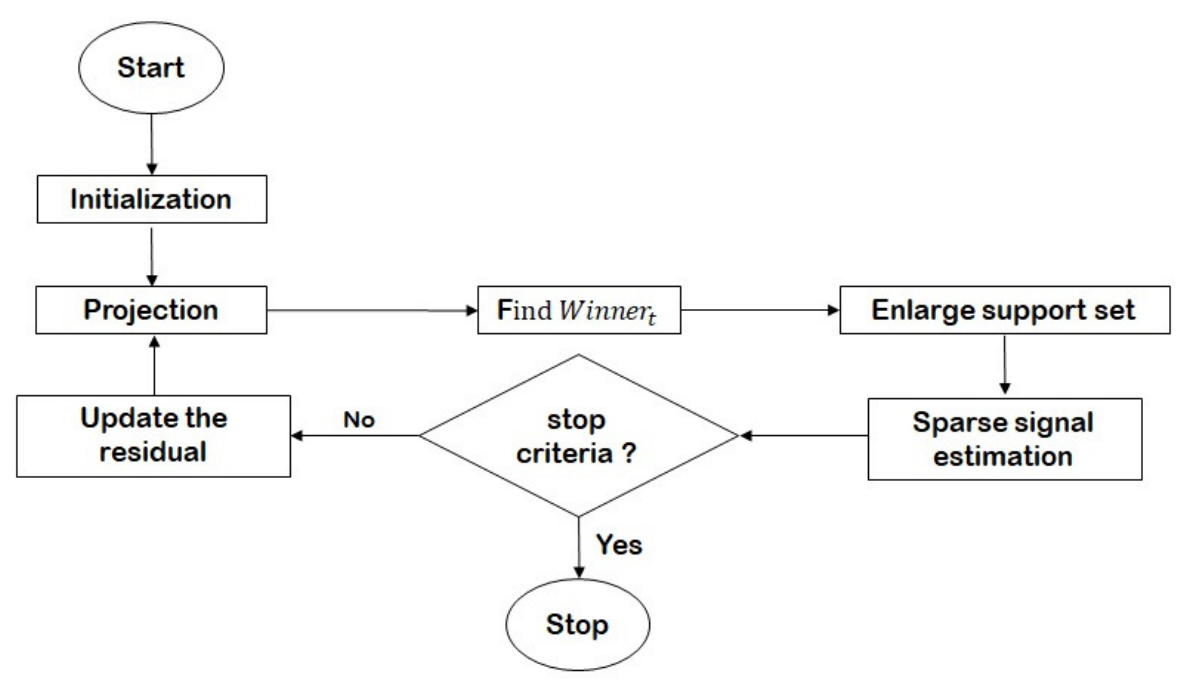

Figure 6.3: Procedure of greedy algorithms.

and OMP, respectively. It produces good results for the cases that are hard for MP. Another serial greedy algorithm called Gradient Pursuit (GP) was proposed as a modified version of OMP, in 2008, by Blumensath et al. [169]. Except for the update solution step, all the steps of the GP algorithm are as same as MP and OMP. GP outperforms MP and often shows a close performance to OMP.

GP is faster than OMP; therefore, applying GP for large scale problems is preferred over OMP. Another greedy algorithm is Compressive Sampling Matching Pursuit (CoSaMP [170 and it selects $k$ atoms instead of one in each iteration. CoSaMP is faster than OMP, and it guarantees the success of the sparse estimation in situations that OMP cannot [170]. A popular greedy-type method to estimate the sparse solution is Iterative Hard Thresholding (IHT) [171]. This method guarantees a lower approximation error compared with that of CoSaMP. Nevertheless, IHT requires an infinite number of iterations to reduce the error to zero even for noiseless observations. The difference between IHT and GP is in generating the support set. In GP, the selected atom cannot be re-evaluated and removed afterwards. Nevertheless, IHT 
can remove the added atoms in order to keep the dictionary elements with the largest magnitudes (the most significant elements) [169]. In 2009, Dai et al. proposed the Subspace Pursuit (SP) method [157] for sparse estimation in CS. They inferred that SP has a computational cost close to OMP, and an accuracy of the same order as LP methods. In each iteration, SP solves two least squares problem while OMP considers only one. Also, SP re-evaluates all the indexes at each iteration. Therefore, the previously selected atoms can be removed from the support set at any iteration, which is not the case in OMP [157]. Three OMP-based algorithms, namely Regularized Orthogonal Matching Pursuit (ROMP] [172], Stage-wise Orthogonal Matching Pursuit (StOMP [173], and Generalized Orthogonal Matching Pursuit (GOMP) 174] were proposed in order to improve the existing greedy algorithms. All the mentioned OMP-based methods guarantee a high quality sparse solution comparable to that of $\ell_{1}$ - minimization methods with the same or better speed when compared to OMP. On the other hand, Sun and Ni proposed an MP-based greedy method called Generalized Orthogonal Adaptive Matching Pursuit (GOAMP [175], in 2013. The significant feature of GOAMP is that the sparsity level of the input signal, $S$, which is unknown in most practical applications, is not required. In general, all the mentioned variations of OMP have indicated better empirical and theoretical performance compared to OMP. However, their performance in the presence of noise is far from being acceptable 176]. To solve the mentioned problem, Multipath Matching Pursuit MMP was proposed by Kwon et al [176], in 2014. MMP considers a greedy strategy to perform a tree search to find the sparse solution. In contrast to the different variations of OMP, MMP is an effective method in both noisy and noiseless scenarios. Also, the running time of the MMP is lower than that of other existing greedy algorithms [176].

Matching pursuit, orthogonal matching pursuit, and different variations of them have been widely used in the signal recovery phase in compressive sensing [44, 177. 
Knowing the steps and concepts of these algorithms can lead to a better understanding of a wide variety of other greedy algorithms, which are the direct extension of them. The overall main steps of some modified versions of the mentioned greedy algorithms, such as PFP and MMP, are presented here.

\section{Matching Pursuit (MP)}

Matching Pursuit [166] is an iterative greedy algorithm. In each iteration $t$, a column of $\boldsymbol{\Phi}, \phi_{\text {winner }}$, is selected that is best aligned with the residual vector, $\mathbf{r}_{t-1}$, where $\mathbf{r}_{\mathbf{0}}=\mathbf{y}$. The index of the best aligned column, winner, is identified according to Eqn. 6.20 [166].

$$
\text { winner }_{t}=\arg \max _{j=1, \ldots, n}\left|\frac{\phi_{j}^{T} \mathbf{r}_{t-1}}{\left\|\phi_{j}\right\|_{2}^{2}}\right|
$$

where (. $)^{T}$ is the transpose matrix and $\left\|\phi_{j}\right\|_{2}^{2}=1$ when columns of $\boldsymbol{\Phi}$ are normalized. The support, is enlarged by adding the index, winner of $_{t}$ the best aligned column, support $_{t}=$ support $_{t-1} \cup$ winner $_{t}$. In the next step, the support matrix $\boldsymbol{\Phi}_{\text {sup }}$ is updated as $\boldsymbol{\Phi}_{\text {sup }_{t}}=\left[\boldsymbol{\Phi}_{\text {sup }_{t-1}} \phi_{\text {winner }_{t}}\right]$. If winner $_{t} \in$ support $_{t-1}$, the support set and the support matrix are unchanged, i.e. support $t_{t}=$ support $_{t-1}$ and $\boldsymbol{\Phi}_{\text {sup }_{t}}=\boldsymbol{\Phi}_{\text {sup }_{t-1}}$.

After updating the support, the new residual vector and the sparse solution are calculated using Eqn. (6.21) and Eqn. 6.22).

$$
\begin{gathered}
x_{\text {winner } t}^{*}=\frac{\left(\phi_{\text {winner }_{t}}^{T} \mathbf{r}_{t-1}\right)}{\left\|\phi_{\text {winner }_{t}}\right\|_{2}^{2}}, \quad \mathbf{r}_{t}=\mathbf{r}_{t-1}-x_{\text {winner }_{t}}^{*} \phi_{\text {winner }_{t}} \\
x_{t_{\text {winner }_{t}}}=x_{t-1_{\text {winner }_{t}}}+x_{\text {winner }_{t}}^{*}
\end{gathered}
$$

The algorithm halts when the stopping condition is achieved (e.g. $\left\|\mathbf{r}_{t}\right\| \leq \epsilon$ ). MP has $O\left(m n n_{i t r}\right)$ complexity and the minimum number of measurements that is required by MP is $O(S \log (n / S))$ 178, 179.

\section{Orthogonal Matching Pursuit (OMP)}


Orthogonal Matching Pursuit (OMP) [167] is an improvement of MP. The main difference between MP and OMP is in estimating the sparse solution. In MP, the coefficients of the original sparse entries remains unchanged and the new coefficient that refers to the new member winner $_{t} \in a_{S}$ is selected as $x_{\text {winner }}^{*}$. In OMP, the sparse solution is obtained by solving a Least-Squares problem to re-evaluate all the coefficients in the sparse solution $\mathbf{x}$.

In each iteration of OMP, the residual vector $\mathbf{r}_{t}$ is orthogonal to the columns already selected. Therefore, no columns are selected twice. The inputs to this greedy algorithm are the measurement matrix $\boldsymbol{\Phi}$, the measurement vector $\mathbf{y}$, and the sparsity of the input signal $S[180]$. Note that the residual $\mathbf{r}_{t}$ is orthogonal to the column of $\boldsymbol{\Phi}_{\text {sup }_{t}}$. Therefore, a new element is selected at each step, and $\boldsymbol{\Phi}_{\text {sup }_{t}}$ has a full column rank. The OMP algorithm is summarized as in Fig. 6.4.

Figure 6.4 Orthogonal Matching Pursuit (OMP)

STEP 0: Initialization:

Iteration Counter: $t=1$.

Residual: $\mathbf{r}_{\mathbf{0}} \leftarrow \mathbf{y}$.

Index set: support $\leftarrow \emptyset$.

support matrix: $\boldsymbol{\Phi}_{\text {sup }} \leftarrow \emptyset$.

STEP 1: winner $_{t}=\arg \max _{j=1, \ldots, n}\left|\phi_{j}^{T} \mathbf{r}_{t-1}\right|$

STEP 2: Update the index set and the support matrix.

support $_{t}=$ support $_{t-1} \cup$ winner $_{t}$

$\boldsymbol{\Phi}_{\text {sup }_{t}}=\left[\boldsymbol{\Phi}_{\text {sup }_{t-1}} \phi_{\text {winner }_{t}}\right]$

STEP 3: Estimate the signal by solving a least-squares problem.

$\mathbf{x}_{t}=\arg \min _{\mathbf{x}}\left\|\boldsymbol{\Phi}_{\text {sup }_{t}} \mathbf{x}-\mathbf{y}\right\|_{2}$.

STEP 4: Update the measurement vector and the residual.

$\mathbf{y}_{t}=\boldsymbol{\Phi}_{\text {sup }_{t}} \mathbf{x}_{t}$

$\mathbf{r}_{t}=\mathbf{y}-\mathbf{y}_{t}$

STEP 5: If $t<S$, increment $t$ and go to Step 1 .

Output:

The support containing $T$ components from $\{1, \ldots, n\}$.

An estimated signal $\mathbf{x}$ containing nonzeros of the components in support. An approximated measurement vector, $\mathbf{y}_{T}$.

A residual $\mathbf{r}_{T}=\mathbf{y}-\mathbf{y}_{T}$. 
The goal is obtaining an output signal having a sparsity $T$ as close as possible to $S$. The minimum number of measurements that OMP needs for high quality sparse estimation is as same as for the BP algorithm, $O(S \log n)$, and it has $O(S m n)$ complexity $158,170,172,181]$.

\section{Polytope Faces Pursuit (PFP)}

The Polytope Faces Pursuit (PFP) algorithm 168 performs BP to find the sparse solution of the dual LP $\max _{\mathbf{c}}\left\{\mathbf{y}^{\mathbf{T}} \mathbf{c} \mid \mathbf{\Phi}^{\mathbf{T}} \mathbf{c} \leq \mathbf{1}\right\}$ and is in the style of the MP algorithm that adds one new basis vector at each step. PFP adopts a path following method through the relative interior of the faces of the polar polytope $P^{*}=\left\{\mathbf{c} \mid \Phi^{T} \mathbf{c} \leq\right.$ 1 ) associated with the dual LP problem and searches for the vertex $\mathbf{c}^{*} \in P^{*}$ that maximizes $\mathbf{y}^{T} \mathbf{c}$. The notation (.) $\dagger$ means pseudo-inverse matrix. The steps of the PFP algorithm are summarised in Fig. 6.5 [168].

Figure 6.5 Polytope Faces Pursuit (PFP)

STEP 0: Initialization:

Iteration counter: $t=1$.

Residual: $\mathbf{r}_{\mathbf{0}} \leftarrow \mathbf{y}$

Index set: support $\leftarrow \emptyset$.

Matrix of support: $\boldsymbol{\Phi}_{\text {sup }} \leftarrow \emptyset$.

$\mathrm{c}=0$.

STEP 1: Find face:

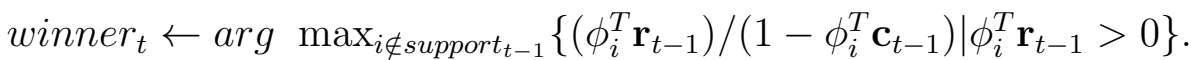

STEP 2: Add support:

$$
\begin{aligned}
& \text { support }_{t}=\text { support }_{t-1} \cup \text { winner }_{t} . \\
& \mathbf{\Phi}_{\text {sup }_{t}}=\left[\mathbf{\Phi}_{\text {sup }_{t-1}}, \phi_{\text {winner }_{t}}\right] \\
& \mathbf{X}_{t} \leftarrow\left(\boldsymbol{\Phi}_{\text {sup }_{t}}\right)^{\dagger} \mathbf{y} .
\end{aligned}
$$

STEP 3: If $\mathbf{x}_{t}<0$, remove the corresponding support.

Select $j \in$ support $_{t}$ such that $\mathbf{x}_{t_{j}}<0$; remove $\phi_{j}$ from $\boldsymbol{\Phi}_{\text {sup }_{t}}$.

Update support: support $_{t} \leftarrow$ support $_{t} \backslash\{j\}, \mathbf{x}_{t} \leftarrow\left(\boldsymbol{\Phi}_{\text {sup }_{t}}\right)^{\dagger} \mathbf{y}$.

STEP 4: $c_{t} \leftarrow\left(\boldsymbol{\Phi}_{\text {sup }_{t}}\right)^{\dagger T} \mathbf{1}, \mathbf{y}_{\mathbf{t}} \leftarrow \boldsymbol{\Phi}_{\text {sup }_{t}} \mathbf{x}_{t}, \mathbf{r}_{t} \leftarrow \mathbf{y}-\mathbf{y}_{t}$.

STEP 5: If termination condition is met (e.g. sparsity or residual) then exit. Else $t=t+1$ and go to Step 1 .

Output: $c=c_{t}, x=$ corresponding entries from $x_{t}$. 
This algorithm stops when the size of support reaches the maximum sparsity, $S$, or $\max _{i} \phi_{i}^{T} \mathbf{r}^{t-1}$ is smaller than the minimum residual condition, $\theta_{\min }$.

\section{Multipath Matching Pursuit (MMP)}

Multipath Matching Pursuit [176] is a greedy technique that executes the tree search. In MMP, a set of candidates, promising columns of matrix $\Phi$, should be tested. In contrast to MMP, only one candidate is considered heuristically in the OMP algorithm. Therefore, OMP is less computationally efficient than MMP. The steps of MMP are explained in Fig. 6.6 [176].

In the remainder of this section, the state of the art algorithms are discussed and compared with each other. A general comparison between three different classes of sparse recovery techniques is provided in Table 6.1. As mentioned in the table, convex and non-convex methods are not suitable for many problems as they have high computational complexity. On the other hand, greedy algorithms are faster, but their convergence to the sparse solution is not guaranteed. Also, prior knowledge of the input signal sparsity level is needed by greedy techniques. Non-convex optimization methods need a small number of measurements, which leads to more compression. In Table 6.2 122, the complexity and the minimum number of measurements of different algorithms are provided.

A large amount of research has been done to compare the performance of the state of the art sparse recovery algorithms in different applications. Some of the references containing CS recovery algorithms in different applications has been summarized in Table 6.3. In [182], the performance of SP, OMP, ROMP, GOMP, and GOAMP algorithms in image reconstruction have been compared. Experiments were performed for a compression ratio, $C R=50 \%$, with a random Gaussian matrix as the measurement matrix. The results were analysed based on Peak Signal to Noise Ratio (PSNR value. It was shown that GOAMP has better PSNR compared to the 
Table 6.1: Comparing three different categories of the sparse estimation algorithms.

\begin{tabular}{|c|c|c|}
\hline Approach & Complexity & Performance \\
\hline Convex optimization & $\approx O\left(M^{2} N^{3}\right)$ & $\begin{array}{l}\text { - Robust to noise } \\
\text { - Complex and slow } \\
\text { - Not suitable for large scale } \\
\text { problems }\end{array}$ \\
\hline Non-convex optimization & $\approx O\left(M^{2} N^{3}\right)$ & $\begin{array}{l}\text { - Requires fewer measurements } \\
\text { than } \ell_{1} \text { methods } \\
\text { - Complex and slow } \\
\text { - Not suitable for large scale } \\
\text { problems }\end{array}$ \\
\hline Greedy Algorithm & $\begin{array}{l}\text { Serial: } \quad O(M N k) \\
\text { Parallel: } O(M N . i t r)\end{array}$ & $\begin{array}{l}\text { - Low complexity and faster } \\
\text { - Requires prior knowledge of } \\
\text { signal sparsity } k \\
\text { - Convergence issues }\end{array}$ \\
\hline
\end{tabular}

Table 6.2: Complexity and minimum number of measurements of some state of the art sparse estimation algorithms.

\begin{tabular}{|c|c|c|c|}
\hline Algorithm & Complexity & $m$ & Reference \\
\hline $\mathrm{BP}$ & $O\left(n^{3}\right)$ & $O(S \log n)$ & \begin{tabular}{|l|l|l|}
145 & 157 & 158 \\
\end{tabular} \\
\hline MP & $O\left(m n n_{i t r}\right)$ & $O(S \log (n / S))$ & 166,179 \\
\hline$\overline{\mathrm{OMP}}$ & $O(S m n)$ & $O(S \log n)$ & $\overline{157}, \overline{158}, \overline{172} \overline{181}$ \\
\hline CoSaMP & $O(m n)$ & $O(S \log n)$ & $158,170,181$ \\
\hline IHT & $O(m n)$ & $O(S \log (n / S))$ & 125 \\
\hline ROMP & $O(S m n)$ & $O\left(S \log ^{2} n\right)$ & \begin{tabular}{|l|l|l|l|}
158 & 170 & 172 & 181 \\
\end{tabular} \\
\hline SP & $O(S m n)$ & $O(S \log (n / S))$ & $\overline{157}, \overline{158}$ \\
\hline StOMP & $O(n \log n)$ & $O(n \log n)$ & \begin{tabular}{|l|l|l|}
158 & 173 & 181 \\
\end{tabular} \\
\hline
\end{tabular}


Table 6.3: Comparative summary of CS recovery algorithms.

\begin{tabular}{|c|c|c|c|c|}
\hline Ref. & Algorithms & Application & $\Phi$ & Performance \\
\hline 182 & $\begin{array}{l}\text { SP } \\
\text { OMP } \\
\text { ROMP } \\
\text { GOMP } \\
\text { GOAMP }\end{array}$ & $\begin{array}{l}\text { Image } \\
\text { processing }\end{array}$ & $\begin{array}{l}\text { Random } \\
\text { Gaussian }\end{array}$ & $\begin{array}{l}\text { - GOAMP provides better Peak Sig- } \\
\text { nal to Noise Ratio (PSNR) com- } \\
\text { pared with others. }\end{array}$ \\
\hline 183 & $\begin{array}{l}\text { OMP } \\
\text { LARS }\end{array}$ & $\begin{array}{l}\text { Finding sparse solution } \\
\text { of random underdeter- } \\
\text { mined systems }\end{array}$ & $\begin{array}{l}\text { Random } \\
\text { Gaussian }\end{array}$ & $\begin{array}{l}\text { - OMP requires less time than } \\
\text { LARS to converge to the final so- } \\
\text { lution. } \\
\text { - When columns of the matrix } \Phi \\
\text { are highly correlated, LARS is pre- } \\
\text { ferred over OMP. }\end{array}$ \\
\hline 174 & $\begin{array}{l}\text { GOMP } \\
\text { OMP } \\
\text { StOMP } \\
\text { ROMP } \\
\text { CoSaMP }\end{array}$ & MIMO communication & $\begin{array}{l}\text { Random } \\
\text { Gaussian }\end{array}$ & $\begin{array}{l}\text { - GOMP has larger critical sparsity } \\
\text { compared with others. }\end{array}$ \\
\hline 176 & $\begin{array}{l}\text { OMP } \\
\text { StOMP } \\
\text { MMP } \\
\text { CoSaMP } \\
\text { BPDN }\end{array}$ & $\begin{array}{l}\text { Finding sparse solution } \\
\text { of random underdeter- } \\
\text { mined systems }\end{array}$ & $\begin{array}{l}\text { Random } \\
\text { Gaussian }\end{array}$ & $\begin{array}{l}\text { - MMP has performance close to } \\
\text { OMP for } \boldsymbol{\Phi}_{100 \times 256} \text { and up to the } \\
\text { sparsity level } S=20 \text {. } \\
\text { - MMP has better performance in } \\
\text { estimating } S \cong T \text { when the input } \\
\text { is less sparse }(S=30) \text {. Therefore, } \\
\text { it has higher critical sparsity than } \\
\text { others. } \\
\text { - MMP has the highest and the } \\
\text { OMP and the StOMP methods } \\
\text { have the lowest running time. }\end{array}$ \\
\hline 184 & $\begin{array}{l}\text { SL0 } \\
\text { FOCUSS } \\
\text { BP }\end{array}$ & $\begin{array}{l}\text { Sparse source sepa- } \\
\text { ration, blind source } \\
\text { separation (BSS) and } \\
\text { sparse component } \\
\text { analysis SCA }\end{array}$ & $\begin{array}{l}\text { Random } \\
\text { Normalized }\end{array}$ & $\begin{array}{l}\text { - SL0 provides same and sometimes } \\
\text { better accuracy. } \\
\text { - SL0 is faster. }\end{array}$ \\
\hline 120 & $\begin{array}{l}\text { CoSaMP } \\
\mathrm{BP}\end{array}$ & Speech processing & $\begin{array}{l}\text { Random } \\
\text { Normalized }\end{array}$ & $\begin{array}{l}\text { - BP provides better accuracy in } \\
\text { speech recovery. }\end{array}$ \\
\hline
\end{tabular}


Figure 6.6 Multipath Matching Pursuit (MMP)

STEP 0:

- Initialization:

Iteration counter: $t=1$.

Residual: $\mathbf{r}_{\mathbf{0}} \leftarrow \mathbf{y}$.

Index set: support $\leftarrow \emptyset$.

Candidate list: CandidateSet $\leftarrow \emptyset$.

- Input:

Measurement vector: $\mathbf{y}$

Measurement matrix: $\boldsymbol{\Phi}$

Input sparsity level: $S$

Size of expanding tree: $L$

Number of candidates to search: $k$

STEP 1: Set support $\leftarrow \emptyset$, winner $=0$ and $k=0$.

STEP 2: Choose $L$ best indices of columns by solving, $\left.\widetilde{\pi}:=\arg \max \| \Phi^{\mathbf{H}} \mathbf{b}_{t-1}^{k}\right) \|_{2}^{2}$. Then, set $j=1$.

STEP 3: Construct a temporary path:

$$
\text { support }^{\text {temp }}=\text { support }_{t-1}^{k} \cup \phi_{j}
$$

STEP 4: Check if the path already exists: if supportemp $\in$ support go to the next step.

otherwise, update:

winner $_{t}=$ winner $_{t}+1$, support $_{t}^{\text {winner }}=$ support $^{\text {temp }}$

support $_{t}=$ support $_{t} \cup$ support $_{t}^{\text {winner }}$

$\mathbf{x}_{t}^{\text {winner }_{t}}=\boldsymbol{\Phi}^{\dagger}\left(\right.$ support $\left._{t}^{\text {winner }}{ }_{t}\right) \mathbf{y}$

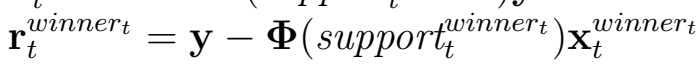

STEP 5: Set $j=j+1$. If $j \leq L$ then go to STEP 3 .

STEP 6: Set $k=k+1$. If $k \leq \mid$ support $_{t-1} \mid$ then go to STEP 2 .

STEP 7: Set $t=t+1$. If $t<S$ then go to STEP 1 .

STEP 8: Find the index of the best candidate:

$$
\begin{aligned}
& \text { winner }=\arg \min \left\|\mathbf{r}_{\text {winner }}^{S}\right\| \\
& \text { support }=\text { support }_{\text {winner }}^{\text {Sun }}
\end{aligned}
$$

Output: $x=\boldsymbol{\Phi}_{\text {support }}^{\dagger} \mathbf{Y}$

other techniques. In [183], a greedy algorithm, OMP, was compared with a convex technique, LARS, in estimating the sparse solution of the underdetermined system with a randomly generated measurement matrix using Gaussian distribution. Based on this work, the time taken to converge to the final solution by OMP is shorter 
than the LARS method. However, LARS outperforms OMP algorithm in the situation where some columns of $\Phi$ are highly correlated. A comparison between GOMP, OMP, StOMP, ROMP, and CoSaMP in the application of MIMO communication has been provided by Wang et al. 174. The experiments used a random Gaussian matrix as the measurement matrix and varied the input signal sparsity from $S=1$ to $S=70$. It has been shown that the critical sparsity of GOMP is larger than that of other mentioned sparse recovery algorithms. In [176], the quality of the sparse solution of OMP, StOMP, MMP, CoSaMP, and BPDN obtained for random, underdetermined systems have been compared. This work shows that MMP has larger critical sparsity and much longer convergence time. In [184], the superior performance of SL0 when compared with FOCUSS and BP (solved by interior-point LP solvers) was demonstrated in a a blind Source Separation (BSS) application. Moreover, [120] presented the results of CoSaMP and BP in speech enhancement and showed that BP outperforms CoSaMP in speech recovery quality.

As described in Section 2.2, existing MAX FS methods such as Method B provide better sparse solution and higher critical sparsity for an underdetermined system using synthetic datasets than BP and OMP [12]. However, MAX FS methods have not been used for any real-world applications due to their large processing time. For the first time, this research investigates the performance of the existing MAX FSbased methods in CS sparse recovery with respect to critical sparsity and recovered signal quality and also provides a comparative analysis against the performance of the well-known sparse recovery methods. The MAX FS methods are evaluated in the application of compressively sensed ECG and speech signals. Due to superior performance of the MAX FS methods in CS sparse recovery, techniques are introduced to improve the processing time of the existing MAX FS methods leading to a fast MAX FS algorithm. The experimental results show that the new faster MAX FS method 
significantly increases the speed of the existing MAX FS methods while preserving quality. The following sections detail the newly developed method and application of MAX FS methods in CS.

\subsection{New Extended MAX FS Algorithm for CS Re- covery}

As Chinneck's method for application of MAX FS for sparse estimation have shown much better performance [12], different new variations of the method such as Method $\mathrm{B}$ and Method M are studied here to show the value of applying MAX FS algorithms in CS. As mentioned in Chapter 4, the introduced extensions can be combined with any MAX FS algorithms to improve their speed. According to Section 4.2.2, the combination of E1 and E2 $(\ell)$ with the existing MAX FS method is faster than the other extensions. Therefore, the performance of Algorithm ME1E2 $(\ell)$ is investigated in this chapter for recovering compressively sensed real-world signals. Algorithm ME1E2( $\ell)$ has time complexity $O\left(n_{i t r} n^{3}\right)$ where $n_{i t r}$ indicates the number of iterations.

Algorithm ME1E2( $\ell$ ) assumes BP failure if the length of the first candidate list is greater than the defined threshold $\ell$. We set $\ell$ to $m-3$, as done for Method M (see Section 2.1.4.4). As shown in Fig. 4.3, Algorithm ME1E2( $\ell$ ) reduces the number of steps required by other MAX FS algorithms. In this algorithm, there is no inner/outer iteration to find the SupportSet. Therefore, the number of required LP solutions greatly decreases in this algorithm which significantly reduces the processing time. Note that the proposed method do not require prior knowledge of the input sparsity level.

In this chapter, the performance of the new and existing MAX FS algorithms are investigated and compared with that of conventional CS sparse recovery methods 
in recovery of compressively sensed ECG and speech signals. In Section 6.4.1 and Section 6.5.1, two pipelines are developed as examples to show how the proposed method can be applied for recovering compressively sensed signals.

\subsection{MAX FS for Sparse Recovery of Compres- sively Sensed ECG Signals}

The World Health Organization reports that Cardiovascular diseases (CVDs) cause approximately 17.9 million deaths globally each year [185]. In 2019, the American Heart Association $\mathrm{AHA}$ projected that the global death toll will increase to more than 23.6 million by 2030 [186]. Moreover, in the current pandemic situation due to COVID-19, patients with pre-existing cardiovascular disease, if infected by this novel Corona Virus, have higher risk of complications [187]. According to [188, there is a higher prevalence of CVD amongst COVID-19 patients. Consequently, AHA has supported Electrocardiogram (ECG) monitoring to optimize patient safety [189]. A wide variety of reliable wearable devices can be employed to measure ECG continuously. As continuous ECG measurement results in a large amount of data, bandwidth for transmission of data to the cloud for storage and storage requirements in the cloud will be demanding. Therefore, it is essential to compress the ECG measurements before transmission.

According to [190], compression algorithms for ECG signals can be classified into: (1) time-based algorithms such as Coordinated Reduction-Time-Encoding System (CORTES 191 and Amplitude Zone-Time-Epoch-Coding (AZTEC 192, and (2) transform-based algorithms, namely wavelet-based, and low-delay ECG compression algorithm (WLDECG) [190]. These compression algorithms can be power consuming due to complicated transforms [190]. A low power compression technique is compres- 
sive sensing, which senses and compresses data at the same time. The effectiveness of the CS approach to compress ECG signals has been investigated in the literature 156,193 .

For continuous monitoring of health parameters, it is advantageous from the power and transmission bandwidth perspectives to compress the signal at higher compression ratios. High compression rates result in fewer measurements and reduced storage and transmission requirements. To recover the highly compressed ECG signal with high quality, the critical sparsity of a CS sparse recovery algorithm must be large enough. In fact, it would be wiser to identify a recovery algorithm with higher critical sparsity prior to compressing the signal; hence, a signal can be compressed with a higher compression ratio. This motivated the investigation of MAX FS-based algorithms for recovery of compressively sensed ECG signals in order to provide higher critical sparsity compared to the existing CS sparse recovery methods.

Fig. 6.7 details a pipeline, which is as an example of how the proposed method can be used for CS sparse recovery. As described in the proposed pipeline, an input signal is segmented into frames with the length of $n$. Then, each segment is sparsified by applying a sparsifying basis, the Discrete Cosine Transform (DCT) as suggested in 156$]$. The sparsified segment is compressed using a random measurement matrix $\boldsymbol{\Phi}$ to compress each segment to a measurement vector $\mathbf{y}$. To recover the compressively sensed signal, the newly developed Algorithm ME1E2( $\ell$ ) (or other sparse recovery method) is applied to find the sparse solution, $\mathbf{x}$, for the underdetermined system, $\mathbf{y}=\mathbf{\Phi} \mathbf{x}$. Note that $\boldsymbol{\Phi}$ and $\mathbf{y}$ are known in the recovery phase.

The main contributions of this section are:

- Investigating the state-of-the-art MAX FS algorithms (Method B and Method $\mathrm{M})$ in recovery of the compressively sensed ECG signals for the first time. 
Figure 6.7 Pipeline for compressing and recovering a compressively sensed ECG signal.

\section{Signal Acquisition:}

Input: Input signal f.

0: Segment input signal, $\mathbf{f}$, into segments of length $n$.

for each segment do:

Sparsify each segment using DCT to find $\mathbf{a}_{S}$ ( $\mathbf{a}_{S}$ is a vector with $S$ nonzeros).

Compress each sparsified segment using a random measurement matrix $\boldsymbol{\Phi}$ to obtain measurement vector $\mathbf{y}$.

\section{end for}

Signal Recovery:

Input: $\boldsymbol{\Phi}_{m \times n}$ and $\mathbf{y}_{m \times 1}$.

1: Select a CS recovery algorithm (e.g. Algorithm ME1E2( $\ell$ ) in Fig. 4.3).

for each segment do:

Apply a sparse recovery algorithm.

Take the inverse DCT of the estimated sparse segment.

end for

2: Concatenate segments to obtain recovered signal $\hat{\mathbf{f}}$.

Output: The recovered signal $\hat{\mathbf{f}}$.

- Comparing the critical sparsity of the MAX FS algorithms and state-of-the-art CS recovery methods using ECG segments.

- Applying the proposed pipeline mentioned in Fig. 6.7 on real-world ECG signals.

- Evaluating the performance (speed, recovered signal quality) of the newly developed Algorithm ME1E2 $(\ell)$ and comparing that vs. the existing MAX FS algorithms in recovery of the compressively sensed ECG signals.

The following Section 6.4.1 and Section 6.4.2 explain the experimental setup and the empirical results, respectively. 


\subsubsection{Experimental Setup}

This section explains the experimental set up for recovery of compressively sensed ECG signals.

\subsubsection{Sparsifying and Measurement}

DCT is used to sparsify the input signal. Then, the sparse signal is measured using random measurement matrices, Random Normalized Matrices (RNM). The RNM is constructed from a matrix with elements that are chosen from a uniform random distribution between -1 and 1 ; each column is then normalized. Similar random matrices, which satisfy the RIP condition [81], have been applied in many articles; see, e.g., 32,76.

\subsubsection{ECG Database Description}

A total of 47 ECG signals that contain 22 women and 25 men at different age groups are taken from the MIT-BIH Arrhythmia Database [194]. A total of 18 selected ECG records $(104,107,111,112,115,116,118,119,201,207,208,209,212,213,214$, 228, 231, and 232) are pathologically significant [195]. Digitization of the records was done at a rate of 360 samples/second per channel over a range of $10 \mathrm{mV}$ with 11-bit resolution $194,196$. . For the analysis presented in this study, the first 3600 samples from each ECG signal are considered. Then, ECG signals are divided into segments of length $n=360$, each of which contains at least one ECG cycle. Consequently, 47 ECG signals each comprised of 10 segments of length $n=360$ are used in the experiments. 


\subsubsection{Performance Evaluation Metrics for ECG Recovery}

In addition to $S_{e}$ (refer to Eqn. (4.1)), the following metric is used to evaluate the quality of the signals returned by sparse recovery algorithms in recovery of compressively sensed ECG signals.

- Percentage Root Mean Square Difference (PRD). PRD is a popular metric for evaluating ECG signal quality [197]. It evaluates the difference between the original and the recovered ECG signal [198]. Percentage Root Mean Square Difference is calculated as:

$$
P R D=\frac{\|\hat{\mathbf{f}}-\mathbf{f}\|_{2}}{\|\mathbf{f}\|_{2}} \times 100 \%
$$

\subsubsection{Comparators}

In addition to the new extended Algorithm ME1E2( $\ell)$, the state-of-the-art MAX FS methods B, C and M are investigated for recovery of compressively sensed ECG signals for the first time. Moreover, the performances of the mentioned MAX FS algorithms are compared with representative algorithms from three different categories of CS sparse recovery algorithms: BP, MP, OMP, PFP, and IRWLS. More recent algorithms such as IHT and Look Ahead Orthogonal Matching Pursuit (LAOMP [199] are excluded since they require prior knowledge of the input sparsity, $S$, which is not available in practice. FBP is also excluded because it has much worse performance in our experiments in recovering compressively sensed speech signals in comparison to the chosen conventional comparators. 


\subsubsection{Software}

All algorithms are implemented in Matlab version 2018, running in a Windows 10 environment. The linear programming solver is MOSEK via the MOSEK Optimization Toolbox for Matlab version 8.1.0.56 [47]. Comparator algorithms were implemented using SparseLab [200], except for IRLWS which uses the code available in 201,202].

\subsubsection{Hardware}

The computations are carried out on a $3.40 \mathrm{GHz}$ Intel core $i 7$ machine with $16.0 \mathrm{~GB}$ RAM, running Windows 10.

\subsubsection{Results and Discussion}

This section focuses on recovery of compressively sensed ECG signals. In Section 6.4.2.1, the critical sparsities of the different optimization techniques are investigated. Then, the quality of the recovered whole ECG signal is evaluated in Section 6.4.2.2. Moreover, the performances of the new Algorithm ME1E2 $(\ell)$ and existing MAX FS methods are compared with that of the existing state-of-the-art algorithms through the results and discussion section.

\subsubsection{Critical Sparsity and Compression Degree of MAX FS Methods in Compressively Sensed ECG Recovery}

The goal of CS sparse recovery is to estimate a solution with sparsity level $T$ as close as possible to the input sparsity level, $S$. The critical sparsity levels of the existing sparse recovery algorithms are not large enough to handle an input which is insufficiently sparse. Therefore, sparse recovery algorithms increasingly fail to find $T \cong S$ as the input sparsity level increases. Consequently, a larger number of measurements and 
hence less compression, are required to prevent failure of recovery algorithms.

Jokar and Pfetsch [12] considered three random instances to build a system $\mathbf{b}=\mathbf{A x}$. Random vectors, $\mathbf{x}$, with different cardinalities, $S=\{10,15, \ldots, 80\}$, were generated for their experiments. The support entries were selected at random and the components of $\mathbf{x}$ were drawn from a normal distribution with zero mean and variance equal to one. Then, several methods were experimentally evaluated for estimating sparse solutions of the underdetermined system, $\mathbf{A x}=\mathbf{b}$, for different cases of $\mathbf{A}_{m \times n}$ such as deterministic, Hadamard, and random. In general, their experimental results for $\mathbf{A}_{128 \times 256}$, where the input signal was $S$-sparse can be summarized as follows: (1) BP had the worst critical sparsity and failed after $S=40$, (2) a variation of Chinneck's method, method B, was the best heuristic with the largest critical sparsity of $S=65$.

In this section, the critical sparsity levels of different optimization techniques are found for real-world ECG signals. The results are averaged over 100 ECG segments of length of $n=360$. Segments are chosen from the selected dataset consisting of 47 ECG signals of length 3600. All 47 ECG signals are segmented into 10 frames, each of length 360. Then, 2 segments are randomly selected from each of the 47 ECG signals, which results in a total of 94 ECG segments. The remaining 6 segments are randomly chosen from 6 subjects from the dataset, which consist of pathological cases $(104,107,111$, $112,115,116)$. Each segment has been manually checked to have at least one complete heart beat. In Fig. 6.8, a sample segment of a "pathologically significant" signal from the MIT-BIH Arrhythmia Database called " $104 \mathrm{~m}$ " is presented. As shown, the segment contains one ECG cycle.

Each ECG segment is sparsified using DCT at 19 different input sparsities $S=$ $\{10,15,20,25,30, \ldots, 100\}$ (e.g. $S=30$ means that only 30 out of 360 components of the ECG segment are nonzeros). It is observed that if the output signal sparsity, $T$, 

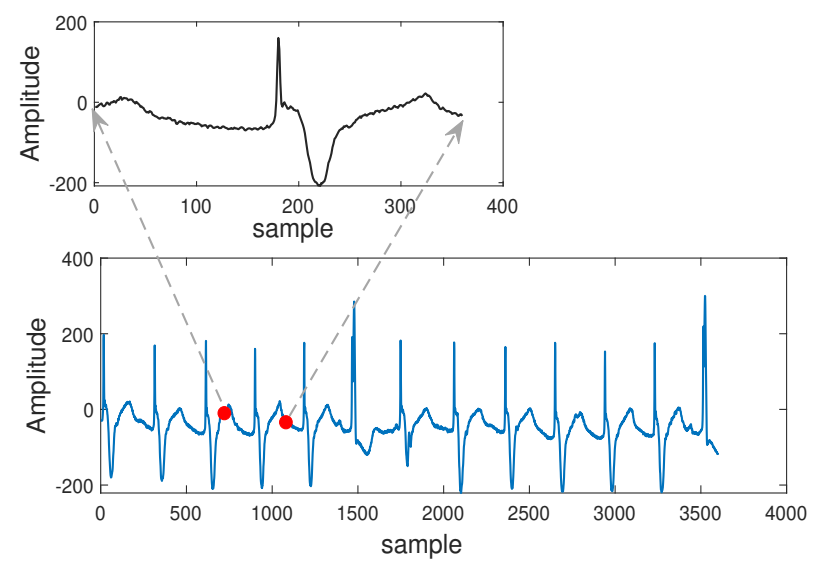

Figure 6.8: The waveform of ECG signal 104m and one segment of length 360.

equals the input signal sparsity, $S$, then the signals are also identical, so the matching of the signal sizes is used as the measure of success. Failure is declared when $T>S$.

The first column of Table 6.4 and Table 6.5 shows the sparsity level $S$ and the percentage of input sparsity $(s p \%)$ in parentheses. For instance, the sparsity level of the first and the last row of the table are $\frac{10}{360} \times 100=2.78 \%$ and $\frac{100}{360} \times 100=27.78 \%$, respectively. The remaining columns of Table 6.4 and Table 6.5 present the average sparsity error in percent $S_{e}$ of the output signal sparsity vs. the given input sparsity over 100 segments. $S_{e}=0 \%$ if the algorithm recovers a signal with sparsity the same as the original sparsity, $T=S$, in all 100 trials. The number of successes is shown in parentheses and complete success is shown in boldface. For instance, the average estimated sparsity level using the three MAX FS algorithms for $S=30$ is equal to 30 in Table 6.4. Consequently, the average sparsity error is equal to $0 \%$ and all algorithms succeed in all cases. For $S=75$, the average estimated sparsity levels over 100 ECG segments using Method B, Method M, and Algorithm ME1E2( $\ell$ ) are 77.11, 76.98, and 75.05, respectively. As a result, $S_{e}$ using Method B, Method M, and Algorithm ME1E2( $\ell)$ is $2.81 \%, 2.64 \%$ and $0.07 \%$, respectively. 
The last three rows in Table 6.4 and Table 6.5 have the following meanings: "\# Success" expresses the total number of successes over each column, "Critical Sparsity" shows the maximum sparsity level that an algorithm succeeds in all 100 cases, and "Min $m$ " indicates the minimum number of measurements needed for each algorithm to succeed in estimating sparsity equal to $S$.

In Table 6.4, the critical sparsity of Algorithm ME1E2( $\ell), 70$ (19.44\%), is higher than that of Method B and Method M, which have critical sparsities of $65(18.06 \%)$. Moreover, Algorithm ME1E2( $\ell$ ) provides a larger number of successes and it requires fewer measurements compared to Method B and Method M. Table 6.5 illustrates the performance of the well-known CS sparse recovery methods. IRWLS with the critical sparsity of $10(2.78 \%)$ has a poor performance in sparse recovery and is excluded from the tables. Based on Table 6.5, BP and MP have the highest and smallest critical sparsities of $55(15.28 \%)$ and $15(4.17 \%)$, respectively.

Table 6.6 reports the speed of the MAX FS algorithms in terms of number of LPs, "LPs", and processing time of the methods in seconds, "Sec". The best results are bolded. Based on the experimental results, Algorithm ME1E2 $(\ell)$ needs fewer LPs and accordingly less time to estimate sparse solution compared to Method B and Method M. Moreover, Table 6.7 analyses the speed of the algorithms by indicating the percentage reduction in number of LPs and processing time of the proposed Algorithm ME1E2( $\ell$ ) against Method B and Method M for each given sparsity level. To illustrate, Table 6.6 shows that the processing time of Method B and Method M for $S=60$ is about 14.44 seconds. Therefore, using Algorithm ME1E2( $\ell$ ) leads to a reduction of $98.27 \%$ in the required processing time when compared to Method B and Method M, as indicated in Table 6.7. The last two rows of Table 6.7 present the average improvement "Average Imp." and the maximum improvement "Max Imp." by Algorithm ME1E2( $\ell$ ) vs. the comparators. Algorithm ME1E2 $(\ell)$ reduces 
Table 6.4: Comparision of the performances of Method B, Method M, and Algorithm ME1E2 $(\ell)$ using the average sparsity error (\%) in estimating the sparsity level of 100 ECG segments of length $n=360$ at $C R=50 \%$.

\begin{tabular}{|c|c|c|c|}
\hline \multirow{2}{*}{$\mathrm{S}(\mathrm{sp} \%)$} & \multicolumn{3}{|c|}{$S_{e}(\%)$} \\
\hline & B & $\mathbf{M}$ & ME1E2( $\ell)$ \\
\hline $10(2.78 \%)$ & $\mathbf{0}^{(100)}$ & $\mathbf{0}^{(100)}$ & $\mathbf{0}^{(100)}$ \\
\hline $15(4.17 \%)$ & $\mathbf{0}^{(100)}$ & $\mathbf{0}^{(100)}$ & $\mathbf{0}^{(100)}$ \\
\hline $20(5.56 \%)$ & $\mathbf{0}^{(100)}$ & $\mathbf{0}^{(100)}$ & $\mathbf{0}^{(100)}$ \\
\hline $25(6.94 \%)$ & $\mathbf{0}^{(100)}$ & $\mathbf{0}^{(100)}$ & $\mathbf{0}^{(100)}$ \\
\hline $30(8.33 \%)$ & $\mathbf{0}^{(100)}$ & $\mathbf{0}^{(100)}$ & $\mathbf{0}^{(100)}$ \\
\hline $35(9.72 \%)$ & $\mathbf{0}^{(100)}$ & $\mathbf{0}^{(100)}$ & $\mathbf{0}^{(100)}$ \\
\hline $40(11.11 \%)$ & $\mathbf{0}^{(100)}$ & $\mathbf{0}^{(100)}$ & $\mathbf{0}^{(100)}$ \\
\hline $45(12.50 \%)$ & $\mathbf{0}^{(100)}$ & $\mathbf{0}^{(100)}$ & $\mathbf{0}^{(100)}$ \\
\hline $50(13.89 \%)$ & $\mathbf{0}^{(100)}$ & $\mathbf{0}^{(100)}$ & $\mathbf{0}^{(100)}$ \\
\hline $55(15.28 \%)$ & $\mathbf{0}^{(100)}$ & $\mathbf{0}^{(100)}$ & $\mathbf{0}^{(100)}$ \\
\hline $60(16.67 \%)$ & $\mathbf{0}^{(100)}$ & $\mathbf{0}^{(100)}$ & $\mathbf{0}^{(100)}$ \\
\hline $65(18.05 \%)$ & $\mathbf{0}^{(100)}$ & $\mathbf{0}^{(100)}$ & $\mathbf{0}^{(100)}$ \\
\hline $70(19.44 \%)$ & $1.56^{(91)}$ & $2.87^{(91)}$ & $\mathbf{0}^{(100)}$ \\
\hline $75(20.83 \%)$ & $2.81^{(98)}$ & $2.64^{(98)}$ & $0.07^{(99)}$ \\
\hline $80(22.22 \%)$ & $5.04^{(96)}$ & $5.01^{(96)}$ & $1.36^{(98)}$ \\
\hline $85(23.61 \%)$ & $3.34^{(97)}$ & $2.25^{(97)}$ & $1.19^{(98)}$ \\
\hline $90(25.00 \%)$ & $2.08^{(98)}$ & $1.94^{(98)}$ & $2.13^{(97)}$ \\
\hline $95(26.39 \%)$ & $6.38^{(92)}$ & $6.36^{(92)}$ & $5.28^{(92)}$ \\
\hline $100(27.78 \%)$ & $7.34^{(91)}$ & $6.86^{(91)}$ & $7.28^{(90)}$ \\
\hline \# Success & 1863 & 1863 & 1874 \\
\hline Critical Sparsity & $65(18.05 \%)$ & $65(18.05 \%)$ & $70(19.44 \%)$ \\
\hline Min m & $2.8 \mathrm{~S}$ & $2.8 \mathrm{~S}$ & $2.6 \mathrm{~S}$ \\
\hline
\end{tabular}

the average required time by $90.76 \%$ and $39.15 \%$ when compared to Method B and Method M, respectively.

Table 6.8 compares the speed of conventional methods with that of Algorithm ME1E2 $(\ell)$ in terms of processing time in seconds. The best results are bolded. According to the table, Algorithm ME1E2( $\ell$ ) needs less time to estimate a sparse solution compared to others up to a sparsity level of 65 (18.05\%). The second fastest method is SL0, which requires less time for sparse estimation for sparsity levels greater than $65(18.05 \%)$.

According to the results, MAX FS methods provide higher critical sparsity compared to the conventional methods and Algorithm ME1E2( $\ell)$ outperforms Method B and Method M. In general, using Algorithm ME1E2( $\ell)$ leads to the highest critical sparsity and number of successes compared to MAX FS and conventional sparse re- 
Table 6.5: Comparison of performances of the conventional algorithms using the average sparsity error (\%) in estimating the sparsity level of 100 ECG segments of length $n=360$ at $C R=50 \%$.

\begin{tabular}{|c|c|c|c|c|c|}
\hline \multirow{2}{*}{ S (sp \%) } & \multicolumn{5}{|c|}{$S_{e}(\%)$} \\
\hline & MP & PFP & OMP & SL0 & BP \\
\hline $10(2.78 \%)$ & $\mathbf{0}^{(100)}$ & $\mathbf{0}^{(100)}$ & $\mathbf{0}^{(100)}$ & $\mathbf{0}^{(100)}$ & $\mathbf{0}^{(100)}$ \\
\hline $15(4.17 \%)$ & $\mathbf{0}^{(100)}$ & $\mathbf{0}^{(100)}$ & $\mathbf{0}^{(100)}$ & $\mathbf{0}^{(100)}$ & $\mathbf{0}^{(100)}$ \\
\hline $20(5.56 \%)$ & $26.05^{(91)}$ & $\mathbf{0}^{(100)}$ & $\mathbf{0}^{(100)}$ & $\mathbf{0}^{(100)}$ & $\mathbf{0}^{(100)}$ \\
\hline $25(6.94 \%)$ & $29.80^{(92)}$ & $13.64^{(92)}$ & $\mathbf{0}^{(100)}$ & $\mathbf{0}^{(100)}$ & $\mathbf{0}^{(100)}$ \\
\hline $30(8.33 \%)$ & $52.90^{(82)}$ & $48.77^{(71)}$ & $6.47^{(97)}$ & $\mathbf{0}^{(100)}$ & $\mathbf{0}^{(100)}$ \\
\hline $35(9.72 \%)$ & $62.60^{(46)}$ & $88.57^{(37)}$ & $27.43^{(91)}$ & $\mathbf{0}^{(100)}$ & $\mathbf{0}^{(100)}$ \\
\hline $40(11.11 \%)$ & $115.52^{(7)}$ & $106.92^{(6)}$ & $22.35^{(74)}$ & $4.70^{(99)}$ & $\mathbf{0}^{(100)}$ \\
\hline $45(12.50 \%)$ & $102.73^{(2)}$ & $98.07^{(2)}$ & $10.73^{(61)}$ & $6.40^{(98)}$ & $\mathbf{0}^{(100)}$ \\
\hline $50(13.89 \%)$ & $84.02^{(0)}$ & $81.96^{(0)}$ & $9.46^{(34)}$ & $12.64^{(98)}$ & $\mathbf{0}^{(100)}$ \\
\hline $55(15.28 \%)$ & $67.85^{(0)}$ & $65.96^{(0)}$ & $20.47^{(10)}$ & $24.24^{(92)}$ & $\mathbf{0}^{(100)}$ \\
\hline $60(16.67 \%)$ & $54.08^{(0)}$ & $52.35^{(0)}$ & $26.58^{(0)}$ & $55.17^{(83)}$ & $5.32^{(98)}$ \\
\hline $65(18.05 \%)$ & $42.54^{(0)}$ & $40.63^{(0)}$ & $32.37^{(0)}$ & $96.80^{(65)}$ & $25.41^{(84)}$ \\
\hline $70(19.44 \%)$ & $32.90^{(0)}$ & $30.60^{(0)}$ & $37.67^{(0)}$ & $152.74^{(37)}$ & $69.36^{(49)}$ \\
\hline $75(20.83 \%)$ & $24.55^{(0)}$ & $21.85^{(0)}$ & $29.15^{(0)}$ & $189.92^{(18)}$ & $105.73^{(27)}$ \\
\hline $80(22.22 \%)$ & $17.12^{(0)}$ & $14.31^{(0)}$ & $21.70^{(0)}$ & $204.14^{(3)}$ & $116.34^{(8)}$ \\
\hline $85(23.61 \%)$ & $10.73^{(0)}$ & $7.82^{(0)}$ & $14.55^{(0)}$ & $194.25^{(0)}$ & $110.56^{(2)}$ \\
\hline $90(25.00 \%)$ & $4.68^{(0)}$ & $17.40^{(0)}$ & $13.83^{(0)}$ & $179.51^{(0)}$ & $99.47^{(2)}$ \\
\hline $95(26.39 \%)$ & $16.10^{(0)}$ & $10.98^{(0)}$ & $8.84^{(0)}$ & $166.29^{(0)}$ & $89.47^{(0)}$ \\
\hline $100(27.78 \%)$ & $10.57^{(0)}$ & $9.51^{(0)}$ & $12.74^{(0)}$ & $153.69^{(0)}$ & $80.00^{(0)}$ \\
\hline \# Success & 520 & 508 & 767 & 1193 & 1270 \\
\hline Critical Sparsity & $15(4.17 \%)$ & $20(5.56 \%)$ & $25(6.94 \%)$ & $35(9.72 \%)$ & $55(15.28 \%)$ \\
\hline Min $\mathrm{m}$ & $12 \mathrm{~S}$ & $9 \mathrm{~S}$ & $7.20 \mathrm{~S}$ & $5.14 \mathrm{~S}$ & $3.27 \mathrm{~S}$ \\
\hline
\end{tabular}

Table 6.6: Comparing average number of LPs and processing time in seconds of Method B, Method M, and the proposed Algorithm ME1E2( $\ell)$ for 100 ECG segments of length $n=360$ at $C R=50 \%$.

\begin{tabular}{|c|c|c|c|c|c|c|}
\hline \multirow{2}{*}{ S (sp \%) } & \multicolumn{2}{|c|}{ Method B } & \multicolumn{2}{|c|}{ Method M } & \multicolumn{2}{|c|}{$\operatorname{ME1E2}(\ell)$} \\
\hline & LPs & Sec & LPs & Sec & LPs & Sec \\
\hline $10(2.78 \%)$ & 19 & 0.03 & 1 & 0.001 & 1 & 0.001 \\
\hline $15(4.17 \%)$ & 28 & 0.03 & 1 & 0.001 & 1 & 0.001 \\
\hline $20(5.56 \%)$ & 37 & 0.03 & 1 & 0.001 & 1 & 0.001 \\
\hline $25(6.94 \%)$ & 48 & 0.04 & 1 & 0.001 & 1 & 0.001 \\
\hline $30(8.33 \%)$ & 59 & 0.05 & 1 & 0.001 & 1 & 0.001 \\
\hline $35(9.72 \%)$ & 69 & 0.06 & 1 & 0.001 & 1 & 0.001 \\
\hline $40(11.11 \%)$ & 79 & 0.07 & 1 & 0.001 & 1 & 0.001 \\
\hline $45(12.50 \%)$ & 89 & 0.09 & 1 & 0.001 & 1 & 0.001 \\
\hline $50(13.89 \%)$ & 99 & 0.10 & 1 & 0.001 & 1 & 0.001 \\
\hline $55(15.28 \%)$ & 109 & 0.12 & 1 & 0.001 & 1 & 0.001 \\
\hline $60(16.67 \%)$ & 119 & 0.14 & 120 & 0.14 & 1.84 & 0.002 \\
\hline $65(18.05 \%)$ & 129 & 0.16 & 128 & 0.16 & 7.56 & 0.01 \\
\hline $70(19.44 \%)$ & 139 & 0.18 & 139.10 & 0.18 & 22.6 & 0.03 \\
\hline $75(20.83 \%)$ & 151.12 & 0.21 & 151.15 & 0.21 & 32.84 & 0.04 \\
\hline $80(22.22 \%)$ & 163.08 & 0.24 & 164.01 & 0.24 & 43.06 & 0.06 \\
\hline $85(23.61 \%)$ & 172.92 & 0.28 & 172.94 & 0.28 & 47.06 & 0.06 \\
\hline $90(25.00 \%)$ & 182.55 & 0.31 & 183.55 & 0.31 & 49.01 & 0.07 \\
\hline $95(26.39 \%)$ & 197.60 & 0.34 & 197.71 & 0.34 & 51.38 & 0.07 \\
\hline $100(27.78 \%)$ & 211.99 & 0.34 & 212.09 & 0.34 & 52.54 & 0.07 \\
\hline
\end{tabular}


Table 6.7: Average percentage reductions in LPs solved and processing time by using Algorithm ME1E2 $(\ell)$ instead of Method B and Method M for 100 ECG segments of length $n=360$ at $C R=50 \%$.

\begin{tabular}{|c|c|c|c|c|}
\hline \multirow{2}{*}{$\mathrm{S}(\mathrm{sp} \%)$} & \multicolumn{2}{|c|}{ ME1E2 $(\ell)$ vs. B } & \multicolumn{2}{|c|}{ ME1E2( $\ell)$ vs. M } \\
\hline & LPs & Sec & LPs & Sec \\
\hline $10(2.78 \%)$ & 94.74 & 97.08 & 0 & 0 \\
\hline $15(4.17 \%)$ & 96.43 & 97.34 & 0 & 0 \\
\hline $20(5.56 \%)$ & 97.30 & 97.52 & 0 & 0 \\
\hline $25(6.94 \%)$ & 97.92 & 97.82 & 0 & 0 \\
\hline $30(8.33 \%)$ & 98.31 & 97.87 & 0 & 0 \\
\hline $35(9.72 \%)$ & 98.55 & 98.22 & 0 & 0 \\
\hline $40(11.11 \%)$ & 98.73 & 98.54 & 0 & 0 \\
\hline $45(12.50 \%)$ & 98.88 & 98.66 & 0 & 0 \\
\hline $50(13.89 \%)$ & 98.99 & 98.78 & 0 & 0 \\
\hline $55(15.28 \%)$ & 99.08 & 98.86 & 0 & 0 \\
\hline $60(16.67 \%)$ & 98.45 & 98.27 & 98.47 & 98.27 \\
\hline $65(18.05 \%)$ & 94.14 & 94.26 & 94.09 & 94.26 \\
\hline $70(19.44 \%)$ & 83.74 & 84.57 & 83.75 & 84.57 \\
\hline $75(20.83 \%)$ & 78.27 & 79.17 & 78.27 & 79.17 \\
\hline $80(22.22 \%)$ & 73.60 & 75.93 & 73.75 & 75.93 \\
\hline $85(23.61 \%)$ & 72.79 & 77.17 & 72.79 & 77.17 \\
\hline $90(25.00 \%)$ & 73.15 & 77.94 & 73.30 & 77.94 \\
\hline $95(26.39 \%)$ & 74.00 & 78.35 & 74.01 & 78.35 \\
\hline $100(27.78 \%)$ & 75.22 & 78.20 & 75.23 & 78.20 \\
\hline Average Imp. & 89.59 & 90.76 & 38.09 & 39.15 \\
\hline Max Imp & 99.08 & 98.86 & 98.47 & 98.27 \\
\hline
\end{tabular}

Table 6.8: Comparing average processing time in seconds of the conventional algorithms and the proposed Algorithm ME1E2( $\ell$ ) for 100 ECG segments of length $n=360$ at $C R=50 \%$.

\begin{tabular}{|c|c|c|c|c|c|c|}
\hline S (sp \%) & MP & PFP & OMP & SL0 & BP & ME1E2( $\ell \%)$ \\
\hline $10(2.78 \%)$ & 0.46 & 0.01 & 0.002 & 0.02 & 0.002 & 0.001 \\
\hline $15(4.17 \%)$ & 0.463 & 0.02 & 0.002 & 0.02 & 0.002 & 0.001 \\
\hline $20(5.56 \%)$ & 0.46 & 0.02 & 0.002 & 0.02 & 0.002 & 0.001 \\
\hline $25(6.94 \%)$ & 0.53 & 0.04 & 0.003 & 0.02 & 0.002 & 0.001 \\
\hline $30(8.33 \%)$ & 0.47 & 0.10 & 0.004 & 0.02 & 0.002 & 0.001 \\
\hline $35(9.72 \%)$ & 0.47 & 0.20 & 0.01 & 0.02 & 0.002 & 0.001 \\
\hline $40(11.11 \%)$ & 0.46 & 0.28 & 0.01 & 0.02 & 0.002 & 0.001 \\
\hline $45(12.50 \%)$ & 0.49 & 0.31 & 0.01 & 0.02 & 0.002 & 0.001 \\
\hline $50(13.89 \%)$ & 0.46 & 0.30 & 0.02 & 0.02 & 0.002 & 0.001 \\
\hline $55(15.28 \%)$ & 0.47 & 0.30 & 0.02 & 0.02 & 0.002 & 0.001 \\
\hline $60(16.67 \%)$ & 0.47 & 0.30 & 0.02 & 0.02 & 0.17 & 0.002 \\
\hline $65(18.05 \%)$ & 0.46 & 0.30 & 0.02 & 0.02 & 0.19 & 0.01 \\
\hline $70(19.44 \%)$ & 0.49 & 0.31 & 0.02 & 0.02 & 0.21 & 0.03 \\
\hline $75(20.83 \%)$ & 0.50 & 0.33 & 0.02 & 0.02 & 0.23 & 0.04 \\
\hline $80(22.22 \%)$ & 0.46 & 0.31 & 0.02 & 0.02 & 0.23 & 0.06 \\
\hline $85(23.61 \%)$ & 0.48 & 0.30 & 0.02 & 0.02 & 0.22 & 0.06 \\
\hline $90(25.00 \%)$ & 0.48 & 0.31 & 0.02 & 0.02 & 0.23 & 0.07 \\
\hline 95(26.39\%) & 0.48 & 0.31 & 0.02 & 0.02 & 0.22 & 0.07 \\
\hline $100(27.78 \%)$ & 0.46 & 0.30 & 0.02 & 0.02 & 0.22 & 0.07 \\
\hline
\end{tabular}

covery methods. Moreover, Algorithm ME1E2 $(\ell)$ is faster than other algorithms such as MP, PFP, OMP, SL0, BP, B, and M and requires almost 78.33\%, 71.11\%, 63.89\%, 
$49.42 \%, 20.49 \%, 7.14 \%$, and $7.14 \%$ fewer measurements for accurately approximating the input sparsity level, respectively.

\subsubsection{Quality of Recovered ECG Signals}

This section evaluates the quality of the recovered ECG signals using Algorithm ME1E2 $(\ell)$ and comparators considering different compression ratios. Speeds are also evaluated. The results are averaged over 47 ECG signals from the MIT-BIH Arrhythmia Database. The ECG signals are sparsified using DCT. Measurements are obtained for five different CRs: 10\%, 20\%, 30\%, 40\%, 50\%, and $60 \%$.

The quality of the recovered compressively sensed ECG signals is illustrated in Fig. 6.9 considering PRD as a function of CRs. PRD values in the range $0-2,2-9$, and larger than 9 are considered "very good", "good", and "not good", respectively [195]. For all CRs, amongst all the algorithms considered, the proposed Algorithm ME1E2 $(\ell)$ provides the smallest PRD. Based on [195], the quality of recovered signal of the proposed algorithm is considered as "very good" up to $C R=40 \%$. MP and PFP show almost the same performance and provide the largest PRD compared to the remaining methods.

In Fig. 6.10, recovered ECG signals (100m) using different sparse recovery methods for $C R=40 \%$ are compared with the original ECG signal. It is shown that the recovered ECG by the proposed method is almost identical to the input ECG signal.

Table 6.9 illustrates the speed of the MAX FS algorithms based on the number of LPs and the processing time in seconds averaged over 47 ECG signals of length 3600. The best results are shown in boldface. Table 6.9 shows the superior performance of Algorithm ME1E2 $(\ell)$ in improving the speed of Method B and Method M for all CRs. Moreover, Table 6.10 reports the percentage reduction in required LPs and processing 


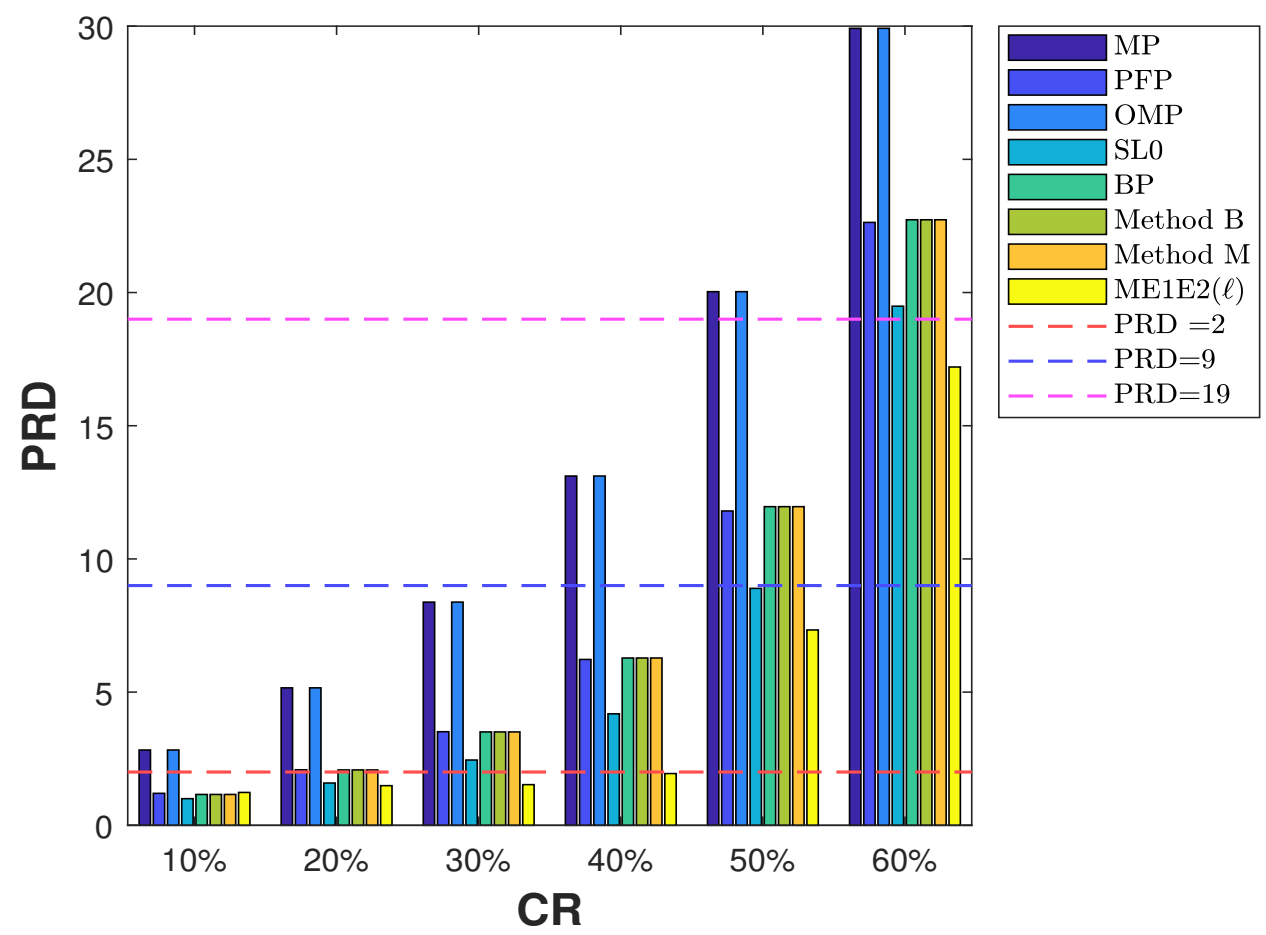

Figure 6.9: Comparing quality of the signal recovered by Method B and Algorithm ME1E2( $\ell$ ) using PRD, averaged over 47 ECG signals of length 3600 segmented into 10 frames of length $n=360$ at different $C R$ s.

time for Algorithm ME1E2( $\ell)$ when compared to Method B and Method M for 47 ECG signals and different CRs. At $\mathrm{CR}=40 \%$, Algorithm ME1E2 $(\ell)$ reduces the average required time by $80.64 \%$ and $80.31 \%$ when compared to that of Method B and Method M, respectively.

Table 6.9: Average algorithm speeds (number of LPs, Time in seconds) averaged over 47 ECG signals of length 3600 at different $C R$ s.

\begin{tabular}{|c|c|c|c|c|c|c|}
\hline \multirow{2}{*}{ CR } & \multicolumn{2}{|c|}{ B } & \multicolumn{2}{|c|}{ M } & \multicolumn{2}{c|}{ ME1E2 $(\ell)$} \\
\cline { 2 - 7 } & LPs & Secs & LPs & Secs & LPs & Secs \\
\hline $\mathbf{1 0 \%}$ & 6550 & 2930.07 & 6530 & 2917.89 & $\mathbf{5 9 6 . 0 8}$ & $\mathbf{3 6 5 . 5 2}$ \\
\hline $\mathbf{2 0 \%}$ & 5828 & 2172.02 & 5808 & 2156.18 & $\mathbf{6 1 0 . 9 8}$ & $\mathbf{3 1 9 . 8 3}$ \\
\hline $\mathbf{3 0 \%}$ & 5089 & 1465.41 & 5069 & 1452.04 & $\mathbf{6 2 6 . 2 5}$ & $\mathbf{2 5 5 . 4 5}$ \\
\hline $\mathbf{4 0 \%}$ & 4363 & 917.99 & 4343 & 902.71 & $\mathbf{6 3 7 . 5 1}$ & $\mathbf{1 7 7 . 7 3}$ \\
\hline $\mathbf{5 0 \%}$ & 3663 & 472.62 & 3643 & 469.46 & $\mathbf{6 4 1 . 2 9}$ & $\mathbf{1 0 7 . 1 1}$ \\
\hline $\mathbf{6 0 \%}$ & 2930 & 240.36 & 2910 & 237.82 & $\mathbf{6 2 2 . 6 8}$ & $\mathbf{5 9 . 8 1}$ \\
\hline
\end{tabular}

Table 6.11 compares the speed of the conventional sparse recovery methods with 

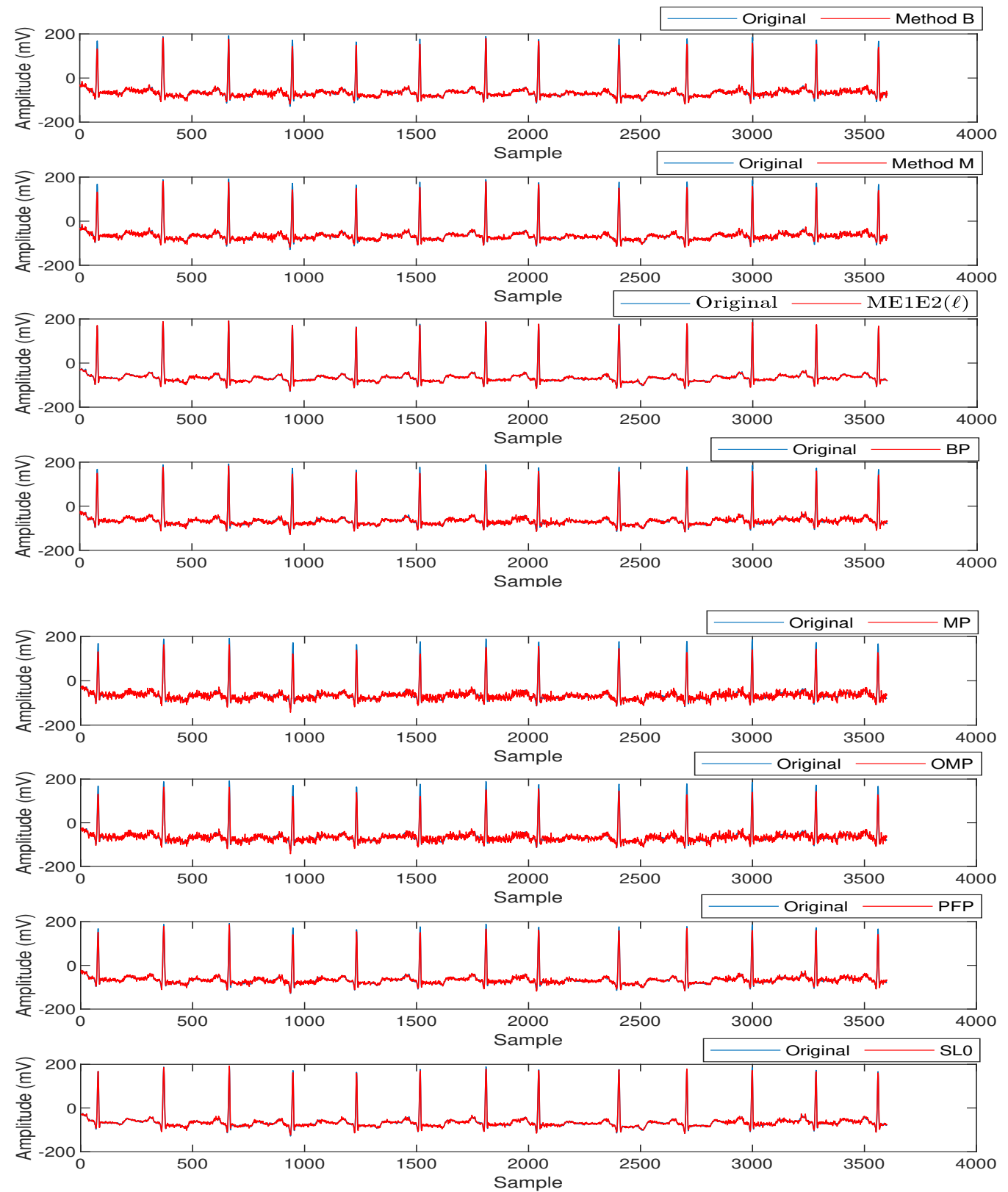

Figure 6.10: Comparing the input ECG signal and the signals recovered via the sparse recovery methods. The signals recovered by Methods B, M, ME1E2( $\ell)$, BP, MP, OMP, PFP, and SL0 have correlation coefficients of 0.983, 0.983, 0.997, 0.986, $0.954,0.986$, and 0.994 with the original signal $(\mathrm{CR}=50 \%, \mathrm{RNM})$.

that of Algorithm ME1E2 $(\ell)$. SL0 is the fastest method for all $C R \mathrm{~s}$. BP and Algorithm ME1E2 $(\ell)$ are the second and third fastest methods compared to others. 
Table 6.10: Average percent reduction in number of LPs and processing times for Algorithm ME1E2( $\ell$ ) vs. Methods B, and M for 47 ECG signals of length 3600 at different $C R \mathrm{~s}$.

\begin{tabular}{|c|c|c|c|c|}
\hline \multirow{2}{*}{ CR } & \multicolumn{2}{|c|}{ ME1E2( $\ell$ vs. B } & \multicolumn{2}{|c|}{ ME1E2 $(\ell)$ vs. M } \\
\cline { 2 - 5 } & LPs & Secs & LPs & Secs \\
\hline $\mathbf{1 0 \%}$ & 90.90 & 87.52 & 90.87 & 87.47 \\
\hline $\mathbf{2 0 \%}$ & 89.50 & 85.27 & 89.48 & 85.17 \\
\hline $\mathbf{3 0 \%}$ & 87.70 & 82.57 & 87.64 & 82.41 \\
\hline $\mathbf{4 0 \%}$ & 85.40 & 80.64 & 85.32 & 80.31 \\
\hline $\mathbf{5 0 \%}$ & 82.50 & 77.34 & 82.40 & 77.18 \\
\hline $\mathbf{6 0 \%}$ & 78.70 & 75.12 & 78.60 & 74.85 \\
\hline
\end{tabular}

Table 6.11: Average processing time (secs) required by Algorithm ME1E2 $\ell$ and conventional sparse recovery methods for 47 ECG signals of length 3600 at different $C R$ s.

\begin{tabular}{|c|c|c|c|c|c|c|}
\hline CR & MP & PFP & OMP & SL0 & BP & ME1E2 $(\ell)$ \\
\hline $\mathbf{1 0 \%}$ & 220.99 & 223.98 & 220.73 & $\mathbf{7 . 1 2}$ & 137.76 & 365.52 \\
\hline $\mathbf{2 0 \%}$ & 398.83 & 403.97 & 399.25 & $\mathbf{1 3 . 0 2}$ & 271.85 & 319.83 \\
\hline $\mathbf{3 0 \%}$ & 340.71 & 322.89 & 341.54 & $\mathbf{1 1 . 5 2}$ & 233.04 & 255.45 \\
\hline $\mathbf{4 0 \%}$ & 257.41 & 233.69 & 257.98 & $\mathbf{9 . 3 9}$ & 174.93 & 177.73 \\
\hline $\mathbf{5 0 \%}$ & 161.45 & 167.14 & 161.84 & $\mathbf{8 . 1 2}$ & 122.46 & 107.11 \\
\hline $\mathbf{6 0 \%}$ & 79.99 & 103.83 & 79.20 & $\mathbf{6 . 1 2}$ & 74.09 & 59.81 \\
\hline
\end{tabular}

\subsection{MAX FS for Sparse Recovery of Compres- sively Sensed Speech Signals}

Personal voice assistants (PVA) such as SIRI or Amazon Echo have become part of many households worldwide. PVAs may continuously monitor conversations in a house, transmit these conversations to the cloud for processing, and receive the processed information back. By 2023, the number of PVAs is expected to reach 8 million [203]. To reduce bandwidth consumption, there is a need to compress these signals.

Compression of speech has been explored since the $1930 \mathrm{~s}$ with the introduction of the Vocoder 204]. Since then, two types of speech compression techniques, namely, 
lossy and lossless compression techniques have evolved. Lossy techniques include differential coding, subband coding, $M P 3$, constrained energy lapped transform, and codec2 [204]. Lossless techniques include Recursive Least Mean Square (RLS), Least Mean Square (LMS), scalable lossless coding, and free lossless audio coding [205]. All of the aforementioned techniques are applied post sampling of the speech. As speech is sampled beyond Nyquist rate, sampling devices work at higher frequencies; therefore, no reduction in size, cost and power can be anticipated.

Compressive Sensing (CS) is well-suited to this task since the signal is compressed and coded at the same time. CS reduces the power consumption of the PVAs as the device samples much below the Nyquist frequency. The goal in compression techniques is to compress the data as much as possible in order to transmit/store less data. However, compression of the data cannot be done without considering the recovery aspect. For greater compression in CS, the critical sparsity of the sparse recovery method must be large enough to allow high quality signal recovery. As shown in Section 6.4.2.1, the critical sparsities of the existing and new MAX FS algorithms are higher than that of conventional CS sparse recovery methods in recovery of compressively sensed ECG signals. This section applies the proposed MAX FS algorithm in the sparse recovery phase of CS with the goal of providing higher critical sparsity to allow recovery of more highly compressed speech signals with greater quality.

Fig. 6.11 details a pipeline for recovering compressively sensed speech signals. The proposed pipeline is an example of how the proposed method can be used for recovery of compressively sensed speech in CS. The silent portions of a signal contain no useful information, so removing them decreases processing time. In the experiments, the word transcription information in the TIMIT dataset is used to identify the silent parts of the input. To circumvent the issue of the non-stationarity nature of speech signals, speech signals are typically sensed in short segments where stationary is 
assumed. In this work, $16 m s$ speech segments $(n=256)$ are considered since speech segment duration between $15 \mathrm{~ms}-35 \mathrm{~ms}$ is suggested for better speech intelligibility [206]. The performance of the sparse recovery algorithms is evaluated using RNM (refer to Section 6.4.1.1).

Let $\mathbf{f}_{S}$ be the vector containing only the terms corresponding to the $S$ largest values of $\mathbf{a}$, and let $\mathbf{a}_{S}$ be the vector $\mathbf{a}$ with the smallest $n-S$ terms set to zero. Based on [207], by using a proper sparsifying orthonormal basis $\boldsymbol{\Psi}$, we have $\left\|\mathbf{f}-\mathbf{f}_{S}\right\|_{2}=\left\|\mathbf{a}-\mathbf{a}_{\mathbf{S}}\right\|_{2}$ where $\mathbf{f}_{S}=\Psi \mathbf{a}_{S}$. When $\mathbf{a}$ is sparse or compressible, $\mathbf{a}$ is well estimated by using $\mathbf{a}_{\mathbf{S}}$ and, consequently, the error $\left\|\mathbf{f}-\mathbf{f}_{S}\right\|_{2}$ is small. Therefore, all except the $S$ largest components of the compressible signal a can be removed without much loss 78 . Based on [208], the best choice for sparsifying matrix for high quality speech recovery is DCT . Therefore, DCT is used for sparsifying the input signal in this work. Here, to obtain $\mathbf{a}_{\mathbf{S}}$, the DCT coefficients of each segment are sorted in descending order of magnitude; the DCT coefficients decay rapidly to zero if the signal is compressible. The $S$ largest coefficients are selected by thresholding. The threshold used here is 1.3 times the mean of all DCT coefficients in a segment and was fixed after examining over 100 different speech segments from the database used in this work.

The main contributions of this section are:

- Applying the proposed pipeline mentioned in Fig. 6.7 in recovery of real-world compressively sensed speech signals.

- Investigating the performance of state-of-the-art MAX FS algorithms (Method B and Method M) in recovery of the compressively sensed speech signals for the first time.

- Applying the new Algorithm ME1E2( $\ell)$ in recovery of the compressively sensed speech signals. 
$\overline{\text { Figure 6.11 Pipeline for compressing and recovering a compressively sensed speech }}$ signal.

Signal Acquisition:

Input: Input signal f.

Remove the silent parts of $\mathbf{f}$.

Divide $\mathbf{f}$, into segments of length $n$.

for each segment do:

Apply DCT.

Use only the $S$ largest DCT coefficients to generate an $S$-sparse vector $\mathbf{a}_{S}$ of length $n$.

Compress each sparsified segment using a random measurement matrix $\boldsymbol{\Phi}$ to obtain measurement vector $\mathbf{y}$.

end for

Signal Recovery:

Input: $\boldsymbol{\Phi}_{m \times n}$ and $\mathbf{y}_{m \times 1}$.

Select a CS recovery algorithm (e.g. Algorithm ME1E2( $\ell$ ) in Fig. 4.3).

for each segment do:

Apply a sparse recovery algorithm.

Take the inverse DCT of the estimated sparse segment.

end for

Concatenate segments to obtain recovered signal $\hat{\mathbf{f}}$.

Output: The recovered signal $\hat{\mathbf{f}}$. 
- Comparing the critical sparsity and recovered signal quality using the new and existing MAX FS algorithms against the state-of-the-art CS recovery methods for compressed speech segments.

- Comparing the speed of the newly developed Algorithm ME1E2( $\ell$ ) against the well-known MAX FS algorithms and conventional CS recovery methods in recovery of the compressively sensed speech signals.

In the remainder of this chapter, Section 6.5.1 and Section 6.5.2 provide experimental setup and empirical results for the recovery of compressively sensed real-world speech signals, respectively.

\subsubsection{Experimental Setup}

Since speech signals are not sparse by nature [209], sparsity varies greatly over time [210]; hence, compressing and recovery of non-sparse speech signals is a challenge. This section explains the experimental set up for recovery compressively sensed speech signals.

\subsubsection{Speech Database Description}

In the case of speech recovery, examples are drawn from the TIMIT database of speech samples that includes time-aligned orthographic, phonetic and word transcriptions and speech waveforms sampled at $16 \mathrm{kHz}[211]$. This well-known database has a total of 6300 sentences, 10 sentences spoken by each of 630 speakers, 438 male and 192 female, from 8 major dialect regions of the United States. 96 examples, 48 male and 48 female speakers, are used, covering all 8 dialect regions and all 3 types of sentences. The silent portions of a signal contain no useful information, so removing them decreases processing time. In our experiments, the silent parts of each 
input speech signal are removed based on the word transcription information, which provides the positions of non-silent parts of TIMIT speech signals.

\subsubsection{Performance Evaluation Metrics for Speech Recovery}

In addition to $S_{e}$ (refer to Eqn. (4.1)), the following metrics are used to evaluate the quality of the sparse recovery algorithms in recovery of compressively sensed speech signals.

- Relative Squared Error. The quality of the recovered speech signals is quantified based on Relative Squared Error (RSE) as mentioned in Eqn. 6.24).

$$
R S E=\frac{\sum_{j}\left(\hat{f}_{j}-f_{j}\right)^{2}}{\sum_{j}\left(f_{j}\right)^{2}} .
$$

- Perceptual Evaluation of Speech Quality (PESQ). A standardized algorithm which is called Perceptual Evaluation of Speech Quality (PESQ) 212 is used to assess the quality of speech. PESQ is recommended by the International Telecommunication Union (ITU) [213]. PESQ constructs a loudness spectrum by applying an auditory transform, which is a psychoacoustic model that projects the signals into a representation of perceived loudness in time and frequency 212]. Then the loudness spectra of the clean input signal are compared with that of the recovered signal to produce a single number in the range 1 (Bad) to 5 (Excellent) corresponding to the prediction of the perceptual mean opinion score.

\subsubsection{Software and Hardware}

Information about sparsifying, measurement matrix, comparators, software, and hardware is given in Section 6.4.1.1, Section 6.4.1.4, Section 6.4.1.5, and Section 
6.4.1.6, respectively.

\subsubsection{Results and Analysis}

This section focuses on recovery of compressively sensed speech signals. In Section 6.5.2.1, the critical sparsities of the different optimization techniques in compressively sensed speech recovery are investigated. Then, the quality of the recovered whole speech signal is evaluated in Section 6.5.2.2. The performances of the new Algorithm ME1E2 $(\ell)$ and existing MAX FS methods are compared with that of existing stateof-the-art algorithms in the results and discussion section.

\subsubsection{Critical Sparsity and Compression Degree of MAX FS Methods in Compressively Sensed Speech Recovery}

The concentration of the DCT coefficients in low and high frequency intervals affects the success of sparse recovery heuristics. In general, the lower frequency components are much stronger than the high frequency signal components. Also, lower frequency signal components represent information at a lower rate compared to high frequency signal components. The larger the number of DCT coefficients in low frequency intervals the higher the critical sparsity of a sparse recovery method. To show this clearly, two extreme cases are considered in the experiments: signals that have energy concentration in the "Low Frequency Region" $(\mathrm{LFR}$ ) and signals with energy concentration in the "High Frequency Region" (HFR).

A speech segment is LFR segment if the first 100 DCT coefficients (low frequencies) contribute more to the total energy in the signal than the rest. A speech segment is HFR segment if the components after the $100^{\text {th }}$ coefficient contribute significantly to the total energy of the signal (say 95\% of the total energy). 100 LFR and 100 HFR male and female speech segments examples of length $n=256$. are used for this set 
of experiments. Examples of LFR and HFR segments are shown in Fig. 6.12, The number of coefficients that constitute low frequency is decided after trial and error.
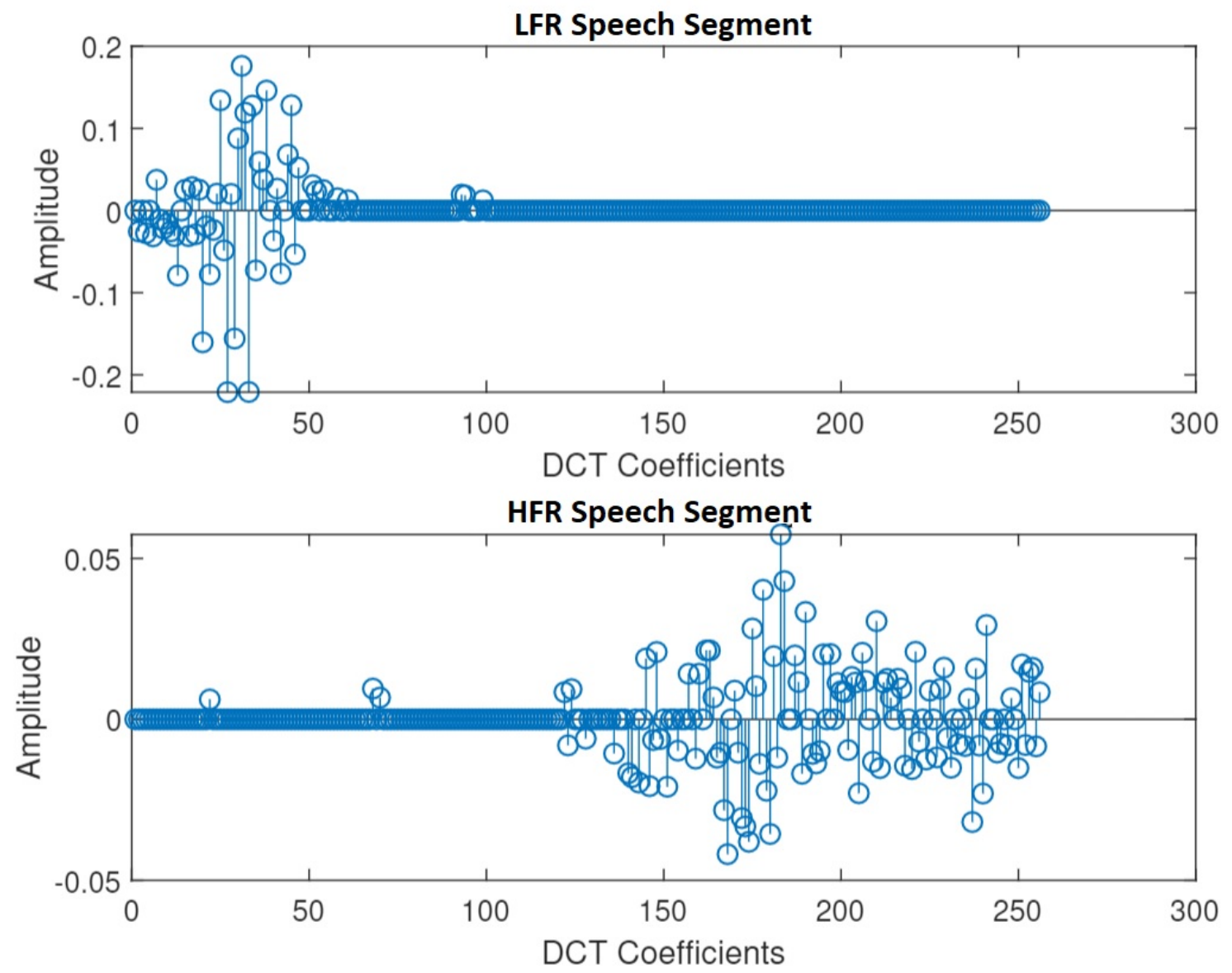

Figure 6.12: Example LFR and HFR segments of speech signal.

Table 6.12 and Table 6.13 illustrate the critical sparsity of different optimization algorithms for LFR and HFR speech segments for $C R=50 \%$ (the results of IRWLS and MP are excluded from this section due to their poor performance in sparse recovery).

The first column of the two tables (Table 6.12 and Table 6.13) represents the sparsity level $S$ and the percentage of input sparsity in the parentheses. The remaining columns indicate $S_{e}$ in percentage, ratio of the estimated sparsity over the input sparsity averaged over 100 segments. An algorithm succeeds in sparse recovery if it 
recovers a signal with sparsity $T=S$ in all 100 trials $\left(S_{e}=0 \%\right)$. The number of successes is shown in parentheses and complete success is bolded. The last three rows in Table 6.12 and Table 6.13 have the following meanings: "\# Success" expresses the total number of successes over each column, "Critical Sparsity" shows the maximum sparsity level that an algorithm is able to recover successfully in all 100 cases, and "Min $m$ " indicates the minimum number of measurements needed for each algorithm to succeed in estimating sparsity equal to $S$.

Except for PFP and BP, the critical sparsity of the sparse recovery methods for LFR is higher than for HFR. For both LFR and HFR segments the critical sparsity of MAX FS algorithms (Method B, Method M, and Algorithm ME1E2( $\ell$ )) is higher than that of conventional CS sparse recovery methods. Moreover, MAX FS algorithms require fewer measurements compared to the comparators. Method B and Method M provide more successes than Algorithm ME1E2( $\ell)$ and conventional CS sparse recovery methods.

Table 6.14 and Table 6.15 report the speed of the MAX FS algorithms in terms of number of LPs, "LPs", and processing time of the methods in seconds, "Sec". The best results in bold face. Based on the experimental results, Algorithm ME1E2( $\ell)$ needs fewer LPs and accordingly less time to estimate sparse solutions compared to Method B and Method M. Table 6.16 and Table 6.17 analyse the speed of the algorithms by indicating the percentage reduction in number of LPs and processing time for Algorithm ME1E2( $\ell)$ vs. Method B and Method M for each given sparsity level. The last two rows of Table 6.16 and Table 6.17 present the average improvement "Av-

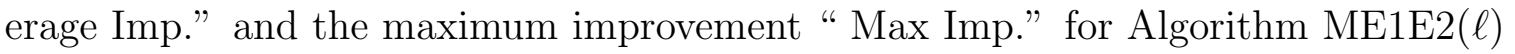
vs. the comparators. For both low pass and high pass segments, using Algorithm ME1E2 $(\ell)$ leads to a significant reduction in required number of LPs and processing time when compared to Method B and Method M. For instance, Algorithm ME1E2( $\ell)$ 
Table 6.12: Comparing performance of different sparse recovery algorithms using the average sparsity error (\%) in estimating the sparsity level of 100 speech LFR segments of length $n=256$ at $C R=50 \%$.

\begin{tabular}{|c|c|c|c|c|c|c|c|}
\hline S (sp \%) & PFP & OMP & SL0 & BP & B & $\mathbf{M}$ & ME1E2 $(\ell)$ \\
\hline $10(3.91 \%)$ & $\mathbf{0}^{(100)}$ & $\mathbf{0}^{(100)}$ & $\mathbf{0}^{(100)}$ & $\mathbf{0}^{(100)}$ & $\mathbf{0}^{(100)}$ & $\mathbf{0}^{(100)}$ & $\mathbf{0}^{(100)}$ \\
\hline $15(5.86 \%)$ & $\mathbf{0}^{(100)}$ & $\mathbf{0}^{(100)}$ & $\mathbf{0}^{(100)}$ & $\mathbf{0}^{(100)}$ & $\mathbf{0}^{(100)}$ & $\mathbf{0}^{(100)}$ & $\mathbf{0}^{(100)}$ \\
\hline $20(7.81 \%)$ & $\mathbf{0}^{(100)}$ & $\mathbf{0}^{(100)}$ & $\mathbf{0}^{(100)}$ & $\mathbf{0}^{(100)}$ & $\mathbf{0}^{(100)}$ & $\mathbf{0}^{(100)}$ & $\mathbf{0}^{(100)}$ \\
\hline $25(9.77 \%)$ & $\mathbf{0}^{(100)}$ & $\mathbf{0}^{(100)}$ & $\mathbf{0}^{(100)}$ & $\mathbf{0}^{(100)}$ & $\mathbf{0}^{(100)}$ & $\mathbf{0}^{(100)}$ & $\mathbf{0}^{(100)}$ \\
\hline $30(11.72 \%)$ & $\mathbf{0}^{(100)}$ & $\mathbf{0}^{(100)}$ & $\mathbf{0}^{(100)}$ & $\mathbf{0}^{(100)}$ & $\mathbf{0}^{(100)}$ & $\mathbf{0}^{(100)}$ & $\mathbf{0}^{(100)}$ \\
\hline $35(13.67 \%)$ & $\mathbf{0}^{(100)}$ & $\mathbf{0}^{(100)}$ & $\mathbf{0}^{(100)}$ & $\mathbf{0}^{(100)}$ & $\mathbf{0}^{(100)}$ & $\mathbf{0}^{(100)}$ & $\mathbf{0}^{(100)}$ \\
\hline $40(15.62 \%)$ & $\mathbf{0}^{(100)}$ & $\mathbf{0}^{(100)}$ & $29.33^{(96)}$ & $\mathbf{0}^{(100)}$ & $\mathbf{0}^{(100)}$ & $\mathbf{0}^{(100)}$ & $\mathbf{0}^{(100)}$ \\
\hline $45(17.58 \%)$ & $35.56^{(95)}$ & $11.63^{(98)}$ & $61.04^{(83)}$ & $36.89^{(98)}$ & $\mathbf{0}^{(100)}$ & $\mathbf{0}^{(100)}$ & $\mathbf{0}^{(100)}$ \\
\hline $50(19.53 \%)$ & $55.87^{(95)}$ & $15.00^{(97)}$ & $109.73^{(75)}$ & $67.20^{(94)}$ & $\mathbf{0}^{(100)}$ & $\mathbf{0}^{(100)}$ & $\mathbf{0}^{(100)}$ \\
\hline $55(21.48 \%)$ & $101.88^{(2)}$ & $33.21^{(92)}$ & $128.00^{(4)}$ & $118.73^{(87)}$ & $\mathbf{0}^{(100)}$ & $\mathbf{0}^{(100)}$ & $\mathbf{0}^{(100)}$ \\
\hline $60(23.44 \%)$ & $92.17^{(0)}$ & $73.00^{(9)}$ & $130.61^{(0)}$ & $105.44^{(3)}$ & $\mathbf{0}^{(100)}$ & $\mathbf{0}^{(100)}$ & $\mathbf{0}^{(100)}$ \\
\hline $65(25.39 \%)$ & $79.23^{(0)}$ & $75.85^{(4)}$ & $121.02^{(0)}$ & $96.56^{(2)}$ & $8.31^{(97)}$ & $8.29^{(96)}$ & $6.51^{(95)}$ \\
\hline $70(27.34 \%)$ & $67.05^{(0)}$ & $74.71^{(0)}$ & $109.43^{(0)}$ & $82.48^{(0)}$ & $28.62^{(88)}$ & $28.07^{(89)}$ & $20.33^{(90)}$ \\
\hline $75(29.30 \%)$ & $56.13^{(0)}$ & $63.07^{(0)}$ & $93.82^{(0)}$ & $70.49^{(0)}$ & $39.78^{(79)}$ & $38.92^{(80)}$ & $49.78^{(5)}$ \\
\hline $80(31.25 \%)$ & $46.08^{(0)}$ & $52.71^{(0)}$ & $84.54^{(0)}$ & $59.54^{(0)}$ & $45.08^{(4)}$ & $45.17^{(3)}$ & $50.87^{(2)}$ \\
\hline $85(33.20 \%)$ & $37.37^{(0)}$ & $43.61^{(0)}$ & $73.57^{(0)}$ & $50.16^{(0)}$ & $42.74^{(1)}$ & $42.62^{(1)}$ & $45.37^{(1)}$ \\
\hline $90(35.16 \%)$ & $30.07^{(0)}$ & $36.07^{(0)}$ & $63.78^{(0)}$ & $41.85^{(0)}$ & $35.63^{(0)}$ & $35.08^{(0)}$ & $37.70^{(0)}$ \\
\hline $95(37.11 \%)$ & $23.82^{(0)}$ & $28.91^{(0)}$ & $55.19^{(0)}$ & $34.28^{(0)}$ & $30.00^{(0)}$ & $30.03^{(0)}$ & $30.25^{(0)}$ \\
\hline $100(39.06 \%)$ & $17.37^{(0)}$ & $22.50^{(0)}$ & $46.80^{(0)}$ & $27.60^{(0)}$ & $22.70^{(0)}$ & $22.67^{(0)}$ & $24.30^{(0)}$ \\
\hline \# Success & 892 & 1000 & 858 & 984 & 1369 & 1369 & 1293 \\
\hline $\begin{array}{l}\text { Critical } \\
\text { Sparsity }\end{array}$ & $\begin{array}{c}40 \\
(11.11 \%)\end{array}$ & $\begin{array}{c}40 \\
(11.11 \%)\end{array}$ & $\begin{array}{c}35 \\
(9.72 \%)\end{array}$ & $\begin{array}{c}40 \\
(11.11 \%)\end{array}$ & $\begin{array}{c}60 \\
(16.67 \%)\end{array}$ & $\begin{array}{c}60 \\
(16.67 \%)\end{array}$ & $\begin{array}{c}60 \\
(16.67 \%)\end{array}$ \\
\hline Min m & $3.2 \mathrm{~S}$ & $3.2 \mathrm{~S}$ & $3.7 \mathrm{~S}$ & $3.2 \mathrm{~S}$ & $2.1 \mathrm{~S}$ & $2.1 \mathrm{~S}$ & $2.1 \mathrm{~S}$ \\
\hline
\end{tabular}

reduces the average required time by $77.82 \%$ and $43.45 \%$ when compared to Method $\mathrm{B}$ and Method $\mathrm{M}$ in Table 6.16, respectively.

Table 6.18 and Table 6.19 compare the speed of conventional methods with that of Algorithm ME1E2 $(\ell)$ in terms of processing time in seconds. The best results are in bold face. According to the tables, although Algorithm ME1E2 $(\ell)$ provides higher critical sparsity than the others, it needs more time to estimate a sparse solution compared to conventional sparse recovery methods. OMP and SL0 require less time to estimate the sparse solution compared to the others. In general, OMP is the fastest method up to sparsity level $45(17.58 \%)$ and SL0 requires less time for sparse estimation for sparsity levels greater than 50(19.53\%).

According to the results, MAX FS methods provide higher critical sparsity compared to the conventional methods and Algorithm ME1E2 $(\ell)$ reduces the processing 
Table 6.13: Comparing performance of different sparse recovery algorithms using the average sparsity error (\%) in estimating the sparsity level of 100 speech HFR segments of length $n=256$ at $C R=50 \%$.

\begin{tabular}{|c|c|c|c|c|c|c|c|}
\hline $\mathrm{S}(\mathrm{sp} \%)$ & PFP & OMP & SL0 & BP & B & $\mathbf{M}$ & $\operatorname{ME1E2}(\ell)$ \\
\hline $10(3.91 \%)$ & $\mathbf{0}^{(100)}$ & $\mathbf{0}^{(100)}$ & $\mathbf{0}^{(100)}$ & $\mathbf{0}^{(100)}$ & $\mathbf{0}^{(100)}$ & $\mathbf{0}^{(100)}$ & $\mathbf{0}^{(100)}$ \\
\hline $15(5.86 \%)$ & $\mathbf{0}^{(100)}$ & $\mathbf{0}^{(100)}$ & $\mathbf{0}^{(100)}$ & $\mathbf{0}^{(100)}$ & $\mathbf{0}^{(100)}$ & $\mathbf{0}^{(100)}$ & $\mathbf{0}^{(100)}$ \\
\hline $20(7.81 \%)$ & $\mathbf{0}^{(100)}$ & $\mathbf{0}^{(100)}$ & $\mathbf{0}^{(100)}$ & $\mathbf{0}^{(100)}$ & $\mathbf{0}^{(100)}$ & $\mathbf{0}^{(100)}$ & $\mathbf{0}^{(100)}$ \\
\hline $25(9.77 \%)$ & $\mathbf{0}^{(100)}$ & $\mathbf{0}^{(100)}$ & $\mathbf{0}^{(100)}$ & $\mathbf{0}^{(100)}$ & $\mathbf{0}^{(100)}$ & $\mathbf{0}^{(100)}$ & $\mathbf{0}^{(100)}$ \\
\hline $30(11.72 \%)$ & $\mathbf{0}^{(100)}$ & $\mathbf{0}^{(100)}$ & $18.25^{(99)}$ & $\mathbf{0}^{(100)}$ & $\mathbf{0}^{(100)}$ & $\mathbf{0}^{(100)}$ & $\mathbf{0}^{(100)}$ \\
\hline $35(13.67 \%)$ & $\mathbf{0}^{(100)}$ & $12.52^{(99)}$ & $40.24^{(97)}$ & $\mathbf{0}^{(100)}$ & $\mathbf{0}^{(100)}$ & $\mathbf{0}^{(100)}$ & $\mathbf{0}^{(100)}$ \\
\hline $40(15.62 \%)$ & $\mathbf{0}^{(100)}$ & $20.12^{(98)}$ & $131.67^{(9)}$ & $\mathbf{0}^{(100)}$ & $\mathbf{0}^{(100)}$ & $\mathbf{0}^{(100)}$ & $\mathbf{0}^{(100)}$ \\
\hline $45(17.58 \%)$ & $31.50^{(97)}$ & $75.66^{(91)}$ & $206.67^{(2)}$ & $35.03^{(97)}$ & $\mathbf{0}^{(100)}$ & $\mathbf{0}^{(100)}$ & $\mathbf{0}^{(100)}$ \\
\hline $50(19.53 \%)$ & $88.19^{(8)}$ & $135.33^{(2)}$ & $212.19^{(0)}$ & $96.38^{(8)}$ & $13.62^{(98)}$ & $13.12^{(98)}$ & $15.24^{(97)}$ \\
\hline $55(21.48 \%)$ & $111.25^{(2)}$ & $126.49^{(0)}$ & $187.01^{(0)}$ & $119.83^{(2)}$ & $18.35^{(97)}$ & $18.45^{(97)}$ & $37.58^{(92)}$ \\
\hline $60(23.44 \%)$ & $98.89^{(1)}$ & $108.81^{(0)}$ & $164.92^{(0)}$ & $107.86^{(1)}$ & $66.51^{(8)}$ & $65.89^{(8)}$ & $73.33^{(5)}$ \\
\hline $65(25.39 \%)$ & $87.98^{(0)}$ & $92.97^{(0)}$ & $144.54^{(0)}$ & $96.85^{(0)}$ & $88.42^{(1)}$ & $89.01^{(1)}$ & $84.69^{(2)}$ \\
\hline $70(27.34 \%)$ & $75.24^{(0)}$ & $78.43^{(0)}$ & $130.07^{(0)}$ & $82.79^{(0)}$ & $78.98^{(0)}$ & $79.35^{(0)}$ & $79.80^{(0)}$ \\
\hline $75(29.30 \%)$ & $63.24^{(0)}$ & $66.98^{(0)}$ & $114.22^{(0)}$ & $70.60^{(0)}$ & $65.52^{(0)}$ & $65.02^{(0)}$ & $66.86^{(0)}$ \\
\hline $80(31.25 \%)$ & $53.87^{(0)}$ & $56.55^{(0)}$ & $98.57^{(0)}$ & $59.88^{(0)}$ & $56.37^{(0)}$ & $56.81^{(0)}$ & $56.43^{(0)}$ \\
\hline $85(33.20 \%)$ & $45.04^{(0)}$ & $47.34^{(0)}$ & $87.06^{(0)}$ & $50.53^{(0)}$ & $47.34^{(0)}$ & $47.24^{(0)}$ & $48.18^{(0)}$ \\
\hline $90(35.16 \%)$ & $37.09^{(0)}$ & $39.21^{(0)}$ & $78.84^{(0)}$ & $42.12^{(0)}$ & $40.53^{(0)}$ & $40.35^{(0)}$ & $39.68^{(0)}$ \\
\hline $95(37.11 \%)$ & $28.97^{(0)}$ & $31.88^{(0)}$ & $69.57^{(0)}$ & $34.69^{(0)}$ & $31.73^{(0)}$ & $32.43^{(0)}$ & $32.73^{(0)}$ \\
\hline $100(39.06 \%)$ & $23.33^{(0)}$ & $25.19^{(0)}$ & $61.90^{(0)}$ & $28.00^{(0)}$ & $25.95^{(0)}$ & $21.95^{(0)}$ & $26.24^{(0)}$ \\
\hline \# Success & 808 & 790 & 607 & 808 & 1004 & 1004 & 996 \\
\hline $\begin{array}{l}\text { Critical } \\
\text { Sparsity }\end{array}$ & $\begin{array}{c}40 \\
(15.62 \%)\end{array}$ & $\begin{array}{c}30 \\
(11.72 \%)\end{array}$ & $\begin{array}{c}25 \\
(9.77 \%)\end{array}$ & $\begin{array}{c}40 \\
(11.11 \%)\end{array}$ & $\begin{array}{c}45 \\
(17.58 \%)\end{array}$ & $\begin{array}{c}45 \\
(17.58 \%)\end{array}$ & $\begin{array}{c}45 \\
(17.58 \%)\end{array}$ \\
\hline Min $\mathrm{m}$ & $3.2 \mathrm{~S}$ & $4.3 \mathrm{~S}$ & $5.1 \mathrm{~S}$ & $3.2 \mathrm{~S}$ & $2.8 \mathrm{~S}$ & $2.8 \mathrm{~S}$ & $2.8 \mathrm{~S}$ \\
\hline
\end{tabular}

Table 6.14: Comparing average number of LPs and processing time in seconds of Method B, Method M, and Algorithm ME1E2( $\ell$ ) for 100 speech LFR segments of length $n=256$ at $C R=50 \%$.

\begin{tabular}{|c|c|c|c|c|c|c|}
\hline \multirow{2}{*}{$\mathrm{S}(\mathrm{sp} \%)$} & \multicolumn{2}{|c|}{ Method B } & \multicolumn{2}{|c|}{ Method M } & \multicolumn{2}{|c|}{ ME1E2 $(\ell)$} \\
\hline & LPs & Sec & LPs & Sec & LPs & Sec \\
\hline $10(3.91 \%)$ & 10 & 0.41 & 1 & 0.05 & 1 & 0.05 \\
\hline $15(5.86 \%)$ & 15 & 0.61 & 1 & 0.05 & $\mathbf{1}$ & 0.05 \\
\hline $20(7.81 \%)$ & 20 & 0.81 & 1 & 0.05 & 1 & 0.05 \\
\hline $25(9.77 \%)$ & 25 & 0.93 & 1 & 0.05 & 1 & 0.05 \\
\hline $30(11.72 \%)$ & 30 & 1.16 & 1 & 0.05 & 1 & 0.05 \\
\hline $35(13.67 \%)$ & 35 & 1.45 & 1 & 0.06 & 1 & 0.06 \\
\hline $40(15.62 \%)$ & 40 & 1.70 & $\mathbf{1}$ & 0.11 & $\mathbf{1}$ & 0.11 \\
\hline $45(17.58 \%)$ & 45 & 2.01 & 45 & 2.01 & 4.33 & 0.25 \\
\hline $50(19.53 \%)$ & 50 & 2.36 & 50 & 2.36 & 8.37 & 0.49 \\
\hline $55(21.48 \%)$ & 55 & 2.78 & 55 & 2.78 & 14.53 & 0.86 \\
\hline $60(23.44 \%)$ & 60 & 3.35 & 60 & 3.35 & 18.67 & 1.12 \\
\hline $65(25.39 \%)$ & 70.67 & 3.97 & 70.57 & 3.97 & 20.87 & 1.30 \\
\hline $70(27.34 \%)$ & 91.27 & 5.23 & 90.92 & 5.23 & 25.70 & 1.62 \\
\hline $75(29.30 \%)$ & 106.17 & 6.02 & 106.09 & 6.12 & 35.00 & 2.35 \\
\hline $80(31.25 \%)$ & 117.67 & 6.66 & 117.51 & 6.66 & 36.70 & 2.61 \\
\hline $85(33.20 \%)$ & 123.2 & 7.04 & 123.02 & 7.04 & 38.10 & 2.46 \\
\hline $90(35.16 \%)$ & 124.03 & 7.09 & 124.11 & 7.09 & 38.87 & 2.46 \\
\hline $95(37.11 \%)$ & 125.20 & 7.17 & 125.12 & 7.17 & 38.90 & 2.31 \\
\hline $100(39.06 \%)$ & 124.13 & 7.04 & 124.16 & 7.02 & 39.43 & 2.33 \\
\hline
\end{tabular}


Table 6.15: Comparing average number of LPs and processing time in seconds of Method B, Method M, and the proposed Algorithm ME1E2( $\ell$ ) for 100 speech HFR segments of length $n=256$ at $C R=50 \%$.

\begin{tabular}{|c|c|c|c|c|c|c|}
\hline \multirow{2}{*}{ S (sp \%) } & \multicolumn{2}{|c|}{ Method B } & \multicolumn{2}{|c|}{ Method M } & \multicolumn{2}{|c|}{$\operatorname{ME1E2}(\ell)$} \\
\hline & LPs & Sec & LPs & Sec & LPs & Sec \\
\hline $10(3.91 \%)$ & 10 & 0.36 & 1 & 0.04 & 1 & 0.04 \\
\hline $15(5.86 \%)$ & 15 & 0.54 & 1 & 0.04 & 1 & 0.04 \\
\hline $20(7.81 \%)$ & 20 & 0.74 & 1 & 0.04 & 1 & 0.04 \\
\hline $25(9.77 \%)$ & 25 & 0.97 & 1 & 0.05 & 1 & 0.05 \\
\hline $30(11.72 \%)$ & 30 & 1.21 & 1 & 0.05 & 1 & 0.05 \\
\hline $35(13.67 \%)$ & 35 & 1.46 & 1 & 0.05 & 1 & 0.05 \\
\hline $40(15.62 \%)$ & 40 & 1.77 & 1 & 0.10 & 1 & 0.10 \\
\hline $45(17.58 \%)$ & 45 & 2.01 & 45 & 2.01 & 3.14 & 0.17 \\
\hline $50(19.53 \%)$ & 60.11 & 2.84 & 60.11 & 2.84 & 10.71 & 0.64 \\
\hline $55(21.48 \%)$ & 67.54 & 3.45 & 67.54 & 3.45 & 20.24 & 1.20 \\
\hline $60(23.44 \%)$ & 70.35 & 5.62 & 70.35 & 5.62 & 32.05 & 1.97 \\
\hline $65(25.39 \%)$ & 70.67 & 6.85 & 70.67 & 6.85 & 33.95 & 2.07 \\
\hline $70(27.34 \%)$ & 91.27 & 7.01 & 91.35 & 7.01 & 38.01 & 2.33 \\
\hline $75(29.30 \%)$ & 106.17 & 6.98 & 106.17 & 6.98 & 36.90 & 2.26 \\
\hline $80(31.25 \%)$ & 117.67 & 7.02 & 117.87 & 7.02 & 38.90 & 2.68 \\
\hline $85(33.20 \%)$ & 123.20 & 7.01 & 123.25 & 7.01 & 38.82 & 2.60 \\
\hline $90(35.16 \%)$ & 124.03 & 7.12 & 124.03 & 7.12 & 38.09 & 2.35 \\
\hline $95(37.11 \%)$ & 125.2 & 6.99 & 125.27 & 7.02 & $\mathbf{3 7 . 5 7}$ & 2.32 \\
\hline $100(39.06 \%)$ & 124.13 & 7.04 & 124.41 & 7.03 & 37.86 & 2.34 \\
\hline
\end{tabular}

Table 6.16: Average percentage reductions in LPs solved and processing time by using Algorithm ME1E2 $(\ell)$ instead of Method B and Method M for 100 speech LFR segments of length $n=256$ at $C R=50 \%$.

\begin{tabular}{|c|c|c|c|c|}
\hline \multirow{2}{*}{$\mathrm{S}(\mathrm{sp} \%)$} & \multicolumn{2}{|c|}{ ME1E2( $\ell)$ vs. B } & \multicolumn{2}{|c|}{ ME1E2 $(\ell)$ vs. M } \\
\hline & LPs & Sec & LPs & Sec \\
\hline $10(2.78 \%)$ & 90.00 & 88.81 & 0 & 0 \\
\hline $15(4.17 \%)$ & 93.33 & 92.31 & 0 & 0 \\
\hline $20(5.56 \%)$ & 95.00 & 93.81 & 0 & 0 \\
\hline $25(6.94 \%)$ & 96.00 & 94.29 & 0 & 0 \\
\hline $30(8.33 \%)$ & 96.67 & 95.27 & 0 & 0 \\
\hline $35(9.72 \%)$ & 97.14 & 95.86 & 0 & 0 \\
\hline $40(11.11 \%)$ & 97.50 & 93.23 & 0 & 0 \\
\hline $45(12.50 \%)$ & 90.38 & 87.38 & 90.38 & 87.38 \\
\hline $50(13.89 \%)$ & 83.26 & 79.21 & 83.26 & 79.21 \\
\hline $55(15.28 \%)$ & 73.58 & 68.88 & 73.58 & 68.92 \\
\hline $60(16.67 \%)$ & 68.88 & 66.66 & 68.88 & 66.66 \\
\hline $65(18.05 \%)$ & 70.47 & 67.25 & 70.43 & 67.24 \\
\hline $70(19.44 \%)$ & 71.84 & 68.95 & 71.73 & 68.95 \\
\hline $75(20.83 \%)$ & 67.03 & 60.89 & 67.01 & 61.53 \\
\hline $80(22.22 \%)$ & 68.81 & 60.80 & 68.77 & 60.8 \\
\hline $85(23.61 \%)$ & 69.07 & 65.05 & 69.03 & 65.05 \\
\hline $90(25.00 \%)$ & 68.66 & 65.32 & 68.68 & 65.32 \\
\hline $95(26.39 \%)$ & 68.93 & 67.77 & 68.91 & 67.78 \\
\hline $100(27.78 \%)$ & 68.23 & 66.88 & 68.24 & 66.79 \\
\hline Average Imp. & 80.78 & 77.82 & 45.73 & 43.45 \\
\hline Max Imp & 97.50 & 95.86 & 90.38 & 87.38 \\
\hline
\end{tabular}

time of Method B and Method M. Algorithm ME1E2( $\ell)$ has more successes compared to conventional sparse recovery methods while requiring less time to recover 
Table 6.17: Average percentage reductions in LPs solved and processing time by using Algorithm ME1E2( $\ell$ ) instead of Method B and Method M for 100 speech HFR segments of length $n=256$ at $C R=50 \%$.

\begin{tabular}{|c|c|c|c|c|}
\hline \multirow{2}{*}{$\mathrm{S}(\mathrm{sp} \%)$} & \multicolumn{2}{|c|}{ ME1E2 $(\ell)$ vs. B } & \multicolumn{2}{|c|}{ ME1E2( $\ell)$ vs. M } \\
\hline & LPs & Sec & LPs & Sec \\
\hline $10(3.91 \%)$ & 90.00 & 89.17 & 0 & 0 \\
\hline $15(5.86 \%)$ & 93.33 & 92.42 & 0 & 0 \\
\hline $20(7.81 \%)$ & 95.00 & 94.20 & 0 & 0 \\
\hline $25(9.77 \%)$ & 96.00 & 95.27 & 0 & 0 \\
\hline $30(11.72 \%)$ & 96.67 & 95.86 & 0 & 0 \\
\hline $35(13.67 \%)$ & 97.14 & 96.30 & 0 & 0 \\
\hline $40(15.62 \%)$ & 97.50 & 94.36 & 0 & 0 \\
\hline $45(17.58 \%)$ & 93.02 & 91.28 & 93.02 & 91.28 \\
\hline $50(19.53 \%)$ & 82.18 & 77.51 & 82.18 & 77.51 \\
\hline $55(21.48 \%)$ & 70.03 & 65.24 & 70.03 & 65.24 \\
\hline $60(23.44 \%)$ & 54.44 & 64.89 & 54.44 & 64.89 \\
\hline $65(25.39 \%)$ & 51.96 & 69.79 & 51.96 & 69.79 \\
\hline $70(27.34 \%)$ & 58.35 & 66.79 & 58.39 & 66.81 \\
\hline $75(29.30 \%)$ & 65.24 & 67.67 & 65.24 & 67.67 \\
\hline $80(31.25 \%)$ & 66.94 & 61.83 & 66.99 & 61.86 \\
\hline $85(33.20 \%)$ & 68.49 & 62.86 & 68.50 & 62.89 \\
\hline $90(35.16 \%)$ & 69.29 & 66.97 & 69.29 & 66.97 \\
\hline $95(37.11 \%)$ & 69.99 & 66.83 & 70.01 & 66.92 \\
\hline $100(39.06 \%)$ & 69.50 & 66.69 & 69.57 & 66.68 \\
\hline Average Imp. & 78.16 & 78.21 & 43.14 & 43.61 \\
\hline Max Imp & 97.50 & 96.30 & 93.02 & 91.28 \\
\hline
\end{tabular}

Table 6.18: Comparing average processing time in seconds of the conventional algorithms and the proposed Algorithm ME1E2 $(\ell)$ for 100 LFR speech segments of length $n=256$ at $C R=50 \%$.

\begin{tabular}{|c|c|c|c|c|c|}
\hline S (sp \%) & PFP & OMP & SL0 & BP & ME1E2 $(\ell)$ \\
\hline $10(3.91 \%)$ & 0.01 & 0.002 & 0.01 & 0.04 & 0.05 \\
\hline $15(5.86 \%)$ & 0.01 & 0.001 & 0.01 & 0.04 & 0.05 \\
\hline $20(7.81 \%)$ & 0.01 & 0.002 & 0.01 & 0.04 & 0.05 \\
\hline $25(9.77 \%)$ & 0.01 & 0.002 & 0.01 & 0.04 & 0.05 \\
\hline $30(11.72 \%)$ & 0.02 & 0.003 & 0.005 & 0.05 & 0.05 \\
\hline $35(13.67 \%)$ & 0.03 & 0.003 & 0.01 & 0.05 & 0.06 \\
\hline $40(15.62 \%)$ & 0.04 & 0.003 & 0.01 & 0.05 & 0.11 \\
\hline $45(17.58 \%)$ & 0.06 & 0.005 & 0.01 & 0.06 & 0.25 \\
\hline $50(19.53 \%)$ & 0.07 & 0.01 & 0.01 & 0.06 & 0.49 \\
\hline $55(21.48 \%)$ & 0.08 & 0.01 & 0.01 & 0.07 & 0.86 \\
\hline $60(23.44 \%)$ & 0.08 & 0.01 & 0.01 & 0.07 & 1.12 \\
\hline $65(25.39 \%)$ & 0.08 & 0.01 & 0.005 & 0.07 & 1.30 \\
\hline $70(27.34 \%)$ & 0.08 & 0.01 & 0.01 & 0.071 & 1.62 \\
\hline $75(29.30 \%)$ & 0.08 & 0.01 & 0.005 & 0.07 & 2.35 \\
\hline $80(31.25 \%)$ & 0.08 & 0.01 & 0.005 & 0.07 & 2.61 \\
\hline $85(33.20 \%)$ & 0.08 & 0.01 & 0.01 & 0.07 & 2.46 \\
\hline $90(35.16 \%)$ & 0.08 & 0.01 & 0.01 & 0.07 & 2.46 \\
\hline $95(37.11 \%)$ & 0.08 & 0.01 & 0.01 & 0.071 & 2.31 \\
\hline $100(39.06 \%)$ & 0.08 & 0.01 & 0.01 & 0.069 & 2.33 \\
\hline
\end{tabular}

when compared to well-known MAX FS methods. To illustrate, Algorithm ME1E2( $\ell)$ requires only $M>2 S$ for accurate sparse estimation for LFR speech segments, a re- 
Table 6.19: Comparing average processing time in seconds of the conventional algorithms and the proposed Algorithm ME1E2 $(\ell)$ for $100 \mathrm{HFR}$ speech segments of length $n=256$ at $C R=50 \%$.

\begin{tabular}{|c|c|c|c|c|c|}
\hline S (sp \%) & PFP & OMP & SL0 & BP & ME1E2( $\ell \%)$ \\
\hline $10(3.91 \%)$ & 0.01 & 0.004 & 0.01 & 0.04 & 0.04 \\
\hline $15(5.86 \%)$ & 0.01 & 0.001 & 0.005 & 0.04 & 0.04 \\
\hline $20(7.81 \%)$ & 0.01 & 0.001 & 0.01 & 0.04 & 0.04 \\
\hline $25(9.77 \%)$ & 0.01 & 0.002 & 0.005 & 0.04 & 0.05 \\
\hline $30(11.72 \%)$ & 0.02 & 0.003 & 0.01 & 0.05 & 0.05 \\
\hline $35(13.67 \%)$ & 0.03 & 0.004 & 0.005 & 0.05 & 0.05 \\
\hline $40(15.62 \%)$ & 0.04 & 0.005 & 0.01 & 0.05 & 0.10 \\
\hline $45(17.58 \%)$ & 0.05 & 0.01 & 0.01 & 0.06 & 0.17 \\
\hline $50(19.53 \%)$ & 0.07 & 0.01 & 0.005 & 0.06 & 0.64 \\
\hline $55(21.48 \%)$ & 0.08 & 0.01 & 0.005 & 0.06 & 1.20 \\
\hline $60(23.44 \%)$ & 0.08 & 0.01 & 0.005 & 0.06 & 1.97 \\
\hline $65(25.39 \%)$ & 0.09 & 0.01 & 0.005 & 0.06 & 2.07 \\
\hline $70(27.34 \%)$ & 0.08 & 0.01 & 0.005 & 0.07 & 2.32 \\
\hline $75(29.30 \%)$ & 0.08 & 0.01 & 0.005 & 0.07 & 2.26 \\
\hline $80(31.25 \%)$ & 0.08 & 0.01 & 0.005 & 0.07 & 2.68 \\
\hline $85(33.20 \%)$ & 0.08 & 0.01 & 0.005 & 0.06 & 2.60 \\
\hline $90(35.16 \%)$ & 0.08 & 0.01 & 0.005 & 0.07 & 2.35 \\
\hline $95(37.11 \%)$ & 0.08 & 0.01 & 0.005 & 0.07 & 2.32 \\
\hline $100(39.06)$ & 0.08 & 0.01 & 0.005 & 0.07 & 2.34 \\
\hline
\end{tabular}

duction of $34.00 \%, 34.00 \%, 47.62 \%, 34.00 \%$ in the number of measurements required for recovery when compared with PFP, OMP, SL0, and BP respectively. In addition, Algorithm ME1E2( $\ell$ ) improves the speed of Method B and Method M by about $77 \%$ and $43 \%$ on average.

\subsubsection{Quality of Recovered Speech Signals}

Each speech signal is segmented into frames of length 256. After taking the DCT of each segment, the $S$ largest coefficients are selected by thresholding, where the threshold in each segment is 1.3 times the mean of all of its DCT coefficients. The sparsity of the entire speech signal is the sum of the sparsities of all of its segments. The speech inputs are compressed at $\mathrm{CR}=50 \%$. To recover the complete speech signal, all recovered segments are concatenated after taking the inverse DCT.

The 96 recovered signals are evaluated using the Relative Squared Error (RSE) as plotted in Fig. 6.13. The RSEs for the 96 speech signals of different lengths are 
shown as box-and-whisker plots with the median RSE as the central mark in the box and the $25 t h$ and $75 t h$ percentiles as the box boundaries. The whiskers extend to the most extreme RSE that are not considered outliers, and the outliers are plotted using the ${ }^{\prime}+{ }^{\prime}$ symbol. The RSEs for the MAX FS methods are very small. They provide higher fidelity recovered signals even though their solutions are sparser than those of the other algorithms as discussed in the previous section.

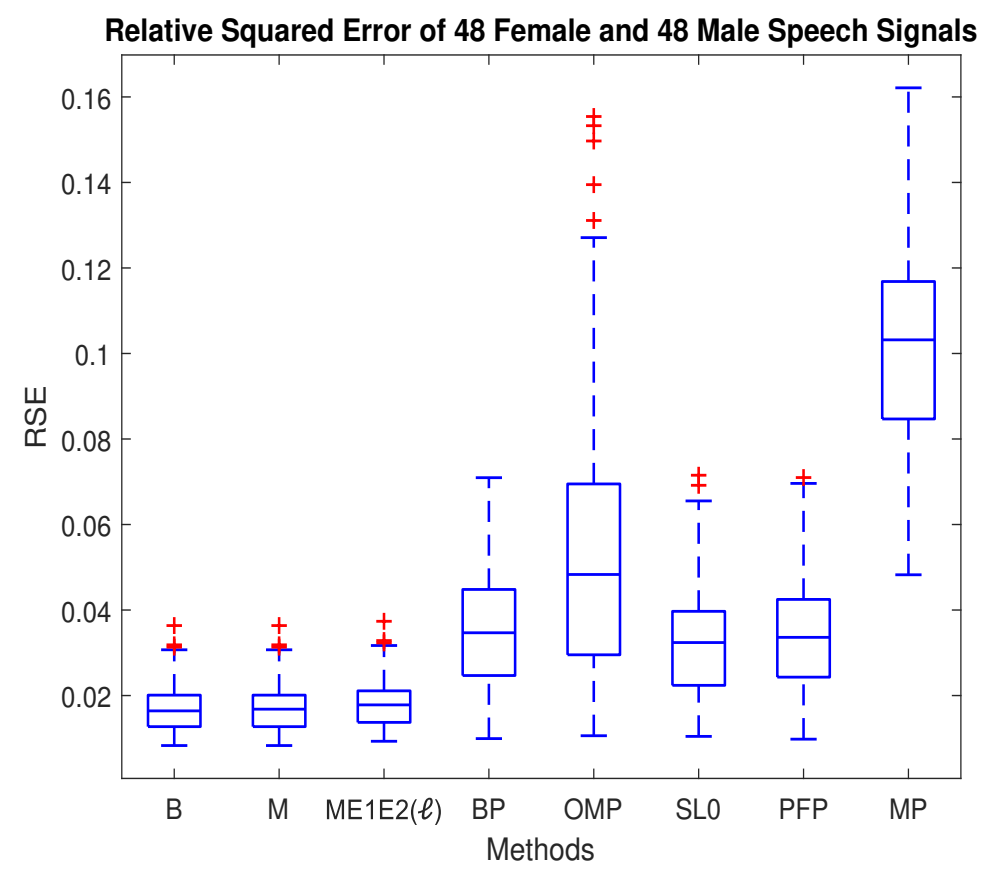

Figure 6.13: Average RSEs of 96 recovered signals.

Fig. 6.14 evaluates the quality of the recovered signals using the Perceptual Evaluation Speech Quality (PESQ). The average PESQ score for recovered female speech signals is better than that for recovered male speech signals. For both male and female speech signals, the MAX FS algorithms outperform the others, providing the highest PESQ score of 4.3 for female speech signals. OMP provides the highest average PESQ score among the traditional recovery algorithms, yet its highest PESQ score is slightly more than 2.5 , indicating poor quality. 

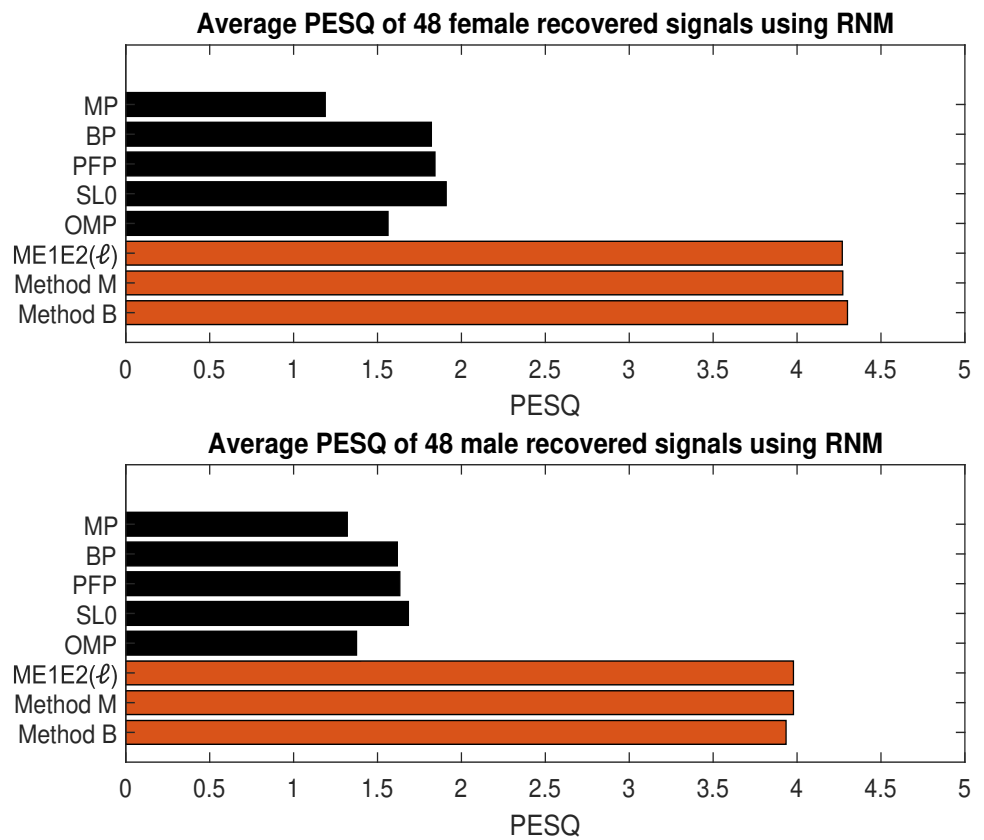

Figure 6.14: Comparing average PESQ for 48 female and 48 male recovered speech signals using RNM.

The spectrograms and the frequency responses of the linear predictor coefficients of the recovered and original speech signals of a randomly selected male and randomly selected female speech signal are presented in Figs. 6.15,6.16, The spectrograms of the female sample FGJD0_SX9 and the male sample $M A E O 0_{-} S A 2$, both the original signal and the recovered signal, are obtained using 16ms Hamming window. To improve the FFT performance, a length that is an exact power of two is chosen. The number of data points used for the FFT in each block is 1024 .

Table 6.20 Table 6.21 compare the recovered sparsity and the formants of the MAX FS methods and the existing heuristics with the sparsity and formants of the original female speech signal FGJD0_SX9 and male sample $M A E O 0_{-} S A 2$ for $\mathrm{CR}=50 \%$. The MAX FS methods recover the exact sparsity while following the formants of the original signal. Also, the first three formants of the recovered signals are visually similar to the first three formants of both female and male original signals. The 
existing algorithms show good performance in following the original signal formants but they fail in estimating sparsity.
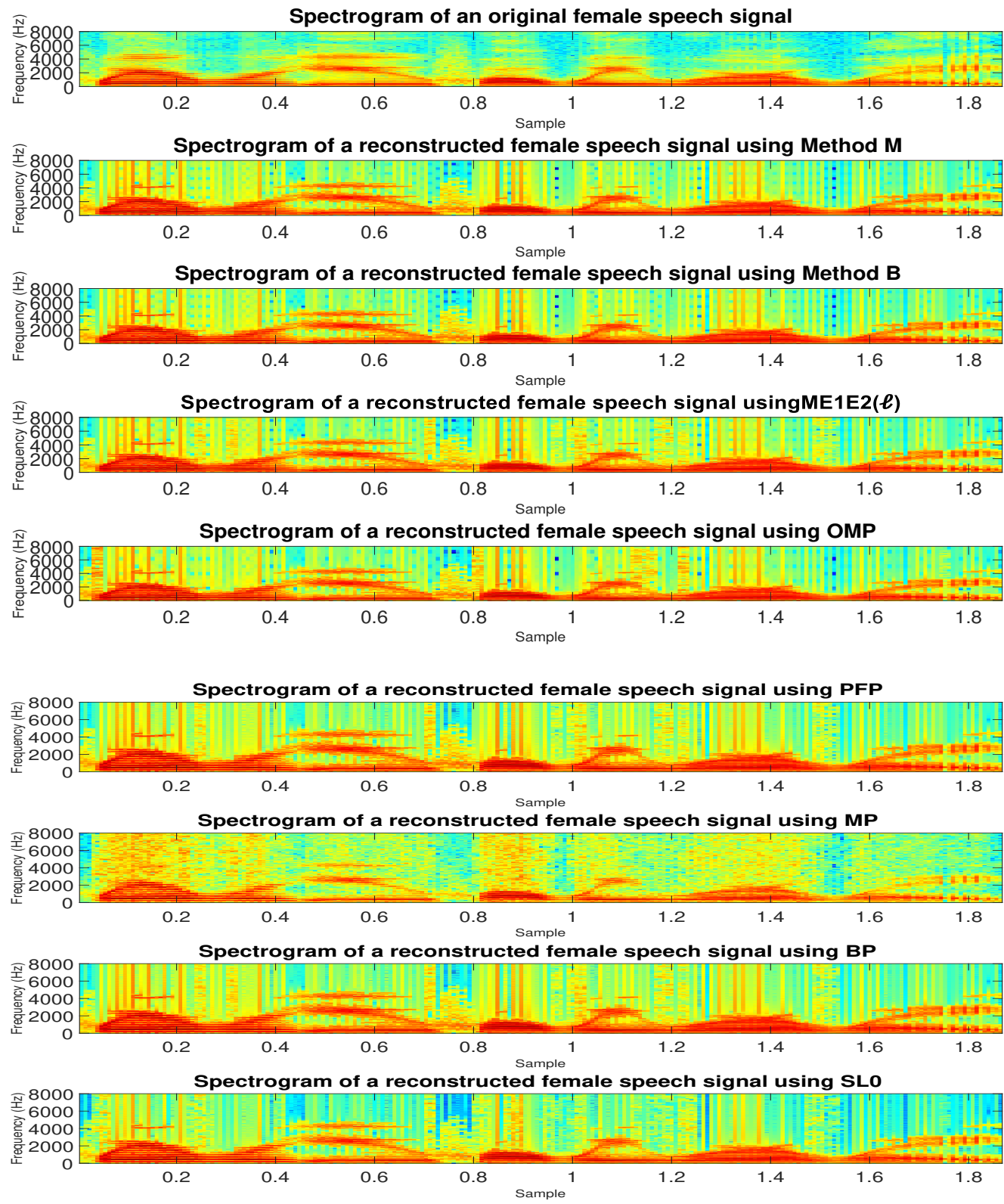

Figure 6.15: Comparing spectrogram of input female speech signal $F G J D 0_{S} X 9$ and reconstructed signals $(\mathrm{CR}=50 \%, \mathrm{RNM})$. 

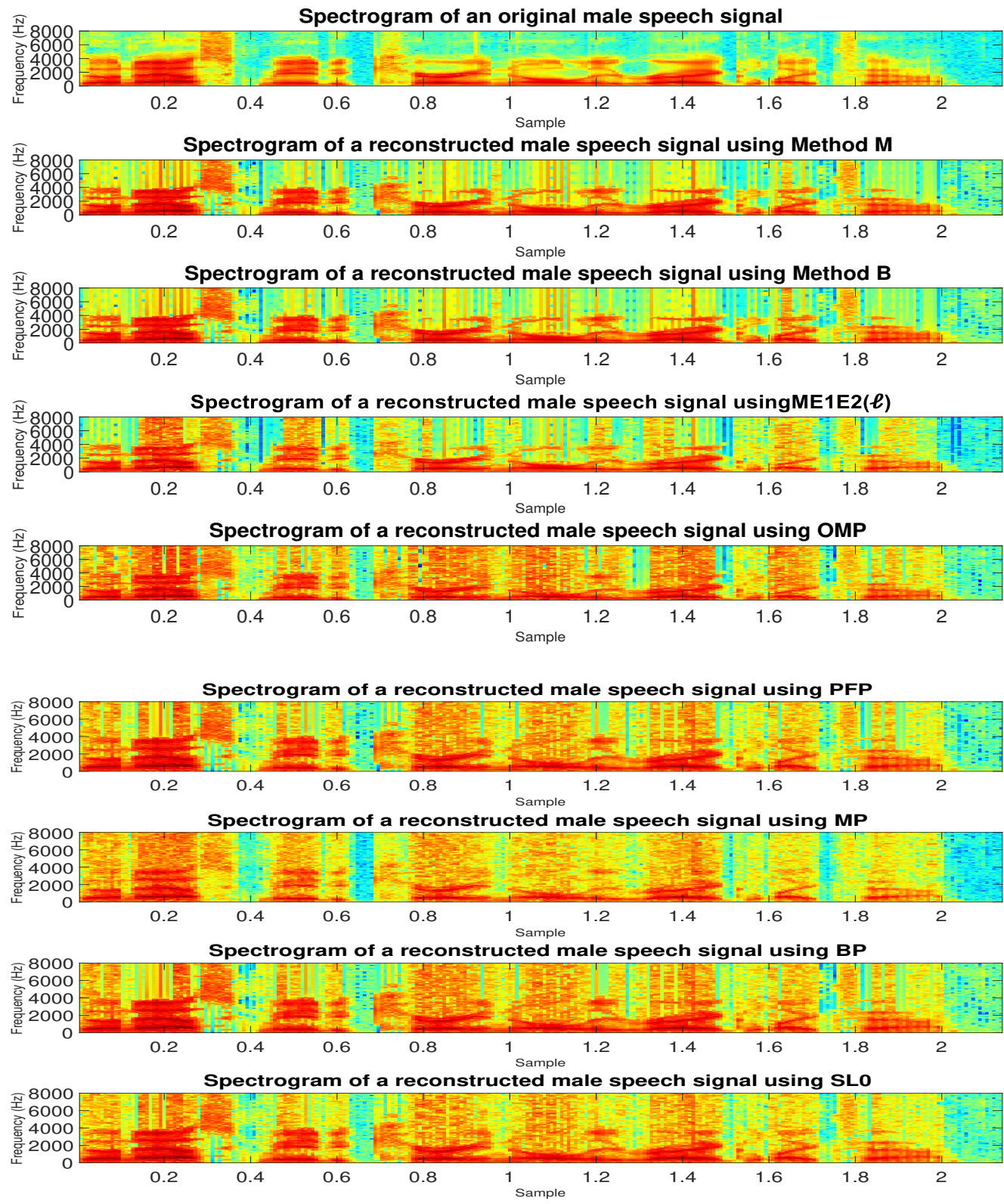

Figure 6.16: Comparing spectrogram of input male speech signal $M A E O 0_{S} A 2$ and reconstructed signals $(\mathrm{CR}=50 \%, \mathrm{RNM})$. 
Table 6.20: Comparing formants and sparsity level of the female original signal with the recovered signal using RNM.

\begin{tabular}{|c|c|c|c|c|c|c|}
\cline { 2 - 7 } \multicolumn{1}{c|}{} & Sparsity & F1 & F2 & F3 & F4 & F5 \\
\hline Original & 8529 & 559.0 & 1012.0 & 2028.4 & 2983.0 & 4218.3 \\
\hline MP & 40175 & 559.9 & 1004.9 & 2076.5 & 3087.2 & 4228.8 \\
\hline BP & 12169 & 560.1 & 1009.6 & 2039.0 & 3024.2 & 4225.6 \\
\hline SL0 & 38480 & 562.5 & 1015.7 & 2056.6 & 3039.8 & 4231.6 \\
\hline PFP & 11822 & 560.3 & 1010.7 & 2036.3 & 3023.4 & 4225.0 \\
\hline OMP & 12653 & 558.5 & 1010.2 & 2019.4 & 2989.3 & 4228.7 \\
\hline B & 8529 & 557.6 & 1011.5 & 2016.9 & 2988.8 & 4205.8 \\
\hline M & 8529 & 557.6 & 1011.5 & 2016.9 & 2988.8 & 4205.8 \\
\hline ME1E2( $\ell)$ & 8529 & 557.6 & 1011.6 & 2016.8 & 2988.6 & 4205.7 \\
\hline
\end{tabular}

Table 6.21: Comparing formants and sparsity level of the male original signal with the recovered signal using RNM.

\begin{tabular}{|c|c|c|c|c|c|c|}
\cline { 2 - 7 } \multicolumn{1}{c|}{} & Sparsity & F1 & F2 & F3 & F4 & F5 \\
\hline Original & 12579 & 449.4 & 1452.1 & 2327.1 & 3050.3 & 3894.3 \\
\hline IRWLS & 30083 & 463.1 & 1463.7 & 2464.6 & 3320.5 & 4209.5 \\
\hline MP & 50314 & 447.2 & 1458.0 & 2341.7 & 3179.9 & 3875.3 \\
\hline BP & 20548 & 448.0 & 1459.1 & 2338.4 & 3141.1 & 3887.8 \\
\hline SL0 & 49865 & 448.3 & 1452.9 & 2334.5 & 3134.8 & 3881.2 \\
\hline PFP & 19316 & 447.7 & 1459.4 & 2337.1 & 3126.0 & 3889.0 \\
\hline OMP & 26862 & 448.7 & 1451.5 & 2326.5 & 3030.0 & 3883.7 \\
\hline B & 13288 & 448.4 & 1453.0 & 2334.1 & 3080.4 & 3882.1 \\
\hline M & 13417 & 448.2 & 1451.9 & 2328.5 & 3048.7 & 3889.4 \\
\hline ME1E2($(\ell)$ & 13462 & 448.3 & 1451.1 & 2328.6 & 3046.1 & 3889.6 \\
\hline
\end{tabular}




\section{Chapter 7}

\section{New MAX FS Algorithm for}

\section{Non-Negative Matrix Factorization (NNMF)}

In an age of "big data", a fundamental concern in many fields such as signal processing, data mining, and machine learning is finding a useful form to represent data in fewer dimensions. This has the advantage of reducing the storage space requirements. More importantly, a smaller number of dimensions reveals information about the hidden structure in the data. For example, all of the original data can be reconstructed from a much smaller number of fundamental dimensions. In many cases, the raw or primitive data set is arranged as data matrices. Dimensionality reduction from the algebraic perspective can be regarded as decomposition of the original matrix into two lower rank matrices. This transformation to a lower-dimensional space improves the tractability of data, the required storage space and also when data is used with learning approaches, it enables better interpretation of parameters of the learnt model. 
The canonical methods for dimensionality reduction, namely Singular Value Decomposition (SVD [214], Principal Component Analysis (PCA] 215], Linear Discriminant Analysis (LDA) [216], Independent Component Analysis (ICA] 217], Vector Quantization (VQ) [218], differ from one another in their underlying structures and constraints. However, they are similar in that they do not place any constraints on the signs of components in the decomposed matrices. Therefore, the data is represented as a linear combination of positive and negative components of factorized matrices. The existence of the negative elements contradicts physical reality in many applications when the data matrix does not contain negative elements. For instance, a gray-scale face image which contains no negative intensities cannot be interpreted by negative components. To address this problem, several researchers suggested that non-negativity constraints should be incorporated in the dimensionality reduction process. This idea introduced a new paradigm, which is called Non-negative Matrix Factorization (NNMF).

In the 1990s, the effectiveness of NNMF, which was at first referred to as positive matrix factorization, was noticed by many researchers, especially Paatero and Tapper [219]. In 1999, it gained popularity through Lee and Seung's seminal paper published in Nature [220]. After this work, NNMF attracted a tremendous amount of research from signal separation to computer vision and machine learning.

This chapter focuses on developing and investigating new NNMF algorithms for solving the column subset selection problem. In Section 7.2 , the literature widely used for solving column subset selection problem is summarized. Then, Section 7.3 details three newly developed NNMF algorithms based on solutions for the MAX FS problem. Section 7.4 explains the experimental setup to investigate the performance of the new algorithms using synthetic datasets without outliers, synthetic datasets with outliers, and real-world image datasets. Section 7.5 presents empirical results, which show: (1) 
better performance of the newly developed algorithms compared to the state-of-theart NNMF methods in terms of finding low-dimensional data structure, (2) robustness of the developed algorithms to outliers compared to the widely used NNMF methods, and (3) higher solution accuracy of the newly introduced method for real-world image datasets compared to the state-of-the-art NNMF methods.

\subsection{Non-Negative Matrix Factorization (NNMF)}

Non-negative matrix factorization is an unsupervised dimension reduction method which determines a lower rank approximation of a given non-negative matrix $V \in$ $\mathbb{R}^{m \times n}$ as follows:

$$
\mathbf{V}_{m \times n} \approx \mathbf{W}_{m \times P} \mathbf{H}_{P \times n}
$$

where $P<<\min (m, n)$ is the rank or the size of the new subspace and non-negativity is imposed on all components of $\mathbf{W}$ and $\mathbf{H}$. NNMF estimates each non-negative input data vector in $\mathbf{V}$ by an additive linear combination of $P$ non-negative basis columns in $\mathbf{W}$, with non-negative coefficients in the corresponding column in $\mathbf{H}$. Therefore, $\mathbf{W}$ and $\mathbf{H}$ are regarded as the basis matrix and the coefficient matrix, respectively. Also, the product term $\mathbf{W H}$ is called the compressed version of the $\mathbf{V}$ or the approximating matrix of $\mathbf{V}$.

There are several NNMF problems:

NNMF1: Given a non-negative matrix $\mathbf{V} \in \mathbb{R}^{m \times n}$ and a positive integer $P<<$ $\min (m, n)$, find the optimal non-negative matrices $\mathbf{W} \in \mathbb{R}^{m \times P}$ and $\mathbf{H} \in \mathbb{R}^{P \times n}$ to minimize the difference between $\mathbf{V}$ and $\mathbf{W H}$.

NNMF2: Given a non-negative matrix $\mathbf{V} \in \mathbb{R}^{m \times n}$, find the optimal (where "optimal" means minimizing the selected error measure) number of factors or 
rank, $P$.

NNMF3: Given a non-negative matrix $\mathbf{V} \in \mathbb{R}^{m \times n}$ and a positive integer $P<<$ $\min (m, n)$, find the initial rank- $P$ nonnegative factors $\mathbf{W} \in \mathbb{R}^{m \times P}$ and $\mathbf{H} \in$ $\mathbb{R}^{P \times n}$.

NNMF4: Given a non-negative matrix $\mathbf{V} \in \mathbb{R}^{m \times n}$, select a subset of the columns of $\mathbf{V}$ (that is, $\mathbf{W}$ is a subset of the columns of $\mathbf{V}$ ), along with weighting factors $\mathbf{H}$, which can reconstruct the $\mathbf{V}$ with small error.

Finding an optimal solution for each of the mentioned NNMF problems serves an undeniable role in increasing or decreasing the accuracy in exchange for a smaller $P$ of the non-negative matrix factorization or dimension reduction process. NNMF1, is the generic problem, which is known to be NP-hard [221]. However, there exist many NNMF methods such as Multiplicative Update (MU) 222 to address the generic problem which can be selected based on the statistical characteristics of the input and the application in hand. Finding an optimal value of $P$ (NNMF2) is required by almost all the existing NNMF methods. This is called the rank selection problem. NNMF3, initialization problem, is an essential stage in NNMF, which can lead to better performance of the NNMF algorithms. NNMF4, the "column subset selection problem", can be considered as a subset of the other mentioned problems and is the core of our work. This work focuses on solving NNMF4. In the following sections, the state-of-the-art algorithms for the column subset selection problem (NNMF4) and their shortcomings are briefly explained. 


\subsection{Existing Algorithms for the NNMF Column Selection Problem}

NNMF4 addresses the rank selection problem and the initialization problem. In some cases, the matrix $\mathbf{W}$ is initialized using data points such that $\mathbf{W}=\mathbf{V}_{i j}$ where $i \in\{1, \ldots, m\}, j \in\{1, \ldots, P\})$, and $P$ is the rank 223.224]. Therefore, the basis matrix $\mathbf{W}$ is contained within the dataset, $\mathbf{V}$. Then, $\mathbf{H}$ is obtained by solving the following problem:

$$
\min f(\mathbf{H})=\left\|\mathbf{V}-\mathbf{V}_{i j} \mathbf{H}\right\|_{F}^{2} \quad \text { s.t. } \quad h_{i j} \geq 0,
$$

where the subscript $F$ is used to indicate Frobenius norm.

This approach is appealing if the input matrix is separable 225]. In general, matrix $\mathbf{V}$ is $P$-separable if there is an index set $\mathbb{J}$ of cardinality $P$ such that $\mathbf{V}=\mathbf{V}_{i j} \mathbf{H}$ and $h_{i j} \geq 0$. The separability condition in NNMF leads to reconstructing all columns of $\mathbf{V}$ by only considering the subset of columns $\mathbb{J}$. In other words, matrix $\mathbf{V}$ is separable if it can be written as $\mathbf{V}=\mathbf{W H}=\mathbf{W}\left[\mathbf{I}_{P} \mid \mathbf{H}^{\prime}\right] \Pi$, where $\mathbf{I}_{P}$ is the identity matrix of size $P, \Pi$ is a permutation matrix, and $\mathbf{H}^{\prime}$ in a matrix whose sum of the elements of each columns is at most one. The selected subset has a meaningful interpretation and preserves the latent structure of $\mathbf{V}$. This assumption makes sense in several applications such as feature extraction, document classification and blind hyperspectral unmixing [226]. However, the existing methods for the column subset selection problem show poor performance when the data contains outliers or it is noisy or dense according to 227.

A meaningful column subset can be obtained through different methods and is not unique. According to 228, the state of the art column subset selection methods can be listed as Successive Projection Algorithm (SPA), Successive Nonnegative 
Projection Algorithm [SNPA [229], XRAY [230], Fast Gradient Method for Nonnegative Sparse Regression (FGNSR) [231], Accelerated Hierarchical Alternating Least Squares A-HALS algorithm 232, Generalized Separable NMF with a Fast Gradient Method GS-FGM [228, and minimum-volume NMF (MIN-VOL NMF algorithm [233]. Among the mentioned methods, SNPA, XRAY, and FGNSR show the same result as SPA 228]. Here, SPA, A-HALS, GS-FGM and MIN-VOL NMF are summarized for more clarification.

- Successive Projection Algorithm (SPA): One of the most widely used algorithms for the column subset selection problem is SPA, introduced in 2001 [234]. This method has been discussed in other works, such as [235 237]. The SPA procedure is summarized in Fig. 7.1.

Figure 7.1 Successive Projection Algorithm (SPA)

INPUT:

Nearly separable matrix $\mathbf{V}$.

Number of columns, rank, $P$.

Strongly convex function $f$.

STEP 0: $\mathbf{R}($ residual $) \leftarrow \mathbf{V}, \mathbb{J} \leftarrow \emptyset$

STEP 1: $]=1$.

$$
\begin{array}{ll}
\text { while } & \mathbf{R} \neq 0 \text { and } \boldsymbol{I} \leq P \quad \text { do } \\
& \beth^{*}=\operatorname{argmax}_{\beth} f(\mathbf{R}(:, \beth)) . \\
& c_{j}=\mathbf{R}\left(:, \beth^{*}\right) /\left\|\mathbf{R}\left(:, \beth^{*}\right)\right\|_{2} . \\
& \mathbf{R} \leftarrow\left(I-c_{j} c_{j}^{T}\right) \mathbf{R}=\mathbf{R}-c_{j}\left(c_{j}^{T} \mathbf{R}\right) . \\
& \mathbb{J}=\mathbb{J} \cup \beth^{*}, \boldsymbol{J}=\boldsymbol{I}+1 .
\end{array}
$$

\section{end while}

OUTPUT: Index set $\mathbb{J}$ such that $\mathbf{V} \approx \mathbf{V}(:, \mathbb{J}) \mathbf{H}$ where $h_{i j} \geq 0$.

The index set $\mathbb{I}$ with cardinality of $P$ is defined such that $\mathbf{V} \approx \mathbf{V}_{i j} \mathbf{H}$ where $i \in\{1, \ldots, m\}, j \in\{1, \ldots, P\}), P$ is the rank, and $h_{i j} \geq 0$. SPA is a widely used separable NNNMF methods. However, it not robust to outliers. A more robust version of SPA is Robust Successive Projection RSPA Algorithm, which is very similar to SPA. Readers may refer to [238 to find more details about RSPA 
algorithm.

- Minimum-Volume NMF (MIN-VOL NMF) algorithm: The MinimumVolume NMF (MIN-VOL NMF) algorithm [239] uses the Projected Fast Gradient Method (PFGM [229] to update $\mathbf{H}$ and assumes that the sum of the components of each column of $\mathbf{H}$ is smaller or equal to one. This model ( Eqn. 7.3 consists of three key elements; the choice of: (1) the volume regularizer, (2) $\delta$, and (3) penalty parameter $\lambda$. The measure that is used to to minimize the volume of the columns of $\mathbf{W}$ is $\log \left(\operatorname{det}\left(\mathbf{W}^{T} \mathbf{W}+\delta \mathbf{I}\right)\right)$ where $\operatorname{det}\left(\mathbf{W}^{T} \mathbf{W}\right)=\prod_{j=1}^{P} \sigma_{i}^{2}(\mathbf{W}), \sigma_{i}^{2}$ are singular values of $\mathbf{W}$ and $\delta>0$ (the log term allows reducing large singular values) 233,240. Mathematically speaking, the basis matrix belongs to a $I$-dimensional subspace, $I<P$, such that $\mathbf{W}=\mathbf{U S}$ where $\mathbf{U} \in \mathbb{R}^{m \times \boldsymbol{I}}$ and $\mathbf{S} \in \mathbb{R}^{\mathbf{I} \times P}$ are an orthogonal basis and the coordinates of the columns of $\mathbf{W}$ in that subspace, respectively. Therefore, this regularization, $\log \left(\operatorname{det}\left(\mathbf{W}^{T} \mathbf{W}+\delta \mathbf{I}\right)\right)=\sum_{i=1}^{\mathbf{J}} \log \left(\sigma_{i}^{2}(\mathbf{S})+\delta\right)+(P-\mathbf{J}) \log (\delta)$, is meaningful even if the rank of matrix $\mathbf{W}$ is smaller than $P . \delta$ should not be too large or too small. It is recommended to choose $\delta$ between 0.1 and $10^{-3}$ [239]. Also, the choice of $\lambda$ is affected by the choice of $\delta$, the smaller $\delta$, the larger $|\log (\delta)|$. Therefore, $\lambda$ needs to be smaller in order to balance the two terms in the objective function. 239 recommended choosing $\tilde{\lambda}$ between 1 and $10^{-3}$. Fig. 7.2 indicates the steps of MIN-VOL NMF method.

$$
\min _{\mathbf{W} \geq 0, \mathbf{H}(:, j) \in \Delta^{P}{ }_{j}}\|\mathbf{V}-\mathbf{W H}\|_{F}^{2}+\lambda \log \left(\operatorname{det}\left(\mathbf{W}^{T} \mathbf{W}+\delta \mathbf{I}\right)\right)
$$

where $\Delta^{P}=\left\{x \in \mathbb{R}_{+}^{P} \mid \sum_{i} x_{i} \leq 1\right\}$.

- Accelerated Hierarchical Alternating Least Squares (A-HALS) : Hier- 
Figure 7.2 Minimum-Volume NMF (MIN-VOL NMF) algorithm

INPUT:

Nearly separable matrix V.

Number of columns, rank, $P$.

Number of iterations $t, \delta>0$, and $\tilde{\lambda}>0$.

STEP 0: Initialize $\mathbf{W}, \mathbf{H}$ using SNPA method [229].

STEP 1: let $\lambda=\tilde{\lambda} \frac{\|\mathbf{V}-\mathbf{W H}\|_{F}^{2}}{\log \left(\operatorname{det}\left(\mathbf{W}^{T} \mathbf{W}+\delta \mathbf{I}\right)\right)}$.

for $\mathbf{I}=1,2, \ldots, t$ do

Update $\mathbf{W}$ :

Let $\mathbf{A}=\mathbf{H H}^{T}+\lambda\left(\mathbf{W}^{T} \mathbf{W}+\delta \mathbf{I}\right)^{-1}$ and $\mathbf{C}=\mathbf{V} \mathbf{H}^{T}$.

Initialize $\mathbf{U}=\mathbf{W}$ and perform PFGM on problem:

$\min _{\mathbf{U} \geq 0} \frac{1}{2}\left\langle\mathbf{U}^{T} \mathbf{U}, \mathbf{A}\right\rangle-\langle\mathbf{U}, \mathbf{C}\rangle$

Set $\mathbf{W}$ as last iterate.

Update $\mathbf{H}$ :

perform PFGM on problem:

end for

$\min _{\mathbf{H}(:, j) \in \Delta^{P} \forall_{j}}\|\mathbf{V}-\mathbf{W H}\|_{F}^{2}$

OUTPUT: $\mathbf{W} \in \mathbb{R}^{m \times P}$ and $\mathbf{H} \in \mathbb{R}^{P \times n}$ such that $(\mathbf{W}, \mathbf{H})$ is an estimate of the matrix $\mathbf{V}$.

archical Alternating Least Squares, HALS, which solves the NNMF1 problem, updates a single column of $\mathbf{W}$ when all components of $\mathbf{W}$ are fixed. HALS is very efficient in practice. However, Gillis et al. 232] proposed a faster variant by limiting the maximum number of inner iterations of HALS algorithm to $[1+\alpha \rho w]$ where $\rho w=1+\frac{I+n P}{m P+m}$ and the optimal value of the parameter $\alpha \geq 0$ for A-HALS method is 0.5 based on 232. The procedure of A-HALS is outlined in Fig. 7.3 .

- Generalized Separable NMF with a Fast Gradient Method (GSFGM): In 2019, the GS-FGM method was proposed as a convex optimization model, which uses a fast gradient method [228. In Fig. 7.4, the steps of GS-FGM are presented (refer to 228] for details). 
Figure 7.3 Accelerate Hierarchical Alternating Least Squares (A-HALS)

INPUT:

Nearly separable matrix $\mathbf{V}$.

Initialize $\mathbf{W}^{(0)} \in \mathbb{R}^{m \times P}$ and $\mathbf{H}^{(0)} \in \mathbb{R}^{P \times n}$.

STEP 1:

for $\beth=0,1,2, \ldots$ do

Compute $\mathbf{A}=\mathbf{V} \mathbf{H}^{(\beth) T}$ and $\mathbf{B}=\mathbf{H}^{(\beth)} \mathbf{H}^{(\beth) T} ; \mathbf{W}^{(\beth, 0)}=\mathbf{W}^{(\beth)}$;

for $j=1:[1+\alpha \rho w]$ do

Obtain $\mathbf{C}_{: \beth}=\sum_{j=1}^{r-1} \mathbf{W}_{: j}^{\beth+1} \mathbf{B}_{j \beth}+\sum_{j=r+1}^{P} \mathbf{W}_{: j}^{(\beth)} \mathbf{B}_{j \beth}$;

$\mathbf{W}_{: \beth}=\max \left(0, \frac{\mathbf{A}_{: I}-\mathbf{C}_{: I}}{\mathbf{B}_{I J}}\right)$;

if $\left\|\mathbf{W}^{(\beth, j)}-\mathbf{W}^{(\beth, j-1)}\right\|_{F} \leq \epsilon\left\|\mathbf{W}^{(\beth, j)}-\mathbf{W}^{(\beth, 0)}\right\|_{F}$ then

break;

end if

end for

$\mathbf{W}^{(\beth+\mathbf{1})}=\mathbf{W}^{(\beth, j)}$

Compute $\mathbf{H}^{(\mathrm{I}+1)}$ from $\mathbf{H}^{(\mathrm{I})}$ and $\mathbf{W}^{(\mathrm{I}+1)}$. end for

OUTPUT: $\mathbf{W} \in \mathbb{R}^{m \times P}$ and $\mathbf{H} \in \mathbb{R}^{P \times n}$ such that $\mathbf{V} \approx \mathbf{W H}$.

Figure 7.4 Generalized Separable NMF with a Fast Gradient Method (GS-FGM)

INPUT:

Nearly separable matrix $\mathbf{V}$, number $r_{1}$ of columns and $r_{2}$ of rows to extract, and maximum number of iterations.

Initialize $\mathbf{W}^{(0)} \in \mathbb{R}^{m \times P}$ and $\mathbf{H}^{(0)} \in \mathbb{R}^{P \times n}, \alpha_{0} \leftarrow 0.05, L \leftarrow 2\left(\|(V)\|_{2}\right)^{2}$, and $\lambda$.

STEP 1:

for $t=1:$ maxitr do

$\mathbf{W}_{K} \leftarrow \mathbf{W}$ and $\mathbf{H}_{K} t \leftarrow \mathbf{H}$.

$\nabla_{\mathbf{W}} F(\mathbf{W}, \mathbf{H}) \leftarrow \mathbf{V}^{T} \mathbf{V W}+\mathbf{V}^{T} \mathbf{H V}-\mathbf{V}^{T} \mathbf{V}+\lambda \mathbf{I}_{n}$.

$\nabla_{\mathbf{H}} F(\mathbf{W}, \mathbf{H}) \leftarrow \mathbf{V} \mathbf{W} \mathbf{V}^{T}+\mathbf{H V V}^{T}-\mathbf{V} \mathbf{V}^{T}+\lambda \mathbf{I}_{m}$.

$\mathbf{W}_{n} \leftarrow K_{\Omega}\left(\mathbf{W}-\frac{1}{L} \nabla_{\mathbf{W}} F(\mathbf{W}, \mathbf{H})\right)$.

$\mathbf{H}_{n} \leftarrow K_{\Omega}\left(\mathbf{H}-\frac{1}{L} \nabla_{\mathbf{H}} F(\mathbf{W}, \mathbf{H})\right)$.

$\mathbf{W} \leftarrow \mathbf{W}_{n}+\beta_{t}\left(\mathbf{W}_{n}-\mathbf{W}_{K}\right)$.

$\mathbf{H} \leftarrow \mathbf{W}_{n}+\beta_{t}\left(\mathbf{W}_{n}-\mathbf{W}_{K}\right)$.

end for

STEP 2:

$\mathbf{I}_{1} \leftarrow$ post $-\operatorname{process}\left(\mathbf{W}, r_{1}\right)$

$\mathbf{I}_{2} \leftarrow$ post $-\operatorname{process}\left(\mathbf{H}, r_{2}\right)$

OUTPUT: $\mathbf{W} \in \mathbb{R}^{m \times P}$, and a set $P_{1}$ of column indices and a set $P_{1}$ of row indices such that $\mathbf{V} \approx \mathbf{W H}$. 
Here, the generalized separability assumption is defined as follows:

$$
\mathbf{V}=\mathbf{V}\left(:, \beth_{1}\right) K_{1}+K_{2} \mathbf{V}\left(\beth_{1},:\right)
$$

where $K_{1}\left(:, \boldsymbol{\beth}_{1}\right)=\mathbf{I}_{r 1}$ and $K_{2}\left(\beth_{2},:\right)=\mathbf{I}_{r 2} . \operatorname{In} F(\mathbf{W}, \mathbf{H})=\frac{1}{2}\|\mathbf{V}-\mathbf{V W}-\mathbf{H V}\|_{F}^{2}+$ $\lambda(\operatorname{trace}(\mathbf{W})+\operatorname{trace}(\mathbf{H})), \lambda$ is a penalty parameter, which balances the importance between the approximation error. In GS-FGM, $\lambda$ is initialized as follows:

$$
\lambda=\tilde{\lambda} \frac{\left\|\mathbf{V}-\mathbf{V} \mathbf{W}_{0}-\mathbf{H}_{0} \mathbf{V}\right\|}{2 r},
$$

where rank $P=r_{1}+r_{2}$ and $\tilde{\lambda} \in\left[10^{-3}, 10\right]$. 228 presented details about the initialization step and the details of GS-FGM method.

Some shortcomings of current methods using to solve NNMF problems are:

- Initialization adds one more step to the NNMF process. This is undesirable, but the speed of convergence and the approximation error of NNMF methods depends on the initialization stage. A random initialization does not provide a promising and unique result. Consequently, a wide variety of initialization methods have been proposed. Introducing a proper initialization method that leads to fast convergence and lower overall error is still an area that needs more research and improvement.

- As there has been no effective mechanism to determine the rank, $P$, of the basis matrices, most NNMF algorithms assume that $P$ is pre-specified and this can cause a problem in practical applications. Although there are some rank selection methods, finding the optimal rank in NNMF is still an unsolved question 241]. 
- According to [226], exact NNMF methods for small problems are still needed and this should be addressed.

- As mentioned in this chapter, the computer vision community has focused on the application of NNMF in pattern recognition, feature extraction, source separation, etc. Not much work has been carried out on the application of NNMF in data compression 242.

- According to previous studies, the NNMF algorithms are sensitive to outliers 243,244]. However, many real world datasets contain noise and outliers. If the NNMF method does not handle the outliers, the estimated matrix $\mathbf{W}$ can be significantly corrupted. Consequently, the low-dimensional data structure WH will not accurately recover $\mathbf{V}$. This is an incentive to propose a robust NNMF method that learns the basis matrix $\mathbf{W}$ of size $P$ while being robust to outliers.

\subsection{New NNMF Algorithms via MAX FS}

In [22], Chinneck mentioned the possibility of converting NNMF4 into the MAX FS problem. As explained in Section 7.1, the goal in NNMF is to reduce the number of columns in the matrix $\mathbf{W}$ or, equivalently, the number of rows of $\mathbf{H}$ as much as possible. NNMF is, therefore, a type of sparse recovery problem, and can be approached by a generalization of the MAX FS algorithms in which entire rows of $\mathbf{H}$ are removed in a single step, instead of a single variable as usual in MAX FS. In this section, the focus is on developing algorithms to solve the NNMF4 problem via MAX FS.

The NNMF4 dimensionality reduction problem is equivalent to initializing $\mathbf{W}$ as $\mathbf{V}$, that is, $\mathbf{W}=\mathbf{V}$ and then zeroing as many of the columns of $\mathbf{W}$ (or as many of rows 
of $\mathbf{H})$ as possible, while still satisfying all of the constraints, at least approximately. Initially, $\mathbf{W}$ and $\mathbf{H}$ are $m \times n$ and $n \times n$, respectively. Therefore, there are $m n$ constraints of the form $v_{i j}=w_{i 1} h_{1 j}+w_{i 2} h_{2 j}+\ldots+w_{i n} h_{n j}$ where $w_{i j}$ and $h_{i j}$ are constants and variables respectively. The steps of three newly develped NNMF algorithms via MAX FS are explained in Section 7.3.1, Section 7.3.2, and Section 7.3.3, respectively.

\subsubsection{Feasible Subset Matrix Factorization (FSMF)}

Some details of the developed MAX FS NNMF algorithm called "Feasible Subset Matrix Factorization (FSMF)" can be summarized as follows:

- Versions of this LP are solved repeatedly: $\min Z=\sum_{i j} h_{i j} \quad$ s.t. $\quad \mathbf{V}=\mathbf{W H}$, $w_{i j} \geq 0$, and $h_{i j} \geq 0$.

- $\mathbf{W}$ is initialized equal to $\mathbf{V}$.

- The initial objective function $Z$ is the sum of all of the elements of matrix $\mathbf{H}$. As the algorithm progresses, the objective weights of some elements of $\mathbf{H}$ are reduced to zero.

- Candidates are rows of $\mathbf{H}$. The figure of merit for each candidate row is its sum of elements. Rowsthat are not already in the CoverSet, and with a sum of elements larger than a defined threshold (i.e. $10^{-6}$ ) are listed in the CandidateSet. Then, the candidates are sorted in decreasing order. CandidateSet contains indices of rows of $\mathbf{H}$.

- To speed-up the algorithm, the first ListLength candidates in the list can be tested, rather than all of them.

- Each candidate row of $\mathbf{H}$ in the list is tested by temporarily zeroing its objective function coefficients. This means that the candidate row has no cost as far as 
the LP solution is concerned. The LP is re-solved; afterwards the objective function coefficients are reset to 1.0 .

- In each outer iteration, the candidate that most reduces $Z$ when temporarily removed from the objective function is permanently removed from the objective function by reducing the objective function coefficients of the Winner row to zero. Then, the Winner is added to the CoverSet.

- Upon termination, the obtained CoverSet contains $P$ indices that are used to find $\widetilde{\mathbf{W}}$ and $\widetilde{\mathbf{H}}$. $\widetilde{\mathbf{W}}$ is given by the columns of $\mathbf{W}$ indexed by CoverSet. $\widetilde{\mathbf{H}}$ is given by the values in the final LP solution for the elements in the rows of $\mathbf{H}$ indexed by CoverSet.

As shown in Section 7.5, FSMF outperforms state-of-the-art column selection NNMF algorithms. FSMF has time complexity $O\left(n^{2}+n\right) O\left(n^{2.5}\right)$, where $O\left(n^{2.5}\right)$ is the LP time complexity 245]. The mentioned time complexity for FSMF is the worst-case complexity, where every remaining column is a candidate at each iteration instead of all $n$ columns. However, the speed of the algorithm needs to be improved to solve large real-world problems. To increase the speed of the proposed method two faster version of the FSMF are introduced in this work.

\subsubsection{Fast Feasible Subset Matrix Factorization Algorithm (FFSMF)}

The proposed Fast Feasible Subset Matrix Factorization (FFSMF) algorithm is a faster version of FSMF algorithm, which only solves a single LP. This LP identifies the CandidateSet, sorts it in descending order, and then takes the top $P$ members

of the sorted CandidateSet as the nonzero rows of $\mathbf{H}$. FFSMF greatly reduces the 
Figure 7.5 FSMF algorithm

STEP $0:$ CoverSet $\leftarrow \emptyset, \mathbf{W}^{(0)} \leftarrow \mathbf{V}$

STEP 1: Solve LP.

CandidateSet $\leftarrow$ ListLength top candidates (rows of $\mathbf{H}$ not already in

CoverSet. The figure of merit for each candidate is the sum of its elements. The candidates are sorted in decreasing order.)

STEP 2 : Winner $Z \leftarrow \infty$.

for each candidate row $k$ of $\mathbf{H}$, in CandidateSet do

Temporarily set the objective function coefficients of $h_{k j} \forall j$ to 0 .

Solve the reduced LP.

if $Z \leq 10^{-6}$ then

Add index $k$ to CoverSet.

Exit.

end if

if $Z<$ Winner $Z$ then

Winner $\leftarrow k$.

Winner $Z \leftarrow Z$.

NextCandidateSet $\leftarrow$ ListLength top candidates (rows of $\mathbf{H}$ not already in CoverSet, excluding $k$. The figure of merit for each candidate is the sum of its elements. The candidates are sorted in decreasing order.)

end if

Revert the objective function coefficients of $h_{k j} \forall j$ to 1.0 . end for

STEP 3 : Add Winner to CoverSet.

Permanently fix the objective coefficients of $h_{\text {Winner }} \forall j$ to 0.0 .

CandidateSet $\leftarrow$ NextCandidateSet.

Go to STEP 2.

OUTPUT: CoverSet contains indices of the reduced set of columns of $\mathbf{W}$ and rows of $\mathbf{H}$ that should be kept. The values of the elements of $\mathbf{H}$ are taken from the final LP solution. 
complexity and running time vs. FSMF algorithm since its time complexity is $O\left(n^{2.5}\right)$, where $O\left(n^{2.5}\right)$ is the LP time complexity [245]. The procedure of FFSMF is detailed in Fig. 7.6 .

The rank $P$ is prespecified in FFSMF algorithm. The alternative is specifying a range of $P$ to find out the rank, which provides better solution quality. However, there is a trade-off between algorithm processing time and the recovery quality. By specifying a range for $P$, one LP is solved for each value of $P$. Then, the obtained values of $Z$ are compared. Finally, a $P$ corresponding to the smallest value of $Z$ is selected.

Figure 7.6 FFSMF algorithm

STEP 0 : Cover Set $\leftarrow \emptyset, \mathbf{W}^{(\mathbf{0})} \leftarrow \mathbf{V}, P \leftarrow \operatorname{rank}$ (or a specified range for $P$ )

STEP 1: Solve LP.

CoverSet $\leftarrow P$ candidates (rows of $\mathbf{H}$. The figure of merit for each candidate is the sum of its elements. The candidates are sorted in decreasing order.)

STEP 2 : Remove the elements of the rows of $\mathbf{H}$ indicated by the first $P$ entries in CoverSet from the objective function $Z$. Solve the reduced LP.

OUTPUT: CoverSet contains indices of the reduced set of columns of $\mathbf{W}$ and rows of $\mathbf{H}$ that should be kept. The values of the elements of $\mathbf{H}$ are taken from the reduced LP solution.

\subsubsection{Feasible Subset Matrix Factorization - Partitioned (FSMF-P)}

In this new algorithm, the main idea is partitioning the columns of the given initial W matrix, and then solving an LP for each partition. Time complexity of FSMF-P is $O(P) O\left(n^{2.5}\right)$, where $P$ is the rank and $O\left(n^{2.5}\right)$ is the LP time complexity [245]. Therefore, FSMF-P is faster than FSMF for large $\mathbf{V}$ because it solves a set of much smaller LPs instead of the large LP(s) needed to address the entire $\mathbf{V}$ matrix simultaneously. In order to partition the constraints, the most important columns of the 
constraints should be spread out into different partitions. Scaling is used for the face image dataset to give each facial image the same importance as suggested in [228]. In the experiments, the following steps are considered to meet the mentioned goal.

1. Scaling the columns of $\mathbf{V}$ so that their $\ell_{1}$ norm is equal to one.

2. Using random partitioning.

The procedure of the FSMF-P algorithm is explained in Fig. 7.7. In the output, the values of the elements of $\mathbf{H}$ can either be taken from the LP solution in STEP 2 or using a fast NNMF method. In the experiments, HALS is used to obtain values of $\mathbf{H}$ after estimating the CoverSet using FSMF-P algorithm.

Figure 7.7 FSMF-P algorithm

STEP 0 : CoverSet $\leftarrow \emptyset, \mathbf{W}^{(0)} \leftarrow \mathbf{V}, P \leftarrow \operatorname{rank}$ Scale columns of $\mathbf{W}^{(\mathbf{0})}$.

STEP 1: Segment columns of $\mathbf{W}^{(\mathbf{0})}$ into $P$ partitions.

for each partition do Solve LP.

CoverSet $\leftarrow$ CoverSet $\cup$ (row of $\mathbf{H}$ having largest sum of elements) end for

STEP 2 : Remove the elements of the rows of $\mathbf{H}$ indicated by the $P$ entries in CoverSet from the objective function $Z$. Solve LP.

OUTPUT: CoverSet contains indices of the columns of $\mathbf{W}$ and rows of $\mathbf{H}$ that should be kept. The values of the elements of $\mathbf{H}$ are taken from the LP solution in STEP 2 or using a fast method such as HALS.

The following are the problems present in FSMF although can produce more accurate results. It may be noted that such problems are not present in FSMF-P algorithm which can produce more approximate results:

- FSMF is very slow compared to the state-of-the-art column selection NNMF methods since it needs to solve one LP for each candidate in the list. 
- FSMF is impractical for real world data sets due to the large number of constraints: $(m \times n)$. For instance, consider a dataset with $m=2016$ and $n=380$. In this case, there are 766,080 constraints.

The application of the newly developed algorithms is investigated in the following sections.

\subsection{Experimental Setup}

Three sets of experiments are considered in this section. The performances of the three proposed NNMF algorithms are compared with the state-of-the-art NNMF methods for: (1) synthetic datasets, (2) synthetic datasets with outliers, and (3) face image datasets. For the synthetic datasets, FSMF and FFSMF algorithms are compared in terms of the root mean square residual to measure how well the methods find a low-dimensional data structure $\mathbf{W H}$ to approximately recover $\mathbf{V}$. The second set of experiments investigates the robustness of the FSMF and FFSMF algorithms to outliers compared to the state-of-the-art algorithms. Finally, the FSMF-P algorithm and the comparators are applied on different real-world column subset selection problems.

\section{NNMF for Synthetic datasets:}

- Synthetic datasets: Let $P=10, m=50$, and $n=200$. Each entry of $\mathbf{V}_{m \times n}$ is generated randomly. We generated 100 such matrices.

- Comparators: FSMF and FFSMF algorithms are compared with 6 stateof-the-art methods mentioned in Section 7.2: (1) MIN-VOL NMF, (2) AHALS, (3) HALS, (4)MU, (5) SPA, and (6) RSPA. To equalize the time required by the algorithms, about 100 trials are used for the comparators in our experiments. 
- Rank selection: For this set of experiments, the value of $P$ is known in advance. FSMF terminates when the length of the Coverset reaches $P$.

- Initialization: The proposed methods, SPA, and RSPA do not need to initialize the $\mathbf{W}^{(\mathbf{0})}$ and $\mathbf{H}^{(\mathbf{0})}$ matrices. In the proposed methods, $\mathbf{W}^{(\mathbf{0})}$ is initialized equal to $\mathbf{V}$. The initialization for the other comparators can be summarized as follows:

- In MIN-VOL NMF, $\mathbf{W}^{(\mathbf{0})}$ and $\mathbf{H}^{(\mathbf{0})}$ are initialized as suggested in 239 by using the successive nonnegative projection algorithm (SNPA). Matrix $\mathbf{W}^{(\mathbf{0})}$ is initialized equal to $\mathbf{V}(:, \mathbb{I})$ where $\mathbb{J}$ is obtained with SNPA to handle the rank-deficient separable NNMF problem [229]. The initial $\mathbf{H}^{(\mathbf{0})}$ is obtained using SNPA, which minimizes $\left\|\mathbf{V}-\mathbf{W}^{(\mathbf{0})} \mathbf{H}^{(\mathbf{0})}\right\|_{F}^{2}$.

- According to [246], there is no clear initialization method for MU, HALS, and A-HALS. Therefore, random initialization is used for the mentioned algorithms.

- Evaluation metric: The root mean square residual is used to evaluate the performance of the methods for synthetic datasets.

$$
\text { RMSErrors }=\frac{\|\mathbf{V}-\mathbf{W H}\|_{2}}{\sqrt{m n}},
$$

where $m$ and $n$ are number of rows and columns of $\mathbf{V}$, respectively.

\section{NNMF for synthetic datasets with outliers}

- Synthetic data sets with outliers: In 2019, Gillis showed the superior performance of $\operatorname{RSPA}(d, p, \beta)$, which is robust to outliers, compared to SPA 238. Here, the same experiment is run to investigate the ability of FSMF to handle outliers by taking into account the percentage 
of correctly identified columns of $\mathbf{W}$. Let $P=10, n=200, r=10$ (i.e. number of outliers), and the value of $m$ is varied from 10 to 50 (i.e. $m \in\{10,20,30,40,50\})$. Each matrix $\mathbf{V}, \mathbf{W}$, and $\mathbf{H}$ is constructed as follows:

- Entries of $\mathbf{W}_{m \times P}$ are generated randomly using uniform distribution in the interval $[0,1]$.

- Entries of $\mathbf{H}_{P \times(n-r-P)}$ are generated in the same way as $\mathbf{W}$. Then, each column of $\mathbf{H}_{P \times(n-r-P)}$ is normalized as in Eqn. (7.7) since the sum of entries of each column of matrix $\mathbf{H}_{P \times(n-r-P)}$ should be at most one in order to have a separable matrix $\mathbf{V}$ (see Section 7.2 to find more details about separable assumption).

$$
h_{j}=\frac{h_{j}}{\left\|h_{j}\right\|_{1}},
$$

$h_{j}$ represents the $j$ column of the matrix $\mathbf{H}$.

- Matrix $\mathbf{V}_{m \times n}$ with outliers is generated in two steps: (1) First, $\mathbf{V}_{m \times(n-r)}$ is generated as $\mathbf{V}_{m \times(n-r)}=\mathbf{W}_{m \times P}\left[\mathbf{I}_{P} \mid \mathbf{H}_{P \times(n-r-P)}\right]$, and (2) $r=10$ outlier columns, whose entries are generated randomly using a normal distribution of mean zero and variance one, are added to the end of the matrix $\mathbf{V}_{m \times(n-r)}$ to obtain $\mathbf{V}_{m \times n}$. For each value of $m, 100$ such matrices are generated.

This creates $\mathbf{V}_{m \times n}$, which we know in advance is separable and has an index set $\mathbb{J}$ of cardinality $P$ such that $\mathbf{V}(:, \mathbb{J}) \mathbf{H}$ and $h_{i j} \geq 0$, and $P$ columns that we may or may not be able to reconstruct accurately (note that $\mathbf{W}=$ $\mathbf{V}(:, \mathbb{J})$ as described in Section 7.3). The goal is reconstruct accurately as many as possible of those $P$ columns. 
- Comparators: The performance of FSMF and FFSMF are compared with SPA and RSPA algorithms since Gillis showed the effectiveness of RSPA in the presence of outliers [238]. Also, different choices of the RSPA parameters that he mentioned in his work are analysed and compared with the proposed method.

- Rank selection: For this set of experiments, except FSMF, the comparators need to know the value of $P$ in advance.

3. NNMF for image datasets: The experiments are carried out on three different image datesets.

Yale face dataset: This dataset comprises face images of 38 people, each of which has 64 frontal face images with various occlusions incurred by different light conditions. The images of size $192 \times 168$ are downsampled to $48 \times 42$. Then, 10 face images from each individual are randomly selected to obtain 380 images. Finally, the pixel-by-face matrix has dimension 2016 $\times 380$.

Olivetti Research Laboratory (ORL) face dataset: This dataset consists of a series of face images taken between April 1992 and April 1994 by Olivetti laboratory in Cambridge, UK. The dataset contains 40 objects of different ages, genders and races. From each individual, ORL has 10 different images of size $112 \times 92$, which are downsampled to obtain images of size $23 \times 19$. The pixel-by-face matrix has dimension $437 \times 400$.

\section{Centre for Biological and Computational Learning (CBCL) face}

dataset: $\mathrm{CBCL}$ is a public database for research usage provided by the MIT center for Biological and Computational Learning. This dataset consists of 2429 face images of size $19 \times 19$ so that the input pixel-by-face 
matrix has dimension $361 \times 2429$.

The datasets are available at URL: https://github.com/b-fakhar/ Image-datasets.git. In the experiment, to give each facial image the same importance, they are scaled so that their $\ell_{1}$ norm is equal to one. Based on [228], the image size in the Yale and ORL datasets is too large for the purpose of this work since GS-FGM runs in $\mathrm{O}\left(m n^{2}+n m^{2}\right)$ operations. Therefore, it is impractical to apply GS-FGM on such data. After applying the algorithms on the downsampled datasets, columns selected by each algorithm identify the corresponding columns and rows in the original matrix (the datasets before downsampling).

- Comparators: In [228], the authors compared the GS-FGM algorithms with different state-of-the-art NNMF methods using the mentioned image datasets. Based on [228], GS-FGM outperforms other NNMF methods. Therefore, the performance of FSMF-P algorithm is compared to GS-FGM algorithm for this set of experiments. The execution time allowed for GSFGM is limited to the amount of time take by FSMF-P algorithm.

- Rank selection: The rank $P$ is chosen based on [228]. The values of $P$ for Yale, ORL, and CBCL datasets are 38, 40, and 49, respectively.

- Evaluation metric: The Relative Approximation Quality is used to evaluate the performance of the methods for image datasets as in [228].

$$
\text { Relative Approximation Quality }=\left(1-\frac{\|\mathbf{V}-\mathbf{W H}\|_{F}}{\|V\|_{F}}\right) \times 100
$$

where \|\|$_{F}$ indicates Frobenius norm.

- Software: All algorithms are implemented in Matlab version 2020, running 
in a Windows 10 environment. The linear programming solver is MOSEK via the MOSEK Optimization Toolbox for Matlab version 8.1.0.56 [47]. The code for comparison algorithms is available from https://sites.google.com/site/ nicolasgillis/code.

- Hardware: The information about hardware is provided in Section 6.4.1.6.

\subsection{Results and Discussion}

1. Synthetic datasets: The NNMF methods are compared in terms of the root mean square residual in Fig. 7.8 and Fig. 7.9 for better visualization. $\operatorname{RSPA}(d=$ $80, p=1, \beta=4$ ) (based on [238], RSPA has the best performance with these settings) and SPA with average root mean square residual of 2.54 show the poorest performance. MU has an average root mean square residual of 0.1356 and is the worst NNMF method after RSPA and SPA. FSMF provides the best performance with average root mean square residual of almost zero. FFSMF is the second best algorithm with an average root mean square residual of 0.0019 . However, FFSMF is faster than the FSMF by $99.62 \%$ on average. Therefore, there is a trade-off in error against speed.

2. Synthetic datasets with outliers: Fig. 7.10 reports the percentage of correctly identified columns of $\mathbf{W}$ by FSMF, FFSMF, SPA and RSPA with various combinations of the parameters. The experiment shows that except for the proposed algorithms, no algorithm is able to recover columns of $\mathbf{W}$ with high probability since the outliers and the columns of $\mathbf{W}$ are less separated and linearly dependant. However, the proposed method is able to recover $100 \%$ of the columns of $\mathbf{W}$ even when $m$ is small. As the norm of the outliers is larger than 


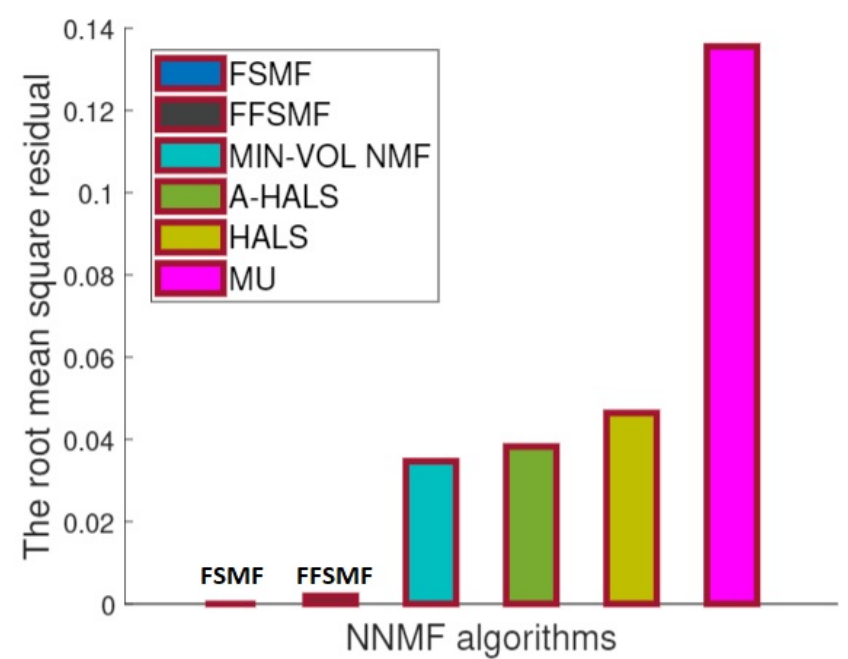

Figure 7.8: Comparing the average root mean square residual of the proposed algorithms with MIN-VOL NMF, A-HALS, HALS, and MU over 100 trials $(m=50, n=200$ and $P=10)$.

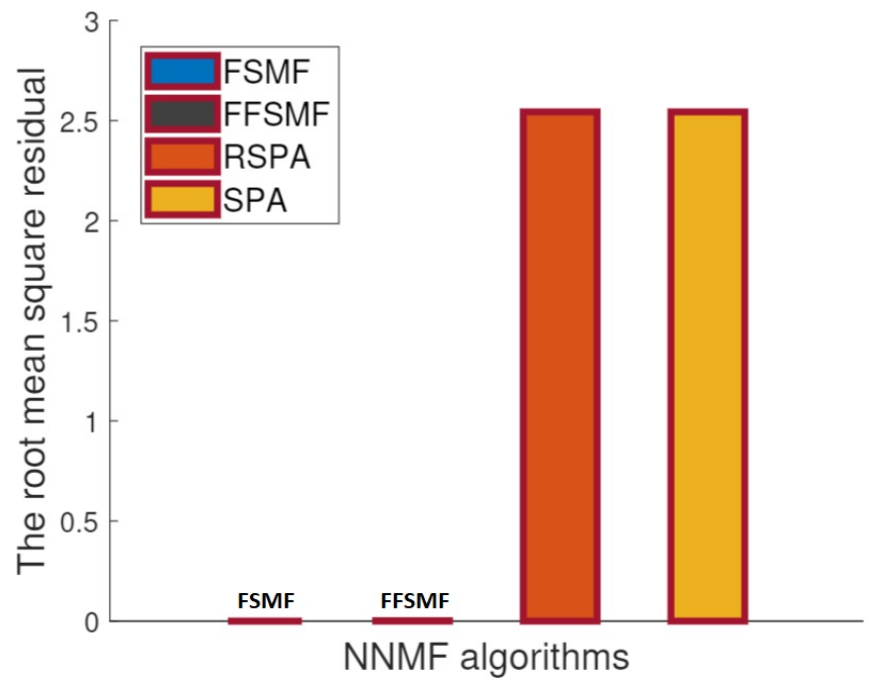

Figure 7.9: Comparing the average root mean square residual of the proposed algorithms with RSPA and SPA over 100 trials $(m=50, n=200$ and $P=10)$.

that of the columns of $\mathbf{W}$, SPA does not perform well. On the other hand, RSPA performs well under specific conditions (i.e. $d \geq 40, P=1$, and $\beta=4$ ). In summary, the proposed algorithms provide better performance for all values of $m$ and are robust to outliers. Moreover, FFSMF is faster than FSMF since 
it only needs to solve one LP to find the solution.

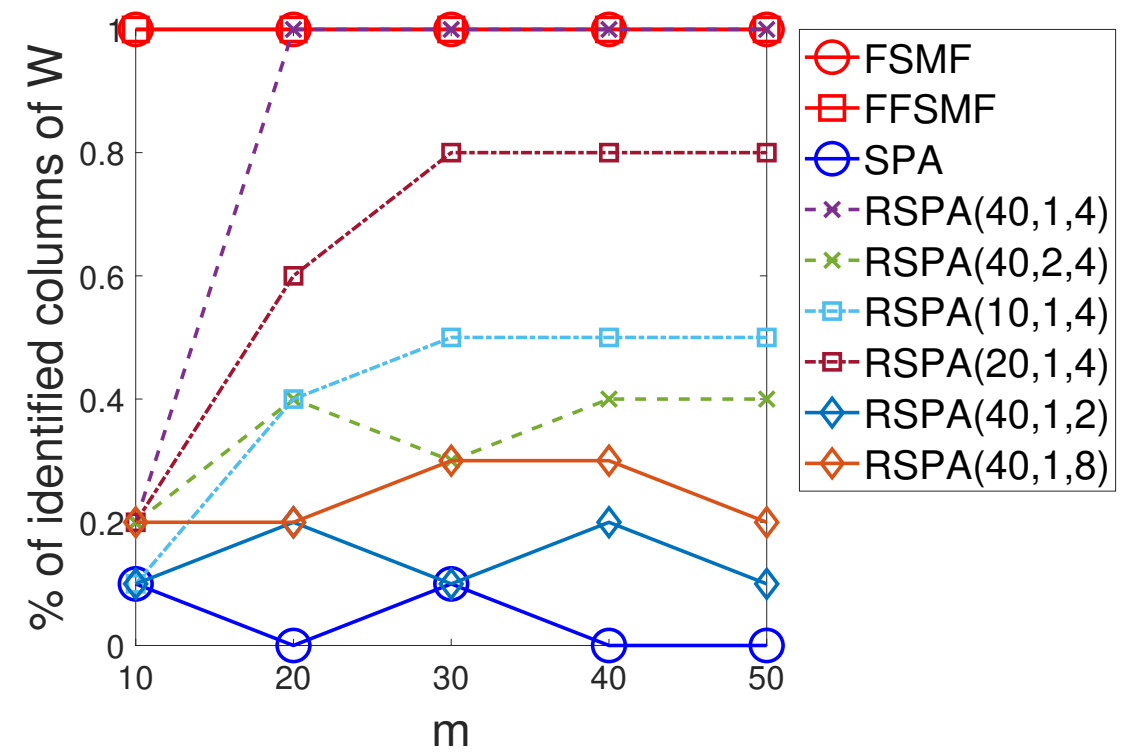

Figure 7.10: Percentage of recovered columns of $\mathbf{W}$ for the synthetic datasets using SPA, RSPA and NNMF MAX FS-based method.

3. Image datasets: The relative approximation quality of the factorizations provided by the FSMF-P algorithm and GS-FGM and their processing times in seconds are reported in Table 7.1 (the number of iterations of GS-FGM is 1000 as suggested in $[228]$ ). The highest quality is highlighted in bold. The proposed method outperforms GS-FGM for all datasets. The following figures provide visual representations of the solutions generated by FSMF-P algorithm and GS-FGM for each dataset. The faces selected for testing are for people who look rather different. 


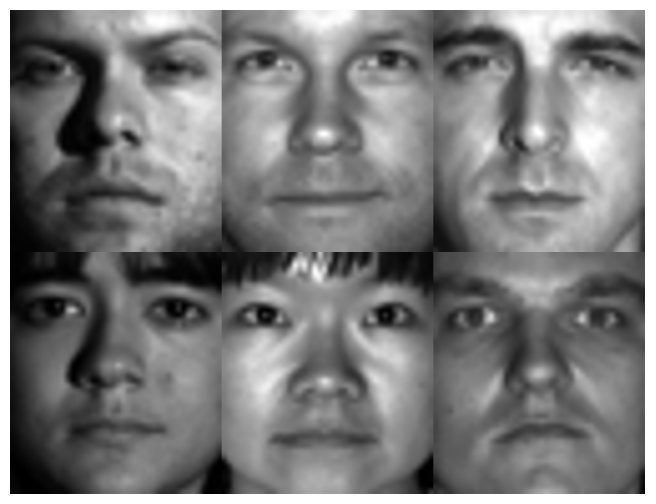

(a)

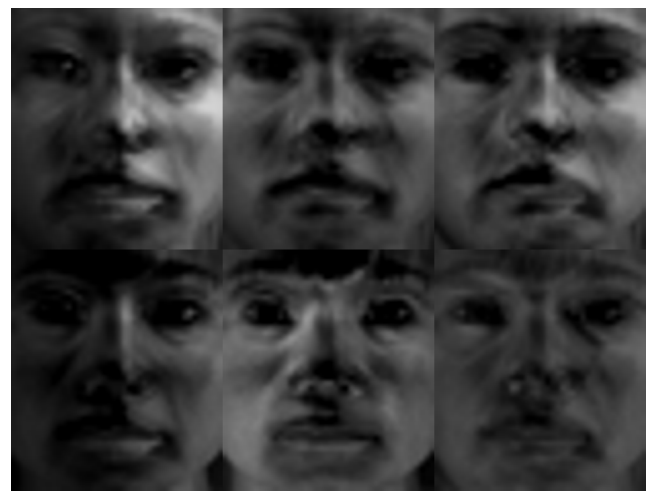

(b)

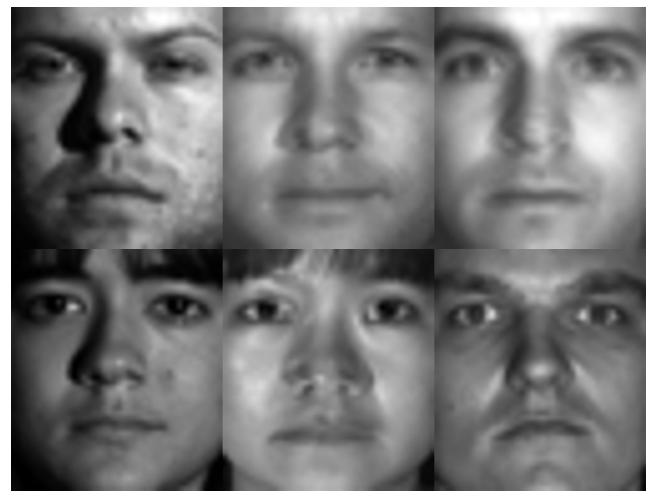

(c)

Figure 7.11: (a) 6 images from Yale dataset, (b) approximation by GS-FGM, (c) approximation by FSMF-P algorithm. 


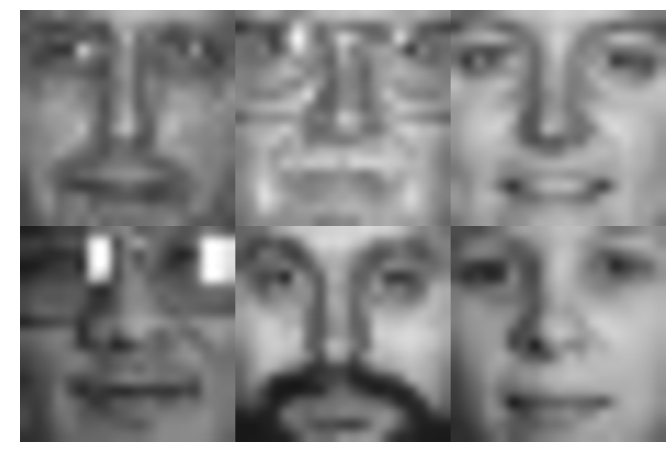

(a)

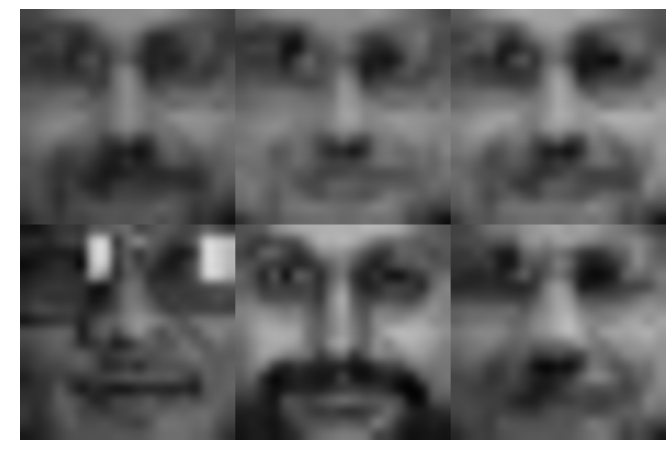

(b)

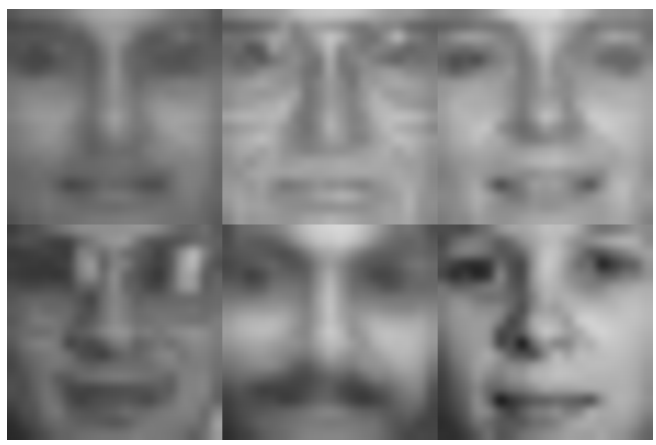

(c)

Figure 7.12: (a) 6 images from CBCL dataset, (b) approximation by GS-FGM, (c) approximation by FSMF-P algorithm. 


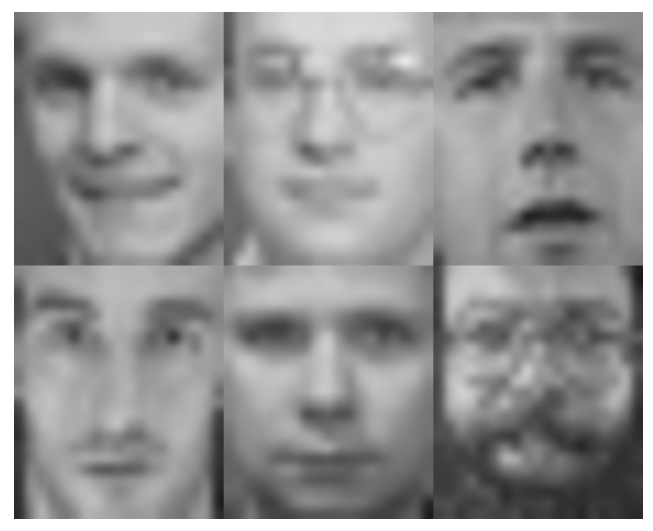

(a)

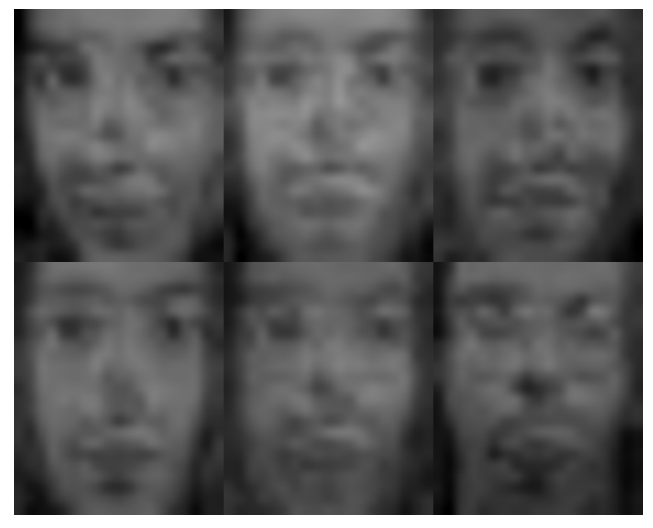

(b)

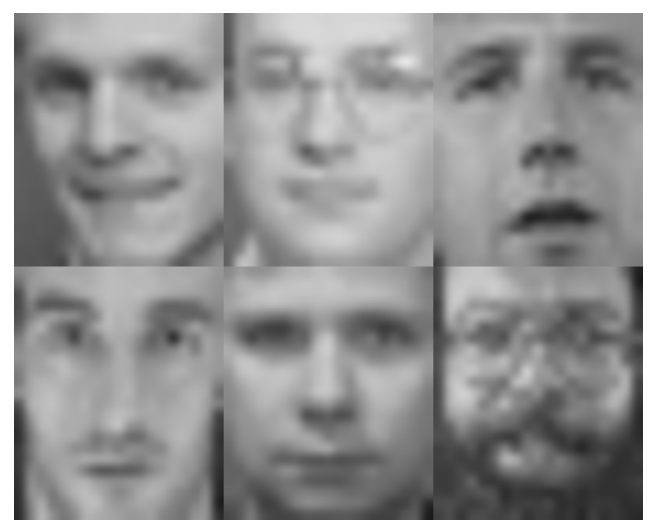

(c)

Figure 7.13: (a) 6 images from ORL dataset, (b) approximation by GS-FGM, (c) approximation by FSMF-P algorithm. 
Table 7.1: The relative approximation quality in percent for the facial image data sets.

\begin{tabular}{|l|c|c|c|}
\hline Dataset & P & GS-FGM & FSMF-P \\
\hline CBCL & 49 & 83.10 & $\mathbf{8 7 . 8 0}$ \\
\hline Yale & 38 & 68.26 & $\mathbf{7 4 . 5 5}$ \\
\hline ORL & 40 & 82.54 & $\mathbf{8 7 . 7 4}$ \\
\hline
\end{tabular}




\section{Chapter 8}

\section{Conclusions and Future Work}

This chapter summarizes the contributions presented in this thesis. Also, some open research issues for future research projects are discussed.

\subsection{Conclusions}

The problems stated in Chapter 3 have been solved in this thesis:

- The introduced strategies increase the speed of original MAX FS solution algorithms while preserving/improving solution quality for dense constraint matrices such as those found in the following applications:

- Binary classification. For binary classification, the best version of MAX FS is Algorithm 2E1. This improves the speed of the algorithm dramatically when compared to the original MAX FS algorithms, and also outperforms existing state-of-the-art algorithms on recall, while remaining competitive on other measures.

- Sparse recovery. For sparse recovery, the best version of MAX FS is Algorithm BE1E2 $(\ell)$. This new version of the algorithm is much faster 
than the original MAX FS method (Method B) while providing the same level of critical sparsity on synthetic datasets.

- High quality recovery of highly compressed signals. The proposed MAX FS CS sparse recovery algorithm is effective for recovery of compressively sensed ECG and speech signals. The best MAX FS algorithm for this application is $\operatorname{ME1E2}(\ell)$, which does not require prior knowledge of the input sparsity level. The critical sparsity of the proposed Algorithm ME1E2 $(\ell)$ is higher than that of the well-known CS sparse recovery algorithms. Therefore, it requires fewer measurements to recover compressed signals accurately. Also, this algorithm provides higher fidelity recovered signals because its solutions are sparser than those of CS conventional sparse recovery methods.

- Three proposed novel NNMF algorithms do not require initialization and among them the FSMF algorithm does not need pre-specified rank. According to the experimental results, the new algorithms outperform the state-of-the-art in the following applications:

- Dimensionality reduction. The new FSMF and FFSMF algorithms provide high quality dimensionality reduction compared to the state-of-theart NNMF methods. FSMF is faster than FFSMF, with a small reduction in the quality of the recovered matrix.

- Dimensionality reduction in the presence of outliers. The developed FSMF and FFSMF algorithms are robust to outliers in contrast to the state-of-the-art NNMF methods.

- Column subset selection problems. The introduced FSMF-P algorithm is a superior method for real-world column subset selection problems 
compared to the state-of-the-art GS-FGM algorithm.

\subsection{Summary of Contributions}

he following are the contributions of this dissertation in a nut shell:

1. Novel MAX FS algorithms for dense constraint matrices that are significantly faster than existing MAX FS algorithms, with little or no loss in solution accuracy.

1.1 Application of these algorithms in binary classification gives improved results in recall-oriented tasks such as disease diagnosis compared to existing widely used methods.

1.2 Much faster than existing MAX FS methods when applied in sparse recovery, with no loss in critical sparsity.

2. Fast MAX FS algorithm and example pipelines for CS sparse recovery that permit higher quality signal recovery from more highly compressed signals.

2.2 For recovery of highly compressed ECG signals, the developed algorithms along with the introduced pipeline provide higher performance compared to the well-known CS sparse recovery methods.

2.3 For recovery of highly compressed speech signals, the developed algorithms along with the introduced pipeline provide higher performance compared to the well-known CS sparse recovery methods.

3. Novel MAX FS-based NNMF algorithms, that do not require initialization in contrast to most of the existing NNMF methods. 
2.1 For dimensionality reduction, using the developed algorithms leads to better compression at higher quality than the state-of-the-art.

2.2 The introduced MAX FS-based NNMF algorithms are more robust to outliers compared to the state-of-the-art.

2.3 Application of these algorithms in column subset selection problem provides better solution quality compared to the state-of-the-art using realworld image datasets.

\subsection{Future Research}

There are many avenues for future research in (i) refinement and improvement of the methods developed in the dissertation, and (ii) new applications for the methods. Examples are given below.

1. Generalize the new MAX FS algorithms to other applications such as dictionary learning.

2. Extend the MAX FS algorithms by using an absolute error tolerance on the right hand side constant to handle the sparse recovery of noisy speech signals that have been compressively sensed. Also, investigate strategies to adaptively estimate absolute error tolerance parameter $\delta$.

3. Develop fast MAX FS extensions for less dense constraint matrices, including those found in general LPs.

4. Optimize the signal acquisition phase of the introduced CS sparse recovery pipelines. 
5. Extend the new MAX FS methods for muti-class classification and design a MAX FS-based decision tree construction algorithm.

6. Investigate the new MAX FS methods for training of neural networks.

7. Introduce and investigate different partitioning strategies for the new Feasible Subset Matrix Factorization - Partitioned (FSMF-P) algorithm to improve the solution quality.

8. Extend the new MAX FS methods for sparse recovery of 2-D signals. 


\section{References}

[1] S. Shilo, H. Rossman, and E. Segal, "Axes of a revolution: challenges and promises of big data in healthcare," Nature Medicine, vol. 26, no. 1, pp. 29-38, 2020.

[2] A. K. Tripathi, K. Sharma, M. Bala, A. Kumar, V. G. Menon, and A. K. Bashir, "A parallel military-dog-based algorithm for clustering big data in cognitive industrial internet of things," IEEE Transactions on Industrial Informatics, vol. 17, no. 3, pp. 2134-2142, 2021.

[3] G. Coluccia, C. Lastri, D. Guzzi, E. Magli, V. Nardino, L. Palombi, I. Pippi, V. Raimondi, C. Ravazzi, F. Garoi, D. Coltuc, R. Vitulli, and A. Z. Marchi, "Optical compressive imaging technologies for space big data," IEEE Transactions on Big Data, vol. 6, no. 3, pp. 430-442, 2020.

[4] Z. Zhou, X. Zhang, X. Zhou, and Y. Liu, "Semantic-aware visual abstraction of large-scale social media data with geo-tags," IEEE Access, vol. 7, pp. 114851114861, 2019.

[5] M. Chowdhury, A. Apon, and K. Dey, Data analytics for intelligent transportation systems. Elsevier, 2017. 
[6] A. Gersho and R. M. Gray, Vector quantization and signal compression, vol. 159. Springer Science \& Business Media, 2012.

[7] A. Mishra, F. Thakkar, C. Modi, and R. Kher, "ECG signal compression using compressive sensing and wavelet transform," in 2012 Annual International Conference of the IEEE Engineering in Medicine and Biology Society, pp. 34043407.

[8] T. S. Gunawan, O. O. Khalifa, A. A. Shafie, and E. Ambikairajah, "Speech compression using compressive sensing on a multicore system," in 2011 4th International Conference on Mechatronics (ICOM), pp. 1-4.

[9] Z. Yuan and E. Oja, "Projective nonnegative matrix factorization for image compression and feature extraction," in 2005 Scandinavian Conference on Image Analysis, pp. 333-342.

[10] M. W. Berry, M. Browne, A. N. Langville, V. P. Pauca, and R. J. Plemmons, "Algorithms and applications for approximate nonnegative matrix factorization," Computational Statistics \& Data Analysis, vol. 52, no. 1, pp. 155-173, 2007.

[11] J. W. Chinneck, "Fast heuristics for the maximum feasible subsystem problem," INFORMS Journal on Computing, vol. 13, no. 3, pp. 210-223, 2001.

[12] S. Jokar and M. E. Pfetsch, "Exact and approximate sparse solutions of underdetermined linear equations," SIAM Journal on Scientific Computing, vol. 31, no. 1, pp. 23-44, 2008.

[13] E. Amaldi, M. E. Pfetsch, and L. E. Trotter, "Some structural and algorithmic properties of the maximum feasible subsystem problem," in 1999 International 
Conference on Integer Programming and Combinatorial Optimization, pp. 4559.

[14] E. Amaldi, From finding maximum feasible subsystems of linear systems to feedforward neural network design. PhD dissertation, Swiss Federal Institute of Technology at Lausanne (EPFL), 1994.

[15] N. Chakravarti, "Some results concerning post-infeasibility analysis," European Journal of Operational Research, vol. 73, no. 1, pp. 139-143, 1994.

[16] E. Amaldi and V. Kann, "The complexity and approximability of finding maximum feasible subsystems of linear relations," Theoretical Computer Science, vol. 147, no. 1-2, pp. 181-210, 1995.

[17] J. K. Sankaran, "A note on resolving infeasibility in linear programs by constraint relaxation," Operations Research Letters, vol. 13, no. 1, pp. 19-20, 1993.

[18] K. P. Bennett and E. J. Bredensteiner, "A parametric optimization method for machine learning," INFORMS Journal on Computing, vol. 9, no. 3, pp. 311-318, 1997.

[19] O. L. Mangasarian, "Misclassification minimization," Journal of Global Optimization, vol. 5, no. 4, pp. 309-323, 1994.

[20] F. Rossi, S. Smriglio, and A. Sassano, "Models and algorithms for terrestrial digital broadcasting," Annals of Operations Research, vol. 107, no. 1, pp. 267283, 2001.

[21] M. Wagner, J. Meller, and R. Elber, "Large-scale linear programming techniques for the design of protein folding potentials," Mathematical Programming, vol. 101, no. 2, pp. 301-318, 2004. 
[22] J. W. Chinneck, "The maximum feasible subset problem (maxfs) and applications," INFOR: Information Systems and Operational Research, vol. 57, no. 4, pp. 496-516, 2019.

[23] E. Amaldi, "The maximum feasible subsystem problem and some applications," Agnetis A, Di Pillo G, editors. Modelli e Algoritmi perl'ottimizzazione di sistemi complessi. Pitagora Editrice Bologna, Italy, pp. 31-69, 2003.

[24] O. L. Mangasarian, "Misclassification minimization," Journal of Global Optimization, vol. 5, no. 4, pp. 309-323, 1994.

[25] O. Mangasarian, "Machine learning via polyhedral concave minimization," in Applied Mathematics and Parallel Computing, pp. 175-188, Springer, 1996.

[26] S. Dirkse, M. Ferris, and A. Meeraus, "Mathematical programs with equilibrium constraints: Automatic reformulation and solution via constraint optimization," in Technical Report NA-02/11, Oxford University Computing Laboratory, 2002.

[27] K. P. Bennett and E. J. Bredensteiner, "A parametric optimization method for machine learning," INFORMS Journal on Computing, vol. 9, no. 3, pp. 311-318, 1997.

[28] M. R. Parker, A set covering approach to infeasibility analysis of linear programming problems and related issues. $\mathrm{PhD}$ thesis, University of Colorado at Denver Denver, Colorado, 1995.

[29] N. Chakravarti, "Some results concerning post-infeasibility analysis," European Journal of Operational Research, vol. 73, no. 1, pp. 139-143, 1994. 
[30] M. Parker and J. Ryan, "Finding the minimum weight iis cover of an infeasible system of linear inequalities," Annals of Mathematics and Artificial Intelligence, vol. 17, no. 1, pp. 107-126, 1996.

[31] M. E. Pfetsch, The maximum feasible subsystem problem and vertex-facet incidences of polyhedra. PhD thesis, TU Berlin, Berlin, 2003.

[32] M. E. Pfetsch, "Branch-and-cut for the maximum feasible subsystem problem," SIAM Journal on Optimization, vol. 19, no. 1, pp. 21-38, 2008.

[33] W. Winston and M. Venkataramanan, "Operations research: Volume one; introduction to mathematical programming," Brooks/Cole, Cengage Learning, 2003.

[34] J. W. Chinneck, "An effective polynomial-time heuristic for the minimumcardinality iis set-covering problem," Annals of Mathematics and Artificial Intelligence, vol. 17, no. 1, pp. 127-144, 1996.

[35] J. W. Chinneck, "Sparse solutions of linear systems via maximum feasible subsets," SIAM Journal on Scientific Computing, pp. 1-23, 2018.

[36] E. Amaldi, M. Bruglieri, and G. Casale, "A two-phase relaxation-based heuristic for the maximum feasible subsystem problem," Computers $\&$ Operations Research, vol. 35, no. 5, pp. 1465-1482, 2008.

[37] E. Amaldi, P. Belotti, and R. Hauser, "Randomized relaxation methods for the maximum feasible subsystem problem," in 2005 International Conference on Integer Programming and Combinatorial Optimization, pp. 249-264.

[38] P. M. Pardalos, D.-Z. Du, and R. L. Graham, Handbook of combinatorial optimization, vol. 4. Springer, 1998. 
[39] J. W. Chinneck, Feasibility and Infeasibility in Optimization: Algorithms and Computational Methods, vol. 118. Springer Science \& Business Media, 2007.

[40] J. Meller, M. Wagner, and R. Elber, "Maximum feasibility guideline in the design and analysis of protein folding potentials," Journal of Computational Chemistry, vol. 23, no. 1, pp. 111-118, 2002.

[41] J. W. Chinneck, "Integrated classifier hyperplane placement and feature selection," Expert Systems with Applications, vol. 39, no. 9, pp. 8193-8203, 2012.

[42] A. P. D. Silva, "Optimization approaches to supervised classification," European Journal of Operational Research, vol. 261, no. 2, pp. 772-788, 2017.

[43] M. Rani, S. Dhok, and R. Deshmukh, "A systematic review of compressive sensing: Concepts, implementations and applications," IEEE Access, vol. 6, pp. 4875-4894, 2018.

[44] Y. Arjoune, N. Kaabouch, H. El Ghazi, and A. Tamtaoui, "Compressive sensing: Performance comparison of sparse recovery algorithms," in 2017 IEEE 7th Annual Computing and Communication Workshop and Conference $(C C W C)$, pp. 1-7, 2017.

[45] S. Peter, Algorithms for Robust and Fast Sparse Recovery. PhD thesis, Technische Universität München, 2016.

[46] R. Killick, P. Fearnhead, and I. A. Eckley, "Optimal detection of changepoints with a linear computational cost," Journal of the American Statistical Association, vol. 107, no. 500, pp. 1590-1598, 2012.

[47] Mosek ApS, The MOSEK optimization toolbox for MATLAB manual. Version 9.2., 2021. 
[48] A. Patrizio, "Idc: Expect 175 zettabytes of data worldwide by 2025," Network World, 2018.

[49] "National cancer institute." [Online]. Available: https://www.cancer.gov/ research/areas/diagnosis/artificial-intelligence.

[50] "Roundup of machine learning forecasts and market estimates, 2020." [Online]. Available: https://www.forbes.com/sites/louiscolumbus/2020/01/19/ roundup-of-machine-learning-forecasts-and-market-estimates-2020/ ? sh=3c1047ec5c02.

[51] R. F. Thompson, G. Valdes, C. D. Fuller, C. M. Carpenter, O. Morin, S. Aneja, W. D. Lindsay, H. J. Aerts, B. Agrimson, C. Deville Jr, et al., "Artificial intelligence in radiation oncology: a specialty-wide disruptive transformation?," Radiotherapy and Oncology, vol. 129, no. 3, pp. 421-426, 2018.

[52] Q. Liu, L. Yu, L. Luo, Q. Dou, and P. A. Heng, "Semi-supervised medical image classification with relation-driven self-ensembling model," IEEE Transactions on Medical Imaging, vol. 39, no. 11, pp. 3429-3440, 2020.

[53] A. P. Jain and P. Dandannavar, "Application of machine learning techniques to sentiment analysis," in 2016 2nd International Conference on Applied and Theoretical Computing and Communication Technology (iCATccT), pp. 628632.

[54] M. Leo, S. Sharma, and K. Maddulety, "Machine learning in banking risk management: A literature review," Risks, vol. 7, no. 1, p. 29, 2019. 
[55] A. Singh, N. Thakur, and A. Sharma, "A review of supervised machine learning algorithms," in 3rd International Conference on Computing for Sustainable Global Development (INDIACom), pp. 1310-1315.

[56] V. Bahel, S. Pillai, and M. Malhotra, "A comparative study on various binary classification algorithms and their improved variant for optimal performance," in 2020 IEEE Region 10 Symposium (TENSYMP), pp. 495-498.

[57] L. E. Peterson, "K-nearest neighbor," Scholarpedia, vol. 4, no. 2, p. 1883, 2009.

[58] S. Tong and D. Koller, "Support vector machine active learning with applications to text classification," Journal of Machine Learning Research, vol. 2, no. Nov, pp. 45-66, 2001.

[59] I. Rish et al., "An empirical study of the naive bayes classifier," in IJCAI 2001 Workshop on Empirical Methods in Artificial Intelligence, vol. 3, pp. 41-46.

[60] A. I. Schein and L. H. Ungar, "Active learning for logistic regression: an evaluation," Machine Learning, vol. 68, no. 3, pp. 235-265, 2007.

[61] N. Bhatia and Vandana, "Survey of nearest neighbor techniques," International Journal of Computer Science and Information Security, vol. 8, pp. 302-305, 2010.

[62] S. Archana and K. Elangovan, "Survey of classification techniques in data mining," International Journal of Computer Science and Mobile Applications, vol. 2, no. 2, pp. 65-71, 2014.

[63] K. P. Murphy, "Naive bayes classifiers," University of British Columbia, vol. 18, p. $60,2006$. 
[64] C. Bustamante, L. Garrido, and R. Soto, "Comparing fuzzy naive bayes and gaussian naive bayes for decision making in robocup 3d," in 2006 Mexican International Conference on Artificial Intelligence, pp. 237-247.

[65] D. Dua and C. Graff, "UCI machine learning repository." [Online]. Available: http://archive.ics.uci.edu/ml.

[66] J. Cervantes, F. Garcia-Lamont, L. Rodríguez-Mazahua, and A. Lopez, "A comprehensive survey on support vector machine classification: Applications, challenges and trends," Neurocomputing, vol. 408, pp. 189-215, 2020.

[67] L. Yang and A. Shami, "On hyperparameter optimization of machine learning algorithms: Theory and practice," Neurocomputing, vol. 415, pp. 295-316, 2020.

[68] L. Li, K. Jamieson, G. De Salvo, R. A. Talwalkar, and A. Hyperband, "A novel bandit-based approach to hyperparameter optimization," Computer Vision and Pattern Recognition, arXiv: 1603.0656, 2016.

[69] K. Eggensperger, F. Hutter, H. Hoos, and K. Leyton-Brown, "Efficient benchmarking of hyperparameter optimizers via surrogates," in AAAI Conference on Artificial Intelligence, vol. 29, 2015.

[70] F. Pedregosa, G. Varoquaux, A. Gramfort, V. Michel, B. Thirion, O. Grisel, M. Blondel, P. Prettenhofer, R. Weiss, V. Dubourg, J. Vanderplas, A. Passos, D. Cournapeau, M. Brucher, M. Perrot, and E. Duchesnay, "Scikit-learn: Machine learning in Python," Journal of Machine Learning Research, vol. 12, pp. 2825-2830, 2011.

[71] R. G. Baraniuk, "Compressive sensing," IEEE Signal Processing Magazine, vol. 24, no. 4, pp. 118-121, 2007. 
[72] E. Candès, J. Romberg, and T. Tao, "Robust uncertainty principles: Exact signal reconstruction from highly incomplete frequency information," IEEE Transactions on Information Theory, vol. 52, no. 2, p. 489-509, 2006.

[73] D. Donoho, "Compressed sensing," IEEE Transactions on Information Theory, vol. 52, no. 4, pp. 1289-1306, 2006.

[74] E. Candès, "Compressive sampling," International Congress of Mathematicians, vol. 3, pp. 1433-1452, 2006.

[75] E. J. Candes and T. Tao, "Near-optimal signal recovery from random projections: Universal encoding strategies?," IEEE Transactions on Information Theory, vol. 52, no. 12, pp. 5406-5425, 2006.

[76] E. Candès and T. Tao, "Decoding by linear programming," IEEE Transactions on Information Theory, vol. 51, no. 12, pp. 4203 - 4215, 2005.

[77] D. L. Donoho and X. Huo, "Uncertainty principles and ideal atomic decomposition," IEEE Transactions on Information Theory, vol. 47, no. 7, pp. 2845 2862, 2001.

[78] E. J. Candès and M. B. Wakin, "An introduction to compressive sampling," IEEE Signal Processing Magazine, vol. 25, no. 2, pp. 21-30, 2008.

[79] Arjoune, Youness, Kaabouch, Naima, E. Ghazi, Hassan, Tamtaoui, and Ahmed, "A performance comparison of measurement matrices in compressive sensing," International Journal of Communication Systems, vol. 31, no. 10, pp. 1-9, 2018.

[80] R. Baraniuk, M. Davenport, R. DeVore, and M. Wakin, "A simple proof of the restricted isometry property for random matrices," Constructive Approximation, vol. 28, no. 3, pp. 253-263, 2008. 
[81] E. J. Candès, "The restricted isometry property and its implications for compressed sensing," Comptes Rendus Mathematique, vol. 346, no. 9-10, pp. 589$592,2008$.

[82] H. Rauhut, "Compressive sensing and structured random matrices," Theoretical Foundations and Numerical Methods for Sparse Recovery, vol. 9, pp. 1-92, 2010.

[83] H. L. Yap, M. B. Wakin, and C. J. Rozell, "Stable manifold embeddings with structured random matrices," IEEE Journal of Selected Topics in Signal Processing, vol. 7, no. 4, pp. 720-730, 2013.

[84] W. U. Bajwa, J. D. Haupt, G. M. Raz, S. J. Wright, and R. D. Nowak, "Toeplitzstructured compressed sensing matrices," in 2007 IEEE/SP 14th Workshop on Statistical Signal Processing, pp. 294-298.

[85] G. E. Pfander, H. Rauhut, and J. A. Tropp, "The restricted isometry property for time-frequency structured random matrices," Probability Theory and Related Fields, vol. 156, no. 3, pp. 707-737, 2013.

[86] H. Rauhut, "Compressive sensing and structured random matrices," Theoretical Foundations and Numerical Methods for Sparse Recovery, vol. 9, pp. 1-92, 2010.

[87] K. Życzkowski, K. A. Penson, I. Nechita, and B. Collins, "Generating random density matrices," Journal of Mathematical Physics, vol. 52, no. 6, p. 062201, 2011.

[88] J. Aljadeff, D. Renfrew, and M. Stern, "Eigenvalues of block structured asymmetric random matrices," Journal of Mathematical Physics, vol. 56, no. 10, p. $103502,2015$. 
[89] H. Rauhut, J. Romberg, and J. A. Tropp, "Restricted isometries for partial random circulant matrices," Applied and Computational Harmonic Analysis, vol. 32, no. 2, pp. $242-254,2012$.

[90] L. Yu, J. P. Barbot, G. Zheng, and H. Sun, "Toeplitz-structured chaotic sensing matrix for compressive sensing," in 2010 7th International Symposium on Communication Systems, Networks Digital Signal Processing (CSNDSP 2010), pp. 229-233, 2010.

[91] K. Li, L. Gan, and C. Ling, "Convolutional compressed sensing using deterministic sequences," IEEE Transactions on Signal Processing, vol. 61, no. 3, pp. 740-752, 2013.

[92] R. R. Naidu, P. Jampana, and C. S. Sastry, "Deterministic compressed sensing matrices: Construction via euler squares and applications," IEEE Transactions on Signal Processing, vol. 64, no. 14, pp. 3566-3575, 2016.

[93] L. Applebaum, S. D. Howard, S. Searle, and R. Calderbank, "Chirp sensing codes: Deterministic compressed sensing measurements for fast recovery," $\mathrm{Ap}$ plied and Computational Harmonic Analysis, vol. 26, no. 2, pp. 283-290, 2009.

[94] S. D. Howard, A. R. Calderbank, and S. J. Searle, "A fast reconstruction algorithm for deterministic compressive sensing using second order reed-muller codes," in 42nd Annual Conference on Information Sciences and Systems, pp. 11-15, 2008.

[95] N. Ailon and E. Liberty, "Fast dimension reduction using rademacher series on dual BCH codes," Discrete \& Computational Geometry, vol. 42, no. 4, p. 615, 2008. 
[96] R. Calderbank, S. Howard, and S. Jafarpour, "Construction of a large class of deterministic sensing matrices that satisfy a statistical isometry property," IEEE Journal of Selected Topics in Signal Processing, vol. 4, no. 2, pp. 358-374, 2010.

[97] A. Amini and F. Marvasti, "Deterministic construction of binary, bipolar, and ternary compressed sensing matrices," IEEE Transactions on Information Theory, vol. 57, no. 4, pp. 2360-2370, 2011.

[98] A. Amini, V. Montazerhodjat, and F. Marvasti, "Matrices with small coherence using p-ary block codes," IEEE Transactions on Signal Processing, vol. 60, no. 1, pp. 172-181, 2012.

[99] N. K. Natarajan, "Sparse approximate solutions to linear," SIAM Journal on Computing, vol. 24, no. 2, pp. 227 - 234, 1995.

[100] G. Satat, M. Tancik, and R. Raskar, "Lensless imaging with compressive ultrafast sensing," IEEE Transactions on Computational Imaging, vol. 3, no. 3, pp. 398-407, 2017.

[101] H. You, J. Zhu, and D. Parry, "Image reconstruction based on block-based compressive sensing," in 2015 Proceedings of the 38th Australasian Computer Science Conference (ACSC 2015), vol. 27, p. 30.

[102] M. F. Duarte, M. A. Davenport, D. Takhar, J. N. Laska, T. Sun, K. F. Kelly, and R. G. Baraniuk, "Single-pixel imaging via compressive sampling," IEEE Signal Processing Magazine, vol. 25, no. 2, pp. 83-91, 2008.

[103] F. Pareschi, P. Albertini, G. Frattini, M. Mangia, R. Rovatti, and G. Setti, "Hardware-algorithms co-design and implementation of an analog- 
to-information converter for biosignals based on compressed sensing," IEEE Transactions on Biomedical Circuits and Systems, vol. 10, no. 1, pp. 149-162, 2015 .

[104] S. Pudlewski, A. Prasanna, and T. Melodia, "Compressed-sensing-enabled video streaming for wireless multimedia sensor networks," IEEE Transactions on Mobile Computing, no. 6, pp. 1060-1072, 2012.

[105] R. G. Baraniuk, T. Goldstein, A. C. Sankaranarayanan, C. Studer, A. Veeraraghavan, and M. B. Wakin, "Compressive video sensing: algorithms, architectures, and applications," IEEE Signal Processing Magazine, vol. 34, no. 1, pp. 52-66, 2017.

[106] M. Herman and T. Strohmer, "Compressed sensing radar," in 2008 IEEE Radar Conference, pp. 1-6.

[107] M.-S. Kang, S.-J. Lee, S.-H. Lee, and K.-T. Kim, "Isar imaging of high-speed maneuvering target using gapped stepped-frequency waveform and compressive sensing," IEEE Transactions on Image Processing, vol. 26, no. 10, pp. 50435056, 2017.

[108] C. Karakus, A. C. Gurbuz, and B. Tavli, "Analysis of energy efficiency of compressive sensing in wireless sensor networks," IEEE Sensors Journal, vol. 13, no. 5, pp. 1999-2008, 2013.

[109] M. Hooshmand, M. Rossi, D. Zordan, and M. Zorzi, "Covariogram-based compressive sensing for environmental wireless sensor networks," IEEE Sensors Journal, vol. 16, no. 6, pp. 1716-1729, 2015. 
[110] Z. Li, H. Huang, and S. Misra, "Compressed sensing via dictionary learning and approximate message passing for multimedia internet of things," IEEE Internet of Things Journal, vol. 4, no. 2, pp. 505-512, 2016.

[111] M. Mangia, F. Pareschi, R. Rovatti, and G. Setti, "Low-cost security of iot sensor nodes with rakeness-based compressed sensing: Statistical and knownplaintext attacks," IEEE Transactions on Information Forensics and Security, vol. 13, no. 2, pp. 327-340, 2017.

[112] P. Jain and P. Kar, "Non-convex optimization for machine learning," arXiv preprint arXiv:1712.07897, 2017.

[113] B. Sun, H. Feng, K. Chen, and X. Zhu, "A deep learning framework of quantized compressed sensing for wireless neural recording," IEEE Access, vol. 4, pp. 5169-5178, 2016.

[114] J. Lu, N. Verma, and N. K. Jha, "Compressed signal processing on nyquistsampled signals," IEEE Transactions on Computers, vol. 65, no. 11, pp. 32933303, 2016.

[115] D. E. Bellasi, L. Bettini, C. Benkeser, T. Burger, Q. Huang, and C. Studer, "Vlsi design of a monolithic compressive-sensing wideband analog-to-information converter," IEEE Journal on Emerging and Selected Topics in Circuits and Systems, vol. 3, no. 4, pp. 552-565, 2013.

[116] M. Pelissier and C. Studer, "Non-uniform wavelet sampling for RF analog-toinformation conversion," IEEE Transactions on Circuits and Systems I: Regular Papers, vol. 65, no. 2, pp. 471-484, 2017. 
[117] X. Ma, F. Yang, S. Liu, J. Song, and Z. Han, "Design and optimization on training sequence for mmwave communications: A new approach for sparse channel estimation in massive mimo," IEEE Journal on Selected Areas in Communications, vol. 35, no. 7, pp. 1486-1497, 2017.

[118] A. Ali and W. Hamouda, "Advances on spectrum sensing for cognitive radio networks: Theory and applications," IEEE Communications Surveys Tutorials, vol. 19, no. 2, pp. 1277-1304, 2017.

[119] J. F. Gemmeke, H. Van Hamme, B. Cranen, and L. Boves, "Compressive sensing for missing data imputation in noise robust speech recognition," IEEE Journal of Selected Topics in Signal Processing, vol. 4, no. 2, pp. 272-287, 2010.

[120] F. F. Firouzeh, S. Ghorshi, and S. Salsabili, "Compressed sensing based speech enhancement," in 2014 8th International Conference on Signal Processing and Communication Systems (ICSPCS), pp. 1-6.

[121] A. Y. Carmi, L. Mihaylova, and S. J. Godsill, Compressed sensing $\& 5$ sparse filtering. Springer, 2014.

[122] E. C. Marques, N. Maciel, L. Naviner, H. Cai, and J. Yang, "A review of sparse recovery algorithms," IEEE Access, vol. 7, pp. 1300-1322, 2018.

[123] S. Ji, Y. Xue, and L. Carin, "Bayesian compressive sensing," IEEE Transactions on signal processing, vol. 56, no. 6, pp. 2346-2356, 2008.

[124] J. Tanner and K. Wei, "Normalized iterative hard thresholding for matrix completion," SIAM Journal on Scientific Computing, vol. 35, no. 5, pp. S104-S125, 2013. 
[125] J. D. Blanchard, J. Tanner, and K. Wei, "CGIHT: conjugate gradient iterative hard thresholding for compressed sensing and matrix completion," Information and Inference: A Journal of the IMA, vol. 4, no. 4, pp. 289-327, 2015.

[126] X. Zhu, L. Dai, W. Dai, Z. Wang, and M. Moonen, "Tracking a dynamic sparse channel via differential orthogonal matching pursuit," in MILCOM 2015 - 2015 IEEE Military Communications Conference, pp. 792-797, 2015.

[127] H. Huang and A. Makur, "Backtracking-based matching pursuit method for sparse signal reconstruction," IEEE Signal Processing Letters, vol. 18, no. 7, pp. 391-394, 2011.

[128] A. C. Gilbert, M. J. Strauss, J. A. Tropp, and R. Vershynin, "Algorithmic linear dimension reduction in the l_1 norm for sparse vectors," arXiv preprint cs/0608079, 2006.

[129] A. Beck and M. Teboulle, "A fast iterative shrinkage-thresholding algorithm with application to wavelet-based image deblurring," in 2009 IEEE International Conference on Acoustics, Speech and Signal Processing, pp. 693-696.

[130] N. B. Karahanoglu and H. Erdogan, "Compressed sensing signal recovery via forward-backward pursuit," Digital Signal Processing, vol. 23, no. 5, pp. 1539$1548,2013$.

[131] S. Foucart, "Hard thresholding pursuit: an algorithm for compressive sensing," SIAM Journal on Numerical Analysis, vol. 49, no. 6, pp. 2543-2563, 2011.

[132] A. C. Gilbert, M. J. Strauss, J. A. Tropp, and R. Vershynin, "One sketch for all: fast algorithms for compressed sensing," in 2007 39th Association for Computing Machinery symposium on Theory of computing, pp. 237-246. 
[133] J. K. Pant and S. Krishnan, "Two-pass p-regularized least-squares algorithm for compressive sensing," in 2017 IEEE International Symposium on Circuits and Systems (ISCAS), pp. 1-4.

[134] W. Li, Estimation and tracking of rapidly time-varying broadband acoustic communication channels. PhD thesis, Massachusetts Institute of Technology and Woods Hole Oceanographic Institution, 2006.

[135] G. Mileounis, B. Babadi, N. Kalouptsidis, and V. Tarokh, "An adaptive greedy algorithm with application to nonlinear communications," IEEE Transactions on Signal Processing, vol. 58, no. 6, pp. 2998-3007, 2010.

[136] S. J. Wright, R. D. Nowak, and M. A. Figueiredo, "Sparse reconstruction by separable approximation," IEEE Transactions on Signal Processing, vol. 57, no. 7, pp. 2479-2493, 2009.

[137] E. Vlachos, A. S. Lalos, and K. Berberidis, "Stochastic gradient pursuit for adaptive equalization of sparse multipath channels," IEEE Journal on Emerging and Selected Topics in Circuits and Systems, vol. 2, no. 3, pp. 413-423, 2012.

[138] B. A. Olshausen and K. J. Millman, "Learning sparse codes with a mixtureof-gaussians prior," in Advances in Neural Information Processing Systems, pp. $841-847,2000$.

[139] J. Lee, J. W. Choi, and B. Shim, "Sparse signal recovery via tree search matching pursuit," Journal of Communications and Networks, vol. 18, no. 5, pp. 699$712,2016$. 
[140] S. Rangan, P. Schniter, and A. K. Fletcher, "Vector approximate message passing," IEEE Transactions on Information Theory, vol. 65, no. 10, pp. 6664-6684, 2019 .

[141] C. R. Berger, Z. Wang, J. Huang, and S. Zhou, "Application of compressive sensing to sparse channel estimation," IEEE Communications Magazine, vol. 48, no. 11, pp. 164-174, 2010.

[142] S. S. Chen, "Basis pursuit," Ph.D. dissertation, Stanford Univ., Stanford, CA, 1995.

[143] S. S. Chen, D. L. Donoho, and M. A. Saunders, "Atomic decomposition by basis pursuit," SIAM J. Scientific Computing, vol. 20, no. 1, pp. 33 - 61, 1999.

[144] R. Tibshirani, "Regression shrinkage and selection via the lasso," Journal of the Royal Statistical Society: Series B (Methodological), vol. 58, no. 1, pp. 267-288, 1996.

[145] S. S. Chen, D. L. Donoho, and M. A. Saunders, "Atomic decomposition by basis pursuit," SIAM Journal on Computing, vol. 20, no. 1, pp. 33-61, 1998.

[146] B. Efron, T. Hastie, I. Johnstone, R. Tibshirani, et al., "Least angle regression," The Annals of Statistics, vol. 32, no. 2, pp. 407-499, 2004.

[147] I. Daubechies, M. Defrise, and C. De Mol, "An iterative thresholding algorithm for linear inverse problems with a sparsity constraint," Communications on Pure and Applied Mathematics: A Journal Issued by the Courant Institute of Mathematical Sciences, vol. 57, no. 11, pp. 1413-1457, 2004.

[148] P. S. Huggins and S. W. Zucker, "Greedy basis pursuit," IEEE Transactions on Signal Processing, vol. 55, no. 7, pp. 3760-3772, 2007. 
[149] E. Candès, T. Tao, et al., "The dantzig selector: Statistical estimation when $\mathrm{p}$ is much larger than n," The annals of Statistics, vol. 35, no. 6, pp. 2313-2351, 2007.

[150] B. Efron, T. Hastie, and R. Tibshirani, "Discussion: The dantzig selector: Statistical estimation when p is much larger than n," The Annals of Statistics, vol. 35 , no. 6, pp. 2358-2364, 2007.

[151] D. L. Donoho, A. Maleki, and A. Montanari, "Message-passing algorithms for compressed sensing," in 2009 National Academy of Sciences, vol. 106, no. 45, pp. 18914-18919.

[152] R. Garg and R. Khandekar, "Gradient descent with sparsification: an iterative algorithm for sparse recovery with restricted isometry property," in Proceedings of the 26th annual international conference on machine learning, pp. 337-344, 2009 .

[153] G. H. Mohimani, M. Babaie-Zadeh, and C. Jutten, "Fast sparse representation based on smoothed $\ell_{0}$ norm," in 2007 International Conference on Independent Component Analysis and Signal Separation, pp. 389-396.

[154] A. Moshtaghpour, L. Jacques, V. Cambareri, K. Degraux, and C. De Vleeschouwer, "Consistent basis pursuit for signal and matrix estimates in quantized compressed sensing," IEEE Signal Processing Letters, vol. 23, no. 1, pp. 25-29, 2015.

[155] H. Zanddizari, S. Rajan, and H. Zarrabi, "Increasing the quality of reconstructed signal in compressive sensing utilizing kronecker technique," Biomedical Engineering Letters, vol. 8, no. 2, pp. 239-247, 2018. 
[156] D. Mitra, H. Zanddizari, and S. Rajan, "Investigation of kronecker-based recovery of compressed ecg signal," IEEE Transactions on Instrumentation and Measurement, vol. 69, no. 6, pp. 3642-3653, 2019.

[157] W. Dai and O. Milenkovic, "Subspace pursuit for compressive sensing signal reconstruction," IEEE Transactions on Information Theory, vol. 55, no. 5, pp. 2230-2249, 2009.

[158] S. Qaisar, R. M. Bilal, W. Iqbal, M. Naureen, and S. Lee, "Compressive sensing: From theory to applications, a survey," Journal of Communications and networks, vol. 15, no. 5, pp. 443-456, 2013.

[159] G. H. Mohimani, M. Babaie-Zadeh, and C. Jutten, "Fast sparse representation based on smoothed 0 norm," in International Conference on Independent Component Analysis and Signal Separation, pp. 389-396, Springer, 2007.

[160] R. Chartrand, "Exact reconstruction of sparse signals via nonconvex minimization," IEEE Signal Processing Letters, vol. 14, no. 10, pp. 707-710, 2007.

[161] D. Kanevsky, A. Carmi, L. Horesh, P. Gurfil, B. Ramabhadran, and T. N. Sainath, "Kalman filtering for compressed sensing," in 201013 th International Conference on Information Fusion, pp. 1-8.

[162] I. F. Gorodnitsky and B. D. Rao, "Sparse signal reconstruction from limited data using focuss: A re-weighted minimum norm algorithm," IEEE Transactions on Signal Processing, vol. 45, no. 3, pp. 600-616, 1997.

[163] B. D. Rao and K. Kreutz-Delgado, "An affine scaling methodology for best basis selection," IEEE Transactions on Signal Processing, vol. 47, no. 1, pp. 187-200, 1999. 
[164] R. Chartrand and W. Yin, "Iteratively reweighted algorithms for compressive sensing," in 2008 IEEE International Conference on Acoustics, Speech and Signal Processing, pp. 3869-3872.

[165] R. Baraniuk, M. A. Davenport, M. F. Duarte, C. Hegde, et al., "An introduction to compressive sensing," Connexions e-textbook, pp. 24-76, 2011.

[166] S. G. Mallat and Z. Zhang, "Matching pursuits with time-frequency dictionaries," IEEE Transactions on Signal Processing, vol. 41, no. 12, pp. 3397-3415, 1993.

[167] Y. C. Pati, R. Rezaiifar, and P. S. Krishnaprasad, "Orthogonal matching pursuit: Recursive function approximation with applications to wavelet decomposition," in 1993 Proceedings of 27th Asilomar conference on signals, systems and computers, pp. 40-44.

[168] M. D. Plumbley, "Recovery of sparse representations by polytope faces pursuit," in 2006 International Conference on Independent Component Analysis and Signal Separation, pp. 206-213.

[169] T. Blumensath and M. E. Davies, "Gradient pursuits," IEEE Transactions on Signal Processing, vol. 56, no. 6, pp. 2370-2382, 2008.

[170] D. Needell and J. A. Tropp, "Cosamp: Iterative signal recovery from incomplete and inaccurate samples," Applied and Computational Harmonic Analysis, vol. 26, no. 3, pp. 301-321, 2009.

[171] T. Blumensath and M. E. Davies, "Iterative hard thresholding for compressed sensing," Applied and Computational Harmonic Analysis, vol. 27, no. 3, pp. 265-274, 2009. 
[172] D. Needell and R. Vershynin, "Uniform uncertainty principle and signal recovery via regularized orthogonal matching pursuit," Foundations of Computational Mathematics, vol. 9, no. 3, pp. 317-334, 2009.

[173] D. L. Donoho, Y. Tsaig, I. Drori, and J.-L. Starck, "Sparse solution of underdetermined systems of linear equations by stagewise orthogonal matching pursuit," IEEE Transactions on Information Theory, vol. 58, no. 2, pp. 10941121, 2012.

[174] J. Wang, S. Kwon, and B. Shim, "Generalized orthogonal matching pursuit," IEEE Transactions on Signal Processing, vol. 60, no. 12, pp. 6202-6216, 2012.

[175] H. Sun and L. Ni, "Compressed sensing data reconstruction using adaptive generalized orthogonal matching pursuit algorithm," in Proceedings of 2013 3rd International Conference on Computer Science and Network Technology, pp. 1102-1106, 2013.

[176] S. Kwon, J. Wang, and B. Shim, "Multipath matching pursuit," IEEE Transactions on Information Theory, vol. 60, no. 5, pp. 2986-3001, 2014.

[177] S. Narayanan, S. K. Sahoo, and A. Makur, "Greedy pursuits assisted basis pursuit for reconstruction of joint-sparse signals," Signal Processing, vol. 142, pp. 485-491, 2018.

[178] S. Mallat and Z. Zhang, "Matching pursuit with time-frequency dictionaries," IEEE Transactions on Signal Processing, vol. 41, no. 12, pp. 3397 - 3415, 1993.

[179] A. K. Mishra and R. S. Verster, Compressive sensing based algorithms for electronic defence. Springer, 2017. 
[180] J. A. Tropp and A. C. Gilbert, "Signal recovery from random measurements via orthogonal matching pursuit," IEEE Transactions on Information Theory, vol. 53, no. 12, pp. 4655-4666, 2007.

[181] F. Salahdine, N. Kaabouch, and H. El Ghazi, "A survey on compressive sensing techniques for cognitive radio networks," Physical Communication, vol. 20, pp. 61-73, 2016.

[182] S. B. Meenakshi, "A survey of compressive sensing based greedy pursuit reconstruction algorithms," International Journal of Image, Graphics and Signal Processing, vol. 7, no. 10, pp. 1-10, 2015.

[183] M. A. Hameed, Comparative analysis of orthogonal matching pursuit and least angle regression. Michigan State University, Electrical Engineering, 2012.

[184] H. Mohimani, M. Babaie-Zadeh, and C. Jutten, "A fast approach for overcomplete sparse decomposition based on smoothed $\ell_{0}$ norm," IEEE Transactions on Signal Processing, vol. 57, no. 1, pp. 289-301, 2008.

[185] "World health statistics 2019." [Online]. Available: http://apps.who.int/ iris/bitstream/handle/10665/311696/WHO-DAD-2019.1-eng.pdf.

[186] "Heart disease and stroke statistics-2019 at-a-glance." [Online]. Available: https://professional.heart.org/en/science-news/-/media/ 22cf5db5b1a24b38a435fcecb42d588b.ashx, 2019.

[187] N. Ji, T. Xiang, P. Bonato, N. H. Lovell, S.-Y. Ooi, D. A. Clifton, M. Akay, X.-R. Ding, B. P. Yan, V. Mok, D. I. Fotiadis, and Y.-T. Zhang, "Recommendation to use wearable-based mhealth in closed-loop management of acute cardiovascular 
disease patients during the COVID-19 pandemic," IEEE Journal of Biomedical and Health Informatics, vol. 25, no. 4, pp. 903-908, 2021.

[188] K. J. Clerkin, J. A. Fried, J. Raikhelkar, G. Sayer, J. M. Griffin, A. Masoumi, S. S. Jain, D. Burkhoff, D. Kumaraiah, L. Rabbani, et al., "COVID-19 and cardiovascular disease," Circulation, vol. 141, no. 20, pp. 1648-1655, 2020.

[189] D. M. Roden, R. A. Harrington, A. Poppas, and A. M. Russo, "Considerations for drug interactions on qtc in exploratory COVID-19 treatment," Circulation, vol. 141, no. 24, pp. e906-e907, 2020.

[190] B. S. Kim, S. K. Yoo, and M.-H. Lee, "Wavelet-based low-delay ecg compression algorithm for continuous ecg transmission," IEEE Transactions on Information Technology in Biomedicine, vol. 10, no. 1, pp. 77-83, 2006.

[191] J. P. Abenstein and W. J. Tompkins, "A new data-reduction algorithm for real-time ecg analysis," IEEE Transactions on Biomedical Engineering, no. 1, pp. 43-48, 1982.

[192] J. Cox, F. Nolle, H. Fozzard, and G. Oliver, "Aztec, a preprocessing program for real-time ecg rhythm analysis," IEEE Transactions on Biomedical Engineering, no. 2, pp. 128-129, 1968.

[193] M. Abdelazez, S. Rajan, and A. D. Chan, "Impact of motion artifact on detection of atrial fibrillation in compressively sensed ECG using a deterministic matrix," 2019 CMBES Proceedings, vol. 42.

[194] "MIT-BIH arrhythmia database." [Online]. Available: http://www. physionet.org/physiobank/database/mitdb. 
[195] Y. Zigel, A. Cohen, and A. Katz, "The weighted diagnostic distortion (WDD) measure for ECG signal compression," IEEE Transactions on Biomedical Engineering, vol. 47, no. 11, pp. 1422-1430, 2000.

[196] G. B. Moody and R. G. Mark, "The impact of the MIT-BIH Arrhythmia Database," IEEE Engineering in Medicine and Biology Magazine, vol. 20, no. 3, pp. 45-50, 2001.

[197] A. Němcová, R. Smíšek, L. Maršánová, L. Smital, and M. Vítek, "A comparative analysis of methods for evaluation of ecg signal quality after compression," BioMed Research International, vol. 9, 2018.

[198] J. Hrubeš, M. Vitek, and J. Kozumplik, "Multipoint validation of decompressed ecg signal," Analysis of Biomedical Signals and Images, vol. 20, no. 1, 2010.

[199] S. Chatterjee, D. Sundman, and M. Skoglund, "Look ahead orthogonal matching pursuit," in 2011 IEEE International Conference on Acoustics, Speech and Signal Processing (ICASSP), pp. 4024-4027.

[200] D. L. Donoho, V. C. Stodden, and Y. Tsaig, "About sparselab." [Online]. Available: https://sparselab.stanford.edu/, 2007.

[201] C. Chen, J. Huang, L. He, and H. Li, "Preconditioning for accelerated iteratively reweighted least squares in structured sparsity reconstruction," in 2014 IEEE Conference on Computer Vision and Pattern Recognition, pp. 2713-2720.

[202] C. Chen, J. Huang, L. He, and H. Li, "Fast iteratively reweighted least squares algorithms for analysis-based sparsity reconstruction," arXiv preprint arXiv:1411.5057, 2014. 
[203] S. Perez, "Report: Voice assistants in use to triple to 8 billion by 2023." [Online]. Available: http://social.techcrunch.com/2019/02/12/ report-voice-assistants-in-use-to-triple-to-8-billion-by-2023/.

[204] K. Sayood, Introduction to data compression. Morgan Kaufmann, 2017.

[205] F. A. Muin, T. S. Gunawan, M. Kartiwi, and E. M. A. Elsheikh, "A review of lossless audio compression standards and algorithms," AIP Conference Proceedings, vol. 1883, no. 1, p. 020006, 2017.

[206] K. Paliwal and K. Wójcicki, "Effect of analysis window duration on speech intelligibility," IEEE EEE Signal Processing Letters, vol. 15, pp. 785-788, 2008.

[207] E. J. Candès and M. B. Wakin, "An introduction to compressive sampling [a sensing/sampling paradigm that goes against the common knowledge in data acquisition]," IEEE Signal Processing Magazine, vol. 25, no. 2, pp. 21-30, 2008.

[208] V. Abrol, P. Sharma, and S. Budhiraja, "Evaluating performance of compressed sensing for speech signals," in 2013 3rd IEEE International Advance Computing Conference (IACC), pp. 1159-1164.

[209] U. P. Shukla, N. B. Patel, and A. M. Joshi, "A survey on recent advances in speech compressive sensing," in International Mutli-Conference on Automation, Computing, Communication, Control and Compressed Sensing (iMac4s), pp. 276-280, 2013.

[210] T. V. Sreenivas and W. B. Kleijn, "Compressive sensing for sparsely excited speech signals," in 2009 IEEE International Conference on Acoustics, Speech and Signal Processing, pp. 4125-4128. 
[211] J. Garofolo, L. Lamel, W. Fisher, J. Fiscus, D. Pallett, N. Dahlgren, and V. Zue, "Timit acoustic-phonetic continuous speech corpus." [Online]. Available: https://hdl.handle.net/11272.1/AB2/SWVENO, 1993.

[212] A. W. Rix, J. G. Beerends, M. P. Hollier, and A. P. Hekstra, "Perceptual evaluation of speech quality (PESQ)-a new method for speech quality assessment of telephone networks and codecs," in 2001 IEEE International Conference on Acoustics, Speech, and Signal Processing. Proceedings, vol. 2, pp. 749-752.

[213] I.-T. Recommendation, "Perceptual evaluation of speech quality (pesq): An objective method for end-to-end speech quality assessment of narrow-band telephone networks and speech codecs," Rec. ITU-T P. 862, 2001.

[214] G. H. Golub and C. Reinsch, "Singular value decomposition and least squares solutions," in Linear Algebra, pp. 134-151, Springer, 1971.

[215] S. Wold, K. Esbensen, and P. Geladi, "Principal component analysis," Chemometrics and Intelligent Laboratory Systems, vol. 2, no. 1-3, pp. 37-52, 1987.

[216] K. Fukunaga, Introduction to statistical pattern recognition. Elsevier, 2013.

[217] P. Comon, "Independent component analysis, a new concept?," Signal Processing, vol. 36, no. 3, pp. 287-314, 1994.

[218] R. Gray, "Vector quantization," IEEE Assp Magazine, vol. 1, no. 2, pp. 4-29, 1984.

[219] P. Paatero and U. Tapper, "Positive matrix factorization: A non-negative factor model with optimal utilization of error estimates of data values," Environmetrics, vol. 5, no. 2, pp. 111-126, 1994. 
[220] D. D. Lee and H. S. Seung, "Learning the parts of objects by non-negative matrix factorization," Nature, vol. 401, no. 6755, pp. 788-791, 1999.

[221] S. A. Vavasis, "On the complexity of nonnegative matrix factorization," SIAM Journal on Optimization, vol. 20, no. 3, pp. 1364 - 1377, 2008.

[222] D. D. Lee and H. S. Seung, "Algorithms for non-negative matrix factorization," in Advances in Neural Information Processing Systems, pp. 556-562, 2001.

[223] T.-H. Chan, W.-K. Ma, A. Ambikapathi, and C.-Y. Chi, "A simplex volume maximization framework for hyperspectral endmember extraction," IEEE Transactions on Geoscience and Remote Sensing, vol. 49, no. 11, pp. 4177-4193, 2011.

[224] R. Zdunek, "Initialization of nonnegative matrix factorization with vertices of convex polytope," in 2012 International Conference on Artificial Intelligence and Soft Computing, pp. 448-455.

[225] D. Donoho and V. Stodden, "When does non-negative matrix factorization give a correct decomposition into parts?," in Advances in neural information processing systems, pp. 1141-1148, 2004.

[226] N. Gillis, "Introduction to nonnegative matrix factorization," arXiv preprint arXiv:1703.00663, 2017.

[227] N. B. Erichson, A. Mendible, S. Wihlborn, and J. N. Kutz, "Randomized nonnegative matrix factorization," Pattern Recognition Letters, vol. 104, pp. 1-7, 2018. 
[228] J. Pan and N. Gillis, "Generalized separable nonnegative matrix factorization," IEEE Transactions on Pattern Analysis and Machine Intelligence, vol. 43, no. 5, pp. 1546-1561, 2021.

[229] N. Gillis, "Successive nonnegative projection algorithm for robust nonnegative blind source separation," SIAM Journal on Imaging Sciences, vol. 7, no. 2, pp. 1420-1450, 2014.

[230] A. Kumar, V. Sindhwani, and P. Kambadur, "Fast conical hull algorithms for near-separable non-negative matrix factorization," in 2013 International Conference on Machine Learning, pp. 231-239.

[231] N. Gillis and R. Luce, "A fast gradient method for nonnegative sparse regression with self-dictionary," IEEE Transactions on Image Processing, vol. 27, no. 1, pp. 24-37, 2017.

[232] N. Gillis and F. Glineur, "Accelerated multiplicative updates and hierarchical als algorithms for nonnegative matrix factorization," Neural computation, vol. 24, no. 4, pp. 1085-1105, 2012.

[233] X. Fu, K. Huang, B. Yang, W.-K. Ma, and N. D. Sidiropoulos, "Robust volume minimization-based matrix factorization for remote sensing and document clustering," IEEE Transactions on Signal Processing, vol. 64, no. 23, pp. 6254-6268, 2016 .

[234] M. C. U. Araújo, T. C. B. Saldanha, R. K. H. Galvao, T. Yoneyama, H. C. Chame, and V. Visani, "The successive projections algorithm for variable selection in spectroscopic multicomponent analysis," Chemometrics and Intelligent Laboratory Systems, vol. 57, no. 2, pp. 65-73, 2001. 
[235] N. Gillis, "The why and how of nonnegative matrix factorization," Regularization, Optimization, Kernels, and Support Vector Machines, vol. 12, no. 257, pp. 257-291, 2014.

[236] N. Gillis and S. A. Vavasis, "Fast and robust recursive algorithms for separable nonnegative matrix factorization," IEEE Transactions on Pattern Analysis and Machine Intelligence, vol. 36, no. 4, pp. 698-714, 2013.

[237] W.-K. Ma, J. M. Bioucas-Dias, T.-H. Chan, N. Gillis, P. Gader, A. J. Plaza, A. Ambikapathi, and C.-Y. Chi, "A signal processing perspective on hyperspectral unmixing: Insights from remote sensing," IEEE Signal Processing Magazine, vol. 31, no. 1, pp. 67-81, 2013.

[238] N. Gillis, "Successive projection algorithm robust to outliers," arXiv preprint arXiv:1908.04109, 2019.

[239] V. Leplat, A. M. Ang, and N. Gillis, "Minimum-volume rank-deficient nonnegative matrix factorizations," in ICASSP 2019-2019 IEEE International Conference on Acoustics, Speech and Signal Processing (ICASSP), pp. 3402-3406, 2019.

[240] L. Miao and H. Qi, "Endmember extraction from highly mixed data using minimum volume constrained nonnegative matrix factorization," IEEE Transactions on Geoscience and Remote Sensing, vol. 45, no. 3, pp. 765-777, 2007.

[241] L. Muzzarelli, S. Weis, S. B. Eickhoff, and K. R. Patil, "Rank selection in nonnegative matrix factorization: systematic comparison and a new mad metric," in 2019 International Joint Conference on Neural Networks (IJCNN), pp. 1-8, 2019. 
[242] I. Buciu, "Non-negative matrix factorization, a new tool for feature extraction: theory and applications," International Journal of Computers, Communications and Control, vol. 3, no. 3, pp. 67-74, 2008.

[243] L. Du, X. Li, and Y.-D. Shen, "Robust nonnegative matrix factorization via half-quadratic minimization," in 2012 IEEE 12th International Conference on Data Mining, pp. 201-210, 2012.

[244] S. Peng, W. Ser, B. Chen, L. Sun, and Z. Lin, "Robust nonnegative matrix factorization with local coordinate constraint for image clustering," Engineering Applications of Artificial Intelligence, vol. 88, p. 103354, 2020.

[245] P. Vaidya, "Speeding-up linear programming using fast matrix multiplication," in 30th Annual Symposium on Foundations of Computer Science, pp. 332-337, 1989.

[246] A. M. Syed, S. Qazi, and N. Gillis, "Improved svd-based initialization for nonnegative matrix factorization using low-rank correction," arXiv preprint arXiv:180\%.04020, 2018. 